\title{
One-Dimensional ZnO Nanostructures: Solution Growth and Functional Properties
}

\author{
Sheng Xu and Zhong Lin Wang ( $\varangle)$ \\ School of Materials Science and Engineering, Georgia Institute of Technology, Atlanta, Georgia 30332-0245, USA \\ Received: 8 May 2011 / Revised: 14 June 2011 / Accepted: 15 June 2011 \\ (C) Tsinghua University Press and Springer-Verlag Berlin Heidelberg 2011
}

\begin{abstract}
One-dimensional (1D) ZnO nanostructures have been studied intensively and extensively over the last decade not only for their remarkable chemical and physical properties, but also for their current and future diverse technological applications. This article gives a comprehensive overview of the progress that has been made within the context of $1 \mathrm{D} \mathrm{ZnO}$ nanostructures synthesized via wet chemical methods. We will cover the synthetic methodologies and corresponding growth mechanisms, different structures, doping and alloying, positioncontrolled growth on substrates, and finally, their functional properties as catalysts, hydrophobic surfaces, sensors, and in nanoelectronic, optical, optoelectronic, and energy harvesting devices.
\end{abstract}

\section{KEYWORDS}

$\mathrm{ZnO}$, one dimensional nanostructures, solution growth, semiconductive, optical, piezoelectric, novel devices

\section{Introduction}

$\mathrm{ZnO}$ is a semiconducting and piezoelectric material with a direct wide band gap of $3.37 \mathrm{eV}$ and a large exciton binding energy of $60 \mathrm{meV}$ at room temperature $[1,2]$. It has been demonstrated to have enormous applications in electronic, optoelectronic, electrochemical, and electromechanical devices [3-8], such as ultraviolet (UV) lasers [9, 10], light-emitting diodes [11], field emission devices [12-14], high performance nanosensors [15-17], solar cells [18-21], piezoelectric nanogenerators [22-24], and nanopiezotronics [25-27]. One-dimensional (1D) $\mathrm{ZnO}$ nanostructures have been synthesized by a wide range of techniques, such as wet chemical methods [28-30], physical vapor deposition [31-33], metal-organic chemical vapor deposition (MOCVD) [34-36], molecular beam epitaxy (MBE) [37], pulsed laser deposition [38, 39], sputtering [40], flux methods [41], eletrospinning [42-44], and even top-down approaches by etching [45]. Among those techniques, physical vapor deposition and flux methods usually require high temperature, and easily incorporate catalysts or impurities into the $\mathrm{ZnO}$ nanostructures. Therefore, they are less likely to be able to integrate with flexible organic substrates for future foldable and portable electronics. MOCVD and MBE can give high quality $\mathrm{ZnO}$ nanowire arrays, but are usually limited by the poor sample uniformity, low product yield, and choices of substrate. Also, the experimental cost is usually very high, so they have been less widely adopted. Pulsed laser deposition, sputtering and top down approaches have less controllability and repeatability compared with other techniques. Electrospinning gives polycrystalline fibers. Comparatively speaking, wet chemical methods are attractive for several reasons: they are low cost, less hazardous, and thus capable of

Address correspondence to zhong.wang@mse.gatech.edu 
easy scaling up [46, 47]; growth occurs at a relatively low temperature, compatible with flexible organic substrates; there is no need for the use of metal catalysts, and thus it can be integrated with well-developed silicon technologies [48]; in addition, there are a variety of parameters that can tuned to effectively control the morphologies and properties of the final products [49, 50]. Wet chemical methods have been demonstrated as a very powerful and versatile technique for growing $1 \mathrm{D} \mathrm{ZnO}$ nanostructures.

Here in this review, we focus on the $1 \mathrm{D} \mathrm{ZnO}$ nanostructures that have been grown by wet chemical methods, although evaluation of $\mathrm{ZnO}$ nanostructures is provided in the vast Ref. $[1,5,6,51-53]$. We cover the following five main aspects. First, we will go over the basic synthetic methodologies and growth mechanisms that have been adopted in the literature. Second, we will display the various kinds of novel nanostructures of $\mathrm{ZnO}$ that have been achieved by wet chemical methods. Third, we will summarize ways to manipulate the conductivity of the $\mathrm{ZnO}$ nanostructures by doping, such as $n$-type, $p$-type, and transition metal doping, and the ways of engineering the $\mathrm{ZnO}$ band gap by alloying with other metal oxides. Fourth, we will show the various techniques that have been implemented to control the spatial distribution of $\mathrm{ZnO}$ nanostructures on a substrate, namely patterned growth. Finally we will illustrate the functional properties of $1 \mathrm{D} \mathrm{ZnO}$ nanostructures and the diverse innovative applications where $1 \mathrm{D} \mathrm{ZnO}$ nanostructures play an important role.

\section{Basic synthetic methodologies and growth mechanisms}

$\mathrm{ZnO}$ is an amphoteric oxide with an isoelectric point value of about 9.5 [54]. Generally speaking, $\mathrm{ZnO}$ is expected to crystallize by the hydrolysis of $\mathrm{Zn}$ salts in a basic solution that can be formed using strong or weak alkalis. $\mathrm{Zn}^{2+}$ is known to coordinate in tetrahedral complexes. Due to the $3 \mathrm{~d}^{10}$ electron configuration, it is colorless and has zero crystal field stabilization energy. Depending on the given $\mathrm{pH}$ and temperature [55], $\mathrm{Zn}^{2+}$ is able to exist in a series of intermediates, and $\mathrm{ZnO}$ can be formed by the dehydration of these intermediates.

Chemical reactions in aqueous systems are usually considered to be in a reversible equilibrium, and the driving force is the minimization of the free energy of the entire reaction system, which is the intrinsic nature of wet chemical methods [56]. Wurtzite structured $\mathrm{ZnO}$ grown along the $c$ axis has high energy polar surfaces such as $\pm(0001)$ surfaces with alternating $\mathrm{Zn}^{2+}$-terminated and $\mathrm{O}^{2-}$-terminated surfaces [28]. So when a $\mathrm{ZnO}$ nucleus is newly formed, owing to the high energy of the polar surfaces, the incoming precursor molecules tend to favorably adsorb on the polar surfaces. However, after adsorption of one layer of precursor molecules, the polar surface transforms into another polar surface with inverted polarity. For instance, a $\mathrm{Zn}^{2+}$-terminated surface changes into an $\mathrm{O}^{2-}$-terminated surface, or vice versa. Such a process is repeated over time, leading to a fast growth along the \pm [0001] directions, exposing the non-polar $\{1 \overline{1} 00\}$ and $\{2 \overline{1} \overline{1} 0\}$ surfaces to the solution. This is essentially how a 1D nanostructure is formed.

\subsection{Growth in general alkaline solutions}

An alkaline solution is essential for the formation of $\mathrm{ZnO}$ nanostructures because normally divalent metal ions do not hydrolyze in acidic environments [28, 57, 58]. The commonly used alkali compounds are $\mathrm{KOH}$ and $\mathrm{NaOH}$. Generally speaking, the solubility of $\mathrm{ZnO}$ in an alkali solution increases with the alkali concentration and temperature. Supersaturation allows a growth zone to be attained [58]. $\mathrm{KOH}$ is thought to be preferable to $\mathrm{NaOH}$, because $\mathrm{K}^{+}$has a larger ion radius and thus a lower probability of incorporation into the ZnO lattice [58, 59]. Furthermore, it has been suggested that $\mathrm{Na}^{+}$is attracted by the $\mathrm{OH}^{-}$around the nanocrystal and forms a virtual capping layer, thus, inhibiting the nanocrystal growth [60].

$$
\begin{aligned}
& \mathrm{Zn}^{2+}+2 \mathrm{OH}^{-} \longleftrightarrow \mathrm{Zn}(\mathrm{OH})_{2} \\
& \mathrm{Zn}(\mathrm{OH})_{2}+2 \mathrm{OH}^{-} \longleftrightarrow\left[\mathrm{Zn}(\mathrm{OH})_{4}\right]^{2-} \\
& {\left[\mathrm{Zn}(\mathrm{OH})_{4}\right]^{2-} \longleftrightarrow \mathrm{ZnO}_{2}^{2-}+2 \mathrm{H}_{2} \mathrm{O}} \\
& \mathrm{ZnO}_{2}{ }^{2-}+\mathrm{H}_{2} \mathrm{O} \longleftrightarrow \mathrm{ZnO}+2 \mathrm{OH}^{-} \\
& \mathrm{ZnO}+\mathrm{OH}^{-} \longleftrightarrow \mathrm{ZnOH}^{-}
\end{aligned}
$$

The main reactions involved in the growth are illustrated in the above equations [61, 62]. For the 
equation (2), the product is not necessarily $\mathrm{Zn}(\mathrm{OH})_{4}{ }^{2-}$, but could also be in the form of $\mathrm{Zn}(\mathrm{OH})^{+}, \mathrm{Zn}(\mathrm{OH})_{2}$, or $\mathrm{Zn}(\mathrm{OH})_{3}{ }^{-}$, depending on the parameters, such as the concentration of $\mathrm{Zn}^{2+}$ and the $\mathrm{pH}$ value, as shown in Fig. 1(a). And all of these intermediate forms are actually in equilibrium, with the major forms being different under different reaction conditions. The growth process could be described as follows [63]. At the very beginning, the $\mathrm{Zn}^{2+}$ and $\mathrm{OH}^{-}$ions coordinate

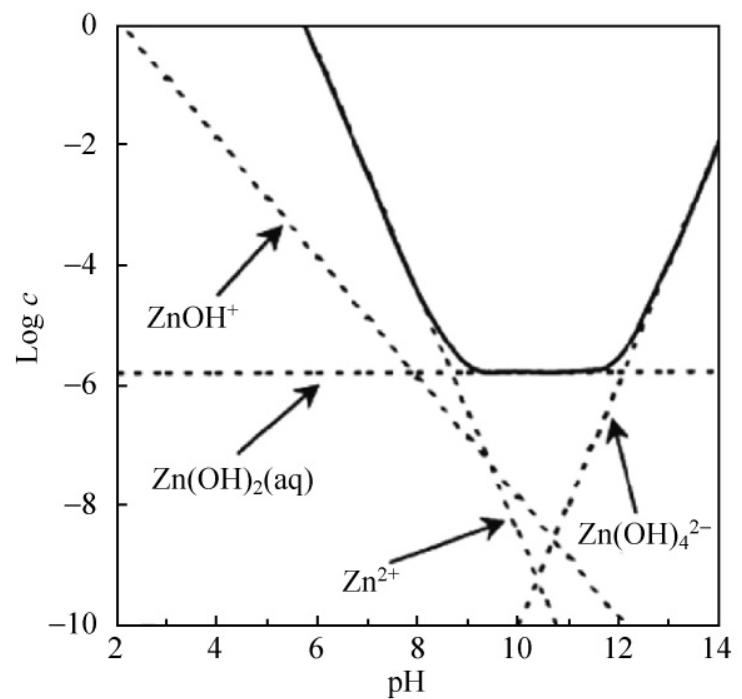

(a)

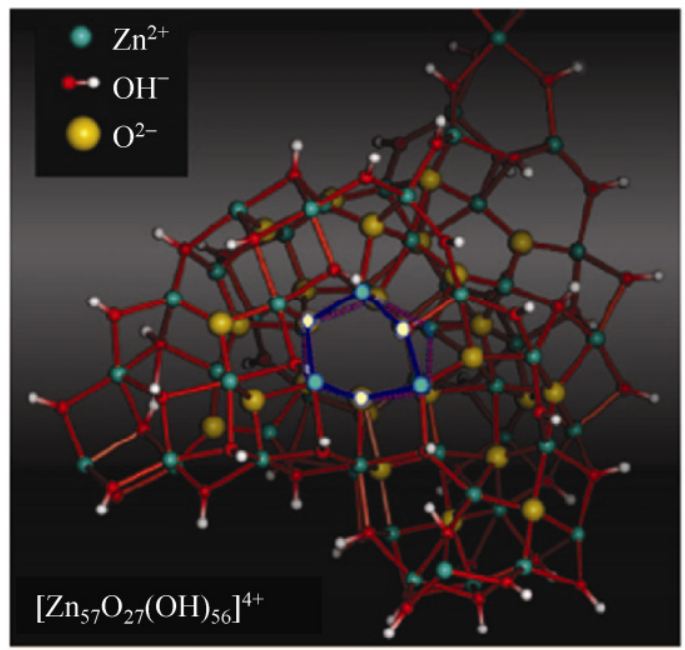

(b)

Figure 1 (a) Phase stability diagrams for the $\mathrm{ZnO}(\mathrm{s})-\mathrm{H}_{2} \mathrm{O}$ system at $25^{\circ} \mathrm{C}$ as a function of precursor concentration and $\mathrm{pH}$, where the dashed lines denote the thermodynamic equilibrium between the $\mathrm{Zn}^{2+}$ soluble species and the corresponding solid phases [64]. (b) Aggregation and nucleation of domains of the wurtzite structured $\mathrm{ZnO}$, where the characteristic six membered rings in the aggregate center are highlighted in blue. The two staggered six-rings form a center of stability and give rise to further ordering in favor of the wurtzite structure [63]. Reproduced with permission with each other, and then they undergo dehydration by proton transfer, forming $\mathrm{Zn}^{2+} \ldots \mathrm{O}^{2-\ldots} \mathrm{Zn}^{2+}$ bonds, and leading to an agglomerate of the form of $\left[\mathrm{Zn}_{x}(\mathrm{OH})_{y}\right]^{(2 x-y)+}$, which has an octahedral geometry. The $\mathrm{H}_{2} \mathrm{O}$ molecules formed by dehydration migrate into the solution. These aggregates usually contain fewer than 50 ions, and the formation of $\mathrm{O}^{2-}$ ions implies dramatic changes within the aggregate. After the aggregates reach around 150 ions, wurtzite type (tetrahedral coordination) $\mathrm{ZnO}$ domains are then nucleated in the central region of the aggregates (shown in Fig. 1(b)). The core comprises $\mathrm{Zn}^{2+}$ and $\mathrm{O}^{2-}$ ions only, while the aggregate surface still mainly consists of $\mathrm{Zn}^{2+}$ and $\mathrm{OH}^{-}$ions. Aggregates of over 200 ions exhibit a nanometer-sized core of the wurtzite structured $\mathrm{ZnO}$ which grows as a result of further association and dehydration of $\mathrm{Zn}^{2+}$ and $\mathrm{OH}^{-}$ ions [63].

In the above equations, the $\mathrm{O}^{2-}$ in $\mathrm{ZnO}$ comes from the base, not from the solvent $\mathrm{H}_{2} \mathrm{O}$. Therefore growth of $\mathrm{ZnO}$ does not necessarily require the solvent to be $\mathrm{H}_{2} \mathrm{O}$ [65]. It could be organic solvents, such as methanol [66], ethanol [67], and butanol [68], or even ionic liquids $[69,70]$. Under alkali conditions, the reactions could take place at room temperature by adjusting the ratio of $\mathrm{Zn}^{2+}$ and $\mathrm{OH}^{-}$, giving rise to $\mathrm{ZnO}$ nanowires with diameter even below $10 \mathrm{~nm}$. $\mathrm{ZnO}$ nanowires with various aspect ratios can be prepared by simply adjusting $\mathrm{OH}^{-}$concentration and reaction time [68].

The growth of polar inorganic nanocrystals is sensitive to the reaction solvents, and their morphologies could be tuned and controlled by the crystal-solvent interfacial interactions [66]. In such cases, the morphology of $\mathrm{ZnO}$ is largely directed by the polarity and saturated vapor pressure of the solvents [65]. As shown in Figs. 2(a)-2(c), the aspect ratio of $\mathrm{ZnO}$ nanowires, which is dictated by the relative growth rates of polar and nonpolar surfaces, can be readily tuned by varying the polarity of the solvents. Highly polar solvent molecules have stronger interactions with the polar surfaces of $\mathrm{ZnO}$, and thus hinder the precursor molecules from adsorbing and settling down onto the polar surfaces. The aspect ratio of the $\mathrm{ZnO}$ nanostructures increases on going from the more polar solvent methanol to the less polar solvent 1-butanol. All the as-grown $\mathrm{ZnO}$ nanowires showed two wellfaceted basal planes along the $\pm c$ axis as shown in Fig. 2(d) [67]. 

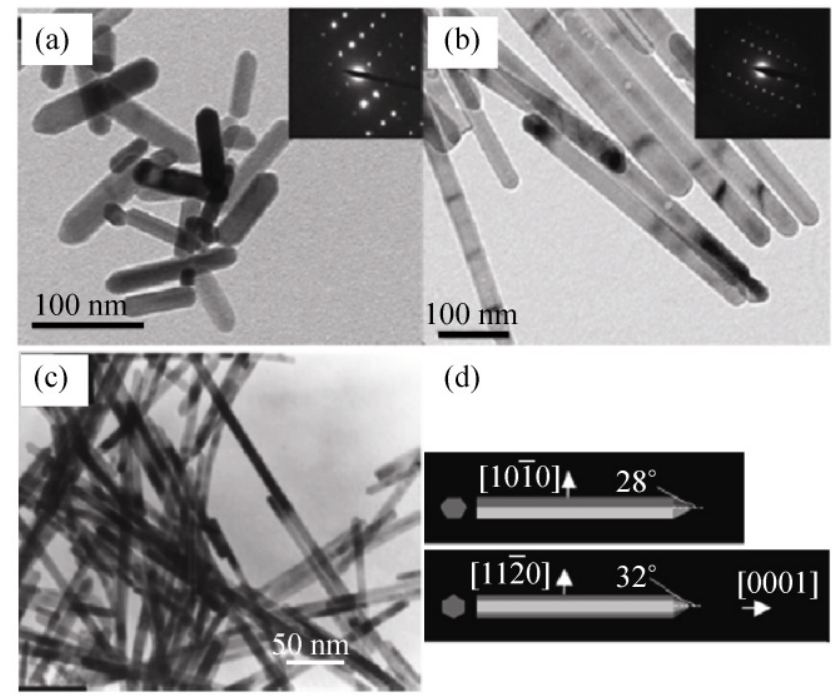

Figure 2 Transmission electron microscopy (TEM) images of $\mathrm{ZnO}$ nanowires synthesized in solvents having different polarities: (a) in methanol [66], (b) in ethanol [66], and (c) in 1-butanol [68] Even though the reaction temperature and the growth time are different, we can still see the effect of the solvent polarity on the nanowire aspect ratio. Insets in (a) and (b) are selected area electron diffraction patterns. (d) Schematic illustration of growing $+c$ ends of $\mathrm{ZnO}$ with two common interplanar angles [67]. Reproduced with permission

When the solvent contained nonpolar hexane, ultrathin $\mathrm{ZnO}$ nanowires of diameters of $2 \mathrm{~nm}$ could be synthesized from a simple acetate precursor, as shown in Fig. 3(a) [71]. These ultrathin nanowires also self-assembled into uniform stacks of nanowires aligned parallel to each other with respect to the long axis [71]. Near-UV absorption and photoluminescence measurements were able to determine that quantum confinement effects were present in these ultrathin nanowires, with an excitonic ground state of about $3.55 \mathrm{eV}$ [71]. The ultrathin nanowires were possibly grown by oriented coalescence of quantum dots, as shown in Fig. 3(b). Pacholski et al. suggested that oriented attachment of preformed quasi-spherical $\mathrm{ZnO}$ nanoparticles should be a major reaction path during the formation of single crystalline nanowires [72, 73]. The bottlenecks between the attached adjacent nanoparticles were later filled up and the nanowire surfaces were thus, smoothened by Ostwald ripening [72].

The alkaline solution could also be weak bases, such as $\mathrm{NH}_{3} \cdot \mathrm{H}_{2} \mathrm{O}$ and other amine compounds [74]. For examples, growth kinetics of $\mathrm{ZnO}$ nanowires in $\mathrm{NH}_{3} \cdot \mathrm{H}_{2} \mathrm{O}$ has been well studied in the Ref. [75]. Besides providing a basic environment, $\mathrm{NH}_{3} \cdot \mathrm{H}_{2} \mathrm{O}$ is also able to mediate heterogeneous nucleation of $\mathrm{ZnO}$ nanowires [75-78]. Experiments have shown that due to depletion of $\mathrm{Zn}^{2+}$ ions the growth of the $\mathrm{ZnO}$ nanowires normally slowed down with time and eventually arrived at growth-dissolution equilibrium for longer reaction times. This limitation can be overcome by adding additional $\mathrm{Zn}$ nitrate solution [79], or by replenishing the growth solution $[77,78,80]$. Under the mediation of $\mathrm{NH}_{3} \cdot \mathrm{H}_{2} \mathrm{O}$, however, $\mathrm{Zn}^{2+}$ could be stabilized through the reversible reaction shown in equation (8) below, thus, leading to a relatively low level of supersaturation being maintained in the solution. At the growth temperature (typically 70 $95^{\circ} \mathrm{C}$ ), this promoted only heterogeneous growth on the seeded substrate and suppressed the homogeneous nucleation in the bulk solution. That is also the reason that why after growth the bulk solution and reaction container usually remained clear without any precipitation. As the reaction proceeded, $\mathrm{Zn}^{2+}$ was gradually
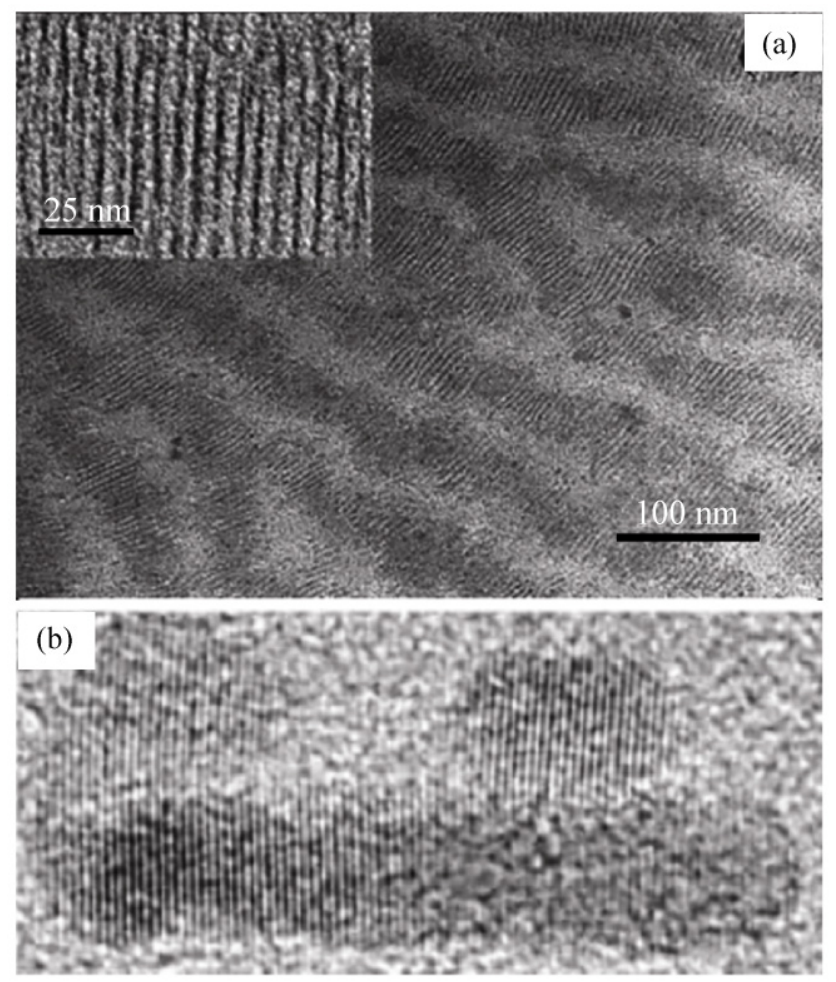

Figure 3 (a) TEM image of self-assembled $\mathrm{ZnO}$ nanowires with diameters of about $2 \mathrm{~nm}$ (inset: higher resolution image showing the oriented stacking; nanowires are dark contrast) [71]. (b) TEM image of the ultrathin nanowire formed by orientational aggregation of several quantum dots [72]. Reproduced with permission 
consumed and the zinc-ammonia complex gradually decomposed, thus, maintaining a stable level of $\mathrm{Zn}^{2+}$ in the solution. Therefore, all the reaction nutrient only contributed to the heterogeneous growth of $\mathrm{ZnO}$ nanowires on the seeded substrate, so the growth could last for a long time without replenishing the solution.

Equations (1) to (5) only describe a simplified version of the reaction processes. The actual scenario could be much more complicated than what has been discussed above. For example, oxygen molecules have not been considered at all, but in reality, the dissolved $\mathrm{O}_{2}$ concentration in the solution plays a significant role in the final crystal quality of the $\mathrm{ZnO}$ nanowires. There is experimental evidence showing that, if the growth solution was added with extra $\mathrm{H}_{2} \mathrm{O}_{2}$ that decomposed into $\mathrm{H}_{2} \mathrm{O}$ and $\mathrm{O}_{2}$, high quality $\mathrm{ZnO}$ nanowires with sharp top surfaces were grown [81]; if the solution was prepared with boiled de-ionized water to eliminate the dissolved $\mathrm{O}_{2}, \mathrm{ZnO}$ nanowires with very ragged surfaces were formed [82].

\subsection{Growth mediated by hexamethylenetetramine (HMTA) aqueous solution}

Probably the most commonly used chemical agents in the existing literature for the hydrothermal synthesis of $\mathrm{ZnO}$ nanowires are $\mathrm{Zn}\left(\mathrm{NO}_{3}\right)_{2}$ and HMTA $[83,84]$. In this case, $\mathrm{Zn}\left(\mathrm{NO}_{3}\right)_{2}$ provides $\mathrm{Zn}^{2+}$ ions required for building up $\mathrm{ZnO}$ nanowires. $\mathrm{H}_{2} \mathrm{O}$ molecules in the solution, unlike for the case of alkali-mediated growth, provide $\mathrm{O}^{2-}$ ions.

HMTA is a nonionic cyclic tertiary amine, as shown in Fig. 4. Even though the exact function of HMTA during the $\mathrm{ZnO}$ nanowire growth is still unclear, it has been suggested that it acts as a bidentate Lewis base that coordinates and bridges two $\mathrm{Zn}^{2+}$ ions [85]. So besides the inherent fast growth along direction of the polar surfaces of wurtzite $\mathrm{ZnO}$, attachment of HMTA to the nonpolar side facets also facilitates the

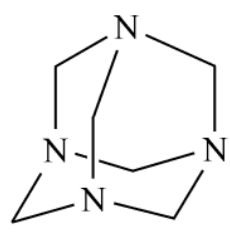

Figure 4 Molecular structure of HMTA anisotropic growth in the [0001] direction [86]. HMTA also acts as a weak base and $\mathrm{pH}$ buffer [49]. As shown in Fig. 4, HMTA is a rigid molecule, and it readily hydrolyzes in water and gradually produces $\mathrm{HCHO}$ and $\mathrm{NH}_{3}$, releasing the strain energy that is associated with its molecular structure, as shown in equations (6) and (7). This is critical in the synthesis process. If the HMTA simply hydrolyzed very quickly and produced a large amount of $\mathrm{OH}^{-}$in a short period of time, the $\mathrm{Zn}^{2+}$ ions in solution would precipitate out quickly owing to the high $\mathrm{pH}$ environment, and this eventually would result in fast consumption of the nutrient and prohibit the oriented growth of $\mathrm{ZnO}$ nanowires [87]. From reactions (8) and (9), $\mathrm{NH}_{3}$-the product of the decomposition of HMTA - plays two essential roles. First, it produces a basic environment that is necessary for the formation of $\mathrm{Zn}(\mathrm{OH})_{2}$. Second, it coordinates with $\mathrm{Zn}^{2+}$ and thus stabilizes the aqueous $\mathrm{Zn}^{2+} . \mathrm{Zn}(\mathrm{OH})_{2}$ dehydrates into $\mathrm{ZnO}$ when heated in an oven [84], in a microwave [88], under ultrasonication [89], or even under sunlight [90]. All five reactions (6) to (10) are actually in equilibrium and can be controlled by adjusting the reaction parameters, such as precursor concentration, growth temperature and growth time, pushing the reaction equilibrium forwards or backwards. In general, precursor concentration determines the nanowire density. Growth time and temperature control the $\mathrm{ZnO}$ nanowire morphology and aspect ratio $[50,91]$. As we can also see from equation (6), seven moles of reactants produce ten moles of products, so there is an increase in entropy during reaction, which means increasing the reaction temperature will push the equilibrium forwards. The rate of HMTA hydrolysis decreases with increasing $\mathrm{pH}$ and vice versa [49]. Note that the above five reactions proceed extremely slowly at room temperature. For example, when the precursor concentration is below $10 \mathrm{mmol} / \mathrm{L}$, the reaction solution remains transparent and clear for months at room temperature [82]. The reactions take place very fast if using microwaves as the heating source, and the average growth rate of the nanowires can be as high as $100 \mathrm{~nm} \cdot \mathrm{min}^{-1}$ [88].

$\mathrm{HMTA}+6 \mathrm{H}_{2} \mathrm{O} \longleftrightarrow 4 \mathrm{NH}_{3}+6 \mathrm{HCHO}$

$\mathrm{NH}_{3}+\mathrm{H}_{2} \mathrm{O} \longleftrightarrow \mathrm{NH}_{4}^{+}+\mathrm{OH}^{-}$ 


$$
\begin{aligned}
& \mathrm{Zn}^{2+}+4 \mathrm{NH}_{3} \longleftrightarrow\left[\mathrm{Z}\left(\mathrm{NH}_{3}\right)_{4}\right]^{2+} \\
& \mathrm{Zn}^{2+}+2 \mathrm{OH}^{-} \longleftrightarrow \mathrm{Zn}(\mathrm{OH})_{2} \\
& \mathrm{Zn}(\mathrm{OH})_{2} \longleftrightarrow \mathrm{ZnO}+\mathrm{H}_{2} \mathrm{O}
\end{aligned}
$$

Even though the counter-ions are not involved in the growth process according to these reaction equations, they have been shown to have a strong effect on the resulting morphology of $\mathrm{ZnO}$ nanowires [49]. Acetate, formate, and chloride mainly result in the formation of rods; nitrate and perchlorate mainly produce wires; and sulfate yields flat hexagonal platelets.

\subsection{Seeded growth on general substrates}

One main advantage of wet chemical methods is that, using $\mathrm{ZnO}$ seeds in the form of thin films or nanoparticles, $\mathrm{ZnO}$ nanowires can be grown on arbitrary substrates, such as Si wafers (flat [84], etched [82], and pillar array [92]), polydimethylsiloxane (PDMS) [93], thermoplastic polyurethanes (TPU) [94], paper [95], fibers [96, 97], and carbon fibers [98], as illustrated in Fig. 5. There has been a report of the dependence of nanowire growth rate on the $\mathrm{Si}$ substrate orientation, however [99]. The adhesion of the seed layer to the substrate is of critical importance, and can be improved by depositing an intermediate metal layer, such as $\mathrm{Cr}$ or Ti, on inorganic substrates [100], and by introducing an interfacial bonding layer, such as tetraethoxysilane molecules, on a polymer substrate [96]. Through the use of seeds, wafer-scale synthesis can be readily achieved $[88,93]$.

The seed thin film can be coated on the substrate prior to wet chemical growth [83, 84]. The seed layer can be prepared in a number of ways. Sputtering of bulk materials and spin coating of colloidal quantum dots are the two most commonly used methods [100102]. During the growth, $\mathrm{ZnO}$ nanowires preferentially nucleate from the cup tip near the grain boundaries between two adjacent grains in the $\mathrm{ZnO}$ seed film [103]. The width of the as-grown nanowires is usually less than $100 \mathrm{~nm}$, which is largely dictated by the grain size of the polycrystalline seeds. The length of the nanowires can be more than $10 \mu \mathrm{m}$, so the aspect ratio can be over 100 [104]. The $\mathrm{ZnO}$ seed layer has a random in-plane alignment, but generally has the $c$ axis per-

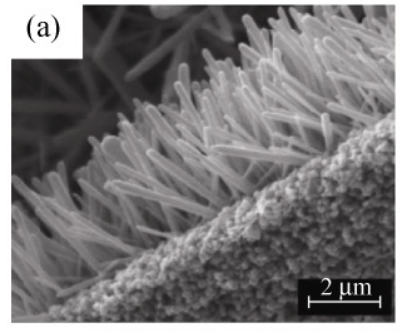

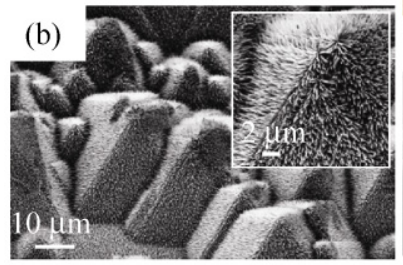

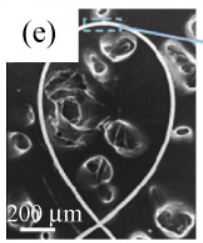

(h)

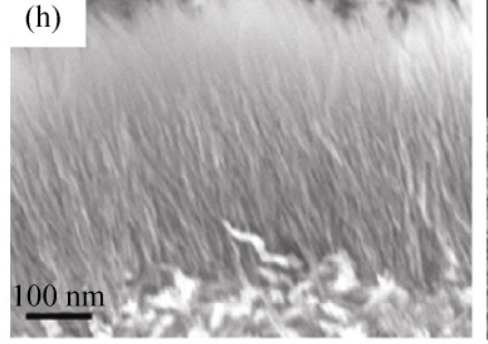

(f)
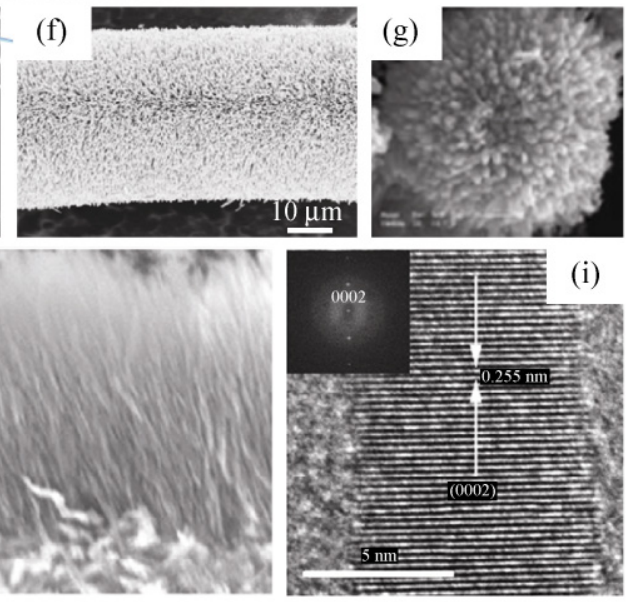

Figure 5 Scanning electron microscope (SEM) images of $\mathrm{ZnO}$ nanowire arrays grown on a $\mathrm{ZnO}$ seeded (a) flat rigid substrate [84], and (b) etched Si wafer (inset is an enlarged view) [82]. (c) Photograph of a four-inch flexible TPU substrate [94], and (d) SEM image of $\mathrm{ZnO}$ nanowire arrays with a uniform length on the TPU substrate [94]. (e) SEM image of a looped Kevlar fiber with $\mathrm{ZnO}$ nanowire arrays grown on top, showing the flexibility and strong binding of the nanowires [96], and (f) an enlarged local part of (e), showing a uniform distribution at the bending area [96]. (g) SEM image of $\mathrm{ZnO}$ nanowire arrays grown on a polystyrene sphere [107]. (h) Cross-section SEM image of ultrathin $\mathrm{ZnO}$ nanofibers grown on a $\mathrm{Zn}$ metal substrate [108]. (i) Highresolution transmission electron microscopy (HRTEM) image of a single $\mathrm{ZnO}$ nanofiber. Inset is the corresponding fast Fourier transform pattern [108]. Reproduced with permission

pendicular to the substrate [64], even though there have been occasions when there was non-perfect $c$ orientation [105]. The vertical alignment of the nanowire arrays is usually poor due to the polycrystalline nature of the seed $[83,84]$. Green et al. demonstrated that $\mathrm{ZnO}$ nanocrystal seeds prepared by thermal 
decomposition of a zinc acetate precursor could give vertically well-aligned $\mathrm{ZnO}$ nanowire arrays [106], and the degree of alignment depended strongly on the ambient humidity level during the seeding step [89].

$\mathrm{Zn}$ metal can also be the seed, because it is easily oxidized to $\mathrm{ZnO}$ in air and solution [77]. Fang et al. demonstrated an approach to synthesize dense arrays of ultrathin $\mathrm{ZnO}$ nanofibers using a $\mathrm{Zn}$ metal substrate in an ammonia/alcohol/water mixed solution [108], as shown in Fig. 5. As mentioned above, $\mathrm{ZnO}$ can grow in the absence of $\mathrm{H}_{2} \mathrm{O}$ using an alkaline medium. Studies by Kar et al. have shown that, in the presence of $\mathrm{NaOH}$ using ethanol as the sole solvent, different kinds of morphologies of $\mathrm{ZnO}$ could be synthesized on $\mathrm{Zn}$ foil, such as nanosheets, nanonails, and wellaligned nanorods [109]. In particular, the degree of alignment of the nanorods improved with the use of $\mathrm{NaOH}$ [109].

There is a competition between homogeneous nucleation and heterogeneous nucleation in solution, and heterogeneous nucleation generally has a lower activation energy barrier than homogeneous nucleation. Also, the interfacial energy between crystals and substrates is usually lower than that between crystals and solution [30]. Hence, heterogeneous growth on a seeded substrate occurs at lower levels of supersaturation than nucleation and growth in homogeneous solution $[49,76,83,110,111]$. In other words, growth on existing seeds is more favorable than nucleation in homogeneous solution for the reason that the existing seeds bypassed the nucleation step. Therefore, there will be growth of $\mathrm{ZnO}$ nanowires wherever there are $\mathrm{ZnO}$ seeds, and as a result the density of nanowires is typically quite high [84, 94-96]. Efforts have been made to control the density of the seeded $\mathrm{ZnO}$ nanowire arrays for applications such as field emission [112, 113], and direct current nanogenerators [100, 114].

In simple terms, controlling the seed layer thickness can control the nanowire density. The thickness of the seed layer could be small enough that the seeds no longer form a continuous thin film, but form separated islands. Liu et al. found that, when the seed layer thickness was changed from $1.5 \mathrm{~nm}$ to $3.5 \mathrm{~nm}$ by sputtering, the density of the $\mathrm{ZnO}$ arrays changed from $6.8 \times 10^{4}$ to $2.6 \times 10^{10}$ nanowires $/ \mathrm{cm}^{2}$ [112]. When the seed layer thickness was beyond this range, the nanowire density was less sensitive. If the seed layer was too thin, due to the high surface area and thus the high chemical potential of the polycrystalline seeds, dissolution exceeded deposition in the initial growth stage, and therefore no $\mathrm{ZnO}$ nanowires could be formed. If the thickness was larger than a certain value, e.g., $3.5 \mathrm{~nm}$, only the outermost layer of the seed played a role. If the seed layer was prepared by spin coating of colloidal dots, controlling the spin speed enabled control of the density of the colloidal dots on the substrate. By tuning the spin speed from 4000 to $8000 \mathrm{r} / \mathrm{min}$, the dot density changed from $(1.8 \pm 0.03) \times$ $10^{3}$ to $(1.8 \pm 0.03) \times 10^{2} \mathrm{dots} / \mu \mathrm{m}^{2}$, and consequently the $\mathrm{ZnO}$ nanowire array density varied from $(5.6 \pm 0.01) \times$ $10^{2}$ to $(1.2 \pm 0.01) \times 10^{2}$ nanowires $/ \mu \mathrm{m}^{2}$ [101]. Besides controlling the seed density, diffusion obstacle layers have also been applied to the $\mathrm{ZnO}$ seed layer in order to control the nanowire density [113]. For example, a thin blocking polymer film was established on top of the seed layer. In this way, the probability and rate of precursor molecules migrating from solution to the seed layer was adjusted. Therefore, the probability and density of the nucleation and eventual nanowire growth were effectively controlled.

\subsection{Electrodeposition}

Electrochemical deposition is a very powerful technique for achieving uniform and large area synthesis of $\mathrm{ZnO}$ nanostructures [115], because it exerts a strong external driving force to make the reactions take place, even if they are non-spontaneous. In this case, growth of $\mathrm{ZnO}$ nanostructures can occur on a general substrate, flat or curved [116], without any seeds, as long as the substrate is conductive. Also, under such an external electric field, better nanowire alignment and stronger adhesion to the substrate have been observed [117]. Generally speaking, $\mathrm{ZnO}$ nanowire growth was observed at only the cathode of a d.c. power source [117], and at both electrodes for an a.c. power source. Most importantly, electrodeposition has been shown to be an effective way of doping $\mathrm{ZnO}$ nanowires by adding different ingredients into the reaction solution [118-120].

For electrodeposition, a standard three-electrode setup is typically used, with a saturated $\mathrm{Ag} / \mathrm{AgCl}$ electrode as the reference electrode and $\mathrm{Pt}$ as the 
counter-electrode. The anode, where growth usually takes place, is placed parallel to the cathode in the deposition solution. The electrical bias throughout the reaction system is controlled by a constant voltage source to maintain a constant driving force to the reaction, or by a constant current source to keep a constant reaction rate. Konenkamp et al. used a $\mathrm{ZnCl}_{2}$ and $\mathrm{KCl}$ mixed solution electrolyte to grow vertically aligned $\mathrm{ZnO}$ nanowire arrays on a $\mathrm{SnO}_{2}$ glass substrate, as shown in Fig. 6 [118]. During the growth, $\mathrm{O}_{2}$ was continuously bubbled through the solution in order to keep a relatively high level of $\mathrm{O}_{2}$ dissolved in the solution, which was necessary for the growth of high quality $\mathrm{ZnO}$ nanowires as discussed above.

From equation (11), reduction of $\mathrm{O}_{2}$ at the cathode provides a source of $\mathrm{OH}^{-}$[121], which is required to coordinate with $\mathrm{Zn}^{2+}$ and then undergo dehydration to form $\mathrm{ZnO}$, as illustrated by equations (9) and (10). It has also been suggested that when using $\mathrm{Zn}\left(\mathrm{NO}_{3}\right)_{2}$ as the precursor, reduction of $\mathrm{NO}_{3}{ }^{-}$at the cathode could also provide a possible source of $\mathrm{OH}^{-}$[122], as indicated by equation (12). In any case, the ratio between the $\mathrm{OH}^{-}$generation rate at the cathode and the $\mathrm{Zn}^{2+}$ diffusion rate to the cathode was proposed to be the major parameter in the electrodeposition of $\mathrm{ZnO}$ nanowires [123]. Other than being produced in situ, $\mathrm{OH}^{-}$could also be added to the solution beforehand in the form of alkali precursors [122].

$$
\begin{aligned}
& \mathrm{O}_{2}+2 \mathrm{H}_{2} \mathrm{O}+4 \mathrm{e}^{-} \longleftrightarrow 4 \mathrm{OH}^{-} \\
& \mathrm{NO}_{3}^{-}+\mathrm{H}_{2} \mathrm{O}+2 \mathrm{e}^{-} \longleftrightarrow \mathrm{NO}_{2}^{-}+2 \mathrm{OH}^{-}
\end{aligned}
$$

$\mathrm{ZnCl}_{2}$ has commonly been used as the zinc source. It was found that the dimensions of $\mathrm{ZnO}$ nanowires could be controlled from 25 to $80 \mathrm{~nm}$ by the varying the $\mathrm{ZnCl}_{2}$ concentration [121]. Notably, $\mathrm{Cl}^{-}$ions became adsorbed preferentially on the Zn-terminated (0001) planes of $\mathrm{ZnO}$, which eventually hindered the growth along the polar axis, giving rise to platelet-like crystals [124], even though the anions are not considered as reactants according to equations (11) and (12). Even when other zinc salts rather than $\mathrm{ZnCl}_{2}$ were used as precursors, the $\mathrm{Cl}^{-}$could also come from the supporting electrolyte $\mathrm{KCl}$ [125]. Interestingly, although the electrolyte $\mathrm{KCl}$ was apparently not involved in the reaction, its concentration considerably affected the

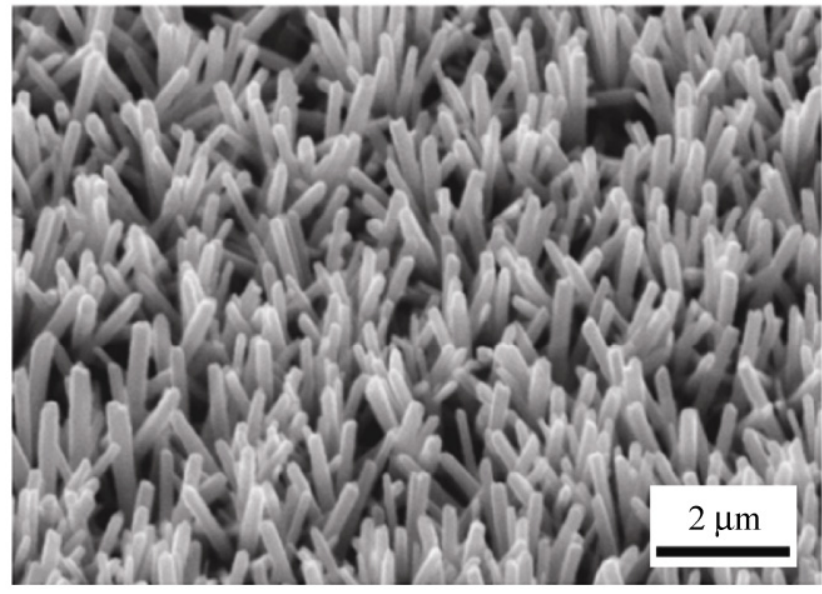

Figure 6 SEM image of free-standing $\mathrm{ZnO}$ nanowires formed on a $\mathrm{SnO}_{2}$ substrate by electrodeposition [118]. Reproduced with permission

reaction process. An increase in $\mathrm{KCl}$ concentration led to a decrease in the $\mathrm{O}_{2}$ reduction rate, and thus led to an augmentation of the growth efficiency of $\mathrm{ZnO}$ nanowires, which meant an enhancement of the axial growth rate relative to the radial growth rate. It has also been pointed out, however, that high $\mathrm{KCl}$ concentrations ( $>1 \mathrm{~mol} / \mathrm{L}$ ) also favored the radial growth of $\mathrm{ZnO}$ nanowires [125]. This effect was attributed to $\mathrm{Cl}^{-}$ion adsorption on the cathode surface, with a preferential adsorption of the $\mathrm{Cl}^{-}$on the (0001) ZnO surface [125]. In addition, the $\mathrm{KCl}$ concentration could also affect the lattice parameters of the as-synthesized $\mathrm{ZnO}$ nanostructures, especially for $\mathrm{KCl}$ concentrations $>1 \mathrm{~mol} / \mathrm{L}$ [126], and it was proposed that this was due to the inclusion of zinc interstitials in the lattice.

The effect of counter anions $\left(\mathrm{Cl}^{-}, \mathrm{SO}_{4}{ }^{2-}\right.$, and $\mathrm{CH}_{3} \mathrm{COO}^{-}$) on the reduction of dissolved $\mathrm{O}_{2}$ in the solution has been systematically investigated [123]. Different counter anions have considerably different coordination capabilities with the different crystal planes of $\mathrm{ZnO}$ nanowires. Therefore, the different adsorption behaviors of the anions can result in different morphologies and growth rates of the nanowires. It was also found that varying the counter anions could greatly tune the diameter $(65-110 \mathrm{~nm})$ and length $(1.0-3.4 \mu \mathrm{m})$ of the nanowires. In particular, the presence of $\mathrm{Cl}^{-}$and $\mathrm{CH}_{3} \mathrm{COO}^{-}$could produce $\mathrm{ZnO}$ nanowires with the lowest and highest aspect ratios, respectively [123].

The $\mathrm{ZnO}$ nanowire arrays showed high transmittance 
in the visible range due to the large electronic band gap. Interestingly, the band gap of the $\mathrm{ZnO}$ nanowire arrays could be tuned by simply changing the zinc precursor concentration during the electrodeposition [127].

\subsection{Templated growth}

$\mathrm{ZnO}$ nanowires can be grown by electrodeposition methods in combination with templates, such as anodic aluminum oxide (AAO), polycarbonate membranes, nano-channel glass, and porous films self-organized from diblock copolymers. In the literature, the most widely used template is probably AAO due to its simplicity and capability of large area fabrication [128]. After nanowire growth, the template can be chemically dissolved and leaving behind the free standing nanowires.

A typical fabrication process is as follows. The template is attached to the surface of a substrate, which can be flat or curved, flexible or rigid. Then the substrate together with the template is set to be the cathode of a d.c. power source. Under the electric field $\mathrm{Zn}^{2+}$ ions or intermediate $\mathrm{Zn}$ coordination species diffuse towards the cathode and into the pores of the template. $\mathrm{OH}^{-}$ions are simultaneously produced at the cathode according to equations (11) and (12). These two ions react and result in the growth of nanowires inside the pores of the template. After the pore is filled, nanowire arrays can be obtained by dissolution of the template membrane. This technique is not limited to $\mathrm{ZnO}$ nanowires and applies to the electrodeposition of general semiconductive oxide nanostructures. However, because both $\mathrm{ZnO}$ and $\mathrm{Al}_{2} \mathrm{O}_{3}$ are amphoteric oxides, it is technically difficult to selectively remove the $\mathrm{Al}_{2} \mathrm{O}_{3}$ membrane in the presence of $\mathrm{ZnO}$ nanowires. As an alternative, polycarbonate templates have been shown to be able to produce free standing $\mathrm{ZnO}$ nanowire arrays. As shown in Fig. 7, Zhou et al. demonstrated a simple polycarbonate template method to synthesize 1D oxide nanostructures [129], among which, the diameters of the $\mathrm{ZnO}$ nanowires could be tuned from 60 to $260 \mathrm{~nm}$, with lengths in the $\sim \mu \mathrm{m}$ range, by reliably and reproducibly controlling the template pore channel dimensions [129].

However, the key issue for semiconductor nanowires fabricated by this technique is the crystalline quality, which in most cases is not perfect. The resulting
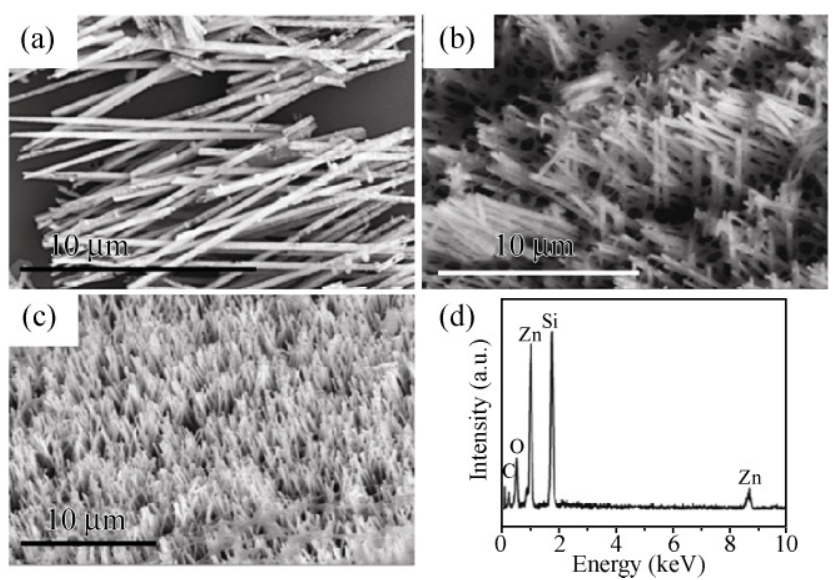

Figure 7 SEM images of (a) isolated $\mathrm{ZnO}$ nanowires, (b) $\mathrm{ZnO}$ nanowires embedded in a polycarbonate template, (c) free standing $\mathrm{ZnO}$ nanowire arrays after removal of the template, and (d) representative energy-dispersive X-ray spectroscopy (EDS) plot of the as prepared $\mathrm{ZnO}$ nanowire arrays [129]. Reproduced with permission

materials are either amorphous or polycrystalline consisting of small crystals with an abundance of defects, which might greatly limit their technical applications, particularly in optoelectronic devices. It is to be anticipated these shortcomings could be overcome by further optimizing the growth conditions.

Besides porous membranes, the templates could also be formed in situ inside the reaction system. Liu et al. showed that metallic $\mathrm{Zn}$ particles with their surface oxide coating could be a template for $\mathrm{ZnO}$ nanowire growth [130]. The reaction involves a so-called modified Kirkendall process in solution, where the preformed oxide layer serves as a shell template for the initial nucleation and growth [130]. Furthermore, selfassembled ionic polymers can also act a soft template for the growth of $\mathrm{ZnO}$ nanowires. Utilizing an Evans blue (EB) dye and a cetyltrimethylammonium bromide (CTAB) system, Cong et al. demonstrated a facile onestep process for the synthesis of a new kind of hybrid $\mathrm{ZnO}-$ dye hollow sphere made of aligned $\mathrm{ZnO}$ nanowires and dye molecules [131]. During the growth process, CTAB-EB micelles formed by an ionic selfassembly process served as a soft template for the deposition of ZnO [131-134]. In addition, Atanasova et al. demonstrated $\lambda$-DNA templated growth of $\mathrm{ZnO}$ nanowires, and the electrical resistance of the as-grown nanowires was found to be on the order of $\Omega$ [135].

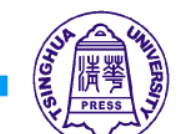

黑 Springer 


\subsection{Epitaxial growth}

Just as for seeded growth, epitaxial growth is also considered to involve a heterogeneous nucleation and growth process. Because of the small interfacial lattice mismatch, dangling bonds can be mostly satisfied and are less critical than for general interfaces. The energy benefits from satisfying the interfacial dangling bonds provide the driving force for the epitaxial growth. Different substrates have different isoelectric pointsthe $\mathrm{pH}$ where most sites on the substrate are neutral and the numbers of negative and positive sites are equivalent. So for an epitaxial substrate, positive or negative charge polarities should be considered as appropriate at different reaction $\mathrm{pH}$ values [136].

\subsubsection{Au coated general substrates}

While the formation of well-aligned $\mathrm{ZnO}$ nanowires on a pristine $\mathrm{Si}$ substrate is difficult because of a large mismatch ( $40 \%$ ) between $\mathrm{ZnO}$ and $\mathrm{Si}$, it is appealing to take advantage of the relatively small lattice mismatch between $\mathrm{ZnO}$ and other materials, such as $\mathrm{Au}$ [10, 137, 138], Pd [139], and Cu [140]. Figure 8(a) shows a crystal geometry diagram illustrating the epitaxial relationship of $\mathrm{ZnO}(0001)[11 \overline{2} 0] / / \mathrm{Au}(111)[\overline{1} 10]$, which have a lattice mismatch of $12.7 \%$ [141].

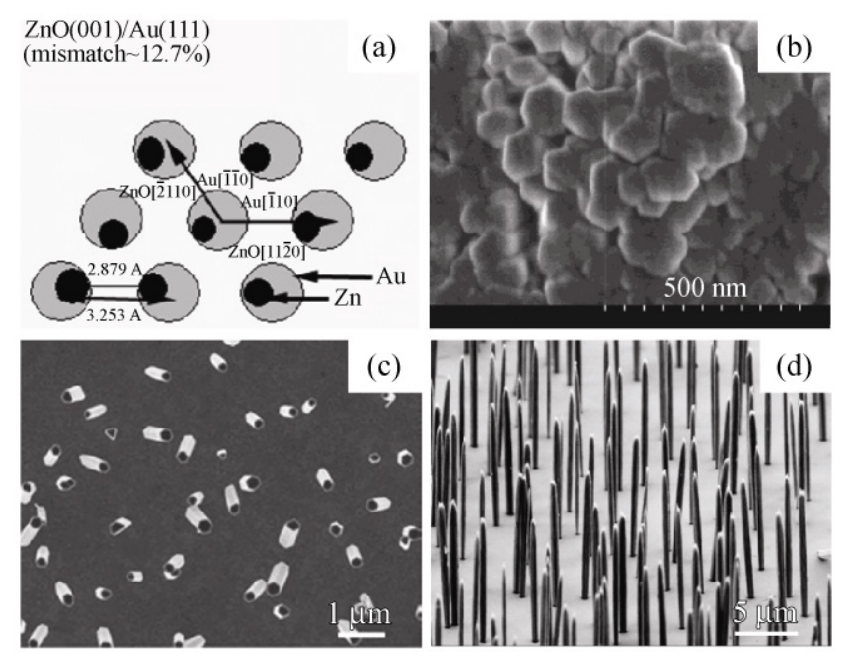

Figure 8 (a) Schematic illustration of the epitaxial relationship between $\mathrm{ZnO}(0001)$ and $\mathrm{Au}(111)$ [141]. SEM images of (b) 500-nmthick $\mathrm{ZnO}$ on single crystal $\mathrm{Au}(111)$ substrate [142], (c) density controlled $\mathrm{ZnO}$ nanowire arrays on polycrystalline $\mathrm{Au}(111)$ substrate [91], and (d) aspect ratio enhanced $\mathrm{ZnO}$ nanowire arrays guided by a statistical design of experiments [50]. Reproduced with permission
In the physical vapor deposition, Au was utilized as a catalyst in a vapor-liquid-solid process [32]. In wet chemical methods, Au is believed to be a mere epitaxial substrate [91]. $\mathrm{ZnO}$ nanowires have been electrodeposited epitaxially onto $\mathrm{Au}(111), \mathrm{Au}(110)$, and $\mathrm{Au}(100)$ single crystal substrates as shown in Fig. 8(b) [142]. The $\mathrm{ZnO}$ nanowire arrays were $c$-axis oriented, and had in-plane alignment, which was probed by X-ray pole analysis [142]. As can be seen from Fig. 8(b), the nanowires were very dense, almost continuous as a thin film. To replace the expensive single crystalline $\mathrm{Au}$, polycrystalline $\mathrm{Au}$ thin films coated on substrates such as $\mathrm{Si}$ wafers and flexible polymers were employed. As long as the substrate surface is locally flat to promote the vertical alignment of the $\mathrm{ZnO}$ nanowires [91], as shown in Fig. 8(c) [91, 143]. X-ray diffraction studies showed the as-deposited polycrystalline $\mathrm{Au}$ thin films were $<111>$ oriented normal to the substrate, even though they had random in-plane orientations [82]. The $<111>$ oriented Au film resulted in the growth of [0001] oriented $\mathrm{ZnO}$ nuclei due to the small lattice mismatch between them [141]. The density of the $\mathrm{ZnO}$ nanowires could be readily tuned and was found to be controlled by the concentration of the reactants, such as HMTA and $\mathrm{Zn}\left(\mathrm{NO}_{3}\right)_{2}$ [91]. The nanowire density increased with $\left[\mathrm{Zn}^{2+}\right]$ at low concentrations and decreased with $\left[\mathrm{Zn}^{2+}\right]$ at high concentration levels. The nanowire morphology was very sensitive to the growth temperature. When the temperature was increased from $70{ }^{\circ} \mathrm{C}$ to $95^{\circ} \mathrm{C}$, the nanowires transformed to nanopyramids, exposing the higher energy $\{01 \overline{1} 1\}$ surfaces [91]. This was probably due to the electrostatic interaction between the ions in the solution and the polar surfaces, and as a result higher Miller index surfaces became preferred [144]. One thing worth noting is that, by virtue of the surface tension, the substrate was put face-down floating on the nutrient solution [91], as shown in Fig. 9, to keep any precipitates from falling from the bulk solution onto the substrate, which would otherwise inhibit the growth of the desired nanostructures and possibly initiate secondary growth [91]. When the substrate was floating, it was suggested that the nuclei of $\mathrm{ZnO}$ were actually formed at the air-solution-substrate threephase boundaries and then migrated and settled down on the substrate [82]. 


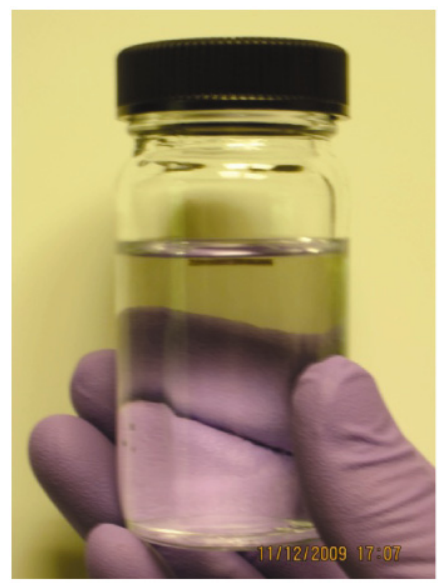

Figure 9 A digital photograph of the reaction container showing the substrate floating on the solution surface by surface tension

The aspect ratio is typically around 10 for $\mathrm{ZnO}$ nanowires grown on a polycrystalline $\mathrm{Au}$ thin film. A novel way of optimizing the nanowire aspect ratio by utilizing the statistical pick-the-winner rule and one-pair-at-a-time main effect analysis to sequentially design the experiments and identify optimal reaction settings has been demonstrated [50]. By controlling the hydrothermal reaction parameters, such as reaction temperature, time, precursor concentration, and possibly capping agent, the aspect ratio of $\mathrm{ZnO}$ nanowires was increased from around 10 to nearly 23, as shown in Fig. 8(d). These statistical design and analysis methods were very effective in reducing the number of experiments needed to be performed to identify the optimal experimental settings [50].

\subsection{2n-GaN/p-GaN}

Due to a small lattice mismatch, almost perfectly vertically aligned $\mathrm{ZnO}$ nanowire arrays can be grown on GaN (n-type [48] and p-type [145-149]), AlN, SiC, $\mathrm{Al}_{2} \mathrm{O}_{3}$, and $\mathrm{MgAl}_{2} \mathrm{O}_{4}$ substrates [150], either by hydrothermal decomposition [151] or electrodeposition. In particular, $\mathrm{ZnO}$ and $\mathrm{GaN}$ have the same wurtzite-type structure with a low lattice mismatch of $1.8 \%$ [152], which is much smaller than that $(12.7 \%)$ with $\mathrm{Au}(111)$. This is reflected by the much better vertical alignment of the $\mathrm{ZnO}$ nanowire arrays grown on $n$-type $\mathrm{GaN}(0001)$ (Figs. 10(a) and 10(b)) than on $\mathrm{Au}(111)$ (Figs. 8(c) and 8(d)). Nevertheless, the nanowires grown on both substrates have uniform length and width, and are well distributed on the substrates with one nanowire growing on one spot.

The epitaxial relationship between the as-grown $\mathrm{ZnO}$ nanowires and the $\mathrm{GaN}$ substrate is evidenced by X-ray diffraction (XRD) [136, 153]. Figure 10(c) shows a six-fold rotational symmetry in the azimuthal scan. The results clearly showed that the epitaxial nanowires had uniform vertical as well as in-plane alignment. If some of the nanowires have a different in-plane orientation, then the $\phi$-scan will be characterized by more than six peaks. The $\phi$-scan of the $\mathrm{ZnO}$ nanowires could be superimposed on the $\phi$-scan for the GaN substrate, which indicated a good epitaxial relationship between the as-grown $\mathrm{ZnO}$ nanowires and the $\mathrm{GaN}$ substrate. Furthermore, the small full width at half maximum of the diffraction peaks also showed a good crystalline quality [153].

\subsection{Capping agent-assisted growth}

Capping agents can be included in the solution to modify the growth habits of the $\mathrm{ZnO}$ nanostructures [154]. Commonly used capping agents for hydrothermal growth of $\mathrm{ZnO}$ nanostructures may be placed in two categories: those that adsorb onto the
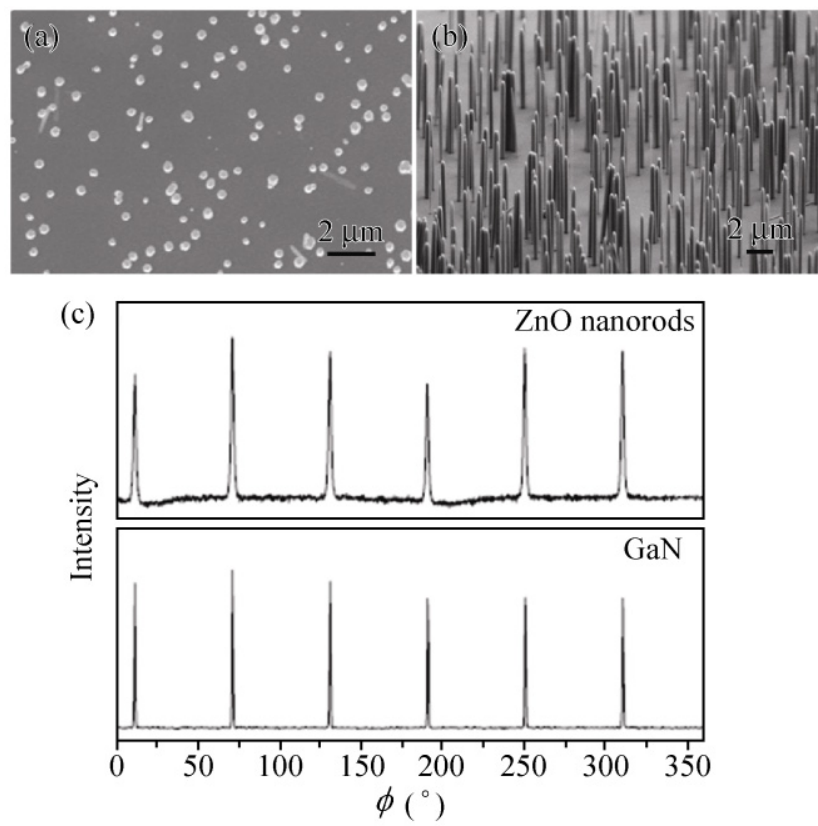

Figure 10 SEM images of $\mathrm{ZnO}$ nanowires on a $n$-type GaN wafer: (a) top view and (b) oblique view [82]. (c) $\phi$-scan profiles of the $\mathrm{ZnO}$ nanowires/GaN/c-sapphire structure with the family of planes of $\mathrm{ZnO}$ nanowires on the top, and the GaN film at the bottom [153]. Reproduced with permission 
side surfaces and enhance the vertical growth, such as amines like polyethylenimine (PEI) $[18,155,156]$ and ethylenediamine $[67,137]$; those that cap onto the basal plane of the $\mathrm{ZnO}$ nanostructures and promote lateral growth, such as $\mathrm{Cl}^{-}[124]$ and $\mathrm{C}_{3} \mathrm{H}_{5} \mathrm{O}(\mathrm{COO})_{3}{ }^{3-}$ (citrate ions) [150, 157, 158].

The isoelectric point of $\mathrm{ZnO}$ powder is at around $\mathrm{pH}=9.5$ [54]. The sign of the $\mathrm{ZnO}$ surface sites is predominately positive or negative for $\mathrm{pH}$ values below or above the isoelectric point, respectively [136]. PEI is a nonpolar polymer with a large amount of amino side-groups $\left(-\mathrm{NH}_{2}\right)$, which can be protonated over a wide range of $\mathrm{pH}$ values (3-11) and therefore become positively charged. The $\mathrm{pH}$ value of the growth solution could be adjusted to fall in the range that leads to the protonation of PEI, and therefore the linear PEI with its high positive charge density adsorbs strongly on the negatively charged surfaces due to electrostatic attraction [155], as shown in Fig. 11(a). Thus, the lateral growth of the nanowires will be largely hindered [156].

Other than their capping behavior, PEI additives also help to grow longer nanowires by extending the growth time [156], as shown in Fig. 11(b). This is similar to the effect of adding $\mathrm{NH}_{3} \cdot \mathrm{H}_{2} \mathrm{O}$ to increase the solubility of the nutrient precursor, and can be attributed to the decrease of the free $\left[\mathrm{Zn}^{2+}\right]$ that usually combines with $\mathrm{OH}^{-}$and precipitates in the form of $\mathrm{Zn}(\mathrm{OH})_{2}$, due to the coordination of PEI to $\mathrm{Zn}^{2+}$. Also after the growth, less precipitate was expected to form in the bulk solution in the presence of PEI coordination than without PEI. Thus, longer nanowire arrays could be produced through prolonging the growth time without refreshing the growth solution, because the $\mathrm{Zn}^{2+}$ depleted during the growth would be replenished through the decomposition of PEI- $\mathrm{Zn}^{2+}$ complexes [156].

Citrate ions are characterized by three negative charges under the normal growth environment. Experimental results in the literature as well as theoretical calculations suggest that citrate ions strongly and specifically adsorb to the $\mathrm{Zn}^{2+}$ ions on the (0001) surface, and thus inhibit the growth along [0001] and forced to grow along the $\langle 01 \overline{1} 0\rangle$ or $\langle 2 \overline{1} \overline{1} 0\rangle$ directions [150, 157, 160]. With citrate ions, rather than long hexagonal nanowires, flat hexagonal nanoplates were produced, as shown in Fig. 11(c) [150, 157]. Due to the high roughness factor and/or the large areas of
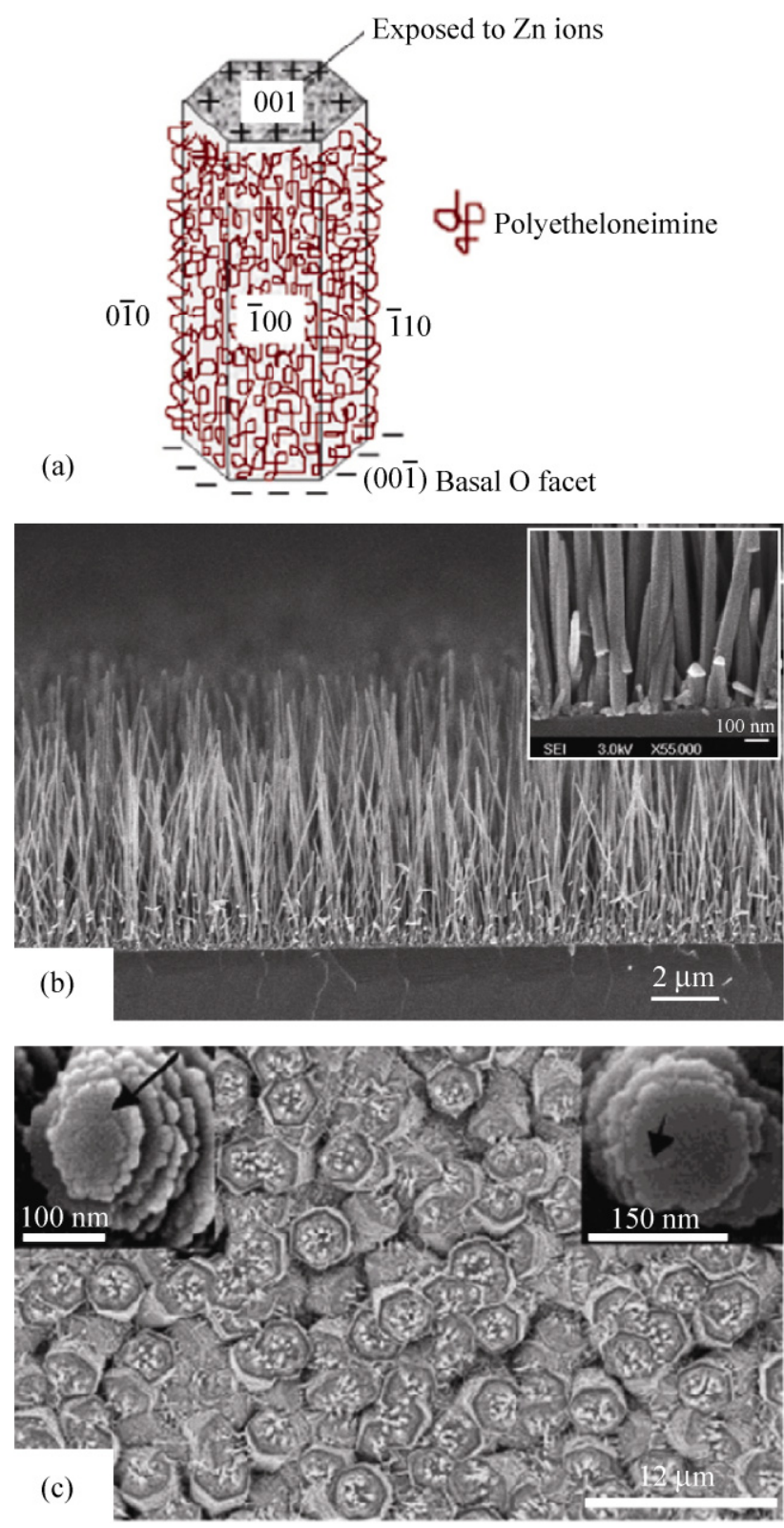

Figure 11 (a) Schematic illustration of the adsorption of PEI molecules on the $\mathrm{ZnO}$ nanowire side surfaces [159]. (b) SEM image of the $\mathrm{ZnO}$ nanowires formed with the addition of PEI [155]. (c) Large arrays of well-aligned helical $\mathrm{ZnO}$ whiskers on top of $\mathrm{ZnO}$ rod base [150, 157]. Reproduced with permission

exposed polar basal planes, the $\mathrm{ZnO}$ nanoplates showed enhanced photocatalytic properties for decomposition of volatile organic compounds in comparison with common 1D ZnO nanowire arrays [161]. Also, it was suggested that due to the presence of the citrate ions, the surface tension of the growth solution was reduced, which lowered the energy needed to form a new 
phase, and thus $\mathrm{ZnO}$ nanostructures could therefore nucleate at a lower supersaturation [158]. In addition to $\mathrm{ZnO}$, this synthetic approach can also be employed to modify and control the growth of other nanostructures such as conductive polymer nanowires [162] and $\mathrm{TiO}_{2}$ nanotubes [163].

\section{Different structures}

$\mathrm{ZnO}$ can be manipulated into a variety of forms and morphologies, including nanowires, nanobelts, tubes/rings, twinning structures, hierarchical structures, and heterostructures with other materials, showing the great versatility of wet chemical methods.

\subsection{Belts}

$\mathrm{ZnO}$ can grow along non-polar directions, such as $\langle 01 \overline{1} 0\rangle$ and $\langle 2 \overline{1} \overline{1} 0\rangle$, and form 1D nanobelts [31], which have high energy polar \pm (0001) side surfaces. This configuration has normally been observed using gas phase synthesis approaches [14, 31, 164-166], and is not favorable using aqueous approaches because-as discussed previously-wet chemical methods are usually considered to be under thermodynamic equilibrium, and the driving force is the minimization of the free energy of the entire reaction system [56]. However, there have been several reports of the synthesis of rectangular cross-sectional $\mathrm{ZnO}$ nanobelts by wet chemical methods, even though they were not necessarily growing along the non-polar directions. The nanobelts were free floating in the bulk solution [73] or standing on a substrate [167, 168].

Figure 12(a) shows a an SEM image of $\mathrm{ZnO}$ nanobelts synthesized in a microemulsion-mediated solution method [73]. The reverse micelles formed microreactors to confine the $\mathrm{ZnO}$ nanoparticles. Owing to the anisotropic growth property of wurtzite $\mathrm{ZnO}$, the nanoparticles undergo an oriented attachment process to lower the overall system energy by piling up and then fusing with the adjacent nanoplates. This eventually led to the formation of unique 1D rectangular cross-sectional $\mathrm{ZnO}$ nanobelts. This oriented attachmentbased growth mechanism dominated when the concentration of the precursors was high. The subsequent Ostwald ripening process smoothened out the resulting nanobelts when the precursor concentration was lowered [73]. Rectangular crosssection $\mathrm{ZnO}$ nanobelts, growing along the [0001] direction, have also been synthesized by a regular alkaline hydrothermal method with the help of ethylenediamine (Fig. 12(b)) [167]. Other than that, vertically aligned $\mathrm{ZnO}$ nanobelts on metallic $\mathrm{Zn}$ substrate have been fabrication by an in situ electrochemical method, as shown in Fig. 12(c). For the formation of $\mathrm{ZnO}$ nanowire or nanobelt arrays, it was suggested that the critical step is whether the nucleation is slow or fast, which leads to the formation of nanowires or nanobelts, respectively [168]. Porous $\mathrm{ZnO}$ nanobelts, shown in Fig. 12(d), were prepared from the thermal decomposition of synthetic bilayered basic zinc acetate nanobelts obtained by a simple synthetic route under mild conditions. During calcination in air, organic ligands and intercalated water molecules were removed from the synthetic bilayered basic zinc acetate nanobelts, leaving behind the inorganic porous $\mathrm{ZnO}$ nanobelts and nanoparticle chains. In essence, the synthetic bilayered basic zinc acetate nanobelts serve as templates [169].
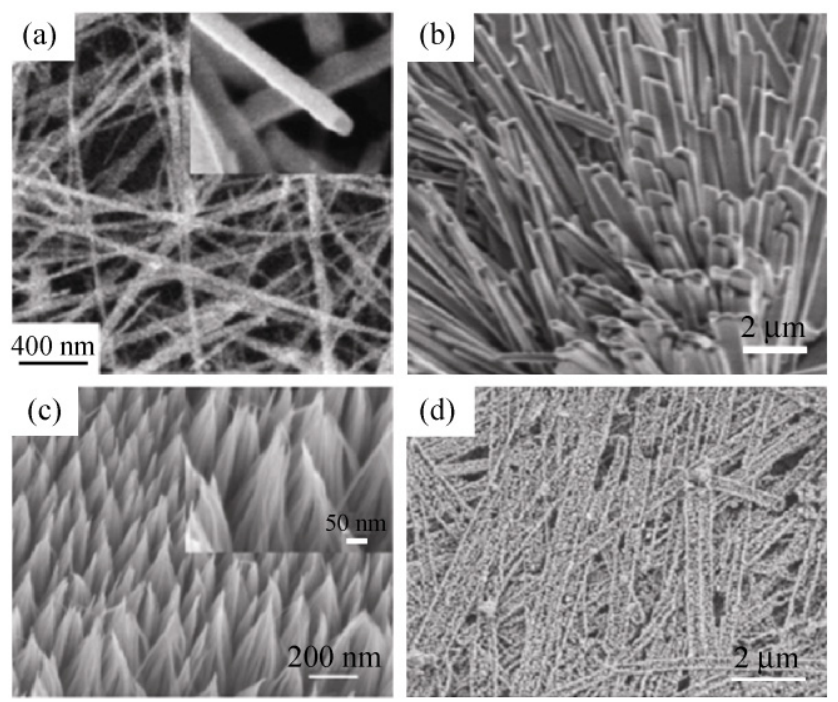

Figure 12 (a) SEM image of $\mathrm{ZnO}$ nanobelts synthesized by a microemulsion-mediated wet chemical method. Inset shows a typical rectangular cross-section feature of the nanobelts [73]. (b) SEM image of free standing nanobelt arrays [167]. (c) SEM image of $\mathrm{ZnO}$ nanobelt arrays grown on a metallic $\mathrm{Zn}$ substrate [168]. (d) SEM image of porous polycrystalline $\mathrm{ZnO}$ nanobelts and nanoparticle chains formed by the thermal decomposition of synthetic bilayered basic zinc acetate nanobelts [169]. Reproduced with permission 


\subsection{Tubes/rings}

Tubular structures are of particular interest for many potential applications, such as in high efficiency solar cells due to the high internal surface area relative to nanowires, and in novel bimolecular or gas sensors due to the well-defined adsorption microcavities [170]. $\mathrm{ZnO}$ nanotubes have been fabricated using a variety of approaches [170-172], such as optimizing the seed layer thickness [173], utilizing appropriate solvent composition [174], ultrasonic pretreatment of the reaction solution [175], and post $\mathrm{pH}$ adjustment [176]. The $\mathrm{ZnO}$ nanotubes can be made by one-step growth methods [117, 174], or two-step growth and etching processes [177, 178], as shown in Fig. 13. The nanotube wall thickness can be precisely controlled by controlling the electrodeposition time [179]. The nanotube could also be grown on large scale on seeded general substrates [180], with a yield approaching $100 \%$ [181].

Different formation mechanisms have been proposed in the literature. For example, She et al. proposed that the tubular morphology was formed via the defect selective etching of $\mathrm{ZnO}$ nanowires on the polar surface by protons generated from anodic water splitting [172]. The high energy (0001) basal plane of $\mathrm{ZnO}$ nanowire results in preferential etching in the (0001) direction [104]. The distribution of point defects on the basal plane of the $\mathrm{ZnO}$ nanowire was rather non-uniform with the center higher than the peripheral part. Therefore, the center part was etched away faster than the peripheral part, which led to tubular morphology. This hypothesis was indirectly supported by a control experiment in which annealed $\mathrm{ZnO}$ nanowires could not be etched to give nanotubes [172]. Also, it is suggested that different termination atomszinc or oxygen - on the (0001) basal plane play a decisive role in the formation of nanotubes or nanowires [182]. Yu et al. proposed that the formation of $\mathrm{ZnO}$ nanotubes arose from the low concentration of precursor molecules during electrodeposition [117]. The electric field around the edge was stronger than that right above the hexagonal basal plane, so the limited precursor molecules would preferentially drift to the edge where as a result growth was faster than at the center. However, $\mathrm{Li}$ et al. ascribed the formation (a)
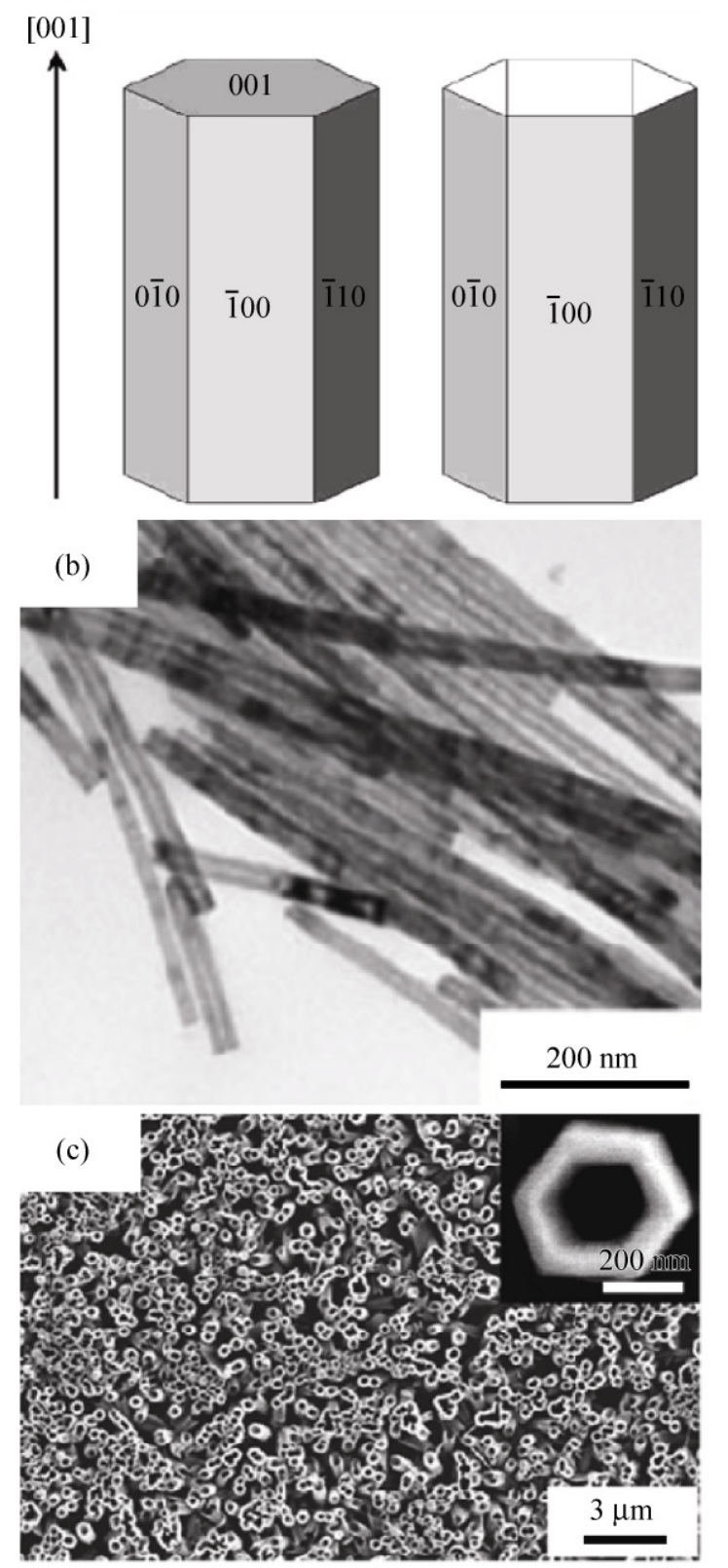

Figure 13 (a) Crystal growth habit of wurtzite $\mathrm{ZnO}$ hexagonal nanowires and nanotubes [170]. (b) TEM image of high aspect ratio ultrathin single crystalline $\mathrm{ZnO}$ nanotubes [173]. (c) SEM image of arrayed $\mathrm{ZnO}$ nanotubes [172]. Reproduced with permission

of nanotubes to the incorporation during the initial growth, and subsequent dissolution, of nitrogencontaining organic compounds at the core of the $\mathrm{ZnO}$ nanowires [183]. Yang et al. also suggested a scrolling of layer structures as the mechanism of formation of the nanotubes [178].

Nevertheless, it is accepted that the formation of 
$\mathrm{ZnO}$ nanotubes is a kinetically controlled process. The final morphology and dimension of the nanostructures are determined by a competition between adsorption and desorption of the precursor molecules, or in other words crystal growth and dissolution processes [56, 184]. At the initial stage, the growth rate is relatively high because of the high supersaturation degree of growth nutrient. With prolonged hydrothermal treatment, the reaction reaches a certain equilibrium, and the solution composition is no longer thermodynamically favorable for formation of $\mathrm{Zn}(\mathrm{OH})_{2}$ that can subsequently dehydrate into $\mathrm{ZnO}$ [87], and the rate of $\mathrm{ZnO}$ dissolution is faster than the rate of formation [174]. As discussed previously, the polar surfaces will be dissolved preferentially since this decreases the system energy during the subsequent aging process, and that gradually leads to the formation of $\mathrm{ZnO}$ nanotubes [183, 185], as illustrated in Fig. 14 [186].

In a further step, $\mathrm{ZnO}$ rings could also be synthesized by the growth of plates and a subsequent etching process [187]. Li et al. used sodium bis(2-ethylhexyl)sulfosuccinate (NaAOT) as surfactant/ template to fabricate $\mathrm{ZnO}$ rings and disks at low temperature on a large scale, as shown in Fig. 5(a) [187]. The anionic surfactant NaAOT can form micelles/microreactors with diverse shapes from spheres to rods, ellipsoids, and disks by adjusting the experimental parameters [188]. $\mathrm{ZnO}$ nanostructures can grow in the resulting microreactors. The self-
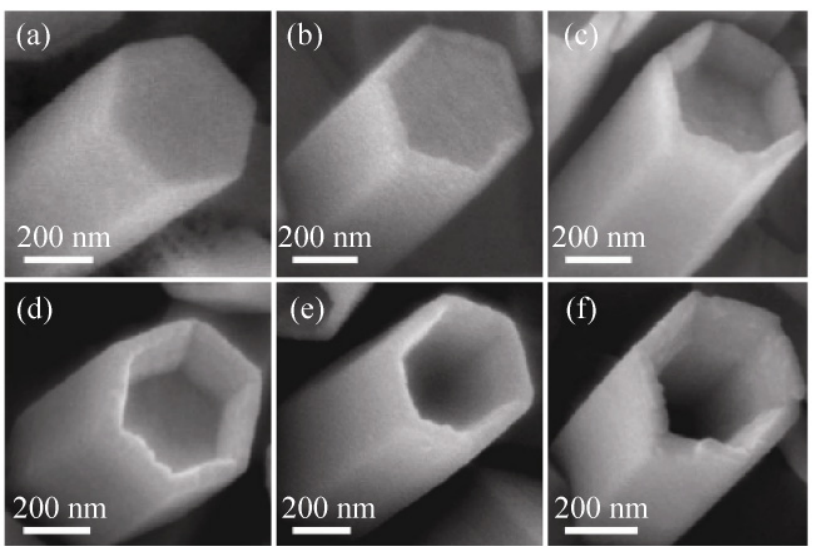

Figure 14 SEM images illustrating the formation of $\mathrm{ZnO}$ nanotubes at different etching stages: (a) $0 \mathrm{~min}$, (b) $5 \mathrm{~min}$, (c) $10 \mathrm{~min}$, (d) $15 \mathrm{~min}$, (e) $60 \mathrm{~min}$, and (f) $120 \mathrm{~min}$ [186]. Reproduced with permission assembled AOT ions at the water/oil interface can attract $\mathrm{Zn}^{2+}$ ions and thus direct the nucleation of the $\mathrm{ZnO}$. By controlling the growth parameters, such as the growth temperature and molar ratio of reactants, the nanodisks can be converted into rings due to the electrostatic interaction between the anionic AOT ions and the $\mathrm{Zn}^{2+}$ ions on the (0001) surface of $\mathrm{ZnO}$, which therefore inhibits the growth along the [0001] direction, and promotes growth along $\langle 2 \overline{1} \overline{1} 0\rangle$, forming hexagonal nanodisks enclosed by $\{10 \overline{1} 0\}$ side surfaces. A subsequent etching leads to the formation of hexagonal nanorings [187].

In addition to the growth and etching processes, a self-template directed growth process was shown to lead to fabrication of $\mathrm{ZnO}$ rings, as shown in Fig. 15(b) [189]. By a simple solvothermal method, the atypically shaped coordination polymer particles were formed by the cooperation of two different organic ligands, namely $N, N^{\prime}$-phenylenebis(salicylideneimine)dicarboxylic acid (A) and 1,4-benzenedicarboxylic acid (B). The growth mechanism is shown in Fig. 15(c) [190]. These two ligands formed coordination polymer disks, which later served as templates for the formation of the $\mathrm{Zn}^{2+}$ precursor shell, followed by dissolution of the coordination polymer disks. Calcination of the disks together with the $\mathrm{Zn}^{2+}$ precursor shell gave rise to $\mathrm{ZnO}$ rings, which was polycrystalline in nature [190].

\subsection{Twinning}

Twinned structures are very commonly formed by wet chemical methods [83, 111, 191-193]. Typical twinned $\mathrm{ZnO}$ structures [174] are shown in Fig. 16. Two joined hexagonal prisms connected by a common basal plane forming a twinning growth relationship. TEM studies showed that the $\mathrm{ZnO}$ is a single crystal with the oriented growth direction along the [0001] direction. A growth mechanism was proposed based on the linkage/incorporation of the growth units. Wang et al. suggested that the twinning relationship of the two branches should be altered in different growth solutions [194]. In pure water or weakly basic solutions, the twinned species are bipyramidal and take $(000 \overline{1})$ as the common connection plane. In contrast, when the growth solution contained $\mathrm{KBr}$ or $\mathrm{NaNO}_{2}$ as mineralizers, the twinning morphologies were dumbbell-like 

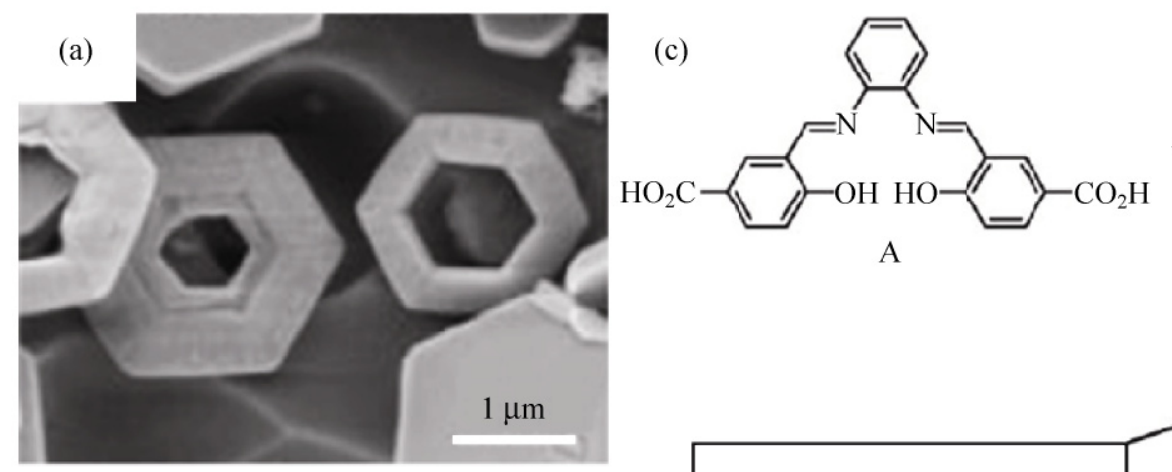

(b)

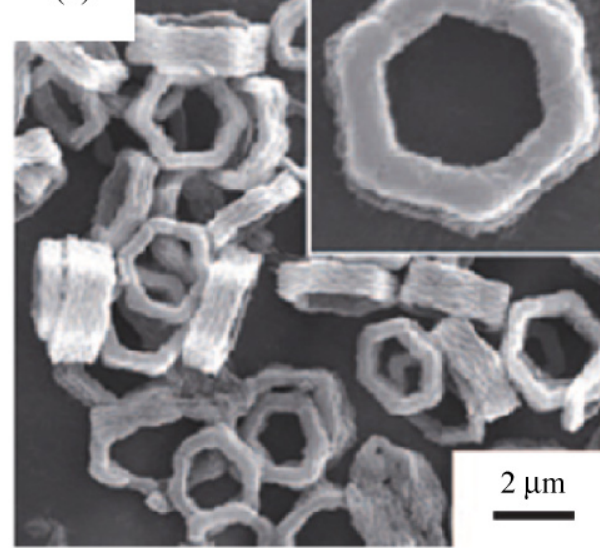

A

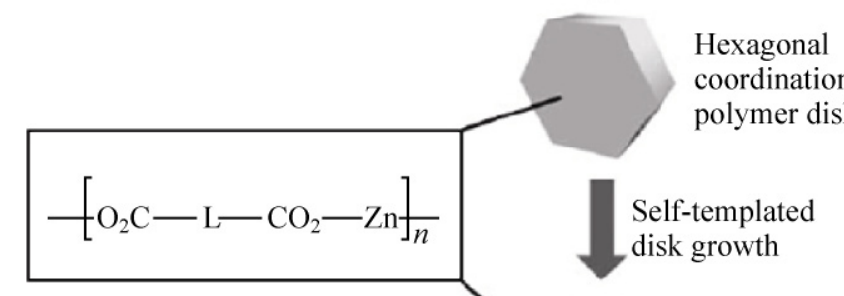<smiles>O=C(O)c1ccc(C(=O)O)cc1</smiles>

$\mathrm{Zn}(\mathrm{OAc})_{2}$ DMF, $120^{\circ} \mathrm{C}$ polymer disk

Hexagonal $\mathrm{ZnO}$ ring

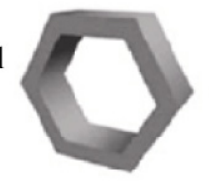

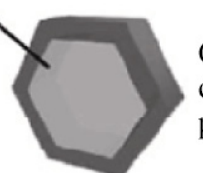

Coaxial coordination polymer disk

Template dissolution
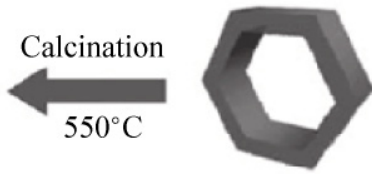

Hexagonal coordination polymer ring

Figure 15 (a) SEM image of the as synthesized $\mathrm{ZnO}$ rings prepared by the growth and etching process [187]. (b) SEM image of the templated growth and calcination of $\mathrm{ZnO}$ rings [189], and (c) the proposed growth mechanism, where ${ }^{-} \mathrm{O}_{2} \mathrm{C}-\mathrm{L}-\mathrm{CO}_{2}{ }^{-}$represents deprotonated acid A or B [190]. Reproduced with permission

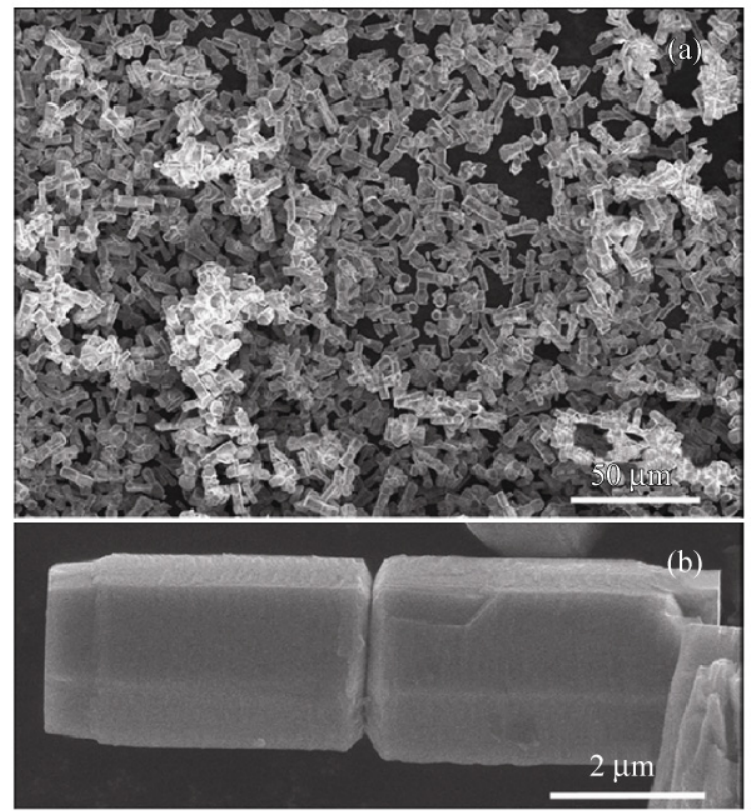

Figure 16 (a) A general view of the as-grown twinned $\mathrm{ZnO}$ structures, and (b) a magnified view of a symmetric twinned structure [174]. Reproduced with permission and took (0001) as the common connection plane. For the latter case, the $\mathrm{K}^{+}$or $\mathrm{Na}^{+}$ions might act as a bridge between the $\mathrm{ZnO}_{4}{ }^{{ }^{-}}$tetrahedral growth units, similar to the structure of mica [194]. In any case, the twinned structure is clearly favorable under specific reaction conditions [174].

\subsection{Hierarchical structures}

It is attractive to fabricate complex three-dimensional nanostructures with controlled morphology and orientation. Tian et al. demonstrated a strategic facial wet chemical method to synthesize well-controlled complex and oriented $\mathrm{ZnO}$ nanostructures [161]. Based on the conventional seeded growth of $\mathrm{ZnO}$ nanowires, they used citrate anion as capping agent, which has three negative charges and preferentially adsorbs onto the $\mathrm{ZnO}$ basal planes [160], which greatly inhibits the growth along the [0001] direction, resulting in the formation of thin nanoplatelets, as shown in Figs. 17(a) 
and 17(b). The as-grown $\mathrm{ZnO}$ columnar nanoplatelets were remarkably similar to the nacreous plate structures in red abalone fish shown in Figs. 17(c) and 17(d).

In conjunction with citrate anions, diamines-such as ethylene diamine, diaminobutane, and diaminopropane - have also been used to direct the growth, leading to initiation of secondary nucleation on the $\mathrm{ZnO}$ nanowire side surfaces [195-198]. The growth mechanism was proposed to involve their effect on the solution $\mathrm{pH}$ and coordination with $\mathrm{Zn}^{2+}$ [198]. Similar secondary nucleation on the $\mathrm{ZnO}$ nanowire side surfaces has also recently been demonstrated by coating with another round of seed nanoparticles [199]. Since the diaminopropane molecules combine with water molecules and release hydroxide ions, the preferred adsorption of the diaminopropane molecules on the nanowire side surfaces is expected to raise the local $\mathrm{pH}$ value, which assists the formation of the first a few layers of $\mathrm{ZnO}$ clusters near the side surfaces. These nucleated clusters perform as seeds to initiate the following vertical growth of the secondary branches on the side surfaces of the primary nanowires. When a small amount of diaminopropane was used, sparse and tapered $\mathrm{ZnO}$ nanotips grew randomly. As the amount of diaminopropane was increased, dense and more organized tapered $\mathrm{ZnO}$ nanotips resulted, almost covering the entire side surfaces [195].
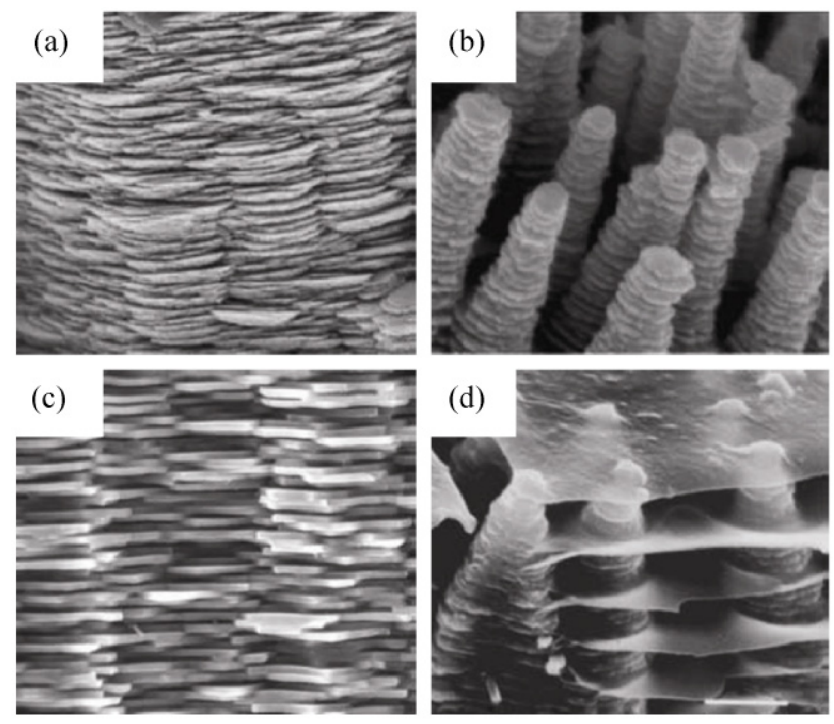

Figure 17 (a) Side view and (b) oblique view of oriented biomimetic $\mathrm{ZnO}$ columnar nanoplates, which resemble the (c) side view and (d) oblique view of the nacreous plate structures in red abalone fish [161]. Reproduced with permission
By rationally alternating the order of addition of the citrate and the diaminopropane, Zhang et al. have demonstrated a full capability to fabricate hierarchically oriented and ordered complex $\mathrm{ZnO}$ nanostructures step by step by wet chemical methods, as shown in Fig. 18 [195]. They conducted a series of systematic growth procedures to reveal the roles and the growth kinetics of these two organic structure-directing agents in the formation of secondary and tertiary $\mathrm{ZnO}$ nanoplates and nanobranches on selected facets of $\mathrm{ZnO}$ nanowires. As shown in Fig. 18(a), using the as-grown common vertical $\mathrm{ZnO}$ nanowires as the platform, when diaminopropane was added to initiate the secondary growth, side branches were nucleated all over the side surfaces of the hexagonal prism. Subsequently, using the as-grown secondary nanostructures, with citrate added to initiate tertiary growth, nanoplates formed on both the primary prism and the side branches. They also switched the sequence of addition of the two structure-directing agents, and the results were just as expected, as shown in Figs. 18(d)18(f) [195].

Other hierarchical structures, such as microspheres composed of radial $\mathrm{ZnO}$ nanowire arrays, have also been made by thermolysis of zinc and ethylenediamine complex precursors in the presence of poly(sodium 4-styrenesulfonate) (PSS), as shown in Fig. 19(a) [200]. It was suggested that the growth process starts with the PSS stabilized colloidal nanoclusters. The nanoclusters later aggregate into larger secondary spherical particles in order to minimize their surface energy. Then these secondary spherical particles further collide and merge with each other to form multimers (e.g., dimers, trimers, etc) by random Brownian motion. By Ostwald ripening, the hollow hemispheres made of $\mathrm{ZnO}$ nanowire arrays were formed by dissolving small multimers. Liu et al. reported a different approach towards hollow spherical $\mathrm{ZnO}$ aggregates, as shown in Figs. 19(b) and 19(c) [201]. The self-assembly of $\mathrm{ZnO}$ hollow spherical structures was made by the coordination of CTAB, $\mathrm{Zn}\left(\mathrm{NO}_{3}\right)_{2} \cdot 6 \mathrm{H}_{2} \mathrm{O}$, and ethylenediamine. The initial oriented attachment of the nanorods to the central stems [202] was followed by the formation of complex multi-pod units, and finally the construction of spheres from the multi-pod units. In other words, this hierarchical organization process 


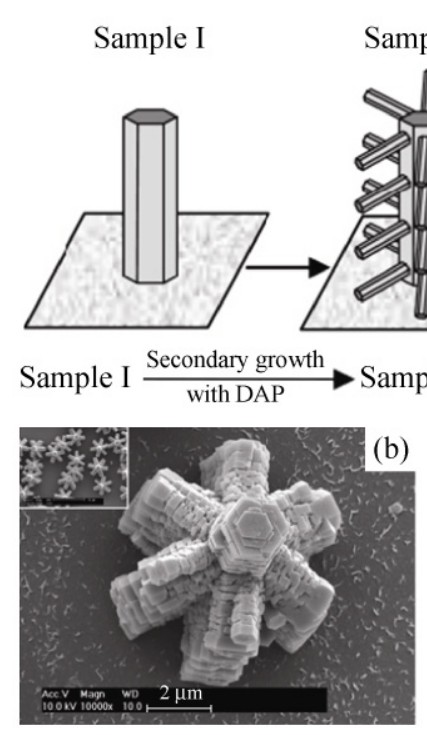

Sample II

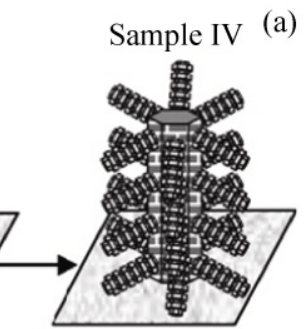

(a)
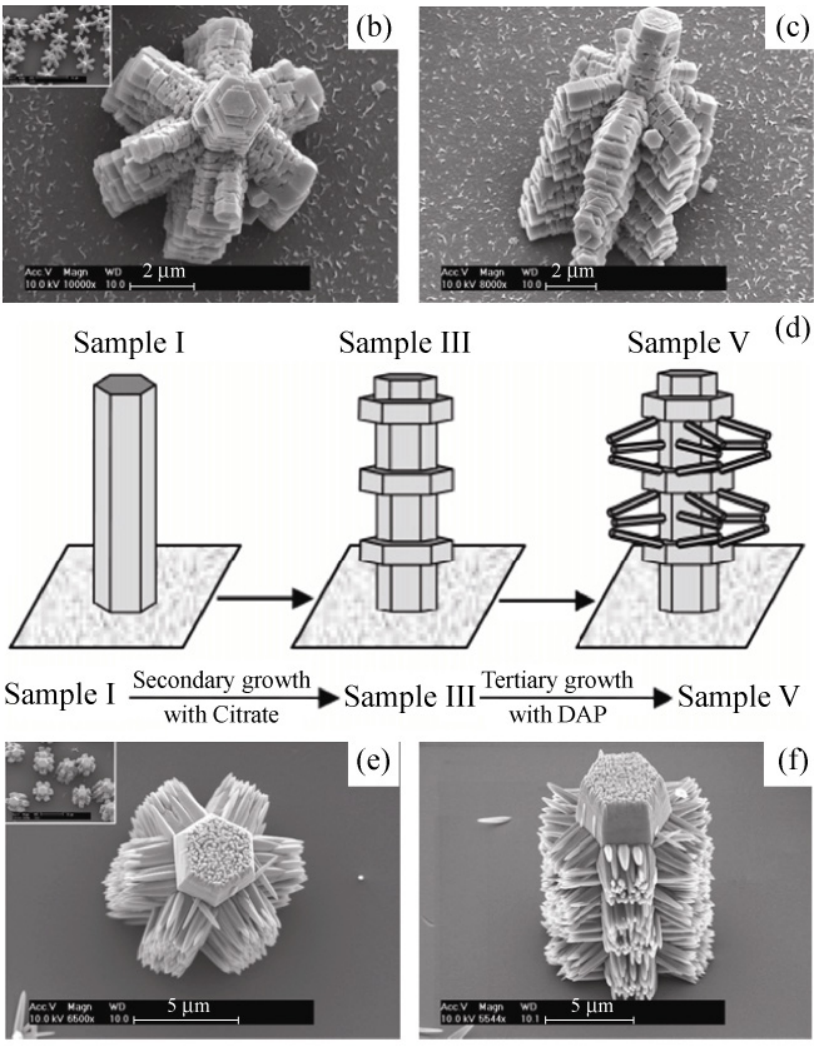

(d)
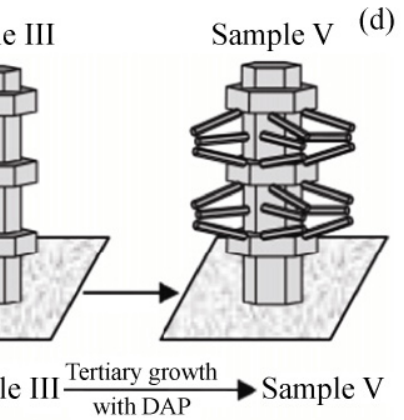

)

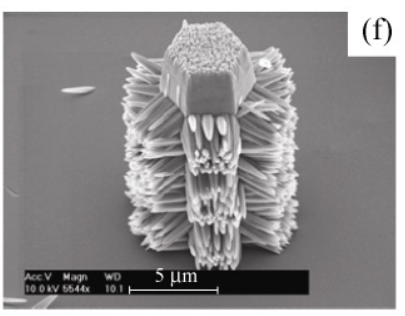

Figure 18 (a) Schematic illustration of the effect of consecutive addition of diaminopropane and citrate on the growth of hierarchical $\mathrm{ZnO}$ nanostructures, and (b) and (c) the corresponding SEM images of the as-grown nanostructures. (d) Schematic illustration of consecutive addition of citrate and diaminopropane on the growth of hierarchical $\mathrm{ZnO}$ nanostructures, and (e) and (f) the corresponding SEM images of the as-grown nanostructures [195]. Reproduced with permission

started from the generation of $\mathrm{ZnO}$ nanorods, then the nanorods became aggregated into multipod units, and finally the multipod units aggregated into the hollow microspheres [201].

Besides microspheres, layered $\mathrm{ZnO}$ nanowire arrays have been formed by wet chemical methods. Chow et al. and Koh et al. presented a template-free selfassembly of densely packed bilayered $\mathrm{ZnO}$ nanowire
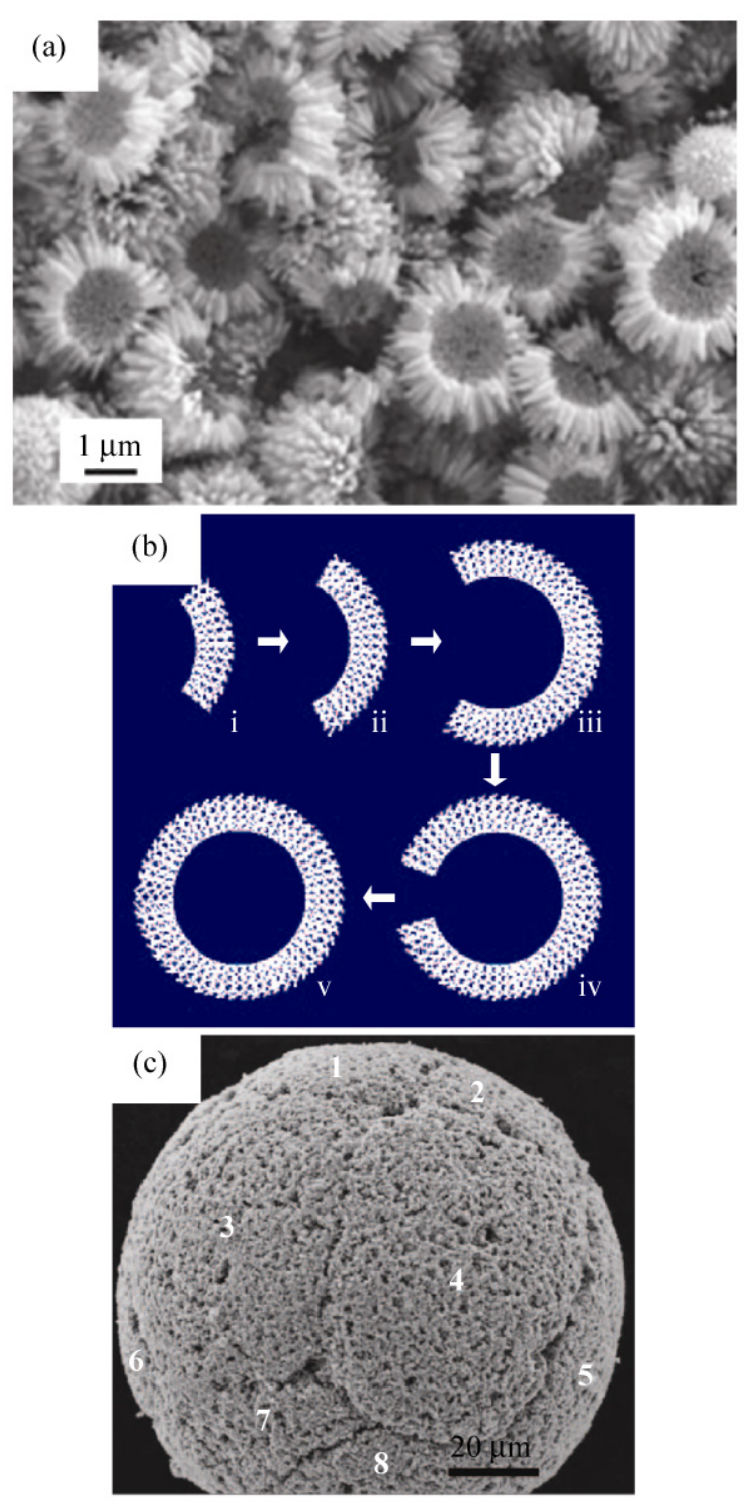

Figure 19 (a) SEM image of the hollow hemispheres selfassembled from $\mathrm{ZnO}$ nanowire arrays [200]. (b) Schematic illustration of the self-assembly process of the hollow microspheres, and (c) SEM image of a self-assembled hollow microsphere [201]. Reproduced with permission

arrays, as shown in Fig. 20(a) [203, 204]. In an alkaline environment, a thin layer of hydrotalcite-like zincowoodwardite plates was first formed as a result of the reaction between zinc and aluminum ions [203, 205, 206]. Zincowoodwardite belongs to a family of layered compounds with positively charged layers of $\mathrm{Zn}^{2+}$ and $\mathrm{Al}^{3+}$ with hydroxide anions, and interlayer charge-balancing anions $\mathrm{SO}_{4}{ }^{2-}$. It has a lattice constant of $3.076 \AA$ in the basal plane which has about $5 \%$ lattice mismatch with the basal plane of the wurtzite 
ZnO (3.249 A). Therefore the hydrotalcite-like plates can provide an epitaxial substrate for the oriented growth of well-aligned $\mathrm{ZnO}$ nanowire arrays on both the top and the bottom surfaces. The size of the nanowires could be controlled by changing the $\mathrm{pH}$ value of the solution [203]. Heterogeneous nucleation on the hydrotalcite-like plates requires a low interfacial energy, and is more favorable than the homogeneous nucleation of $\mathrm{ZnO}$ nanowires in the bulk solution $[203,204]$. Furthermore, delamination of the thin hydrotalcite-like plate at high temperatures produced free standing sheets of $\mathrm{ZnO}$ nanowire bundles [203]. Bilayer structured $\mathrm{ZnO}$ nanowire arrays were also formed by a $\mathrm{ZnO}$ thin film seeded growth method, as shown in Fig. 20(b) [207]. As the growth proceeded, growth nutrient was consumed, and the precursor concentration became dilute, which induced secondary growth and a decrease in the nanowire diameter and eventually the formation of bilayered structures [207].

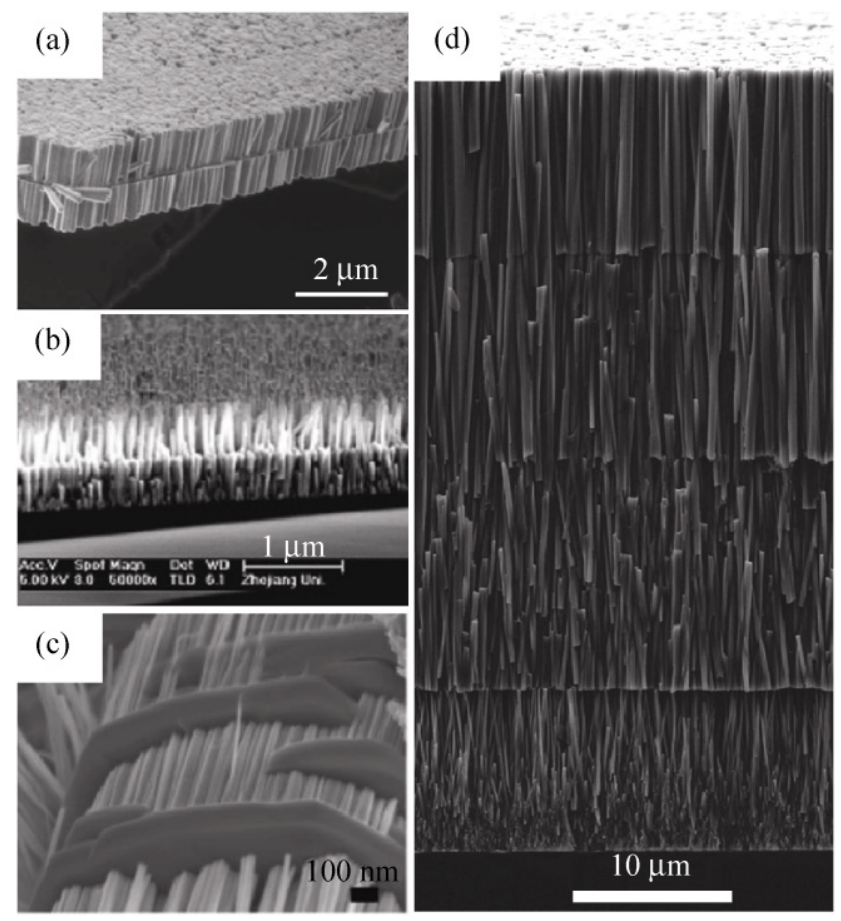

Figure 20 (a) SEM image of bilayered densely packed $\mathrm{ZnO}$ nanowire arrays on both sides of hydrotalcite-like zincowoodwardite plates [204]. (b) SEM image of bilayered $\mathrm{ZnO}$ nanowire arrays induced by secondary growth [207]. (c) SEM image of multilayered $\mathrm{ZnO}$ nanowire arrays sandwiched between parallel disks [208]. (d) SEM image of four-layered $\mathrm{ZnO}$ nanowire arrays [209]. Reproduced with permission
Li et al. demonstrated multilayered $\mathrm{ZnO}$ nanowire hierarchical structures, as shown in Fig. 20(c) [208]. The $\mathrm{ZnO}$ nanowire arrays were connected between parallel $\mathrm{ZnO}$ hexagonal disks. The reaction was conducted through a temperature-dependent multistep process. The precursor $\left[\mathrm{Zn}\left(\mathrm{NH}_{3}\right)_{4}\right]^{2+}$ underwent hydrolysis and dehydration to form nuclei for brucitetype polynuclear lamellar zinc hydroxide hexagonal disks, which later served as an intermediate substrate for the growth of $\mathrm{ZnO}$ nanowires. At the same time, as the temperature increased, the metastable zinc hydroxide disks dehydrated and transformed into $\mathrm{ZnO}$ disks. During dehydration, many regular nanosized $\mathrm{ZnO}$ islands were left on the disk surface, which seeded the secondary growth of $\mathrm{ZnO}$ nanowires, resulting in multicell sandwiches or nanowire-disk-nanowire-disk superlattices, as shown in Fig. 20(c) [208]. Most recently, $\mathrm{Xu}$ et al. demonstrated a novel technique for multilayer $\mathrm{ZnO}$ nanowire assemblies [209]. They coated a hydrophobic self-assembled monolayer (SAM) on the primary $\mathrm{ZnO}$ nanowires. Then by ultraviolet ozone treatment, the nanowire top surfaces were selectively exposed to refreshed solution resulting in additional growth, while the nanowire side surfaces were protected by the SAM from widening and fusing together. This process could be repeated multiple times, and a four-layer $\mathrm{ZnO}$ nanowire assembly was achieved as shown in Fig. 20(d). Finally, for device applications, all of the SAM coating could be removed by calcination.

Liu et al. demonstrated a templateless approach to grow hierarchical $\mathrm{ZnO}$ nanowire arrays with steplike heights on a common metallic zinc substrate [210] using equimolar $\mathrm{Zn}\left(\mathrm{ClO}_{4}\right)_{2}$ and $L$-cysteine solutions with a $\mathrm{pH}$ value of 10 . The metallic zinc substrate was vertically immersed into the solution without sealing. The reaction took place at room temperature over 3 days. The solution gradually evaporated, and therefore the vertical $\mathrm{Zn}$ substrate was gradually exposed to the air from positions I to $\mathrm{V}$, as illustrated in Fig. 21. The growth time of the $\mathrm{ZnO}$ nanowires in the solution increased from positions $\mathrm{I}$ to $\mathrm{V}$, and therefore the nanowires had different lengths. The length of the $\mathrm{ZnO}$ nanowires showed a nearly linear relationship with the position on the $\mathrm{Zn}$ substrate. However, the reason for stepwise growth rather than a gradual increase of the nanowire length is not clear. 

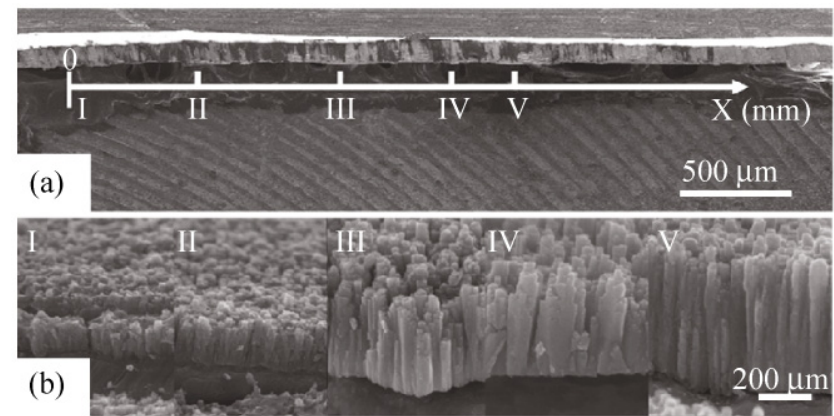

Figure 21 (a) Gradational growth of $1 \mathrm{D} \mathrm{ZnO}$ nanowire arrays on a common zinc substrate from left to right, and (b) crosssectional SEM images of the $1 \mathrm{D} \mathrm{ZnO}$ nanowire arrays at five different locations [210]. Reproduced with permission

\subsection{Heterostructures}

Each single component material has its own functions and also limitations. For future multifunctional nanosystems, it is necessary to integrate many different materials in a rational manner. Heterostructures of nanomaterials are a preliminary step towards this application.

\subsubsection{ZnO compound semiconductors}

It is possible to passivate the surface dangling bonds of $\mathrm{ZnO}$ nanowires by coating with other compound semiconductors, such as CdSe $[19,211,212]$ and CdTe [213] by electrodeposition, $\mathrm{CdS}$ [214], $\mathrm{SnO}_{2}$ [215], $\mathrm{MgO}$ [216, 217], and $\mathrm{ZrO}$ [217] by hydrothermal reaction, $\mathrm{Co}_{3} \mathrm{O}_{4}[218]$ by photochemical reaction, $\mathrm{ZnS}[219,220]$ by sulfidation, and $\mathrm{Al}_{2} \mathrm{O}_{3}$ [221] and $\mathrm{TiO}_{2}$ [222] by atomic layer deposition (ALD). In most cases, the asformed shell layers are polycrystalline or amorphous in nature.

CdSe was electrodeposited onto $\mathrm{ZnO}$ nanowires from an aqueous solution of $\mathrm{N}\left(\mathrm{CH}_{2} \mathrm{COOK}\right)_{3}, \mathrm{CdSO}_{4}$ and $\mathrm{Na}_{2} \mathrm{SeSO}_{3}$ at room temperature [211]. The $\mathrm{N}\left(\mathrm{CH}_{2} \mathrm{COOK}\right)_{3}$ acted as a complexing agent whilst $\mathrm{CdSO}_{4}$ and $\mathrm{Na}_{2} \mathrm{SeSO}_{3}$ were the sources of $\mathrm{Cd}$ and Se, respectively. During the electrodeposition, the $\mathrm{ZnO}$ nanowire sample was set to be the cathode, and $\mathrm{Pt}$ was the counter electrode. As shown in Fig. 22(a), the as-deposited CdSe layer uniformly covered the $\mathrm{ZnO}$ nanowires. The CdSe layer thickness could be increased by increasing the deposition current density and prolonging the deposition time [212]. X-ray diffraction studies showed the CdSe was polycrystalline in the cubic sphalerite phase [19]. Annealing should eliminate the organic components and also improve the shell crystallinity for further photovoltaic applications [19].

CdTe has a high optical absorption coefficient, a narrow band gap, and forms a typical type II band alignment with $\mathrm{ZnO}$, which renders it an excellent inorganic semiconductor sensitizer for photovoltaic
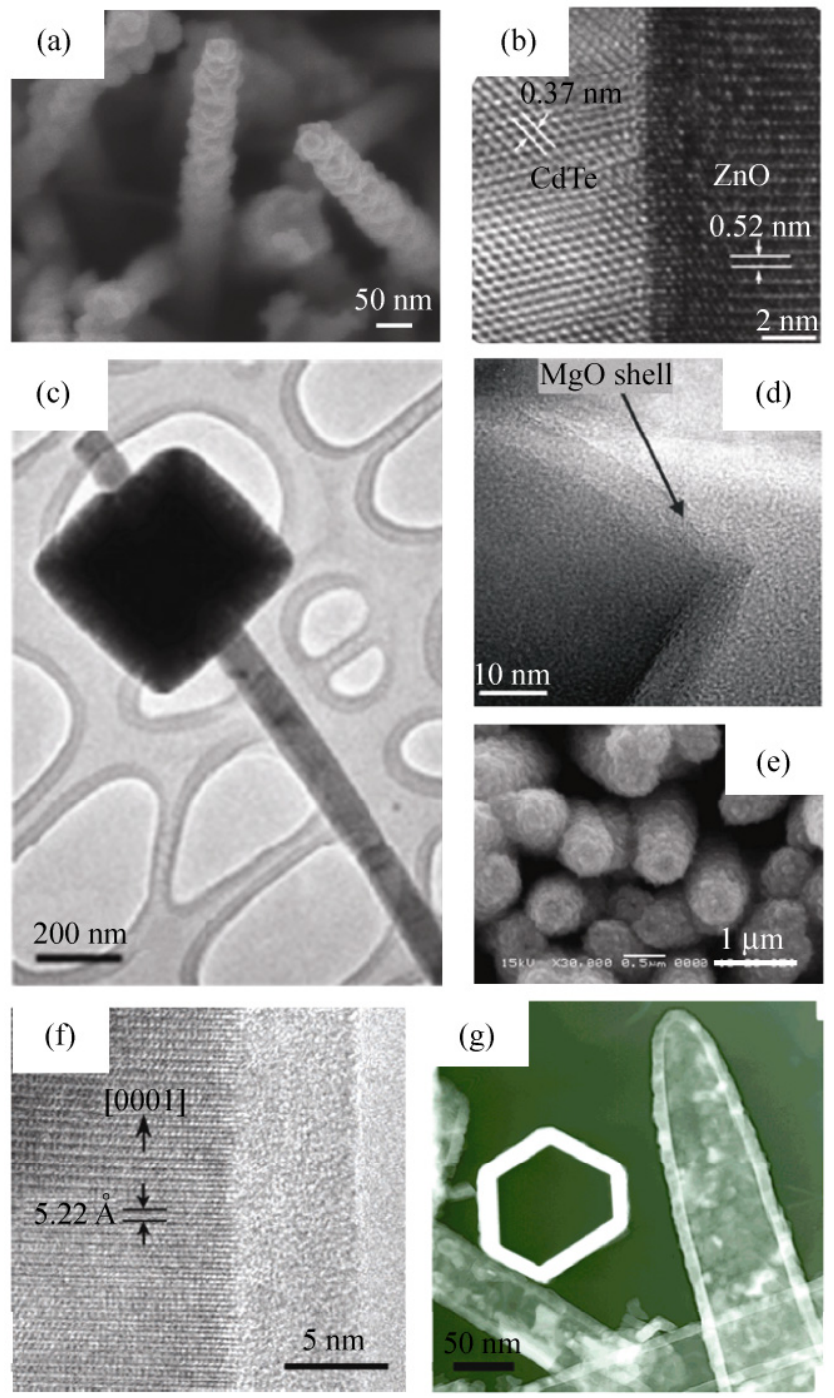

Figure 22 (a) Top view of $\mathrm{ZnO}$ nanowires covered with a CdSe thin film [211]. (b) HRTEM image taken from the $\mathrm{ZnO} / \mathrm{CdTe}$ interface region, showing the well-crystallized structure of the CdTe layer [213]. (c) Low magnification TEM image showing $\mathrm{SnO}_{2}$ capping a $\mathrm{ZnO}$ nanowire with the nanowire tip exposed [215]. (d) TEM image of a $\mathrm{ZnO}-\mathrm{MgO}$ core-shell nanowire [216]. (e) SEM image of cable-like $\mathrm{ZnS}-\mathrm{ZnO}$ nanowire structures [219]. (f) HRTEM image of the interface of a $\mathrm{ZnO} / \mathrm{Al}_{2} \mathrm{O}_{3}$ core-shell nanowire [221]. (g) Negative TEM image of an anatase $\mathrm{TiO}_{2}$ nanotube formed by etching away the $\mathrm{ZnO}$ nanowire core in $1 \mathrm{~mol} / \mathrm{L}$ aqueous $\mathrm{HCl}$ [222]. Reproduced with permission 
applications. The traditional reaction conditions for fabricating CdTe are too stringent to allow the presence of $\mathrm{ZnO}$ nanowires [223-226]. Wang et al. demonstrated the deposition of large-scale $\mathrm{ZnO}-\mathrm{CdTe}$ core-shell nanowire arrays on indium-tin-oxide (ITO) substrates through a benign electrodeposition method ( $\mathrm{pH} 8.3$ ), which was compatible with the $\mathrm{ZnO}$ nanowires [213]. The electrodeposition was carried out with a threeelectrode system, with the $\mathrm{ZnO}$ nanowire array as the working electrode, a standard calomel electrode (SCE) as the reference electrode, and a $\mathrm{Pt}$ foil as the counter electrode. The as-deposited CdTe shell was uniform in thickness, and could be tuned from several tens to hundreds of nanometers by changing the deposition time and the current density. As shown in Fig. 22(b), after deposition, an intact interface was formed between the single crystal $\mathrm{ZnO}$ nanowire core and the high crystallinity CdTe shell. XRD results showed that the CdTe shell had a zinc-blende structure, and the crystallinity could be further increased by annealing [213].

$\mathrm{SnO}_{2}$ has a wide band gap of $3.6 \mathrm{eV}$. Indium or fluorine doped $\mathrm{SnO}_{2}$ have been widely explored and used in industry as transparent electrodes. Shi et al. reported the capping of $\mathrm{ZnO}$ nanowires with doped $\mathrm{SnO}_{2}$, which could act as the top electrode of the $\mathrm{ZnO}$ nanowires [215]. The wet chemical growth of the $\mathrm{SnO}_{2}$ cap was conducted in a solution of $\mathrm{SnCl}_{4} \cdot 5 \mathrm{H}_{2} \mathrm{O}$, ethanol, and distilled water with a $\mathrm{pH}$ of 12 . Under such a high $\mathrm{pH}$ environment, the as-grown $\mathrm{ZnO}$ nanowires in the first step may have dissolved, and later reformed on the $\mathrm{Zn}$ substrate on which $\mathrm{SnO}_{2}$ caps were formed, as shown in Fig. 22(c). The similar photoluminescence spectra of the $\mathrm{ZnO}$ nanowire arrays before and after the $\mathrm{SnO}_{2}$ capping indicate that the electronic and optical qualities of the inner $\mathrm{ZnO}$ nanowire were not degraded after the secondary solution growth. From the cathode luminescence spectrum, the near-band-edge emission was enhanced and the defective deep level emission was suppressed after the capping. $\mathrm{SnO}_{2}$ has a larger band gap than $\mathrm{ZnO}$, which confines the electrons/holes in the $\mathrm{ZnO}$ nanowires more efficiently and thus leads to high internal quantum efficiency. In addition, the surface states of the $\mathrm{ZnO}$ nanowires (dangling bonds and/or surface defects) could be partially reduced via the capping surface passivation. The cap/nanowire configuration also allowed a direct measurement on the single nanowire junction $\left(\mathrm{Zn} / \mathrm{ZnO} / \mathrm{SnO}_{2}\right)$. The $I-V$ curve indicated there was a small barrier between the $\mathrm{Zn}$ substrate and the $\mathrm{ZnO}$ nanowire, and the $\mathrm{SnO}_{2} / \mathrm{ZnO}$ interface did not introduce any barrier [215]. In contrast, using $\mathrm{SnO}_{2}$ nanowires prepared by vapor phase deposition, Cheng et al. reported the seeded growth of $\mathrm{ZnO} / \mathrm{SnO}_{2}$ nanowires and, interestingly, random lasing behavior was observed from the heterostructures [227].

Plank et al. demonstrated a low temperature wet chemical method to coat a $\mathrm{MgO}$ shell layer onto $\mathrm{ZnO}$ nanowires that did not require a subsequent high temperature annealing [216]. In their process, the $\mathrm{ZnO}-\mathrm{MgO}$ core-shell nanowire structures were fabricated by submerging the $\mathrm{ZnO}$ nanowires in a mixed solution of $\mathrm{Mg}\left(\mathrm{NO}_{3}\right)_{2}$ and $\mathrm{NaOH}$. The thickness of the as-coated $\mathrm{MgO}$ layer could be controlled up to $8 \mathrm{~nm}$, as shown by the TEM image in Fig. 22(d) [216]. Electrons could efficiently tunnel through the "insulating" $\mathrm{MgO}$ shell. The $\mathrm{ZnO}-\mathrm{MgO}$ core-shell nanowires showed an enhanced efficiency in hybrid photovoltaic devices by enhancing the photoinduced charge generation [217] and the photocurrent and open circuit voltage [216]. Similarly, a $\mathrm{ZrO}_{2}$ shell could also be deposited on the $\mathrm{ZnO}$ nanowires by replacing the magnesium nitrate with zirconium acetate as precursor [217].

The synthesis of sulfide compounds is usually challenging, and a strongly reducing environment is required, because sulfides are easily oxidized into oxides. Wang et al. demonstrated a wet chemical approach to fabricate $\mathrm{ZnO}-\mathrm{ZnS}$ core-shell nanowire arrays by secondary sulfidation of $\mathrm{ZnO}$ nanowires [219]. In their method, they simply immersed the as-grown $\mathrm{ZnO}$ nanowire arrays in a Teflon autoclave containing an aqueous solution of thioacetamide. By controlling the reaction time, the product could be controlled to be pure $\mathrm{ZnS}$ nanotube arrays or $\mathrm{ZnO}$ $\mathrm{ZnS}$ nanocables with various $\mathrm{ZnO}$-to-ZnS ratios. The growth mechanism was suggested to be non-epitaxial, and involve ion exchange processes [228, 229]. The as-coated $\mathrm{ZnS}$ shell had a cubic structure and, as can be seen from Fig. 22(e), the substantial surface roughness of the ZnS indicated it was polycrystalline. This method could be modified and extended to the preparation of other semiconductor compounds that are also sensitive to oxygen, such as ZnSe and CdS [219]. 
In addition, wet chemically grown $\mathrm{ZnO}$ nanowires could also be used as a sacrificial template for the growth of other nanostructures, such as $\mathrm{ZnS}$ by vapor phase sulfidation via an ion exchange reaction [229], an $\mathrm{Al}_{2} \mathrm{O}_{3}$ thin layer by thermal annealing of an $\mathrm{AlCl}_{3}$ solution [221] or ALD [222], a $\mathrm{TiO}_{2}$ thin layer by sol-gel methods [222, 230, 231], and ZnS and ZnSe nanoparticles by wet chemical synthesis [232]. The $\mathrm{ZnO}$ core can subsequently be removed, and a tubular structure of such deposited layers can be prepared, as shown in Figs. 22(f) and 22(g).

\subsection{2 $\mathrm{ZnO}-$ metals}

Semiconductor-metal heterostructures exhibit many interesting chemical, optical, and electronic properties that have found various applications in catalysis, biomedicine, photonics, and optoelectronics [233]. In particular, noble metal nanoparticle-decorated semiconductor nanowires possess several merits. First is the surface enhanced Raman scattering effect due to a high density of hot spots on the surface of the semiconductor nanowires [234, 235]. Also, the decoration of $\mathrm{Ag}$, $\mathrm{Au}, \mathrm{Pt}$, or Co onto the $\mathrm{ZnO}$ nanowire surfaces changes the Fermi level equilibrium and band structure of the $\mathrm{ZnO}$ through storing and shuttling photogenerated electrons from the $\mathrm{ZnO}$ to acceptors in photocatalytic processes [137, 236, 237]. In addition, the photocatalytic efficiency is generally limited by the fast recombination of the photogenerated electron-hole pairs. But in the semiconductor-metal heterostructures, the photogenerated carriers will be trapped by the noble metal, which promotes interfacial charge-transfer processes and increases the carrier lifetime [238].

Pacholski et al. reported site-specific deposition of Ag nanoparticles onto $\mathrm{ZnO}$ nanorods by a photocatalytic wet chemical method, as shown in Fig. 23(a) [238]. The growth solution was composed of $\mathrm{AgNO}_{3}$ solution and well-dispersed $\mathrm{ZnO}$ nanorods. Under illumination by ultraviolet light, electrons and holes were separated in the $\mathrm{ZnO}$ nanorods. The electrons reacted with the absorbed $\mathrm{Ag}^{+}$ions reduced them to $\mathrm{Ag}$, and the holes concomitantly oxidized the alcohol molecules in the solution. The Ag nanoparticles grown by photoreduction were preferentially located at one end of the $\mathrm{ZnO}$ nanorods probably because of a preferentially small lattice mismatch between $\mathrm{Ag}$ and $\mathrm{ZnO}$ in the particular crystallographic planes as evidenced by HRTEM studies. Once an Ag nucleus was formed on the $\mathrm{ZnO}$ nanorod, it acts as a seed for the further photocatalytic reduction of $\mathrm{Ag}$, and thus more nuclei were prevented from forming on one $\mathrm{ZnO}$ nanorod. An inbuilt Schottky barrier was suggested at the $\mathrm{ZnO} / \mathrm{Ag}$ interface. Raman enhancement was observed in the Ag nanoparticles on $\mathrm{ZnO}$ nanowire arrays [92].

As the opposite process to the secondary growth of $\mathrm{Ag}$ nanoparticles on $\mathrm{ZnO}$ nanorods, Fan et al. demonstrated the secondary growth of $\mathrm{ZnO}$ nanorods onto the $\{111\}$ facets of $\mathrm{Ag}$ truncated nanocubes by a wet chemical method, as shown in Fig. 23(b) [239]. Using Ag nanocubes as the nano-substrate, $\mathrm{ZnO}$ selectively nucleated and grew on the eight $\{111\}$ facets. Due to spatial confinement, only seven branches of $\mathrm{ZnO}$ nanorods at most were observed. This growth mechanism was suggested to result from two factors. First is a low lattice and symmetry mismatch $(2.68 \%)$ between the $\mathrm{ZnO}(2 \overline{1} \overline{1} 0)$ spacing $(d=0.1625 \mathrm{~nm})$ and the $\mathrm{Ag}(\overline{1} 12)$ spacing $(d=0.16697 \mathrm{~nm})$. The other is the direct interface of the $\mathrm{Zn}$ layer with $\mathrm{Ag}$ that initiates the formation of the $\mathrm{ZnO}$ lattice. Convergent beam electron diffraction studies showed that $\mathrm{Zn}$ atoms were the first layer bonded to the surface of the Ag seeds and the growth front of the nanorod was an oxygen layer [239].

In addition to $\mathrm{Ag}$, $\mathrm{Au}$ nanoparticles have also been decorated onto $\mathrm{ZnO}$ nanowires by wet chemical methods. Au has excellent chemical stability, biocompatibility, and capability of near infrared excitation [240]. Liu et al. used a mixture of sodium citrate, ascorbic acid, and $\mathrm{HAuCl}_{4}$ reacted at room temperature for $5 \mathrm{~min}$ [130]. The as-synthesized Au nanoparticles were about $5-25 \mathrm{~nm}$ in diameter, and were well dispersed on the surface of the $\mathrm{ZnO}$ nanowires, as shown in Fig. 23(c). The Au-modified $\mathrm{ZnO}$ nanowires showed a distinct color change from the bare ones, which is consistent with a surface plasma resonance peak of $\mathrm{Au}$ at about $530 \mathrm{~nm}$. In addition to wet chemical syntheses, He et al. have described a novel approach to decorate $\mathrm{ZnO}$ nanowires with Au nanoparticles by electrophoretic deposition [241]. The basic working principle of electrophoretic deposition is that charged nanoparticles (in a colloidal solution) will be 

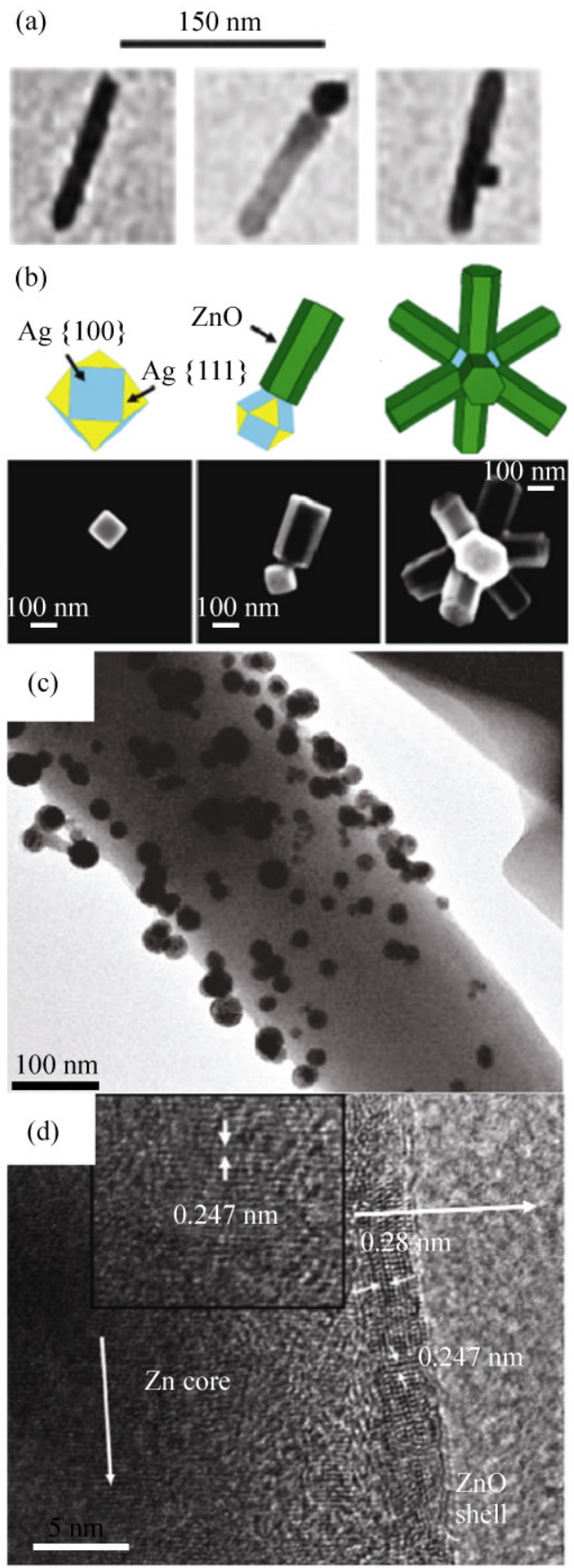

Figure 23 (a) TEM images showing $\mathrm{ZnO}$ nanorods with deposited Ag nanoparticles at different locations of the nanorod [238]. (b) SEM images showing the selective growth of $\mathrm{ZnO}$ nanowire branches on the $\{111\}$ facets of Ag truncated nanocubes, where the yellow and cyan planes represent the $\{111\}$ and $\{100\}$ facets of Ag, respectively; the green planes represent the facets of the $\mathrm{ZnO}$ nanowires [239]. (c) Low magnification TEM image of a Au-decorated $\mathrm{ZnO}$ nanowire [241]. (d) HRTEM image of a coaxial $\mathrm{Zn}-\mathrm{ZnO}$ core-shell nanorod [244]. Reproduced with permission driven to deposit on the target substrate under an external electric field. In their experiment, the gold colloidal solution was made by laser ablation of a gold target in water, which produced Au nanoparticles with fresh surfaces [242]. Then the $\mathrm{ZnO}$ nanorod array was immersed into the Au colloidal solution and used as the anode for the electrophoretic deposition. HRTEM studies revealed that the Au nanoparticles had good interfacial connection with the $\mathrm{ZnO}$ nanorods which is probably due to the strong van der Waals forces. Also, Au nanoparticles preferentially attach to the basal planes of $\mathrm{ZnO}$ since the interfacial energy between metal and polar surfaces is lower than that between metal and nonpolar surfaces [243]. Such Au-decorated $\mathrm{ZnO}$ nanowire arrays demonstrated very strong surface enhanced Raman spectroscopy activity due to the coupling effect between the Au nanoparticles and the $\mathrm{ZnO}$ nanowires [241]. In addition to post-treatment techniques, Shen et al. have reported a simple and mild solvothermal approach to co-precipitate a $\mathrm{ZnO}-\mathrm{Au}$ heterostructure by virtue of the low lattice mismatch between the $\mathrm{ZnO}$ (0001) planes and the $\mathrm{Au}$ \{111\} planes [137].

Besides $\mathrm{ZnO}-$ noble metal heterostructures, 1D Zn$\mathrm{ZnO}$ core-shell heterostructures have been obtained by a low temperature solvothermal approach [244]. As shown in Fig. 23(d), well-crystallized wurtzite $\mathrm{ZnO}$ was epitaxially grown along the [0100] direction, which is perpendicular to the [0002] direction along which the single crystalline $\mathrm{Zn}$ core was grown. The epitaxial relationship followed [0002]Zn/[0100]ZnO, and there were defects at the interface to accommodate the lattice mismatch [244].

\subsection{3 $\mathrm{ZnO}-$ carbon nanotubes}

Zhang et al. have demonstrated the fabrication of a $\mathrm{ZnO}$ nanowire/carbon nanotube heterostructure by hydrothermal synthesis, as shown in Fig. 24 [245]. In their method, a thin film of $\mathrm{ZnO}$ seed layer was precoated on vertically aligned carbon nanotubes by radio frequency (r.f.) sputtering or ALD [246]. After sputtering, the carbon nanotubes preserved their vertical alignment. The growth solution was made up of saturated $\mathrm{Zn}(\mathrm{OH})_{4}{ }^{2-}$ formed by dissolving $\mathrm{ZnO}$ in $\mathrm{NaOH}$ aqueous solution. The as-grown high density of $\mathrm{ZnO}$ nanowires on the carbon nanotube arrays

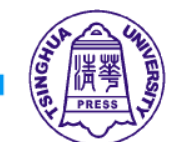

算 Springer 

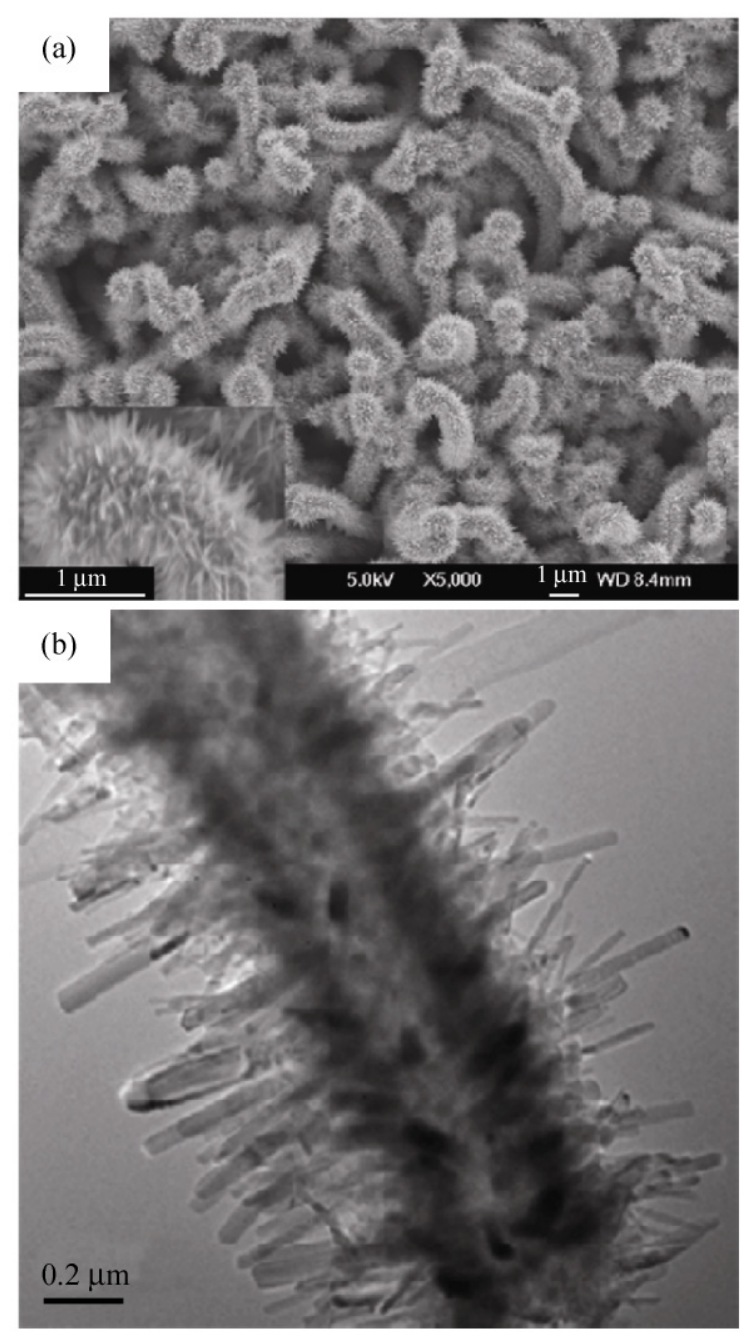

Figure 24 (a) Top view SEM image of $\mathrm{ZnO}$ nanowire-carbon nanotube heterostructures, and (b) TEM image showing the morphology of the radial $\mathrm{ZnO}$ nanowires on carbon nanotubes [245]. Reproduced with permission

showed greater surface to volume ratio than the $\mathrm{ZnO}$ nanowires grown on flat substrates. Moreover, the thin $\mathrm{ZnO}$ seed layer provided a continuous pathway for carrier transport for electronic applications [245, 247]. In another report [203], a thin layer of Al was coated onto carbon nanotube so that a hydrotalcite-like zinc aluminum hydroxide interfacial compound formed $[205,206]$, which was very effective in promoting the growth of $\mathrm{ZnO}$ nanorods.

\section{Rational doping and alloying}

Doping and alloying are the primary techniques to control the physical properties of semiconductor nano- materials, such as electrical conductivity, conductivity type, band gap, and ferromagnetism [86].

\section{1 -type doping}

$\mathrm{ZnO}$ nanowires are intrinsically $n$-type due to the inevitable point defects, such as oxygen vacancies and zinc interstitials. It is possible to substitute and thus reduce these defects by doping elements with similar electronegativities. Cui et al. used ammonium chloride to alter the growth properties of $\mathrm{ZnO}$ nanowires by electrochemical deposition [119]. They used an aqueous solution of $\mathrm{Zn}\left(\mathrm{NO}_{3}\right)_{2} \cdot 6 \mathrm{H}_{2} \mathrm{O}$, HMTA, and $\mathrm{NH}_{4} \mathrm{Cl}$ as the precursor. The successful doping of chloride into the $\mathrm{ZnO}$ nanowires was confirmed by compositional and structural analysis. The chloride-doped $\mathrm{ZnO}$ nanowires had larger diameters with reduced lengths. Photoluminescence studies demonstrated that the chlorine doping had two main effects. First was the reduced number of oxygen-related defects during $\mathrm{ZnO}$ growth. As shown in Fig. 25, the intensity of the visible broad band at around $530 \mathrm{~nm}$, originating from the emission of point defects, was gradually reduced as the concentration of the chloride ions in the precursor was increased, which indicates chloride helped to reduce the number of oxygen vacancies in $\mathrm{ZnO}$ nanowires. This phenomenon might be interpreted by considering the saturation of the growth surface by chloride, which limited the evolution of oxygen

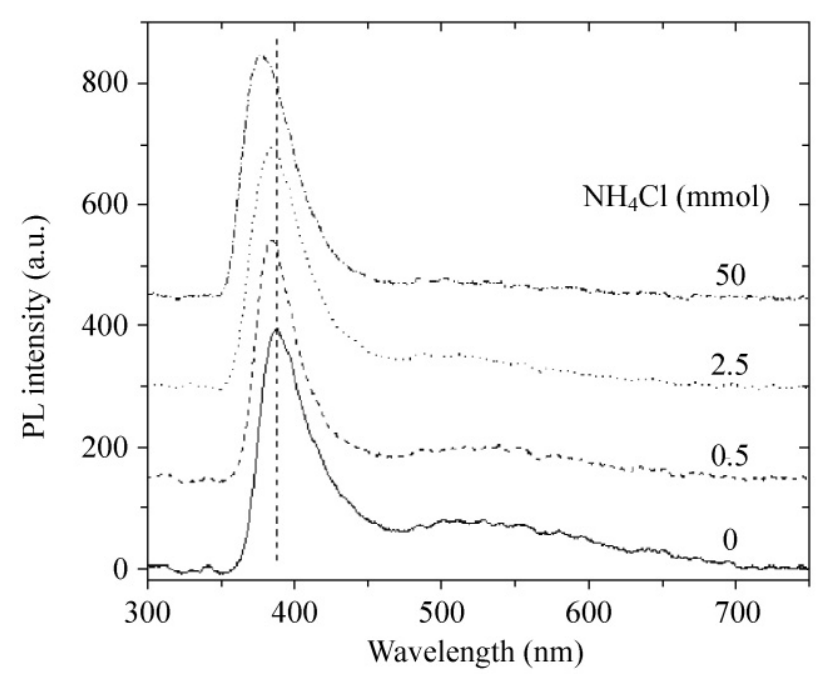

Figure 25 Photoluminescence spectra excited by a $248 \mathrm{~nm}$ laser of $\mathrm{ZnO}$ nanowires with different amount of chloride doping [119]. Reproduced with permission 
vacancies in $\mathrm{ZnO}$. Second, a blue-shift of $~ 10 \mathrm{~nm}$ of the band-edge emission peak at $385 \mathrm{~nm}$ was observed as the amount of chloride was raised from 0 to $50 \mathrm{mmol}$ in the growth solution, which indicates a widening of the band gap in $\mathrm{ZnO}$ nanowires, which was attributed to the blocking of the lowest states in the conduction band $[119,248]$.

Electrochemical deposition has been shown to be a powerful technique to dope $\mathrm{ZnO}$ nanowires. Beside chloride, Al-doped $\mathrm{ZnO}$ nanowire arrays have been fabricated by introducing $\mathrm{AlCl}_{3}$ [249], $\mathrm{Al}\left(\mathrm{NO}_{3}\right)_{3} \cdot 9 \mathrm{H}_{2} \mathrm{O}$ [250], or $\mathrm{Al}_{2} \mathrm{O}_{12} \mathrm{~S}_{3}$ [118] into the electrolyte. Microprobe analysis confirmed the incorporation of $\mathrm{Al}$ into the $\mathrm{ZnO}$ nanowires. The size of the $\mathrm{ZnO}$ nanowires could also be changed by tuning the $\mathrm{Al}^{3+}$ ion concentration in the electrolyte. After $\mathrm{Al}$ doping, it was found that the $\mathrm{ZnO}$ nanowires had higher electron mobilities and better conductivity in comparison with common undoped $\mathrm{ZnO}$ nanowires [118, 249], even though there was also a band gap widening after Al doping [119]. In addition, $\mathrm{Al}$ doping was also shown to have a effect on the mechanical and piezoelectric properties of $\mathrm{ZnO}$ nanowires as characterized by scanning probe microscopy [250].

\section{$4.2 p$-type doping}

It is highly desirable to make stable and reproducible p-type $\mathrm{ZnO}$ for fabricating $\mathrm{ZnO}$ homojunction optoelectronic devices, and this area still remains challenging and controversial [251]. Basically, to make p-type $\mathrm{ZnO}$ nanowires, group- $\mathrm{V}$ or group-I element atoms are needed to react and then diffuse through the defects of $\mathrm{ZnO}$ to replace oxygen or zinc atoms in $\mathrm{ZnO}$. There have been many efforts using both vapor phase [252, 253] and solution phase growth approaches [254]. For example, $p$-type doping of $\mathrm{ZnO}$ nanowire arrays has been reported by post-treatment of as-grown $n$-type $\mathrm{ZnO}$ nanowires, such as using $\mathrm{NH}_{3}$ plasma treatment [255], and thermal deposition and diffusion of As from GaAs wafers [256].

Utilizing a wet chemical method, Hsu et al. demonstrated intrinsic $p$-type $\mathrm{ZnO}$ nanowires [254]. In their report, $p$-type or $n$-type $\mathrm{ZnO}$ nanowires could be grown from the same growth solution at $90{ }^{\circ} \mathrm{C}$, and the key to controlling the conductivity type was found to be the preparation of the seed layer. $\mathrm{ZnO}$ nanowires fabricated on an electrodeposition seed layer exhibited $n$-type behavior, whilst those on zinc acetate derived seed layers showed $p$-type behavior. The difference in the conductivity type were attributed to several factors, such as the dependency of native defect concentrations, the different concentrations of zinc vacancies, and the different incorporation of compensating donor defects, like hydrogen and indium atoms. In their study, the as-grown $p$-type $\mathrm{ZnO}$ nanowires on the $n$-type seed layer homojunctions were characterized by many different techniques, such as measuring the current an capacitance dependence on the voltage and electrochemical impedance spectroscopy (EIS) measurements, as shown in Fig. 26. The presence of zinc vacancy related defects was confirmed by positron annihilation spectroscopy measurements.

The estimated hole concentration was on the order of $10^{17} \mathrm{~cm}^{-3}$ and was stable over a period of six weeks. More importantly, room temperature electroluminescence has been demonstrated based on homojunction and heterojunction light emitting diodes (LEDs) containing the as-grown $p$-type $\mathrm{ZnO}$ nanowires [254].

\subsection{Transition metal doping}

Transition metal doped dilute magnetic semiconductors are of particular research interest for potential applications in spintronic devices and visible light photocatalysis. A few studies have been reported of the synthesis and characterization of $\mathrm{ZnO}$ nanowires doped with different transition metal ions, like $\mathrm{Co}, \mathrm{Ni}$, $\mathrm{Mn}, \mathrm{Cu}, \mathrm{Fe}$, and Ag [86, 257, 258].

Simple addition of transition metal precursors into the $\mathrm{ZnO}$ nanowire growth solution does not necessary result in incorporation of transition metal atoms in $\mathrm{ZnO}$ nanowires. Cui et al. demonstrated a low temperature electrochemical deposition of $\mathrm{Co}$ and $\mathrm{Ni}$ doped $\mathrm{ZnO}$ nanowire arrays [120, 259]. In their recipe, cobalt or nickel nitrate was added to the conventional $\mathrm{ZnO}$ nanowire growth solution, under a negative potential of $0.8 \mathrm{~V}$ relative to a gold reference electrode. Quantitative energy-dispersive X-ray (EDX) spectral analysis showed concentrations of $1.7 \% \mathrm{Co}$ and $2.2 \%$ $\mathrm{Ni}$ in the nanowires. In contrast, when no potential was applied, there was no measurable doping by $\mathrm{Co}$ 

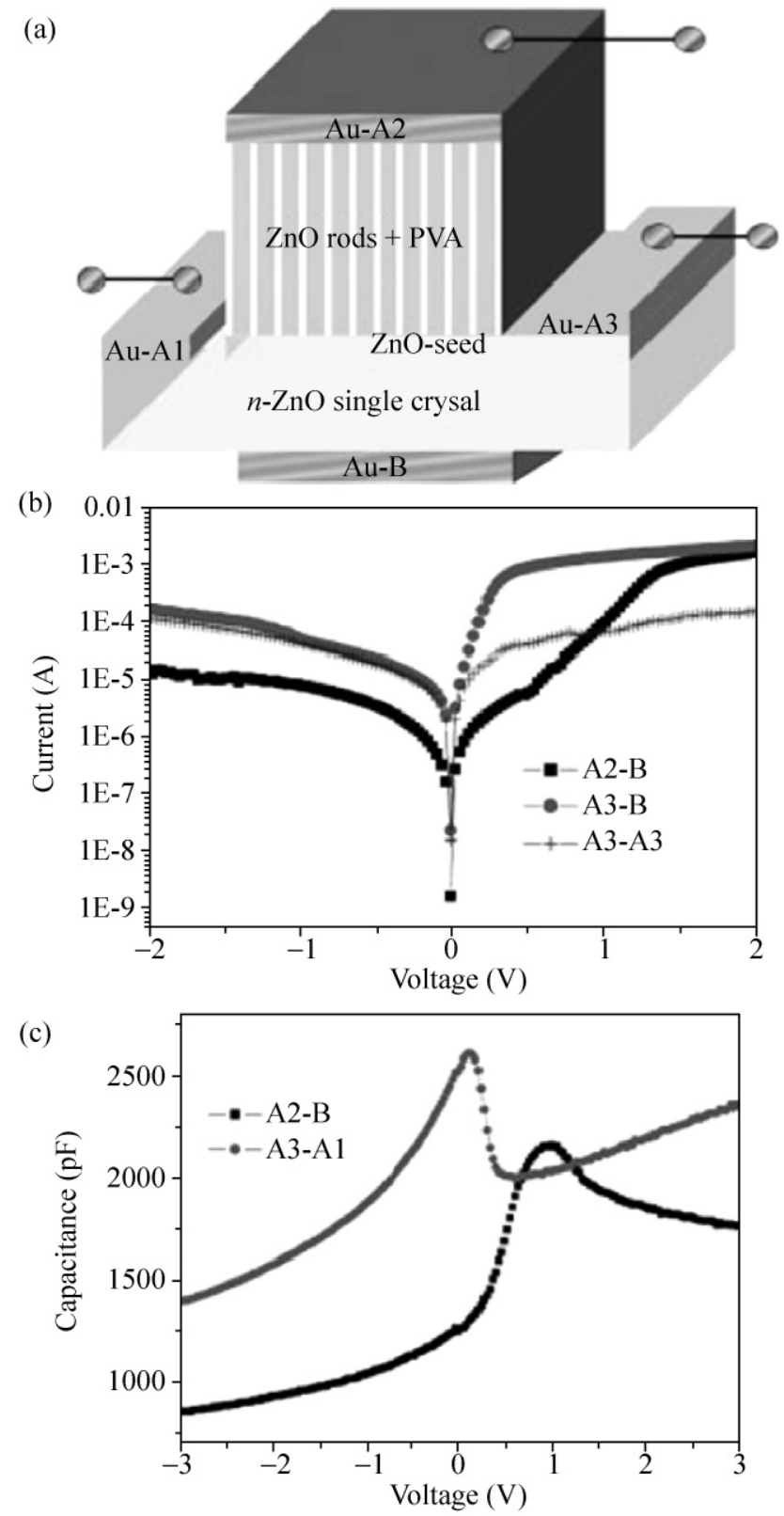

Figure 26 Capacitance-voltage measurements for $\mathrm{ZnO}$ nanowires grown with different seed layers. (a) Schematic device structure. (b) $I-V$ curves. (c) $C-V$ curves for the seed and rods [254]. Reproduced with permission

or Ni. Thus, the applied potential is critical, and it was suggested to adjust the interfacial energy and reduce the energy barrier for the incorporation of $\mathrm{Co}$ and $\mathrm{Ni}$ into the $\mathrm{ZnO}$ nanowires. XRD studies showed that after Co or Ni doping, the wurtzite structure of $\mathrm{ZnO}$ did not change, but the lattice expanded as evidenced from the shift in the (002) diffraction peak position. Room temperature anisotropic ferromag- netism was observed in both $\mathrm{Co}$ and $\mathrm{Ni}$-doped $\mathrm{ZnO}$ nanowire arrays, whereas undoped $\mathrm{ZnO}$ nanowires have paramagnetic behavior. As shown in Fig. 27, there was a hysteresis loop for the $\mathrm{Ni}$-doped $\mathrm{ZnO}$ nanowires when the magnetic field was applied parallel or perpendicular to the nanowire $c$ axis. The orientation of the easy magnetization axis could be parallel or perpendicular to the nanowire $c$ axis, and it was suggested to be dependent on the aspect ratio and density of the nanowires [120]. Annealing of the doped nanowires would help rearrange the dopants and enhance the magnetization. But when the annealing temperature was too high, it led to precipitation and clustering of the dopant atoms [260].

The Co-doped $\mathrm{ZnO}$ nanowires had interesting optical properties. When excited with ultraviolet light, both the near-band-edge emission and the defect emission peaks diminished in intensity after cobalt inclusion in the pristine $\mathrm{ZnO}$ nanowires. In addition, the peak centered at $680 \mathrm{~nm}$ in the photoluminescence spectrum corresponds to the transition from ${ }^{4} \mathrm{~T}_{1}(\mathrm{P})$ to ${ }^{4} \mathrm{~A}_{2}(\mathrm{~F})$ of tetrahedrally coordinated $\mathrm{Co}^{2+}$ ions in the $\mathrm{ZnO}$ lattice, which indicates the substitution of $\mathrm{Zn}^{2+}$ by $\mathrm{Co}^{2+}[258]$. The local dopant coordination environments were studied by X-ray diffraction and absorption spectroscopy [261]. The results showed that the transition metal doped $\mathrm{ZnO}$ nanowires were single crystal, single domain, and single phase in nature. The dopant ions were in a uniform environment which

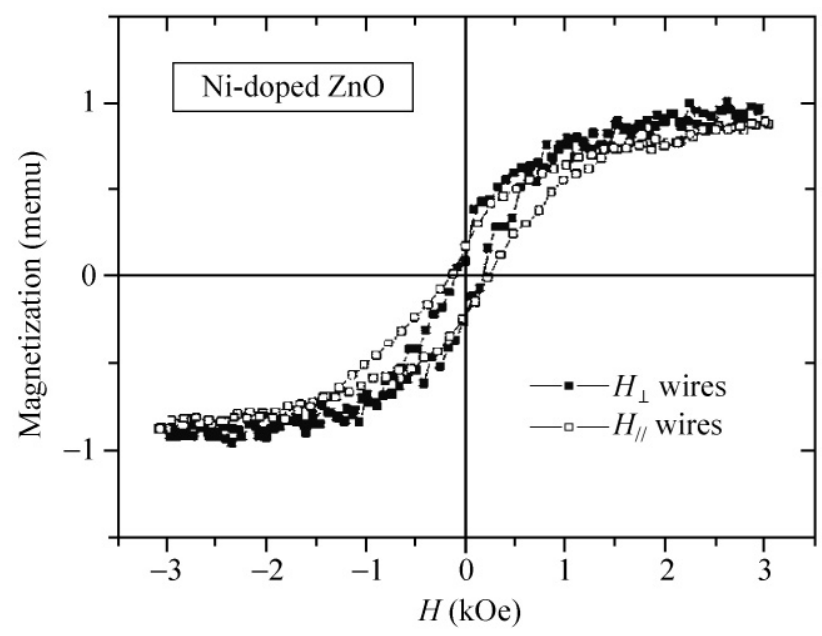

Figure 27 Magnetization hysteresis loop of Ni-doped $\mathrm{ZnO}$ nanowires with the applied magnetic field perpendicular and parallel to the nanowire $c$ axis [120]. Reproduced with permission 
did not induce a large degree of disorder in the nanowires. This homogeneous and uniform doping of the transition metal ions correlated with the weak ferromagnetic behavior of the nanowires [261]. Electron transport measurements on single Co-doped $\mathrm{ZnO}$ nanowires showed that the electron mobility could be as high as $75 \mathrm{~cm}^{2} /(\mathrm{V} \cdot \mathrm{s})$ [262]. Magnetotransport measurements showed that the magnetoresistivity was positive at low magnetic field due to the $s-d$ exchange induced spin splitting of the conduction band, and was negative at high magnetic field due to suppression of weak localization of impurity centers [262].

\subsection{Alloying}

Band gap engineering of $\mathrm{ZnO}$ by alloying $\mathrm{ZnO}\left(E_{\mathrm{g}}=\right.$ $3.37 \mathrm{eV}$ ) with $\mathrm{MgO}\left(E_{\mathrm{g}}=7.7 \mathrm{eV}\right)$ to form $\mathrm{Zn}_{1-x} \mathrm{Mg}_{x} \mathrm{O}$ alloys is an attractive approach for electronic and optoelectronic applications. Usually $\mathrm{ZnO}$ has a wurtzite hexagonal structure with a lattice parameter of $a=0.32$ and $c=0.52 \mathrm{~nm}$ (cubic $\mathrm{ZnO}$ is very rare), while $\mathrm{MgO}$ has a cubic structure $(a=0.42 \mathrm{~nm})$. Thus, alloying wurtzite $\mathrm{ZnO}$ with cubic $\mathrm{MgO}$ results in metastable wurtzite $(x>0.49)$ or zinc blende $(x<0.5)$ structures. Traditionally, $\mathrm{Zn}_{1-x} \mathrm{Mg}_{x} \mathrm{O}$ nanostructures are made using vapor phase approaches, such as metallorganic vapour phase epitaxy (MOVPE), metal oxide chemical vapor deposition (MOCVD), and PLD. Gayen et al. reported the growth of aligned $\mathrm{Zn}_{1-x} \mathrm{Mg}_{x} \mathrm{O}(0<x<0.2)$ nanowires by a wet chemical route on seeded glass substrates [263]. The aqueous solution was composed of $\mathrm{Zn}\left(\mathrm{NO}_{3}\right)_{2}$, $\mathrm{Mg}\left(\mathrm{NO}_{3}\right)_{2}$, and $\mathrm{NaOH}$. By assuming the percentage of $\mathrm{Mg}$ in the as-grown $\mathrm{Zn}_{1-x} \mathrm{Mg}_{x} \mathrm{O}$ nanowires was proportional to the percentage of $\mathrm{Mg}$ in the precursor [264], the results showed that changing the amount of $\mathrm{Mg}\left(\mathrm{NO}_{3}\right)_{2}$ relative to $\mathrm{Zn}\left(\mathrm{NO}_{3}\right)_{2}$ readily changed the value of $x$ in the $\mathrm{Zn}_{1-x} \mathrm{Mg}_{x} \mathrm{O}$ alloy. As shown in Fig. 28, on increasing the amount of $\mathrm{Mg}$ in the precursor solution, the aspect ratio of the alloyed nanowires increased significantly. XRD studies showed that the as-synthesized $\mathrm{Zn}_{1-x} \mathrm{Mg}_{x} \mathrm{O}$ nanowires were of single phase without phase separation. The value of $E_{\mathrm{g}}$ changed from 3.14 to $3.75 \mathrm{eV}$ as the $\mathrm{Mg}$ content was varied between 0.05 and 0.20 [263].

Shimpi et al. reported a two-step method for fabrication of uniform and large scale $\mathrm{ZnO}: \mathrm{MgO}$ nanowire arrays without post-annealing. In their method,
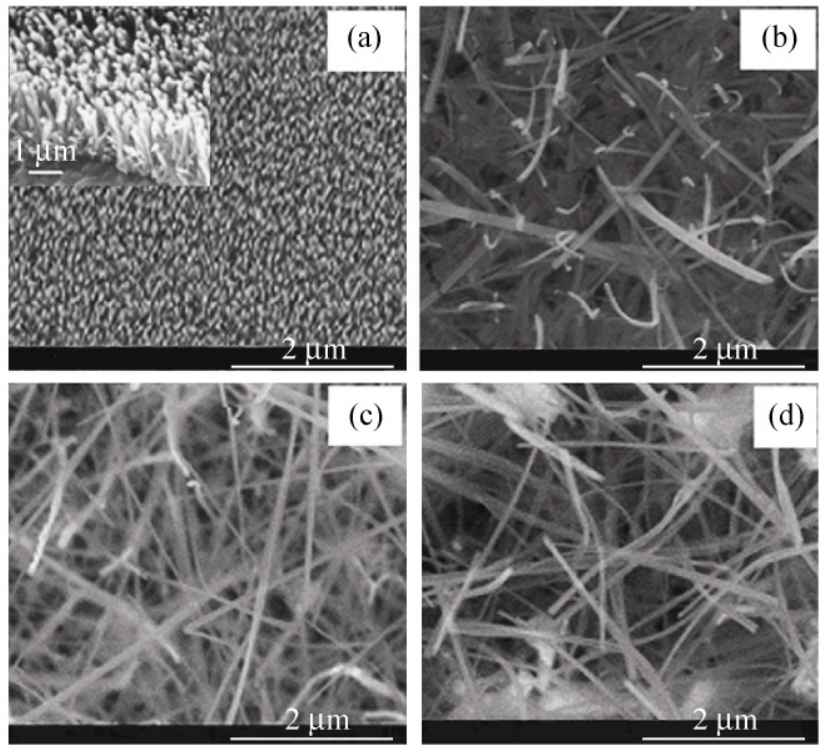

Figure 28 SEM images of $\mathrm{Zn}_{1-x} \mathrm{Mg}_{x} \mathrm{O}(0<x<0.2)$ nanowire arrays grown on seeded glass substrates with (a) $x=0$, (b) $x=0.05$, (c) $x=0.1$, and (d) $x=0.2$ [263]. Reproduced with permission

common $\mathrm{ZnO}$ nanowire arrays were immersed into an aqueous solution of $\mathrm{Zn}\left(\mathrm{NO}_{3}\right)_{2} \cdot 6 \mathrm{H}_{2} \mathrm{O}, \mathrm{HMTA}$, and $\mathrm{Mg}\left(\mathrm{NO}_{3}\right)_{2} \cdot 6 \mathrm{H}_{2} \mathrm{O}$ in a 1:1:2 ratio, at a growth temperature of $155{ }^{\circ} \mathrm{C}$ for $4 \mathrm{~h}$. In the photoluminescence spectra, $\mathrm{ZnO}: \mathrm{MgO}$ nanowires showed a blue-shifted near-band-edge UV emission at both room temperature and low temperature, relative to the pristine $\mathrm{ZnO}$ nanowires [265].

\section{Patterned growth}

It is interesting and essential to manipulate the building blocks into a regular form for future advanced nanodevices. Here we will discuss various strategies for defining the spatial distribution of $\mathrm{ZnO}$ nanowires on a substrate, including photolithography, electron beam lithography, interference lithography, nanosphere lithography, nanoimprint lithography, micro-contact printing, and inkjet printing. Finally we will also discuss the feasibility of pattern transfer.

\subsection{Photolithography}

Photolithography basically employs UV light to transfer a geometric pattern from a mask to photosensitive materials on the substrate [266]. In a typical process, first the substrate is spin-coated with a layer of

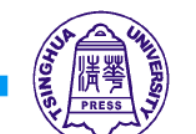

黑 Springer 
photosensitive material (e.g., photoresist), and then is exposed to UV light under a patterned photomask. The pattern on the photomask is usually generated by electron beam lithography, and the opaque area on the mask is covered by a metal, such as Cr. The subsequent step is to develop the exposed photoresist, which could be positive tone or negative tone. For a positive tone photoresist, high energy UV photons break up the chemical bonds and scissor the long polymer chains of the photoresist into short pieces. Therefore the area which has been exposed to UV light has a higher solubility in the developer than the area which has not. In contrast, for a negative tone photoresist, UV photons provide energy to overcome the energy barrier of forming new chemical bonds crosslinking the side chains of the small monomer molecules producing larger molecules that have a lower solubility in the developer than the original monomer molecules. In either case, the photoresist is selectively dissolved away and the local substrate underneath is exposed. In this way, a pattern is copied onto the substrate from a photomask that is reusable.

Tak et al. combined a photolithography procedure and a wet chemical method to grow patterned $\mathrm{ZnO}$ nanowire arrays [77]. The advantage of this technique is that the patterns can be made on a square inch scale in one batch, which results in high throughput and low cost. The wavelength of the UV light, however, is normally hundreds of nanometers, and therefore the feature size of the pattern is limited to the micrometer range, which gives rise to very dense nanowire arrays growing out of one spot on seeded substrates $[77,99]$. On a patterned substrate, the growth rate of the nanowires was shown to be inversely proportional to the nanowire density $[267,268]$, simply because the growth nutrient was shared between the competing nanowires. Also, the as-grown nanowires were not straight or uniformly oriented on seeded substrate [77], even though well-controlled $\mathrm{ZnO}$ nanowire arrays were grown on GaN substrates using photolithography with one or two nanowires growing out of one spot [269].

Photolithography could also be realized without a photoresist by using photosurface functionalization [270, 271]. Morin et al. demonstrated patterned growth of $\mathrm{ZnO}$ nanowire arrays on photosensitive polymers, such as commercial polycarbonate and polyester [271].
As shown in Fig. 29(a), the polycarbonate surface could be oxidized and grafted with carboxylic acid groups [272] or sulfate anion groups [273] where it was exposed to UV light in air, which was confirmed by fluorescence microscopy imaging [271]. The acidic groups could be further converted into hydroxyl groups by a simple hydrolysis [273]. The carboxylic acid groups generate a local acidic environment and thus suppress the nucleation and growth of $\mathrm{ZnO}$ nanowires. Without developing, the patterned polymer surface can directly be used to grow $\mathrm{ZnO}$ nanowire arrays by a wet chemical method. As shown in Fig. 29(b), wellorganized $\mathrm{ZnO}$ nanowire arrays were grown on the unexposed polymer surfaces [271].

(a)
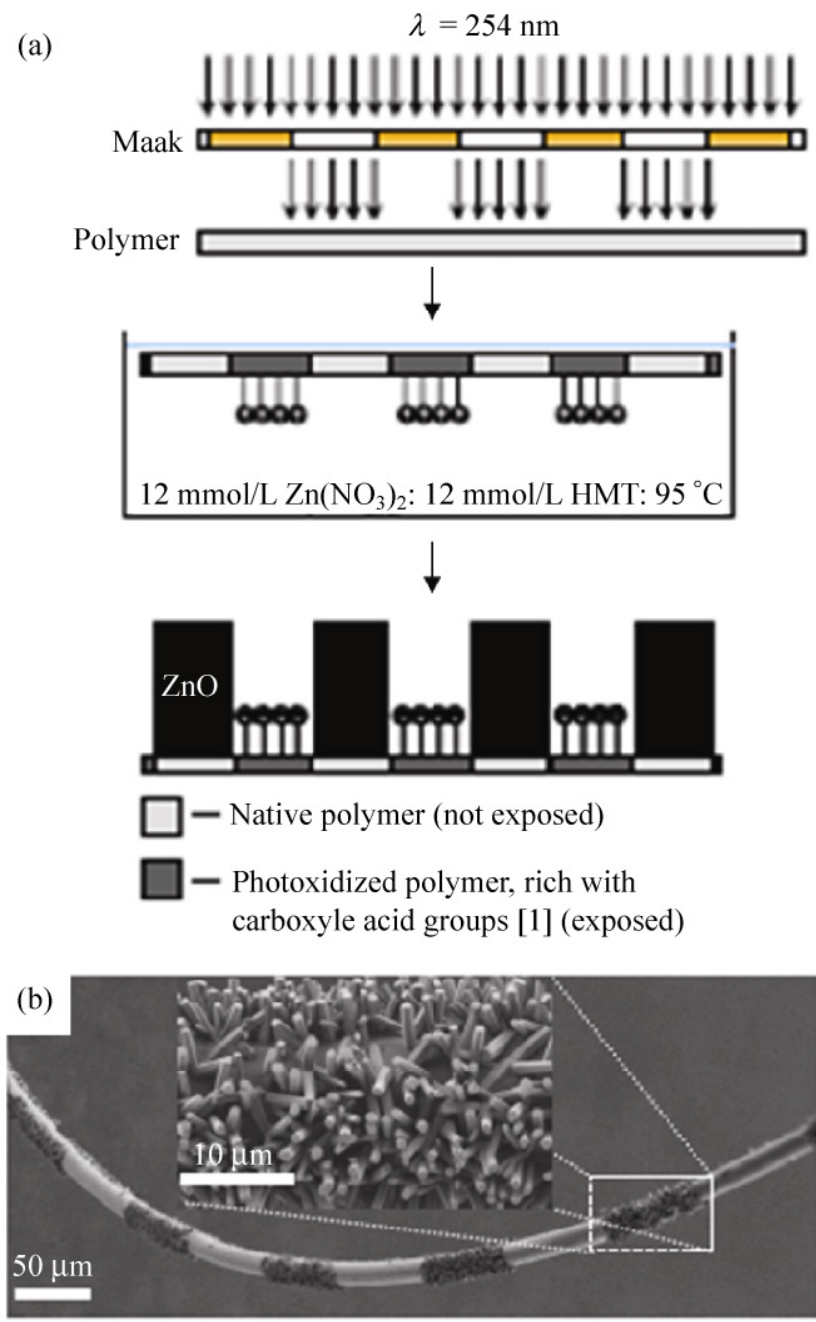

Figure 29 (a) Schematic flow chart of the use of UV oxidation to pattern a polycarbonate surface by photolithography, and (b) a SEM image of the as-grown $\mathrm{ZnO}$ nanowire arrays on the patterned polyester filament surface [271]. Reproduced with permission 


\subsection{Electron beam lithography}

Since photolithography has a bottleneck arising from the diffraction limit of UV light, a combination of electron beam lithography and a wet chemical growth method has been developed [48]. Electrons are accelerated by tens of kilovolts and have a wavelength on the order of Ångstroms, which gives a much higher resolution than conventional photolithography [274]. The growth mask can be a polymer resist, e.g., polymethyl methacrylate (PMMA), or any inert material, e.g., $\mathrm{SiO}_{2}$ [146].

The non-epitaxial growth of $\mathrm{ZnO}$ nanowire arrays on $\mathrm{Si}$ wafers (or any other inorganic/polymer substrates) can be assisted by a thin film of $\mathrm{ZnO}$ seed [48, 275]. Even though it had been annealed, the seed film was still composed of many tiny crystals several nanometers to tens of nanometers in size with random in-plane orientations. The opening size patterned by electron beam lithography was around $100 \mathrm{~nm}$, in which there were a number of seed grains exposed [48]. Each seed grain can give rise to one nanowire. Consequently in most cases, multiple nanowires grow out of one single patterned opening, as shown in Fig. 30(a). Interesting, by increasing the growth temperature from $70{ }^{\circ} \mathrm{C}$ to $95{ }^{\circ} \mathrm{C}$, the multiple nanowires from one single opening merge together and form a thicker nanowire (Fig. 30(b)), probably due to the fact that $\mathrm{ZnO}$ nanowires in close proximity are inclined to coalesce with each other [67, 72, 276].

Employing the epitaxial relation between $\mathrm{ZnO}$ and $\mathrm{GaN}$, well-aligned $\mathrm{ZnO}$ nanowire arrays have been realized, as shown in Figs. 30(c)-30(f) [48]. Single crystal $\mathrm{ZnO}$ wafers with or without metallic $\mathrm{Ru}$ thin film coating have also been used to grow well-aligned $\mathrm{ZnO}$ nanowire arrays [277]. The as-grown nanowires exhibited a remarkable vertical alignment and uniformity in diameter and length. By X-ray diffraction rocking curve measurements, $\theta-2 \theta$ scanning showed a full width at half maximum value of only $0.15^{\circ}$ [48]. It should be noted, that because the as-grown nanowires have a small diameter, a large aspect ratio, and possibly a weak connection to the substrate, the sample had to be dried in supercritical fluid to preserve the ordered alignment [278]. Otherwise, the residue solution droplets on the nanowires gradually evaporated away

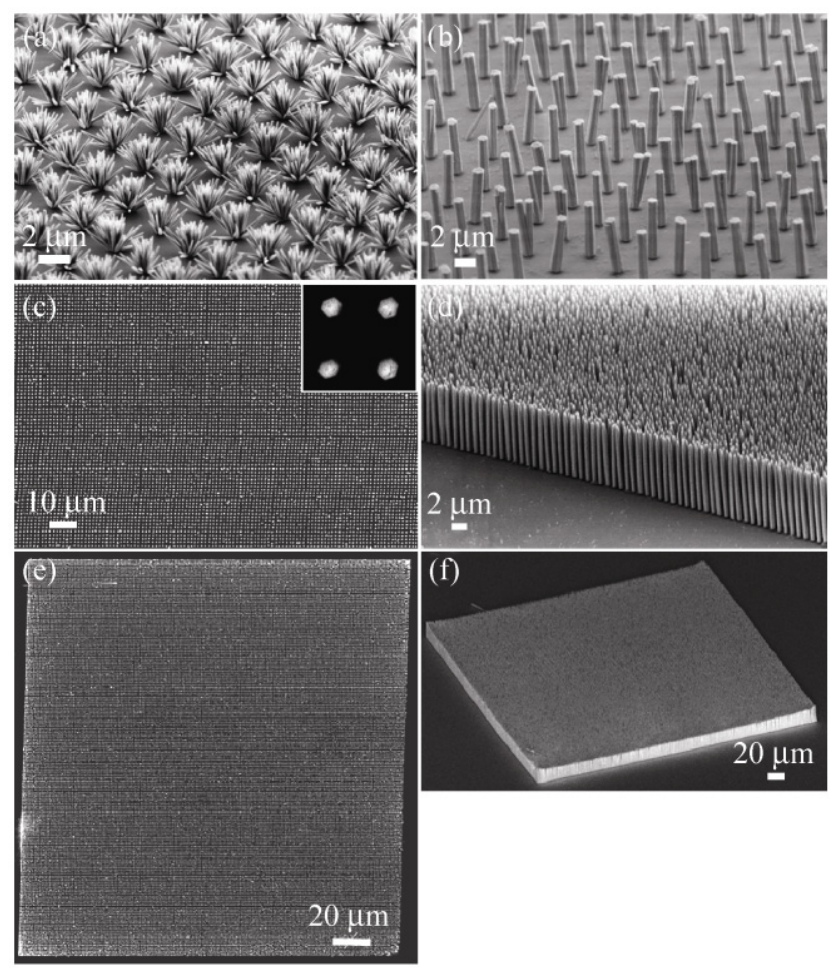

Figure 30 SEM images of the $\mathrm{ZnO}$ nanowire arrays on a seeded Si wafer grown at (a) $70{ }^{\circ} \mathrm{C}$ and (b) $95^{\circ} \mathrm{C}$. (c) Top view and (d) $60^{\circ}$ tilt view of the $\mathrm{ZnO}$ nanowire arrays on a $\mathrm{GaN}$ substrate grown at $95^{\circ} \mathrm{C}$. The inset in (c) is the enlarged view with a pitch of $1 \mu \mathrm{m}$. (e) Top view and (f) $60^{\circ}$ tilt view of a $200 \mu \mathrm{m} \times 200 \mu \mathrm{m}$ patterned $\mathrm{ZnO}$ nanowire array [48]. Reproduced with permission

and shrank in size, and the surface tension of the droplet bundled the nanowires together or swept them all down onto the substrate [279].

In physical vapor deposition, the width of the nanowire is often dictated by the size of the catalyst particles and normally does not change with growth time, simply because the incoming molecular species are preferentially adsorbed on the catalyst particles and the growth occurs at the interface between the catalyst particle and the solid nanowire. But in the wet chemical method, size expansion is always observed in both vertical and lateral directions when the nanowires are grown on pre-patterned substrates [48, 280]. The width of the as-grown nanowires was found to be almost three times the size of the patterned openings. Accordingly, a two-step growth process was proposed [48]. Firstly, the nanowire grew inside the patterned opening with the same lateral dimensions, as confined by the opening. As the nanowire grew out of the 
opening, there was no lateral confinement for the nanowire, and it can grow in both vertical and lateral directions, because incoming precursor molecules from the surrounding solution can adsorb on both top and side surfaces. But growth was apparently faster in the vertical direction than in the lateral direction as discussed previously. The growth in the lateral direction rendered the nanowire width greater than the opening size. Even though there was lateral expansion, the nanowire width could still be tuned by defining different opening sizes [48].

The main advantage of electron beam lithography is its high resolution. As can be seen from Figs. 30(e) and 30(f), a large array $(200 \mu \mathrm{m}$ by $200 \mu \mathrm{m})$ of welldefined $\mathrm{ZnO}$ nanowires, with a patterned opening diameter of $100 \mathrm{~nm}$ and $1 \mu \mathrm{m}$ in pitch, could readily be achieved [48]. Its major drawback, however, is the high cost and low throughput due to the long beamwriting time. In addition, the electron beam lithography system may not be stable, especially over large areas.

Horizontally aligned $\mathrm{ZnO}$ nanowire arrays have as many important applications as vertical ones [281]. But there has been only limited research and progress in this area. For example, a seed layer was put on the side wall instead of the top surface of the substrate to grow horizontal $\mathrm{ZnO}$ nanowires [282, 283]. There has also been a report of the epitaxial growth of horizontal nanowires by physical vapor deposition [284]. However, the uniformity and spatial control was rather poor. Horizontal alignment of the $\mathrm{ZnO}$ nanowires after growth was also achieved by dispersing the nanowires into solvents and then applying a high frequency alternating electrical field [285].

An unprecedented strategy for patterned horizontal $\mathrm{ZnO}$ nanowire arrays was demonstrated by combing electron beam lithography and a solution growth method [280]. The basic principle is illustrated in Fig. 31(a). For horizontal growth, $[2 \overline{1} \overline{1} 0]$ or [01 $\overline{1} 0]$ oriented single crystal $\mathrm{ZnO}$ substrates are required. Also, because of the anisotropic growth habits of the $\mathrm{ZnO}$ nanowires, the strip shape openings had to be along the [0001] direction of the substrate to grow individually separated nanowires. As shown in Fig. 31(b), the horizontal nanowires were epitaxially grown on the substrate with uniform length and width. Although the nanowires suffered from lateral expansion once they grew out of the mask confinement as discussed previously [286], the dimensions of the nanowires could still be adjusted by controlling the opening sizes. Also, to make use of the lateral expansion, multi-segment monolithic $\mathrm{ZnO}$ superstructures were demonstrated, as shown in Fig. 31(c) [56].

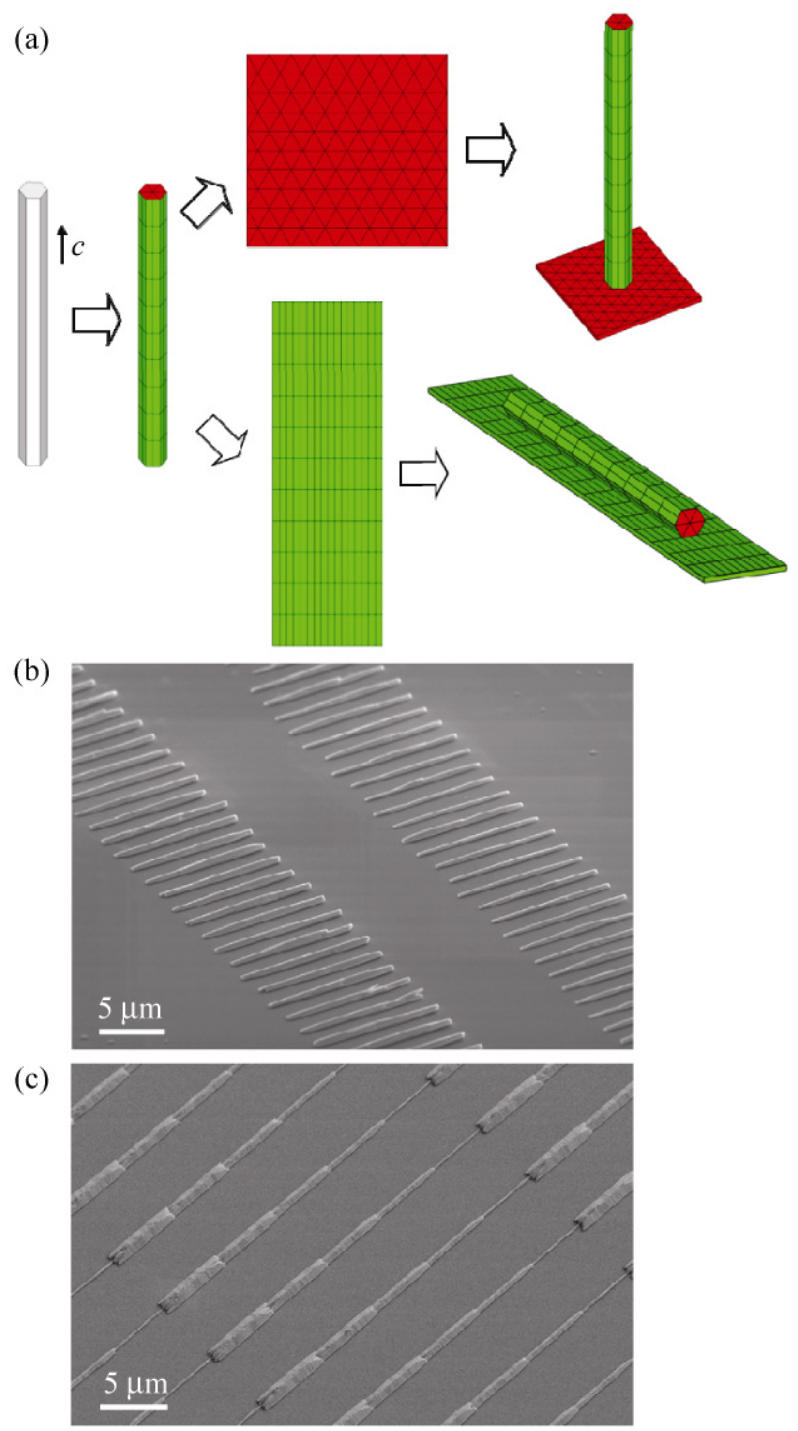

Figure 31 (a) From a crystal structure point of view, wurtzite structure $\mathrm{ZnO}$ has a six-fold symmetry at its basal surfaces and a two-fold symmetry at its side surfaces. On a six-fold symmetry epitaxial substrate, $\mathrm{ZnO}$ will grow vertically on the substrate. On a two-fold symmetry substrate, it will grow horizontally on the substrate. (b) A $60^{\circ}$ tilt view of thick (top row) and thin (bottom row) $\mathrm{ZnO}$ nanowire arrays growing out of $2 \mu \mathrm{m}$ by $400 \mathrm{~nm}$ and $2 \mu \mathrm{m}$ by $200 \mathrm{~nm}$ openings, respectively [280]. (c) A $30^{\circ}$ tilted view of the horizontal nanowire-array-based five-segment monolithic superstructures [56]. Reproduced with permission 
An electron beam could also be used to directly generate $\mathrm{ZnO}$ nanowires by writing on a zinc naphthenate resist [287]. The resist became insoluble in toluene after exposure to a high energy electron beam, giving rise to negative patterns. The as-fabricated polycrystalline $\mathrm{ZnO}$ nanowires could be reduced to sub-10 nm dimensions after calcination [287].

\subsection{Interference lithography}

Laser ablation was originally used to generate patterned $\mathrm{ZnO}$ seeds for the growth of $\mathrm{ZnO}$ nanowire arrays [288]. The laser is primarily used as a heating source to melt the precursor materials, which are later ejected through a mask. In addition to being a heating source, it was also found that a femtosecond laser was able to generate patterns on a single crystal $\mathrm{ZnO}$ wafer by virtue of the second harmonic generation excited in the $\mathrm{ZnO}$ wafer [289]. By changing the experimental conditions, different patterns such as dot arrays, ripples or regular gratings can be obtained. Other than that, based on laser interference, two-dimensional (2D) [290] or 3D [291] periodic structures could be generated by so-called laser interference lithography. The periodically distributed high intensity of light physically burned off the polymer mask layer $[292,293]$ or the substrate itself [294], which was used to pattern the growth of $\mathrm{ZnO}$ nanowire arrays. For example, in a study by Wei et al. [293], the nanowire growth method followed what had been previously reported [48, 91]. The benefits of laser interference lithography were claimed to be high throughput and low cost on a wafer scale, as shown in Fig. 32 [293].

\subsection{Nanosphere lithography}

Sub-micrometer sized spheres, such as polystyrene and silica spheres, will self-assemble on a water surface into a hexagonally close-packed structure driven by the water surface tension. A honeycomb pattern is formed [295], called nanosphere lithography. These spheres are commercially available with well-controlled monodisperse sizes. The hexagonally close-packed structure can be used as a mask, and the feature size is tunable by using spheres of different sizes and also by post-heat treatment [296]. When heated, the spheres deform and therefore the gaps between them shrink
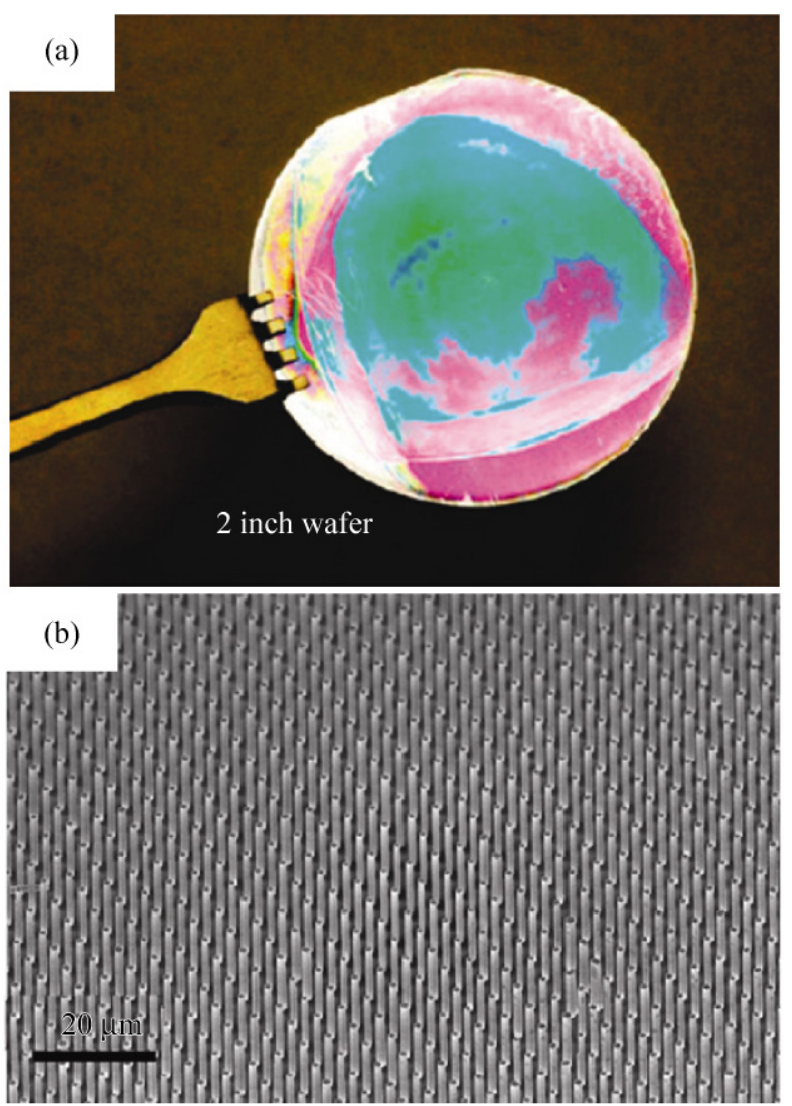

Figure 32 (a) Large scale growth of patterned $\mathrm{ZnO}$ nanowire arrays on a two-inch wafer by laser interference lithography, and (b) the corresponding SEM image [293]. Reproduced with permission

in size. Li et al. differentiated the connected pattern and the inverted pattern of the hexagonally close-packed spheres by introducing a secondary complementary replication process [297]. In Li's study, the growth of a single nanowire out of one gap was achieved by a wet chemical method, and the distribution of the nanowires had very good fidelity to the original sphere patterns. The aspect ratio of the nanowires was fairly small. Also, there were many defects in the self-assembled spheres. Nevertheless, nanosphere lithography offers a simple, cost-effective, and high throughput lithographical approach.

\subsection{Nanoimprint lithography}

Nanoimprint lithography is an embossing technique, which describes the transfer of a sub-micron scale pattern from the mold to the target substrate or device [298]. It is known for achieving sub-100 $\mathrm{nm}$ resolution on a large scale in a time-efficient and cost-effective

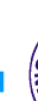

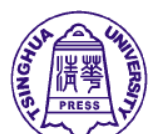

算 Springer 
manner. Basically, it employs a relatively hard mold template that is used to carve into a relatively soft surface. The hard molds are generally made of wearable and durable materials, such as silicon and flexible PDMS, which can be used repeatedly. The pattern in the mold is typically written by electron beam lithography. The mold surface properties can be tuned to be hydrophilic or hydrophobic [299]. An anti-stick coating is often applied on the mold to prevent any residues that will introduce defects into the pattern. Figure 33 shows a flow chart of the nanoimprint lithography process. The pattern is recorded on a piece of mold by electron beam lithography and chemical etching. On the substrate is a sol-gel derived $\mathrm{ZnO}$ seed layer. A mold is then embossed intimately into the seed layer. After drying, a complementary replica of the mold is made in the seed layer [300].

\subsection{Micro-contact printing}

With a similar methodology, micro-contact printing $(\mu \mathrm{CP})$ utilizes a mold inked with functional molecules [301]. When the mold is in contact with a substrate, the functional molecules are transferred and selfassemble on the substrate following the patterns of the mold, as shown in the Fig. 34(a). The self-assembled functional molecules can inhibit or promote the growth of $\mathrm{ZnO}$ nanowires locally. Hsu and coworkers developed an approach that combines $\mu \mathrm{CP}$ and selfassembled monolayers on a Ag surface to direct the

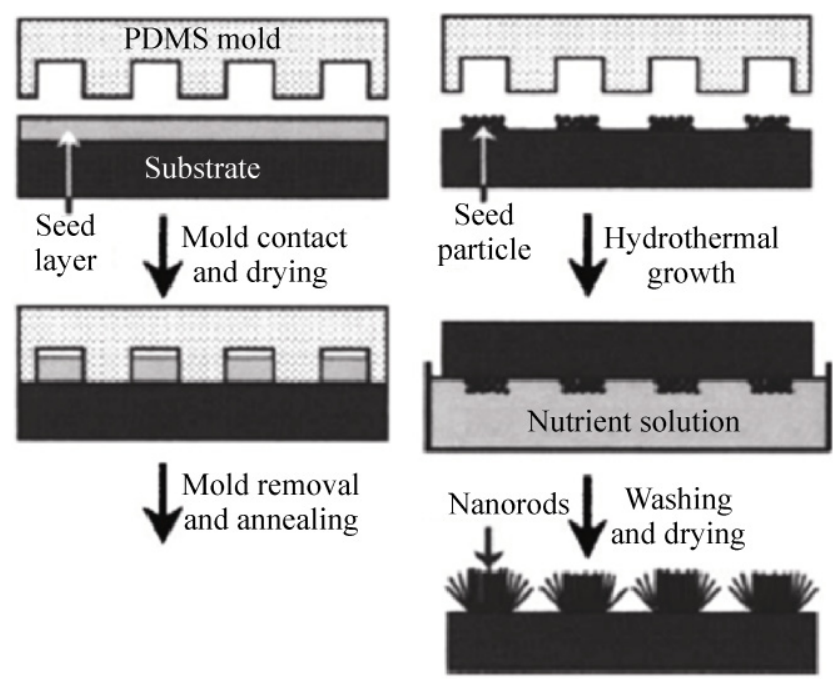

Figure 33 Schematic illustration of nanoimprint lithography [300]. Reproduced with permission

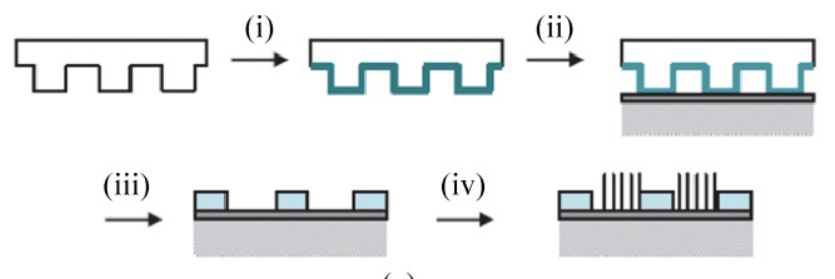

(a)

(i)

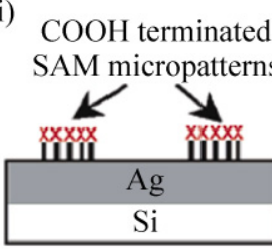

(ii)
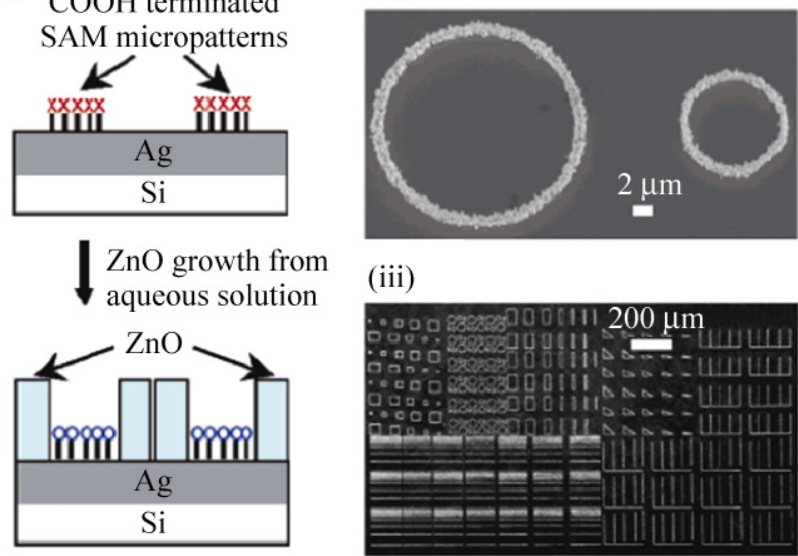

(iii)

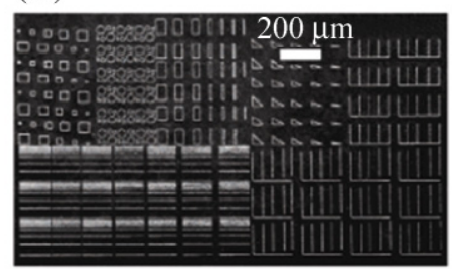

(b)

Figure 34 (a) Schematic illustration of micro-contact printing. (i) Inking the mold with octadecyltrichlorosilane (OTS) solution, (ii) $\mu \mathrm{CP}$ of the substrate, (iii) transferring the OTS pattern to the substrate, and (iv) $\mathrm{ZnO}$ growth on regions where there is no OTS [304]. (b) Patterned growth of $\mathrm{ZnO}$ nanowires on Ag with SAM patterns. (i) $\mu \mathrm{CP}$ patterned SAM on $\mathrm{Ag}$ surfaces (top) and the resulting $\mathrm{ZnO}$ nanowires on the bare $\mathrm{Ag}$ regions (bottom). $\mathrm{X}$ in the top panel denotes the $-\mathrm{COOH}$ end group; $\mathrm{O}$ in the bottom panel denotes $\mathrm{COO}^{-}-\mathrm{HMTA}-\mathrm{H}^{+}$complexes. SEM images of (ii) $\mathrm{ZnO}$ nanowires organized in two rings. The surrounding regions are covered by the SAM molecules, where no $\mathrm{ZnO}$ nanowires are grown, and (iii) large area patterns where $\mathrm{ZnO}$ nanowires appear white [302]. Reproduced with permission

spatial distribution of $\mathrm{ZnO}$ nanowire arrays [302]. In their particular case, they used a functional molecule that had a thiol group and a carboxyl group at its two ends. The thiol groups self-assembled and anchored themselves on the Ag surface; the acidic carboxyl group bound the HMTA molecules forming $\mathrm{COO}^{-}-\mathrm{HMTA}^{-} \mathrm{H}^{+}$ complexes which inhibited the local nucleation and growth of $\mathrm{ZnO}$ nanowires due to the electrostatic charge interactions between the charged species in solution and the surface-bound charged complexes. $\mathrm{ZnO}$ nanowires were observed to grow only on bare $\mathrm{Ag}$ surfaces, with more than one nanowire growing on each spot because the pattern feature size was much larger than the width of a single nanowire. Additives 
could be added to modify the growth behavior, and a thicker nanowire disk was formed on one spot [302]. The authors also found that the $\mathrm{COO}^{-}-\mathrm{HMTA}^{-} \mathrm{H}^{+}$ complexes played the major role in suppressing the nucleation of $\mathrm{ZnO}$. In their control experiment, when $\mathrm{Zn}^{2+}$ was first introduced to the carboxyl groups, the nucleation selectivity was much less obvious. Also, restricting regions of nucleation resulted in an increase in nucleation density in the unrestricted regions [303].

\subsection{Inkjet printing}

The basic structure of an inkjet printer is composed of a stage where the substrate is placed, at a distance away a nozzle from which ink is injected onto the substrate [305]. For the growth of $\mathrm{ZnO}$ nanowire arrays, a sol-gel derived zinc acetate precursor ejected from the nozzle can be dropped on a substrate that was heated to decompose the zinc acetate into $\mathrm{ZnO}$ seed crystals [306]. The typical resolution of an inkjet printed pattern is on the order of micrometers, and depends on many factors, such as the droplet size ejected from the nozzle, the accuracy of the droplet landing on the substrate, the wettability and spreading of the droplet on the substrate, and possibly convolution/interactions between the neighboring droplets. Of these factors, the size of the droplets is currently the bottleneck to narrowing down the feature size of this technique, and much effort is nowadays devoted to this topic [307], such as controlling the viscosity, evaporation rate, and surface tension of the precursor ink. Sekitani et al. have recently demonstrated an inkjet technology with micrometer line resolution [308]. In any case, inkjet printing provides a direct writing technique that is simple, versatile, and inexpensive and can potentially be scaled up.

\subsection{Pattern transfer}

The as-grown $\mathrm{ZnO}$ nanowire arrays can be transferred intact onto flexible substrates [56, 149]. Due to the lateral expansion of the $\mathrm{ZnO}$ nanowires from the patterned areas [48, 280], the actual contact area between the nanowire and the substrate is usually a fraction of the overall size of the nanowire, which enables easy cleavage at the interface without breaking the nanowires apart. In a typical pattern transfer process, a thin conformal layer of polymer was coated on the nanowires. In this process, selective adhesion of the coating polymer layer to the nanowires rather than to the underlying substrate is desirable, and possible interfacial bonds can be introduced between the coating polymer layer and the $\mathrm{ZnO}$ nanowires [56]. After that, another thicker layer of polymer was coated, which provided the flexible substrate with the mechanical strength to hold the nanowires. Then a straightforward peeling off or delamination was applied to the as-coated polymer layers. Rapid heating/cooling can also be used to separate the inorganic substrate and the polymer coating layers due to their different thermal expansion coefficients. Therefore, the patterned $\mathrm{ZnO}$ nanowire arrays can be transferred onto the as-coated polymer substrate, as shown in Fig. 35, which was later used to grow second generation of $\mathrm{ZnO}$ nanowire arrays, replicating the original pattern [193]. This technique has great potential for future flexible and foldable electronic applications [309].

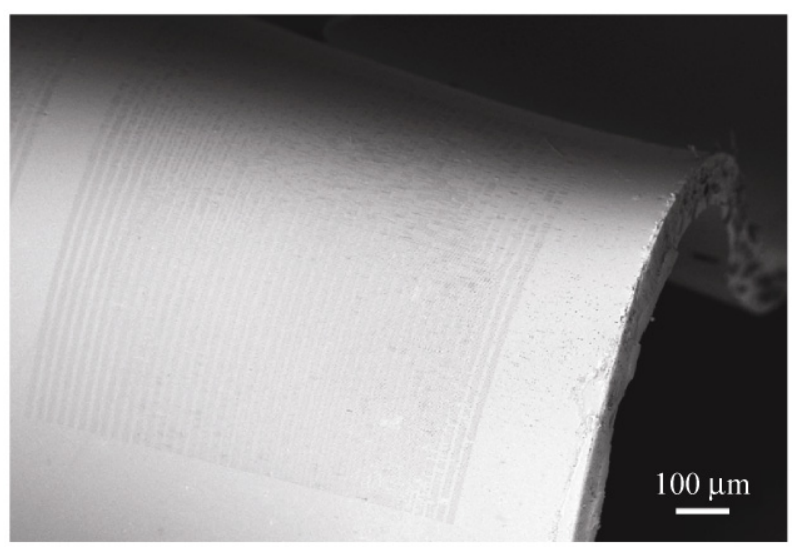

Figure 35 Low magnification SEM image of patterned nanowire arrays transferred onto a flexible substrate [56]. Reproduced with permission

\section{Properties and applications}

$\mathrm{ZnO}$ nanowire arrays have found innovative applications in a variety of areas due to their unique chemical and physical properties, as discussed specifically in the following sections.

\subsection{Catalytic properties}

$\mathrm{ZnO}$ has received great attention as a photocatalyst for the degradation and mineralization of environmental 
pollutants due to its large band gap and low fabrication cost [310]. Under UV illumination, $\mathrm{ZnO}$ will generate electron and hole pairs able to generate hydrogen by water splitting [311], synthesize $\mathrm{H}_{2} \mathrm{O}_{2}$ [312], and reduce graphene oxides to graphene [313]. In particular, the generated holes can be used to oxidize/decompose organic pollutants, such as rhodamine 6G [210], methyl orange [314], methylene blue [159], and formaldehyde [315]. Because of the presence of active defect sites - such as oxygen vacancies-on the surface, $\mathrm{ZnO}$ has also been used in industry as a catalyst for the synthesis of methanol from $\mathrm{CO}$ and $\mathrm{H}_{2}$ [316], or as a supporting scaffold for other catalysts, such as $\mathrm{Cu}$ [317], $\mathrm{Cu} / \mathrm{Fe}$ composites [318], $\mathrm{CuO}$ [319], $\mathrm{Au}$ [320], for oxidative steam reforming of methanol.

$\mathrm{ZnO}$ nanowires are an efficient photocatalyst due to their high surface to volume ratio in comparison with $\mathrm{ZnO}$ bulk materials. Zhou and Wong showed that $\mathrm{ZnO}$ nanowires had an even higher catalytic activity than nanoparticles and bulk forms due to their high purity and crystallinity [129]. In addition, if the size of the nanowire is smaller than a critical value of approximately $50 \mathrm{~nm}$, the effective band gap of $\mathrm{ZnO}$ will increase, the redox potentials will increase, and therefore the photogenerated electrons and holes will have a higher reducing/oxidizing power. In addition, with a larger band gap energy, the photogenerated electron and hole pairs will be less likely to recombine, which in turn enhances the charge transfer efficiency between the catalyst and the pollutants [310]. There are also other approaches to hinder the recombination of the photogenerated electron and hole pairs, such as using $\mathrm{CdS}$ nanoparticle- $\mathrm{ZnO}$ nanowire heterostructure arrays [321], and $\mathrm{SnO}_{2}$ and $\mathrm{ZnO}$ composites to improve the charge separation efficiency [322].

Figure 36(a) shows the absorption spectra of methyl orange solution with catalytic amounts of $\mathrm{ZnO}$ and different UV illumination times. The Beer-Lambert law states that the absorption is proportional to the concentration of the absorbing species. The intensity of the characteristic absorption peak at $464 \mathrm{~nm}$ for methyl orange decreases with increasing exposure time, and the peak completely fades away after about 80 min; as shown in the inset images of Fig. 36(a), the sample is also completely decolorized.

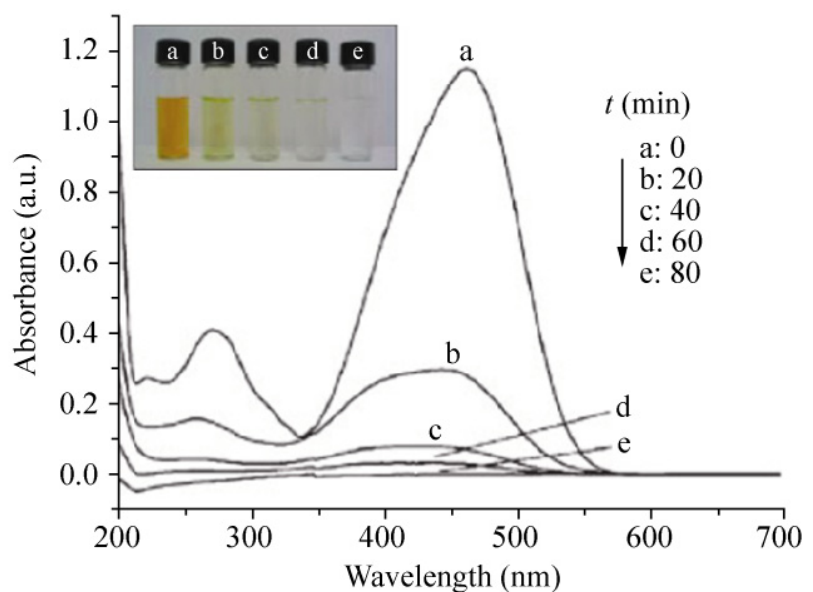

(a)

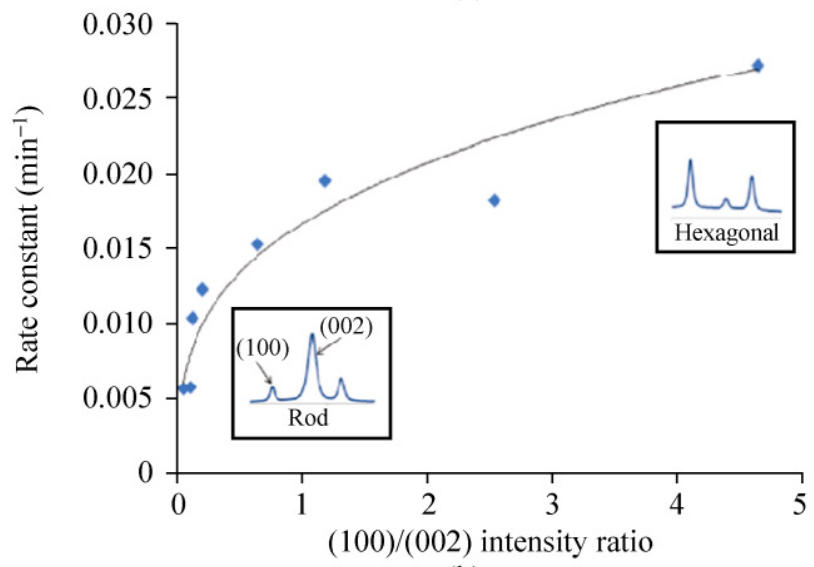

(b)

Figure 36 (a) Time-dependent color change (inset photo in the upper left) and corresponding optical absorbance spectra for methyl orange solution in the presence of micro/ nanoarchitectured $\mathrm{ZnO}$ after exposure to UV light [314]. (b) Plot of the photocatalytic decomposition rate constant as a function of the dominant facets (characterized by the XRD (100)/(002) intensity ratio) of the $\mathrm{ZnO}$ nanoparticles [159]. Reproduced with permission

It is widely accepted that catalytic properties are dependent on specific crystal facets, since different crystal facets have different dangling bond configurations [323]. Mclaren et al. carried out an interesting study showing that the polar (0001) or $(000 \overline{1})$ basal planes of $\mathrm{ZnO}$ have a stronger catalytic effect than the nonpolar $\{01 \overline{1} 0\}$ or $\{2 \overline{1} \overline{1} 0\}$ side planes for the photocatalytic decomposition of methylene blue [159]. In their study, they used hexagonal nanoplates and rod-shaped nanowires synthesized by controlling the oleic acid to zinc acetate ratio. As shown in Fig. 37(b), they found that on going from nanowires to nanorods and then to nanoplates, the ratio of the exposed basal 
planes to the side facets increased, and the photocatalytic decomposition rate constant of methylene blue increased accordingly. The activity of the nanoplates was more than five times higher than that of the nanowires. The higher activity of the polar basal planes was attributed to the intrinsically high surface energy of $\{0001\}$, which favors adsorption of the reactant molecules [159]. Li et al. attributed this high activity, however, to the correlation between the proportion of exposed polar surfaces and the surface oxygen vacancy density, since oxygen vacancies help to trap the photogenerated electrons and thus assist the charge separation [324].

The main disadvantage with $\mathrm{ZnO}$ photocatalysts is their stability and durability. $\mathrm{ZnO}$ is unstable in acidic and basic media, so photocorrosion under UV illumination is considered to be one of the main reasons for the decrease in $\mathrm{ZnO}$ photocatalytic activity over time. Therefore $\mathrm{ZnO}$ is only suitable for photocatalytic applications in neutral environments [314].

\subsection{Hydrophobic properties}

Wettability of a solid surface is of critical importance for many industrial applications [326]. Feng et al. reported reversible switching between superhydrophobicity (contact angle $>150^{\circ}$ ) and superhydrophilicity (contact angle $\sim 0^{\circ}$ ) of $\mathrm{ZnO}$ nanowire arrays by alternating $\mathrm{UV}$ irradiation and storage in the dark [325]. Figure 37(a) shows a water droplet on the $\mathrm{ZnO}$ nanowire array before (left image) and after UV illumination by a $\mathrm{Hg}$ lamp for $2 \mathrm{~h}$ (right image). The superhydrophobicity can be restored by putting the nanowires in dark for 7 days. This process can be repeated several times without obvious deterioration as shown in Fig. 37(b). This phenomenon was attributed to the correlation of the surface photosensitivity with the porous structure of the vertical $\mathrm{ZnO}$ nanowire arrays [327]. Wettability is governed by the material surface chemistry and also the geometrical structure of the surface [304, 328]. An as-grown $\mathrm{ZnO}$ nanowire array with appropriate density is a rather porous structure and exposes mostly the low energy non-polar side surfaces [101], which greatly enhances the hydrophobic behavior. Under UV irradiation, $\mathrm{ZnO}$ nanowires generate electron and hole pairs, and the holes can free the negatively charged

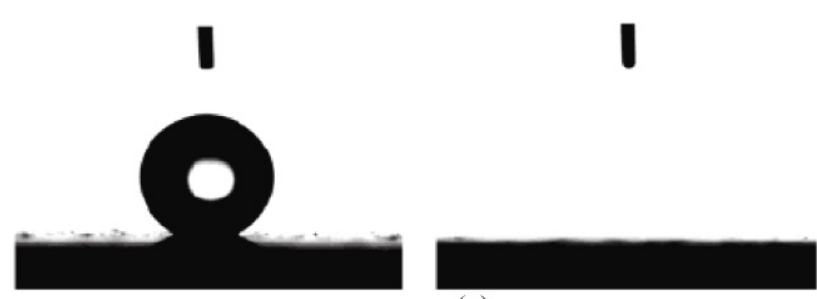

(a)

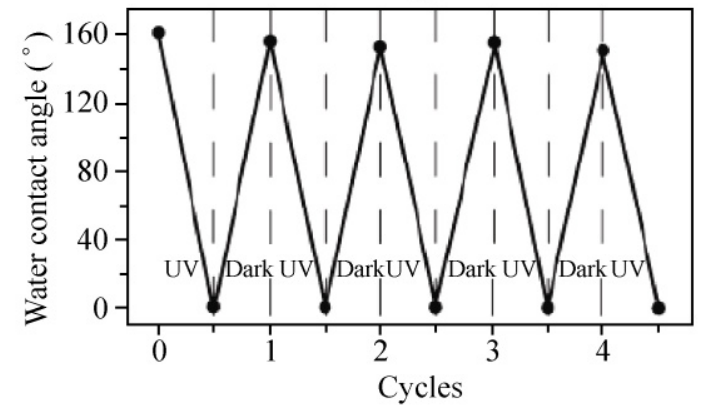

(b)

Figure 37 (a) Photographs of a spherical water droplet on aligned $\mathrm{ZnO}$ nanowire thin films before (left) and after (right) UV illumination. (b) Reversible superhydrophobic to superhydrophilic transitions of the as-prepared thin films under alternating UV irradiation and storage in the dark [325]. Reproduced with permission

oxygen molecules chemisorbed on the nanowire surfaces [329], leaving behind empty binding sites on the surface that are later occupied by hydroxyl groups, which therefore increases the hydrophilicity of the nanowire surfaces. Water droplets are sucked into the porous nanowire arrays by a capillary effect. In the dark, the hydroxyl groups on the $\mathrm{ZnO}$ nanowire surfaces are gradually replaced by oxygen molecules since their adsorption is actually thermodynamically more favorable [325].

As an alternative to UV irradiation, Badre and coworkers demonstrated an approach to reverse the surface wettability by controlling the electrochemical potential [330]. It has been shown that coating a monolayer of hydrophobic molecules on $\mathrm{ZnO}$ nanowire surfaces can considerably increase their hydrophobicity [331]. In Badre's study, they utilized a ferrocene silane molecule, N(3-trimethoxysilyl)propylferrocenecarboxamide, which has an anchoring group to $\mathrm{ZnO}$ nanowire surfaces on one end, and a redox change group on the other end. These two functional groups are separated by an alkyl chain. Under an electrochemical potential, the molecules can undergo ferrocene to ferrocenium redox changes, with ferrocene being the 
low free energy state and ferrocenium being the high free energy state. This change can be used to reversibly adjust the surface from hydrophobic to superhydrophilic. These smart surfaces could find applications in drug delivery and biosensors [331].

\subsection{Field emission properties}

Field emission finds applications in photoelectric panel display, X-ray sources, and microwave devices [332]. $\mathrm{ZnO}$, with the advantage of low manufacturing cost on a large scale and also allowing relatively high oxygen partial pressure during its operation, has become a good candidate for use in field emission cathodes.

It is desirable to have relatively high density and vertically aligned fine tips for enhanced local electric field around the tips [207, 333]. Hung et al. reported $\mathrm{ZnO}$ nanotip array-based field emitters [334]. The $\mathrm{ZnO}$ nanotip arrays (Fig. 38(a)) were grown by a hydrothermal method on a sputtered $\mathrm{ZnO}$ thin film or existing $\mathrm{ZnO}$ microrods, with a 1:2 ratio of zinc nitrate and HMTA as the nutrient. Field emission tests are usually carried out in vacuum at room temperature. The $\mathrm{ZnO}$ nanotip arrays are placed as the cold cathode, and a counter anode with known size is placed at a defined distance away from the cathode. An electric field is built up between the two electrodes when a voltage is applied [207]. The turn-on field and the threshold field are defined as the macroscopic fields required to produce a current density of $10 \mu \mathrm{A} / \mathrm{cm}^{2}$ and $10 \mathrm{~mA} / \mathrm{cm}^{2}$, respectively [112]. Figure 38(b) shows a plot of field emission current density as a function of the applied field [304]. The turn-on field can be effectively lowered by modifying the $\mathrm{ZnO}$ tips with metal nanoparticles [335]. Stability of the emission current density is usually a big concern in field emission technology [336].

The field emission behavior is governed by the so called Fowler-Nordheim (F-N) theory [337]: $J=$ $\left(A E^{2} \beta^{2} / \Phi\right) \exp \left(-B \Phi^{3 / 2} / \beta E\right)$, where $J$ is the emission current density in $\mathrm{A} / \mathrm{cm}^{2} ; E$ is the macroscopically applied electric field in $\mathrm{V} / \mathrm{cm}$, and $\beta$ is the field enhancement factor, defined by $E_{\text {local }}=\beta E=\beta V / d$, where $E_{\text {local }}$ is the local electric field around the emitter tips. $\beta$ reflects the degree of the field emission enhancement of a tip over a flat surface. $\beta$ is affected by many factors, such
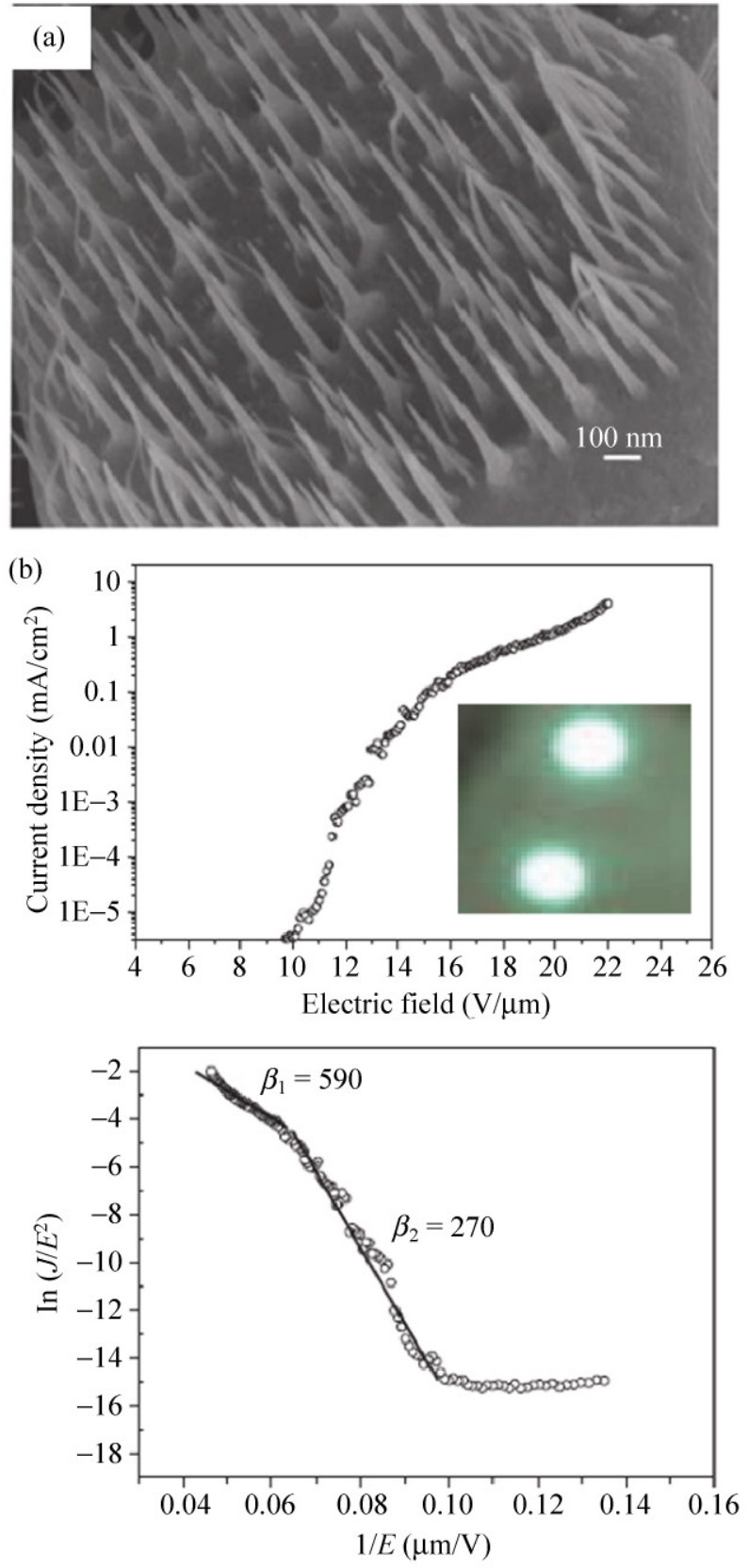

Figure 38 (a) As-grown $\mathrm{ZnO}$ nanotip arrays on existing $\mathrm{ZnO}$ microrods [334]. (b) Field emission current density as a function of applied electric field [334]. Inset is an uniform fluorescent field emission image on atypical green phosphor coated glass [339]. (c) The corresponding $F-N$ plot with different $\beta$ values indicated [334]. Reproduced with permission

as the emitter geometry, crystal structure quality, vertical alignment, and emitter density. It is desirable to have high $\beta$ values. Density of emitters plays an important role. If it is too low, then the emission current density will be low; if it is too high, then 
the local electric field around the emitter tips will have an electrostatic screening effect induced by the neighboring nanowires, which generally lowers the $\beta$ value $[112,338] . \Phi$ is the emitter work function, which is about $5.4 \mathrm{eV}$ for $\mathrm{ZnO}$ [337]. $A$ and $B$ are two constants with values of $1.56 \times 10^{10}\left(\mathrm{~A} \cdot \mathrm{V}^{-2} \cdot \mathrm{eV}\right)$ and $6.83 \times$ $10^{3}\left(\mathrm{~V} \cdot \mathrm{eV}^{-3 / 2} \cdot \mu \mathrm{m}^{-1}\right)$, respectively. By plotting $\ln \left(J / E^{2}\right)$ versus $1 / E$ as shown in Fig. 38(c) [334], the curve shows more than one slope, which may result from the variation in the tip local fields, or absorbate induced emission saturation [141]. When the applied field is high, the curve is roughly linear, which indicates the field emission current comes from barrier tunneling extracted by the applied electric field [75]. The $\beta$ values could be derived from fitting the experimental curve to $F-N$ theory according to $\beta=6.83 \times 10^{9} \mathrm{~d} \Phi^{3 / 2} / k$, where $d$ is the gap distance between the two electrodes and $k$ is the slope of the $F-N$ curve.

\subsection{Photonic crystals}

Periodically aligned vertical $\mathrm{ZnO}$ nanowire arrays give rise to a periodic modulation of dielectric constants for photons traveling inside, resulting in a refractive index contrast; this is called a photonic crystal [340]. Just as the periodic arrangement of atoms in crystals gives rise to a periodic distribution of electrical potential for electrons traveling inside, resulting in an electronic band gap, a photonic crystal has a photonic band gap for photons. Propagation of photons with frequencies within the photonic band gap is suppressed. Defects in the photonic crystal can also introduce localized states in the photonic band gap, allowing the propagation of photons with frequencies at the localized states. These properties of photonic crystals can be used to control the emission and propagation of photons for developing future integrated optical communications [341-345]. Inverted photonic crystals can also be made by infiltrating the empty spaces between the $\mathrm{ZnO}$ nanowires with polymers followed by dissolving the nanowires away with acids or bases.

Using an effective numerical method, as shown in Fig. 39(a), Kee et al. calculated the photonic band structures of a periodic array of vertically aligned $\mathrm{ZnO}$ nanowires with a frequency dispersion for the transverse magnetic (TM) mode (where the electric field is parallel to the $c$ axis of the nanowires) [340]. In their calculation, the periodic array of well-aligned nanowires can be treated as a $2 \mathrm{D}$ photonic crystal provided that the nanowire height is much larger than the nanowire width. Otherwise, if the nanowire height is too small, the $2 \mathrm{D}$ photonic crystal is too thin. In this case, its optical confinement is rather weak and it is not suitable for use as a waveguide. The calculation results showed that the center position and width of the band gap were dependent on the nanowire width and also the array pitch. By tuning the ratio between the two factors, a photonic band gap in the visible range could be obtained, as shown in Fig. 39(b) [340]. The crystal quality of the building blocks for the photonic crystals is very important, because defects such as voids and grain boundaries scatter electromagnetic waves leading to propagation loss. Figure 39(c) shows a typical transmission spectrum of a photonic crystal. The large full width at half maximum of the transmission peak indicates that it is a non-perfect photonic crystal [341, 342]. Figure 39(d) shows a six-fold optical diffraction pattern of white light passing through a hexagonally patterned $\mathrm{ZnO}$ nanowire array. Dispersion along the radial directions is clearly seen $[343,344]$.

\subsection{Light emitters}

ZnO-based light emitters have been considered as a potential candidate for the next generation of high efficiency blue/near-UV light sources, due to the direct wide band gap energy of $3.37 \mathrm{eV}$, a large exciton binding energy of $60 \mathrm{meV}$ at room temperature, and several other manufacturing advantages of $\mathrm{ZnO}$ [346], including its availability on a large scale at a relatively low cost, amenability to wet chemical etching, great tolerance to high energy radiation, and long-term stability. They can be used for a variety of technological purposes, such as solid state lighting, optical interconnects, and high density information storage.

\subsubsection{Optically excited light emission}

Using optical excitement of $\mathrm{ZnO}$ nanowires, researchers have studied their photoluminescence properties, realized lasing behavior from the Fabry-Perot cavity formed by the two basal planes, and also studied nonlinear effects. 


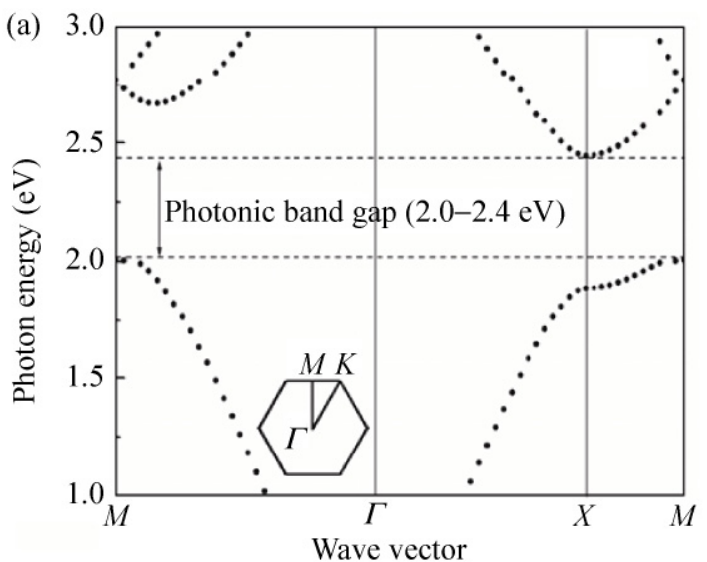

(c)
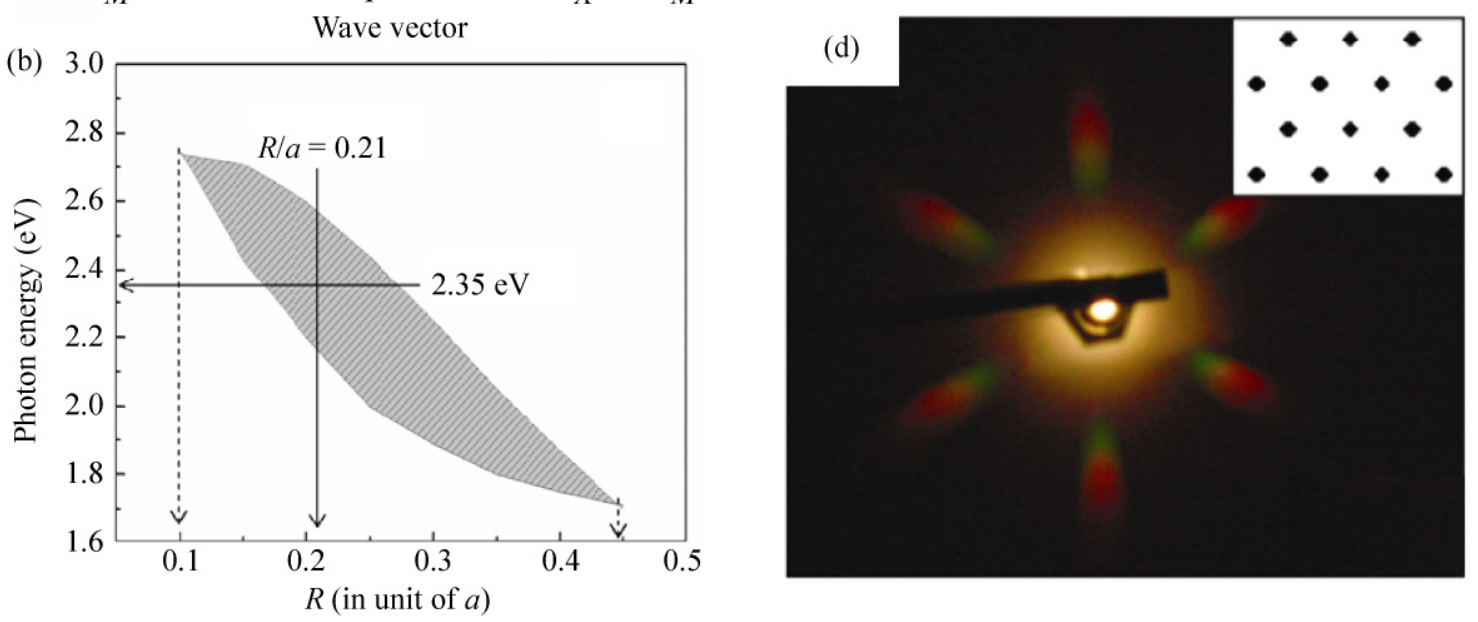

Figure 39 (a) The lowest three photonic bands for TM modes in a triangular lattice of $\mathrm{ZnO}$ nanowires when the array pitch $a$ is 240 $\mathrm{nm}$ and the nanowire width $2 R$ is $120 \mathrm{~nm}$, where $R$ is the nanowire radius. There is a photonic band gap in the range $2.0-2.4 \mathrm{eV}$. The inset denotes the first Brillouin zone of a 2D triangular lattice [340]. (b) The dependence of the band gap on $R / a$ when $a=240 \mathrm{~nm}$. The hatched area denotes the band gap. Note that when $R / a=0.21$, the photonic band gap center is located at around $2.35 \mathrm{eV}$, which is the spectral maximum frequency of the green luminescence band of $\mathrm{ZnO}$ nanowires due to oxygen vacancies or zinc interstitials [340]. (c) The measured transmission spectra of the $\mathrm{ZnO}$ nanowire hexagonal array in the $\Gamma-M$ direction for both TM and TE polarizations. The array has a photonic lattice constant of $250 \mathrm{~nm}$ and a height of about $500 \mathrm{~nm}$ [341, 342]. (d) Diffraction pattern using a white light source from a hexagonal array of $\mathrm{ZnO}$ nanowires [343, 344]. Reproduced with permission

\subsubsection{Photoluminescence}

The photoluminescence spectrum of $\mathrm{ZnO}$, excited by a UV laser at room temperature, usually has two emission bands. One is in the UV range, which is attributed to the near-band-edge emission through exciton-exciton collision processes [347]. The other is in the visible range, and presumably comes from the electron-hole recombination at a deep level in the band gap caused by intrinsic point defects and surface defects, e.g., oxygen vacancies, zinc interstitials, and the incorporation of hydroxyl groups in the crystal lattice during solution growth [124, 348-350]. These two emission bands have been used to enhance the photoluminescence intensity of rare earth compounds [351].

Figure 40(a) shows a typical photoluminescence spectrum of $\mathrm{ZnO}$ nanowires at room temperature [352]. The dominant UV emission peak is observed at around $380 \mathrm{~nm}$, together with a rather weak visible emission at about $500 \mathrm{~nm}$. Emission in the near-IR at around $760 \mathrm{~nm}$ for the $\mathrm{ZnO}$ nanowires grown on Au-coated ITO substrates is attributed to the second order feature of UV band-edge emission [353, 354]. The blue-shift in the peak position of the near-band-edge exciton emission as the size of the nanowire decreases to below $10 \mathrm{~nm}$ is possibly due to a confinement effect [355]. The defect-derived green emission usually results in 
a decrease in exciton lifetime and quantum efficiency of UV light devices, and therefore an increase in lasing emission threshold. To reduce the defect emission and to enhance the near-band-edge UV emission, post-plasma treatment, or annealing at different temperatures and in different atmospheres has been performed [356, 357]. Studies have shown that annealing at $200{ }^{\circ} \mathrm{C}$ can largely reduce the defect concentration as evidenced by positron annihilation spectroscopy [349]. A polymer coating on the $\mathrm{ZnO}$ nanowires was also demonstrated to effectively depress the defect emission and enhance the near-band-edge emission [358]. A photoluminescence spectrum of $\mathrm{ZnO}$ nanowires at $4.2 \mathrm{~K}$ is shown in Fig. 40(b) [338]. Generally as the temperature was decreased from room temperature to $4.2 \mathrm{~K}$, the photoluminescence intensity increased [104], and its temperature dependence

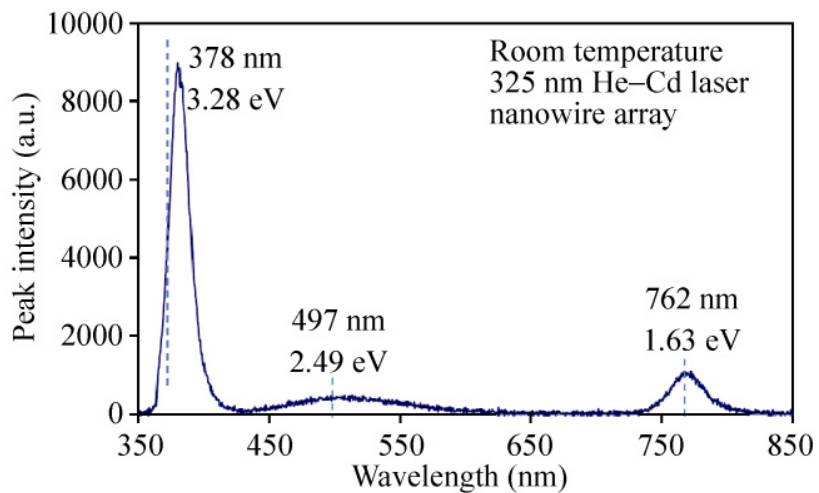

(a)

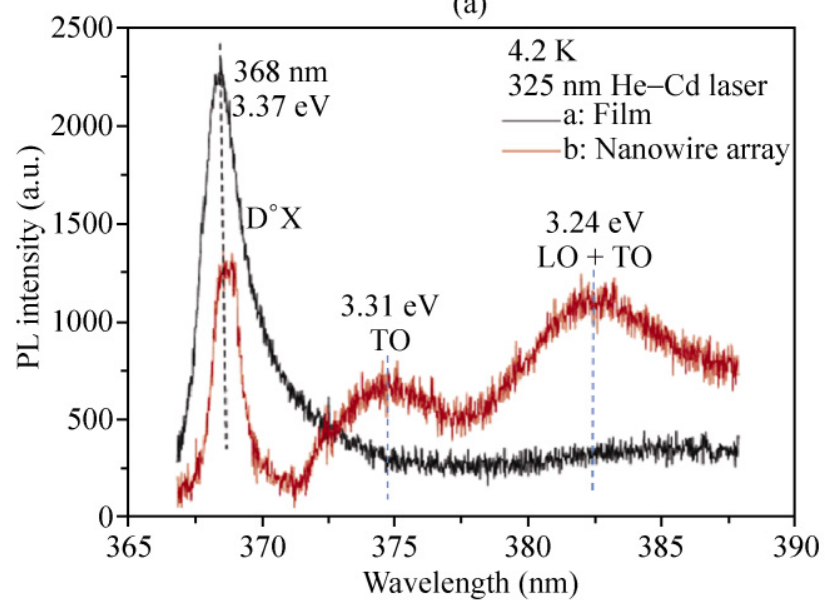

(b)

Figure 40 (a) Room temperature photoluminescence spectrum of $\mathrm{ZnO}$ nanowires grown on Au-coated ITO substrates at room temperature [352]. (b) Photoluminescence spectrum of $\mathrm{ZnO}$ nanowires and $\mathrm{ZnO}$ thin film at $4.2 \mathrm{~K}$ [338]. Reproduced with permission satisfied the Arrhenius relationship [184]. The main UV emission peak, assigned as the shallow bound exciton [338], was blue-shifted from $378 \mathrm{~nm}$ to $368 \mathrm{~nm}$, because the band gap of $\mathrm{ZnO}$ increases as temperature decreases following the Varshni relationship [359]. In addition, the longitudinal and transversal optical (LO and TO) phonon replicas were also apparent [338]. The energy separation between these phonon replicas, as labeled in Fig. 40(b), matched well with the reported phonon energies in $\mathrm{ZnO}$ crystals [1, 360-362].

\subsubsection{Optically pumped lasers}

$\mathrm{ZnO}$ nanowire arrays are good materials for the fabrication of room temperature lasers for two reasons [10]. First, $\mathrm{ZnO}$ is a wide band gap semiconductor with a large exciton binding energy $(60 \mathrm{meV})$ that is greater than the thermal energy at room temperature $(25 \mathrm{meV})$. So the excitons can be stable at room temperature, and efficient excitonic recombination is prerequisite for lasing. Second, the nanowire structure with two highly reflective top and bottom end facets forms a Fabry-Perot cavity, as shown in Fig. 41(a) [363]. Each nanowire has a Fabry-Perot cavity. Furthermore, the high refractive index of $\mathrm{ZnO}(n=2.45)$ can laterally confine the light traveling inside as a sub-wavelength waveguide for low order modes with an appreciable effective gain length.

Govender et al. demonstrated an optically pumped broadband visible laser based on $\mathrm{ZnO}$ nanowire arrays grown by a wet chemical method [10]. The nanowire arrays were optically pumped by a nitrogen laser, with the wavelength $(337 \mathrm{~nm})$ and pulse $(800 \mathrm{ps})$ matched to the band gap ( $365 \mathrm{~nm}$ ) and excitonic lifetime ( $350 \mathrm{ps})$ of $\mathrm{ZnO}$ nanowires. The pumping source was either in the vertical direction [10,363], or at a grazing angle to the substrate [152]. The detector was placed at the substrate normal. The threshold pumping power density $\left(I_{\mathrm{th}}\right)$ was determined by the slope change of the photoluminescence intensity $\left(I_{\mathrm{PL}}\right)$ as a function of the excitation laser intensity $\left(I_{\mathrm{ex}}\right)$. The $I_{\mathrm{th}}$ is thought to be sensitive to the density of states at the band edge of the material and the size of the cavity [9], and typically ranges from $40 \mathrm{~kW} / \mathrm{cm}^{2}$ to $7 \mathrm{MW} / \mathrm{cm}^{2}$ [152].

When the value of $I_{\mathrm{ex}}$ is less than $I_{\mathrm{th}}$, the emission is dominated by spontaneous emission; when $I_{\mathrm{ex}}$ is similar to $I_{\text {thr }}$ amplified spontaneous emission takes place; 


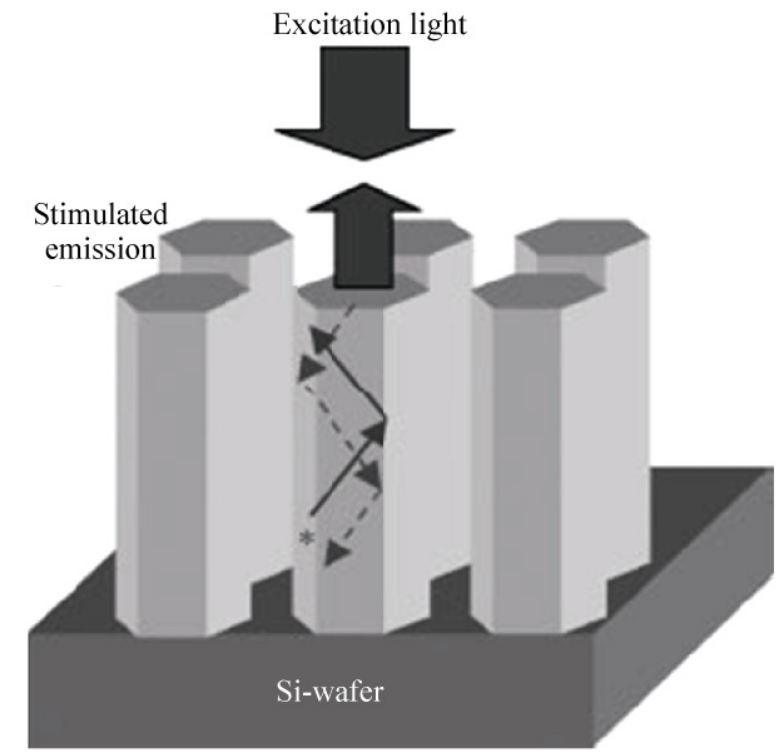

(a)

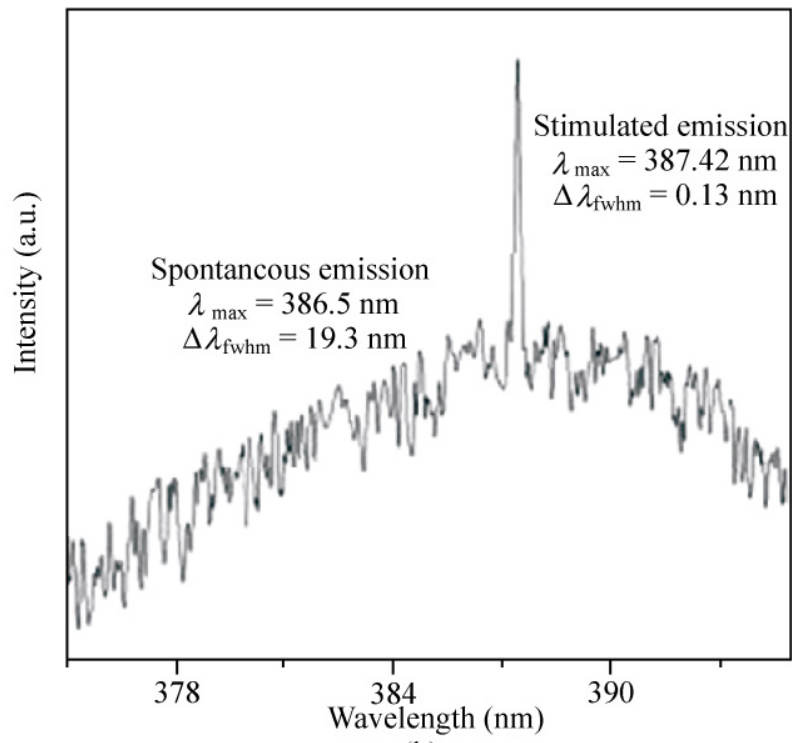

(b)

Figure 41 (a) Schematic illustration of the working principle of a nanowire Fabry-Perot cavity, in which the photons are bouncing back and forth between the two mirrors. (b) Emission spectrum of EHP lasing from $\mathrm{ZnO}$ nanowire arrays [363]. Reproduced with permission

when the value of $I_{\mathrm{ex}}$ is greater than $I_{\mathrm{th}}$ lasing shows up with a much narrower full width at half maximum (FWHM) than spontaneous emission, based on exciton and exciton scattering process; when the value of $I_{\mathrm{ex}}$ is further increased, a second stimulated emission due to the electron and hole plasma (EHP) can be observed. The EHP lasing peak is usually red-shifted in comparison with the exciton-based lasing, probably because of the band gap renormalization from the Coulomb interactions between the free electrons and holes at the band edge. The exciton lifetime generally decreases from spontaneous emission to EHP lasing [364].

The emitted photons oscillate between the two basal facets, and a series of emissions at the modal frequencies of the cavity can be detected. The wavelength spacing between these modes can be calculated by $\Delta \lambda=\lambda^{2} / 2 L(1 / n-\lambda(\partial n / \partial \lambda))$, where $n$ is the refractive index of the cavity, $L$ is the cavity length, and $\partial n / \partial \lambda$ is the frequency dispersion of the material [364]. The lasing modes covered almost the entire visible spectrum [10], and so can serve as a white light source. Choy et al. reported a second stimulated emission (EHP) peak at a wavelength of $387.42 \mathrm{~nm}$, as shown in Fig. 41(b) [363]. The FWHM was about $0.13 \mathrm{~nm}$, which was rather small. Because $\mathrm{ZnO}$ nanowires can serve as waveguides, random lasing through the side facets of the nanowires is not likely to happen [10]. Secondly, random lasing has a higher threshold than the lasing from the nanowire cavity $[10,364]$. Thirdly, random lasing is highly chaotic, while the lasing from the nanowire resonant cavity had a strong polarization in the horizontal substrate plane [364].

\subsubsection{Nonlinear optics}

Nonlinear optical properties of semiconductor nanowires have potential applications in frequency converters and logic/routing elements in optoelectronic nanocircuits [366]. In particular, $\mathrm{ZnO}$ with its polar surfaces can be used for frequency doubling of intense ultrashort laser pulses.

Voss et al. studied the resonant second harmonic generation (SHG) from $\mathrm{ZnO}$ nanowire arrays under excitation with intense femtosecond pulses [365]. In their experiment, they used a wavelength-tunable nearinfrared $(700-900 \mathrm{~nm})$ femtosecond oscillator to excite the nanowires. The light pulses had an energy of $12 \mathrm{~nJ}$ with $50 \mathrm{fs}$ duration. Figure 42 shows the emission curves for different excitation times. In the spectra, three different sets of peaks, in order of increasing wavelength, can be assigned as follows: the resonant SHG at a wavelength of $360 \mathrm{~nm}$ for femtosecond oscillator excitation of $720 \mathrm{~nm}$, the near-band-edge photoluminescence, and the emission from deep defect 


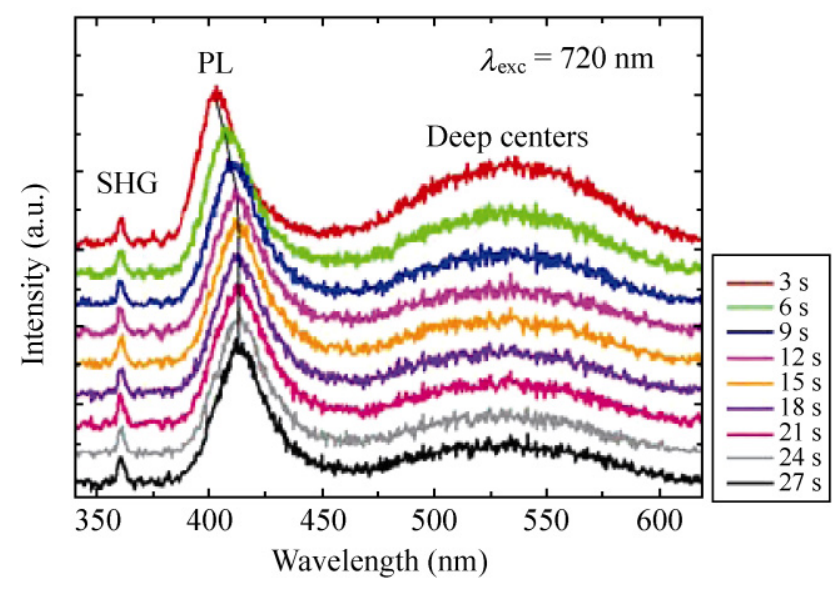

Figure 42 Emission spectra of $\mathrm{ZnO}$ nanowire arrays at different time delays after the excitation laser was unblocked. The integration time was $100 \mathrm{~ms}$ for all curves [365]. Reproduced with permission

centers. The photoluminescence peaks exhibited a red-shift during the first $27 \mathrm{~s}$ after the intense laser excitation, because of the band gap shrinkage induced by the temperature rise in the nanowires. Both $s-$ (TE) and $p$ - (TM) polarized excitation pulses were used to selectively detect the $p$-polarization component of the nonlinear optical output. A phenomenological four-layer model was used to simulate the angular dependence of the polarization curve. The ratio between the two relevant components of the $\left.\chi^{(2)}\right)_{11} / \chi^{(2)_{333}}$ was found to be -0.1 . The effective length of the nanowires was calculated to be $2.3 \mu \mathrm{m}$, compared with $3.2 \mu \mathrm{m}$ obtained from SEM images [365].

\subsubsection{Electrically driven light emission}

Light emission driven by electricity is more attractive than that driven by optical pumping, in the sense that the device is capable of being integrated with chip circuits. At present, electrically driven light emission from wet chemically grown $\mathrm{ZnO}$ nanowires is limited to LEDs. Electrically pumped lasers are difficult to realize due to the low crystal quality of the $\mathrm{ZnO}$ nanowires which leads to a low internal quantum efficiency and thus a high threshold voltage. The device usually fails before reaching the lasing threshold voltage due to Joule heating at high bias voltages.

\subsubsection{ZnO homojunction LEDs}

The most efficient solid state light emitters are based on $p-n$ junctions [251]. Hsu et al. demonstrated the hydrothermal synthesis of undoped $p-\mathrm{ZnO}$ nanowire arrays, and used as-grown $p$-type $\mathrm{ZnO}$ nanowire arrays to fabricate homojunction LEDs [254]. Figure 43 shows the photoluminescence and electroluminescence spectra of the $p$ - $\mathrm{ZnO}$ nanowires on $\mathrm{A} n-\mathrm{ZnO}$ single crystal substrate. When under bias, a blue peak and a broad red/near infrared emission band could be observed. Inset is a camera image of the LED when under bias. The blue peak arises from the exciton recombination of electrons from substrate and holes from the nanowire, and the broad red/near infrared emission is likely to be due to electroplex emission since this band was absent in the photoluminescence spectra [254].

\subsubsection{Inorganic heterojunction LEDs}

More often in LEDs, instead of $\mathrm{ZnO}$ homojunctions, heterojunctions of $n$ - $\mathrm{ZnO}$ and other $p$-type materials have been used, of which $p$-GaN is of particular interest considering it has similar crystallographic and electronic properties to $\mathrm{ZnO}$ [11, 367-369]. In addition, it has been suggested that the $\mathrm{ZnO}$ nanowire/GaN substrate heterojunction has a higher carrier injection efficiency and a higher recombination rate than other junctions [370].

In all of the previous cases, the $n-\mathrm{ZnO}$ nanowires were randomly distributed on the substrate. However, $\mathrm{Xu}$ et al. have demonstrated the possibility of controlling the spatial distribution of blue/near-UV LEDs

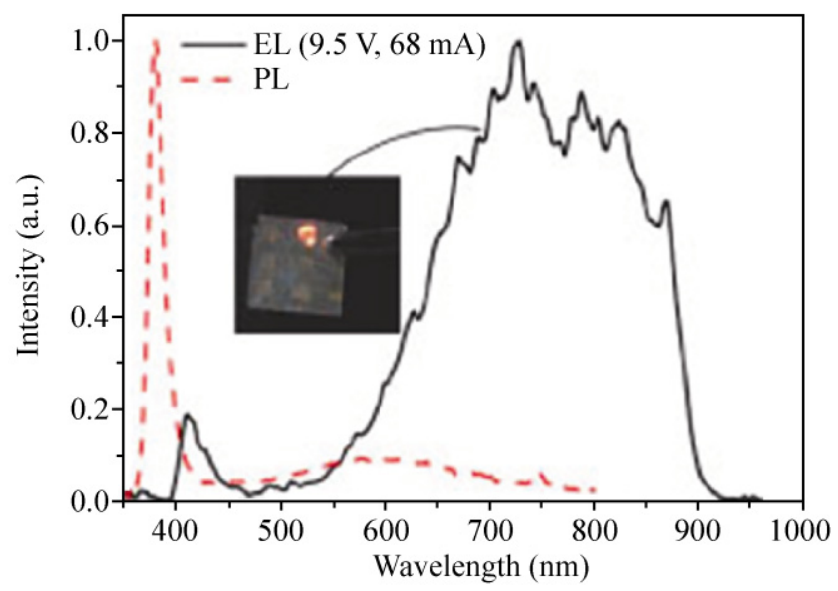

Figure 43 Photoluminescence (red dashed curve) and electroluminescence (black solid curve) spectra for a $p$ - $\mathrm{ZnO}$ nanowire array grown on a $n-\mathrm{ZnO}$ single crystal substrate. Spin-on-glass was used as an insulating layer to fill up the space between the nanowires. The inset image shows the LED when lit up [254]. Reproduced with permission 
based on position-controlled arrays of $n$ - $\mathrm{ZnO}$ nanowires on a $p-\mathrm{GaN}$ thin film substrate [371]. The device was fabricated by combining a low temperature wet chemical method and electron beam lithography [48]. Ohmic contacts to the bottom $p$-GaN and top $n-\mathrm{ZnO}$ nanowires were made by $\mathrm{Ni} / \mathrm{Au}$ and ITO films, respectively. The need for deposition of an ohmic electrode on the GaN substrate could be avoided by employing a $\mathrm{ZnO}$ nanowire/GaN substrate/ $\mathrm{ZnO}$ nanowire heterostructure [370]. The turn-on voltage of the diode was about $3.5 \mathrm{~V}$, which is relatively low, probably due to the presence of interfacial defects [372] owing to the low growth temperature $\left(<100{ }^{\circ} \mathrm{C}\right)$ of the chemical method. Figure 44 shows an optical image (exposed for $9 \mathrm{~s}$ with a gain value of 1 ) of the lit-up LED at a forward bias voltage of $10 \mathrm{~V}$. In the device, all of the nanowires were connected in parallel and each single nanowire is a light emitter. The difference in brightness of the individual nanowires probably arises from the different serial contact resistances with the bottom GaN substrate and top ITO electrode, which leads to different injection currents.

The physical origin of the electroluminescence has been extensively studied but is still under debate [373375]. There has also been a controversy about whether the emission is from the $n-\mathrm{ZnO}$ side $[147,376]$ or the $p$-GaN side [372, 377]. In reality, the hole concentration in the $\mathrm{Mg}$ doped $p$-GaN substrate $\left(\sim 10^{18} \mathrm{~cm}^{-3}\right)$ is higher than the electron concentration in the $n-\mathrm{ZnO}$ nanowire $\left(\sim 10^{17} \mathrm{~cm}^{-3}\right)$, but the carrier mobility in the $p-\mathrm{GaN}$ is

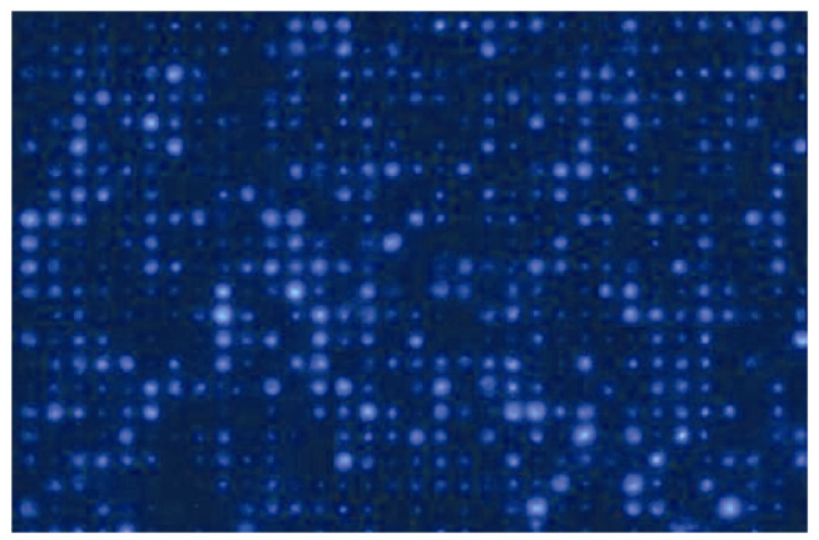

Figure 44 Optical image with artificial bluish color of a turnedon LED from ordered $\mathrm{ZnO}$ nanowire arrays grown on $p$-GaN thin film. The pitch is $4 \mu \mathrm{m}$ and the resolution is 6350 dpi. [371]. Reproduced with permission smaller than in the $n$ - $\mathrm{ZnO}$. Also the barrier heights at the interface for the holes $(0.57 \mathrm{eV})$ and for the electrons $(0.59 \mathrm{eV})$ are comparable. In this case, the charge injection by electrons and holes should be comparable from both sides. Of course, the energy band offset at the $\mathrm{ZnO} / \mathrm{GaN}$ interface is largely determined by the fabrication process [378], and any possible interfacial absorbates incorporated during the wet chemical growth [379].

As shown in Fig. 45(a), the emission spectra of the $n$-ZnO nanowire/ $p$-GaN thin film LED were monitored at different bias voltages at room temperature. From $4 \mathrm{~V}$ to $10 \mathrm{~V}$, the dominant emission peak was slightly blue-shifted in the range $400-420 \mathrm{~nm}$ with a FWHM of about $60 \mathrm{~nm}$. Peak deconvolution of the blue/near-UV emission with Gaussian functions (shown in the inset in Fig. 45(a)) showed that the broad blue/near-UV emission spectra consisted of three distinct bands (Fig. 45(b)) centered at 395-415 nm, 420-440 nm, and 450-510 nm. Each emission band corresponded to a particular recombination process [374]. The near-UV emission band centered at around $400 \mathrm{~nm}$ was attributed to the near-band-edge emission in $\mathrm{ZnO}$ nanowires originating from the recombination of $\mathrm{ZnO}$ free and bound excitons [377, 380]. Whereas the redshifted violet emission band centered at about $430 \mathrm{~nm}$ was ascribed to the transitions from the conduction band or shallow donors to deep $\mathrm{Mg}$ acceptor levels in the $p-\mathrm{GaN}$ thin film substrate $[372,377,380]$. The blue emission at around $460 \mathrm{~nm}$ was assigned to the radiative interfacial recombination of the electrons from $n-\mathrm{ZnO}$ and holes from $p$-GaN [380-382]. The interface states generally act as nonradiative centers that annihilate free electrons and holes. Therefore, a high quality interface is required in order to obtain high efficiency LEDs. The weak red emission at around $790 \mathrm{~nm}$ comes from the native deep level point defects (oxygen vacancies and zinc interstitials) in $\mathrm{ZnO}$ nanowires [377]. It should be noted that the emission spectra could be changed by thermal annealing [380].

As the bias voltage was increased, the intensities of the main peak and of all of the four sub-bands increased exponentially and the bands were blueshifted. The blue-shift has been attributed to several different factors, such as band renormalization and band filling [383], and the screening effect of the built-in 


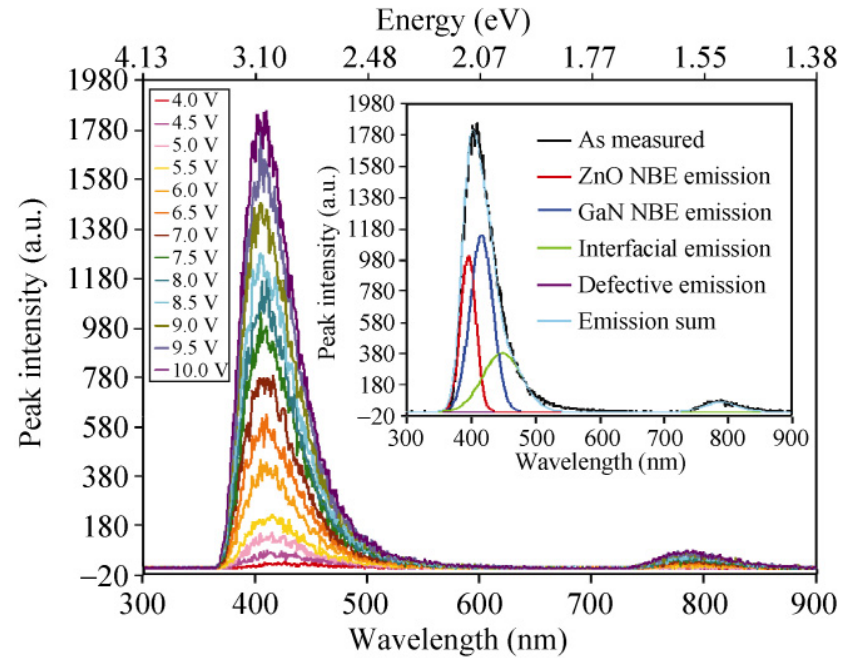

(a)

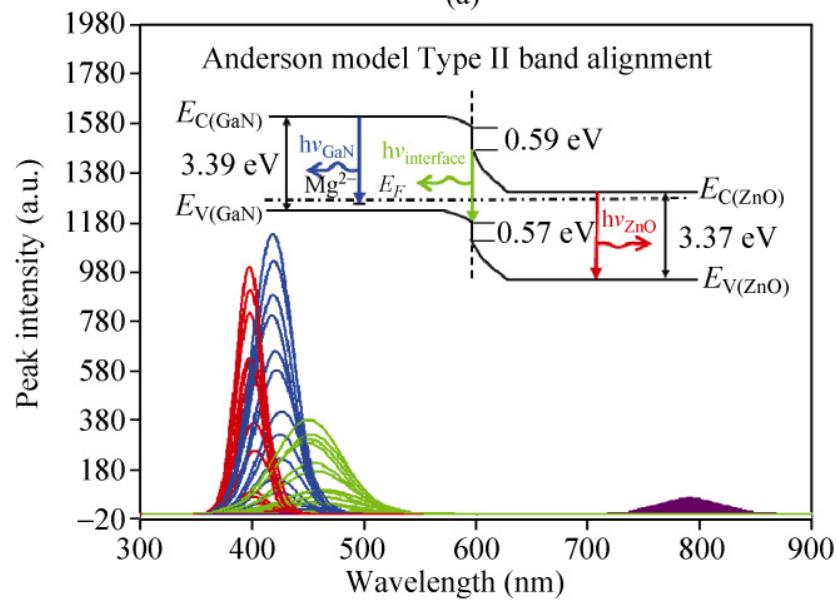

(b)

Figure 45 (a) Electroluminescence spectra as a function of the forward bias voltage. Inset shows how by Gaussian deconvolution analysis the blue/near-UV emission can be decomposed into three distinct bands that correspond to three different optoelectronic processes. (b) All of the four emission bands evolve as a function of the bias voltage. Inset schematics show the band diagram of the $n-\mathrm{ZnO} / p-\mathrm{GaN}$ heterojunction under no or small bias voltage, where the three emission bands making up the blue/near UV band are indicated in different colors [371]. Reproduced with permission

piezoelectric field [384]. As shown in Fig. 46, the blueshift of the emission peak came has two origins: the blue-shift in emission band positions, and the change in relative intensities of the luminescence at different injection currents [380]. The blue-shift of the $\mathrm{ZnO}$ and GaN near-band-edge emission positions (Fig. 46(a) i and ii) might be caused by the increasing kinetic energies of the electrons and holes as the bias voltage was increased. As the bias voltage was gradually increased, the kinetic energy of the free carriers going across the barrier was accordingly increased (assuming the mobility of the carriers did not change too much when the bias voltage was increased), which could contribute to the energy of the emitted photons. The most obvious blue-shift of the peak position came from the interfacial recombination process (Fig. 46(a) iii), which was simply because the increases in kinetic energies from both electrons and holes contributed to the interfacial recombination.

The blue-shift of the electroluminescence spectrum also resulted from the change in the relative intensity of the four bands, as shown in Fig. 46(b). The intensity of the GaN near-band-edge emission increased fastest among the four sub-bands. As the bias voltage was increased, the band bending of the $p-\mathrm{GaN}$ and $n-\mathrm{ZnO}$ was reduced, therefore the kinetic energies of the
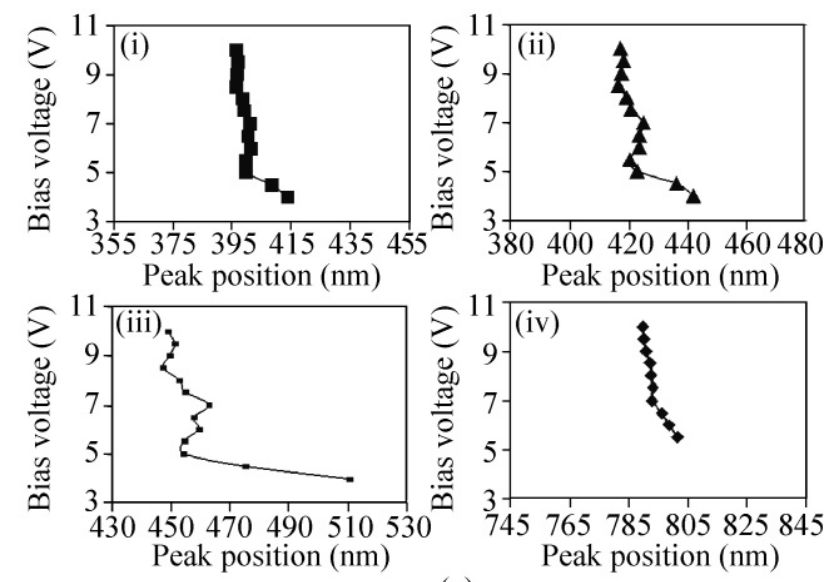

(a)

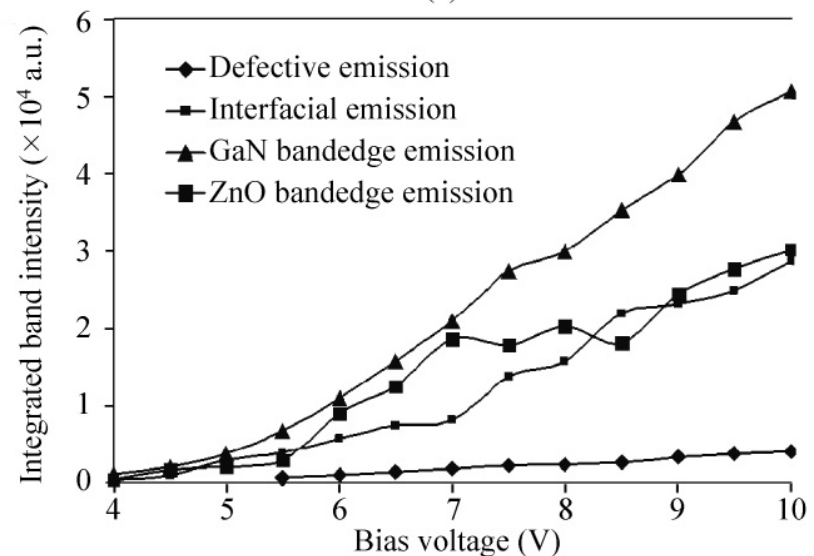

(b)

Figure 46 (a) Blue-shift of peak positions as a function of the bias voltage for (i) $\mathrm{ZnO}$ near-band-edge emission, (ii) GaN nearband-edge emission, (iii) interfacial emission, and (iv) defect emission. (b) The integrated emission band intensities as a function of the bias voltage. When the bias voltage is below $5.5 \mathrm{~V}$, the defect emission can hardly be distinguished from the noise level [371]. Reproduced with permission 
electrons and holes were increased giving them a much higher probability of going across the interface barrier and recombining at the other side. Comparison of the curve for $\mathrm{ZnO}$ near-band-edge emission and that for GaN near-band-edge emission (Fig. 46(b)) shows that, at low bias voltages $(<7.0 \mathrm{~V})$, the $\mathrm{ZnO}$ near-band-edge emission had a competitive increase in intensity relative to GaN. As the bias voltage was increased, the $\mathrm{ZnO}$ near-band-edge emission fell behind that of GaN. In comparison with a high quality GaN thin film fabricated by MOCVD, the relatively high level of native defects in the hydrothermally grown $\mathrm{ZnO}$ nanowires undermines its internal quantum efficiency, especially when the bias voltage is relatively high. These point defects in the $\mathrm{ZnO}$ nanowires act like trapping centers and give rise to nonradiative recombinations.

\subsubsection{Inorganic-organic hybrid LEDs}

$\mathrm{ZnO}$ nanowire arrays can be grown on flexible polymer substrates at low temperature and low cost on a large scale by electrochemical deposition $[385,386]$ or seeded growth [95]. When filled with insulating polymers as a supporting matrix, these $\mathrm{ZnO}$ nanowires can hybridize with $p$-type organic polymers to give flexible and foldable LEDs or materials for photovoltaic applications [249]. This provides an interesting alternative to all-organic counterparts. In fact, they are probably superior to their all-organic counterparts in the sense that the active optical component in such hybrid structures is the inorganic material, which usually has a higher carrier mobility and lifetime than organic materials [386]. Also, stability in air may be better than for all-organic devices [385, 387].

Konenkamp et al. demonstrated a $\mathrm{ZnO}$ nanowire/ polymer hybrid LED [385]. The device structure is illustrated schematically in Fig. 47(a). ZnO nanowire arrays were grown on fluorine doped tin oxide (FTO) glass by electrochemical deposition. Similar arrays can also be achieved on flexible polymer substrates [249, 386, 388]. Annealing at around $300{ }^{\circ} \mathrm{C}$ helps to increase crystallinity and surface roughness of the nanowires, which leads to an increase in the excitonic emission of the electroluminescence. Addition of $\mathrm{AlCl}_{3}$ to the deposition electrolyte was found to improve the conductivity of the nanowires, resulting in an increased emission intensity [249]. During the electrodeposition (a)

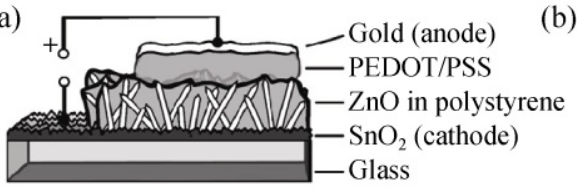

(b)
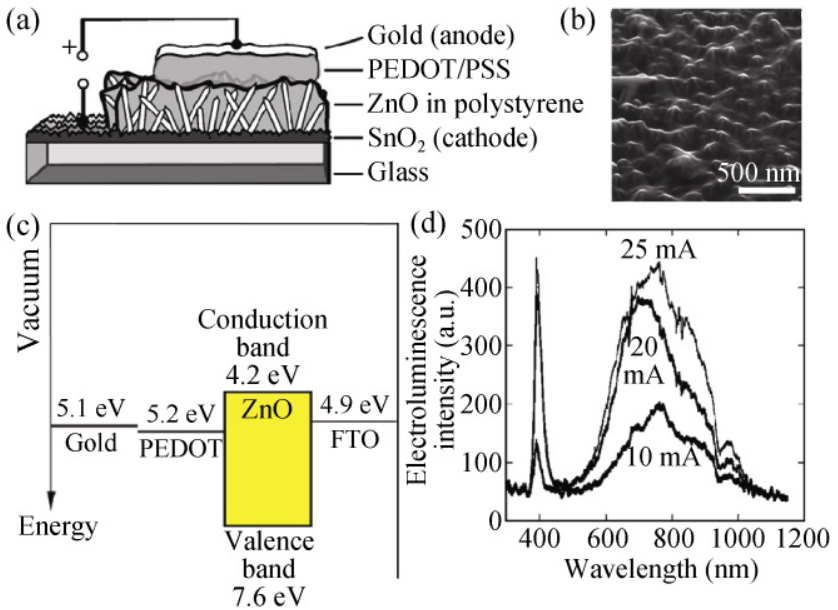

Figure 47 (a) Schematic diagram of a hybrid LED structure [385]. (b) SEM image of the $\mathrm{ZnO}$ nanowires embedded in an insulating polymer matrix [385]. (c) Energy levels of the components in the hybrid LED [386]. (d) Electroluminescence spectra at different injection currents obtained at room temperature in air. The labeled current values are for a device area of $0.3 \mathrm{~cm}^{2}$, and the $\mathrm{ZnO}$ nanowires have been annealed [386]. Reproduced with permission

process, electrons flowed continuously from the FTO substrate to the $\mathrm{ZnO}$ nanowires, so good contact was formed between the nanowires and the underlying substrate. The nanowires were then encapsulated with a thin layer of insulating polymer as shown in Fig. 47(b), which is often the case when making top electrodes of nanowire arrays. Polymers adhere well to the oxide surfaces, and by adjusting the spin speed, the polymer molecular mass, and the polymer concentration, the thickness of the polymer on the nanowire tip can be varied from $10-50 \mathrm{~nm}$, which is sufficiently thin that electric transport through the layer can occur via electronic and structural defects $[385,388]$. Oxygen plasma can also be used to etch away the polymer on the nanowire tip and expose fresh $\mathrm{ZnO}$ nanowire tips [386].

A diode was fabricated by spin/spray coating a layer of strongly $p$-doped poly(3,4-ethylene-dioxythiophene)/ poly (styrenesulfonate) (PEDOT:PSS) on top of the nanowire tips. Typically the $p$-type polymer layer was about $1 \mu \mathrm{m}$ in thickness. Top contact to the $p$-type polymer was made by depositing a semitransparent layer of $\mathrm{Au}$ that needs to be thin enough to allow light transmission yet thick enough to maintain its conductance integrity. The similarity between the 
photoluminescence and the electroluminescence spectra of the annealed $\mathrm{ZnO}$ nanowire indicated that the $\mathrm{ZnO}$ nanowires were the active material where radiative electron and hole recombination took place. The energy level diagram is shown in Fig. 47(c). There was a $2.4 \mathrm{eV}$ barrier for hole injection from the PEDOT:PSS to the $\mathrm{ZnO}$ valence band edge. Multiple layers of $p$-type polymers were used between PEDOT:PSS and $\mathrm{ZnO}$ as band offset stepping layers to ensure effective hole transport and electron blocking [389, 390]. There was a $0.7 \mathrm{eV}$ barrier for electron injection from the FTO to the $\mathrm{ZnO}$ conduction band edge, so a minimum forward bias voltage of $3.1 \mathrm{~V}$ was required to drive the device. Luminescence was observed experimentally at around 5 to $7 \mathrm{~V}$. In the operational voltage regime, the injection current was found to be proportional to the square of the bias voltage, which can be explained by the spacecharge-limited injection [249]. Fitting the experimental data with the Mott-Gurney law suggested that the $\mathrm{ZnO}$ nanowires are trap-free [249]. High voltage $(\sim 40 \mathrm{~V})$ scanning has been shown to be beneficial for electrical conductance by virtue of its effect at the interfaces of the different layers [385]. The emission spectra of the hybrid LED after the $\mathrm{ZnO}$ nanowires were annealed are shown in Fig. 47(d) [386]. The emission covers most of the visible range, giving rise to a white color. In addition, there was a narrow UV band centered at $393 \mathrm{~nm}$ with a FWHM of about $24 \mathrm{~nm}$. Even without further encapsulation, the device was shown to be stable over $1 \mathrm{~h}$ under ambient conditions [385].

Beside PEDOT:PSS, other $p$-type polymers have also been used as the hole injection layer, such as poly(3methylthiophene) [391] and many others [392]. Sun et al. used $N, N^{\prime}$-di(naphth-2-yl)- $N, N^{\prime}$-diphenylbenzidine (NPB), for which the lowest unoccupied molecular orbital is at a higher level than that of PEDOT:PSS, and enhances hole transport as well as electron blocking. Electrons cannot flow from $\mathrm{ZnO}$ to NPB and are accumulated on the $\mathrm{ZnO}$ side near the $\mathrm{ZnO} / \mathrm{NPB}$ interface, and gradually fill up the conduction band of $\mathrm{ZnO}$, which eventually results in a blue-shifted and broadened excitonic emission at $342 \mathrm{~nm}$ [393].

\subsubsection{Schottky diode-based LEDs}

Like $p-n$ junctions, Schottky diodes can also produce electroluminescence under bias, which was first experi- mentally observed for SiC [394]. Since then, Schottky diode-based LEDs have been reported for various materials under either forward or reverse biases, including ZnSe [395, 396], porous Si [397], GaN [398], as well as $\mathrm{ZnO}$ nanowires and nanotubes [399-401].

The working principle of Schottky type LEDs is illustrated in Fig. 48. To take a $n$-type $\mathrm{ZnO}$ nanowire/ $\mathrm{Au}$ electrode as an example, a Schottky diode is formed under equilibrium. When under forward bias above the threshold voltage, electrons will diffuse from the $\mathrm{ZnO}$ into the $\mathrm{Au}$. When the forward bias is further increased, of course more electrons will diffuse across the interface. It is also possible that holes will be injected from the $\mathrm{Au}$ into the $\mathrm{ZnO}$ valence band when the $\mathrm{ZnO}$ valence band is above the Au Fermi level under high forward bias voltage. To put it in a different way, electrons are injected from the $\mathrm{ZnO}$ valence band to the $\mathrm{Au}$, leaving holes in the $\mathrm{ZnO}$ valence band. These holes will then recombine with electrons giving rise to near-band-edge emission. When the Schottky diode is strongly reverse biased, electrons can tunnel through the Schottky barrier and inject into the $\mathrm{ZnO}$ conduction band. These energetic electrons will cause impact ionization which generates more electron and hole pairs by an avalanche effect. Electron and hole radiative recombination enables near-band-edge emission in $\mathrm{ZnO}$.

In the study by Wang et al., the Schottky diode was formed between $\mathrm{ZnO}$ nanowire arrays and a semitransparent $\mathrm{Au}$ top electrode [399]. Electroluminescence was observed at a forward bias voltage of $6 \mathrm{~V}$ at room temperature. The electroluminescence spectrum consisted of two peaks: one centered at $392 \mathrm{~nm}$ and the other at $525 \mathrm{~nm}$, corresponding to excitonic nearband-edge and defect emission, respectively [399]. The electroluminescence near-band-edge emission (392 nm in this case) was red-shifted in comparison with the photoluminescence peak (378 $\mathrm{nm}$ in Fig. 40(a)). This was because structural defects, such as point defects and surface dangling bonds in nanowires, can form band-tail states near the band edge. The amount of excess carriers injected by the electric current is far less than that excited by the laser. As a result, the low density of electrically injected carriers mainly fill the band-tail states, leading to the red-shift of near-bandedge emission [402]. 


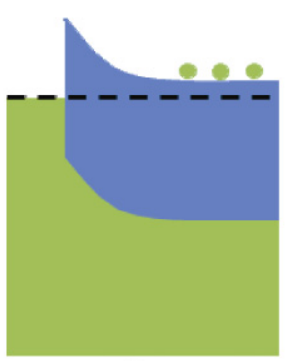

(a)

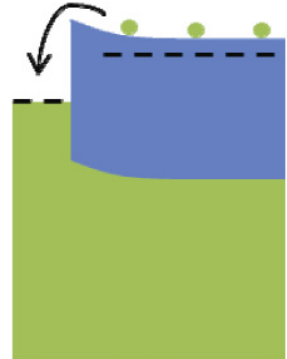

(b)

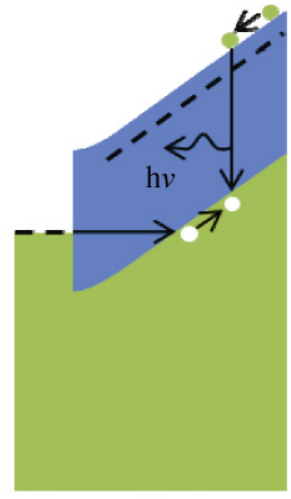

(c)

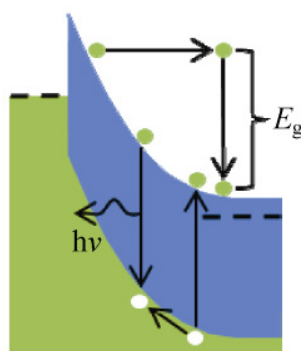

(d)

Figure 48 Schematic band diagrams showing the working principle of Schottky diode-based LEDs. (a) Under equilibrium, (b) under forward bias above the threshold, (c) under strong forward bias, and (d) under strong reverse bias

\subsubsection{Light extraction}

Due to the high refractive index of the active materials, flat thin film-based LEDs suffer from low light extraction efficiency limited by the total internal reflection [403]. Extensive efforts have been made to tackle this problem, such as roughening the emitting surface of the thin film [404, 405], reshaping the geometry of the LED architecture [406, 407], utilizing a resonant cavity [408], coupling with surface plasmons [409], fabricating photonic crystal structures [410, 411], and using corrugated Bragg gratings [412]. Nanowire/ thin film heterostructures have been proposed to be a promising solution, considering the waveguiding properties of the nanowires that should enhance light extraction efficiency of the LED's [9, 413-418].

For the case of a $\mathrm{ZnO}$ nanowire/GaN substrate configuration, under single mode waveguide cavity conditions [419, 420], $86.5 \%$ of the light can be confined within a nanowire of diameter of $223 \mathrm{~nm}$ :

$$
V=2 \pi \frac{a}{\lambda_{\mathrm{o}}}\left(n_{1}^{2}-n_{2}^{2}\right)^{\frac{1}{2}}=2.405
$$

where $V$ is the single mode cut-off value, $a$ is the diameter of the nanowire, $\lambda_{\mathrm{o}}$ is the wavelength of the propagating light $(\sim 400 \mathrm{~nm})$, and $n_{1}$ and $n_{2}$ are the effective refractive index of the $\mathrm{ZnO}$ nanowire and the cladding PMMA thin film, respectively [371]. The facets of the nanowire were assumed to be perfectly flat under the single mode conditions. In reality, the nanowires which were grown by wet chemical methods usually had rounded tips and rough surfaces, large surface to volume ratio, and thus a high density of surface states near the band edge, which allowed a large reduction in the back reflections at the $\mathrm{ZnO}$ nanowire surfaces, known as the omnidirectional reflector effect [421]. The refractive indices of $\mathrm{GaN}, \mathrm{ZnO}, \mathrm{PMMA}$, and air are 2.49, 2.10, 1.59, and 1.00, respectively. Based on the effective medium theory, graded refractive indices can largely reduce the Fresnel reflection between $\mathrm{GaN} / \mathrm{ZnO}, \mathrm{ZnO} / \mathrm{PMMA}$, and PMMA/air interfaces, which should benefit optical transmission. By virtue of such advantages provided by the nanowires, the light can easily be extracted by multiple scattering. $\mathrm{ZnO}$ had a transmittance of over $90 \%$ in the visible range [414], so self-absorption by the nanowires should not be a major concern.

Figure 49 shows the room temperature external quantum efficiency (calculated by from the ratio of the output light power to the input electrical power) versus d.c. injection current for the nanowire/thin film heterostructure LED [371]. The external quantum efficiency of the LED was about $2.5 \%$, which is very high for a single $p-n$ junction-based LED. As the bias voltage/injection current was gradually increased, the external quantum efficiency became steady, indicating that the serial resistance or the nonradiative recombination through the defects, e.g., Auger recombination, did not increase in proportion with the increasing injection current.

The patterned $\mathrm{ZnO}$ nanowire arrays formed a quasi 2D photonic crystal, which potentially have a photonic band gap for light traveling parallel to the surface of 


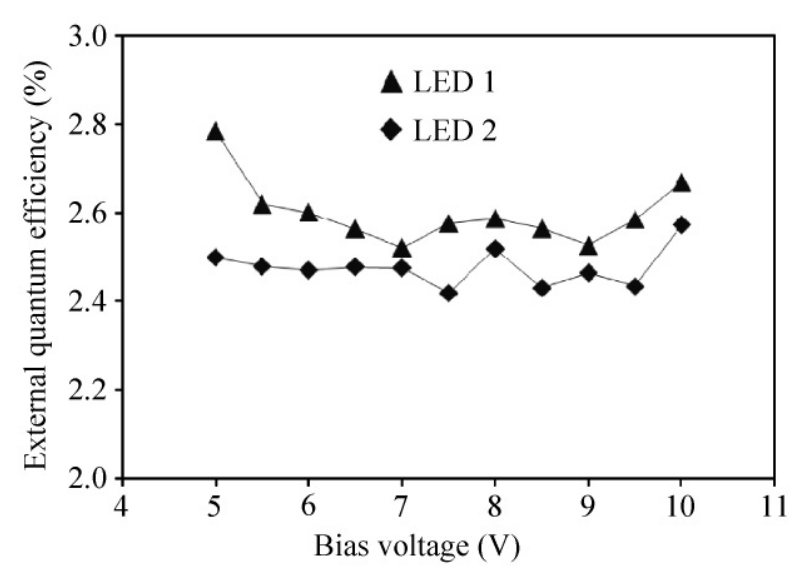

Figure 49 External quantum efficiencies of two $\mathrm{ZnO}$ nanowire/ $\mathrm{GaN}$ thin film heterostructural LEDs as a function of the bias voltage/injection current [371]. Reproduced with permission

the substrate. Therefore by controlling the pitch of the nanowire array, it might be possible to match the wavelength of the electroluminescence to the band gap of the photonic crystal. Kee et al. used finite difference time domain simulations to show the light propagation behavior within the photonic crystal [340]. Figure 50 shows the spatial distribution of a TM mode electric field in a quasi-2D triangular $\mathrm{ZnO}$ nanowire photonic crystal, when the photon frequency is inside and outside the photonic band gap. When the photon frequency was outside the photonic band gap, the photon propagated freely in any in-plane direction. When the photon frequency was inside the photonic band gap, however, the photon did not propagate, all in-plane guiding modes were decayed to zero, and the photons were coupled entirely to the free space modes. This resulted in an enhanced extraction efficiency, and also light emission in a normal direction [340].

\subsection{Electrochromic displays}

Electrochromic devices undergo color change on charge injection or extraction driven by an external voltage [423]. They have low cost and low power consumption, high coloration efficiency, and memory effects under open circuit states. There are generally four categories of electrochromic devices [424]. Sun et al. demonstrated a fast-switching electrochromic device based on a viologen-decorated $\mathrm{ZnO}$ nanowire array electrode [422]. The schematic structure of the device is shown in Fig. 51. The $\mathrm{ZnO}$ nanowires were grown on a

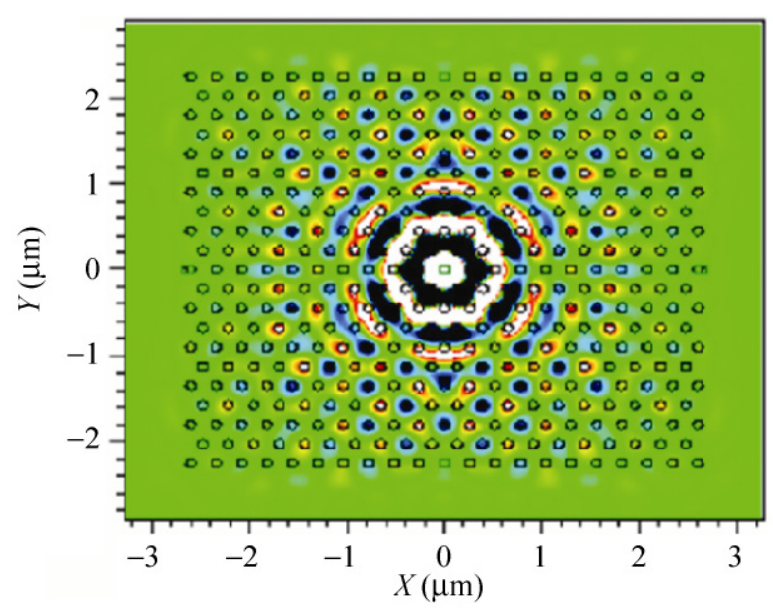

(a)

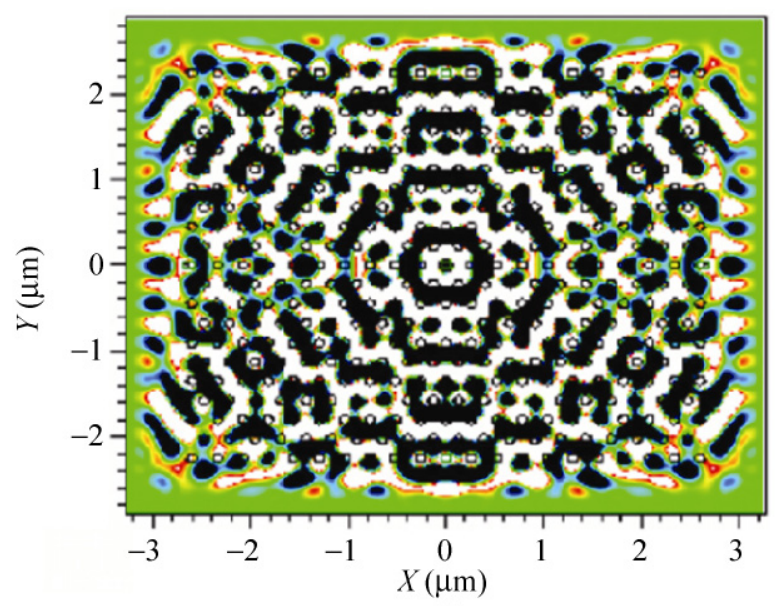

(b)

Figure 50 Spatial distribution of the electric field radiated from a point source at the center of a $2 \mathrm{D}$ triangular $\mathrm{ZnO}$ nanowire photonic crystal, when the frequency of the photon is (a) inside and (b) outside the photonic band gap [340]. Reproduced with permission

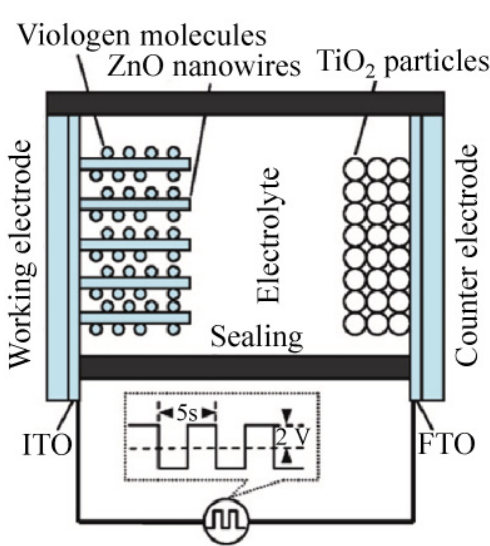

(a)

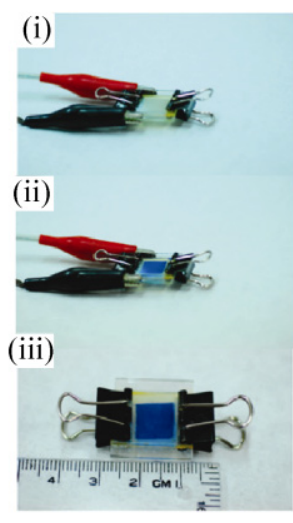

(b)
Figure 51 (a) Schematic structure of the electrochromic device, and (b) photographs of the electrochromic device (i) before and (ii) after applying an external voltage, and (iii) at the open circuit state [422]. Reproduced with permission

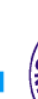

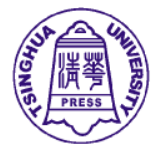

Springer 
transparent conductive substrate by a wet chemical method. Then the organic chromophore-methyl viologen molecules in this case-were loaded onto the nanowires by immersing them in a methyl viologen solution. Unattached viologen molecules were rinsed away, and only those that were physically/chemically adsorbed on the nanowire surface were left to ensure a direct charge transport between the nanowire and the viologen molecule. Therefore the switching speed of the device was very fast, and the nanowire itself provided a direct charge transport path. The electrolyte was made of $\mathrm{LiClO}_{4}$ in $\gamma$-butyrolactone. The nanowires decorated with viologen molecules were used as the working electrode, which was sealed together with a reference electrode since the redox reaction of the viologen molecules is sensitive to oxygen. When under negative bias, the viologen molecules were reduced and become deep blue within about $170 \mathrm{~ms}$, and the color was retained for over $1 \mathrm{~h}$ after the bias was removed. When under positive bias, the bluish color was bleached within about $142 \mathrm{~ms}$. The switching time became shorter for larger bias voltage and smaller device size. The device performance was also dependent on the nanowire density [425].

\subsection{Field effect transistors}

Field effect transistors (FETs) based on a single $\mathrm{ZnO}$ nanowire [426] or $\mathrm{ZnO}$ nanowire thin films [427] have been fabricated for use in optically transparent mechanically flexible electronics [428, 429]. The fact that $\mathrm{ZnO}$ nanowires can grow in situ between the source and drain electrodes is one of the merits of low temperature wet chemical methods [283, 430, 431]. Ko et al. demonstrated an all-solution fabrication process for a $\mathrm{ZnO}$ nanowire transistor on polymer substrates, as shown in Fig. 52(a) [430]. The source and drain electrodes were fabricated by nanoimprint lithography of Au nanoparticles followed by low temperature soft annealing. $\mathrm{ZnO}$ nanoparticles were applied to the patterned Au electrodes, and later seeded the growth of $\mathrm{ZnO}$ nanowires. Depending on the channel width between the source and drain electrodes, the $\mathrm{ZnO}$ nanowires could grow and connect the two electrodes through forming complex nanowire networks, as shown in Fig. 52(b). Even though there is a work function mismatch between $\mathrm{Au}(5.1 \mathrm{eV})$ and $\mathrm{ZnO}(4.5 \mathrm{eV})$, the
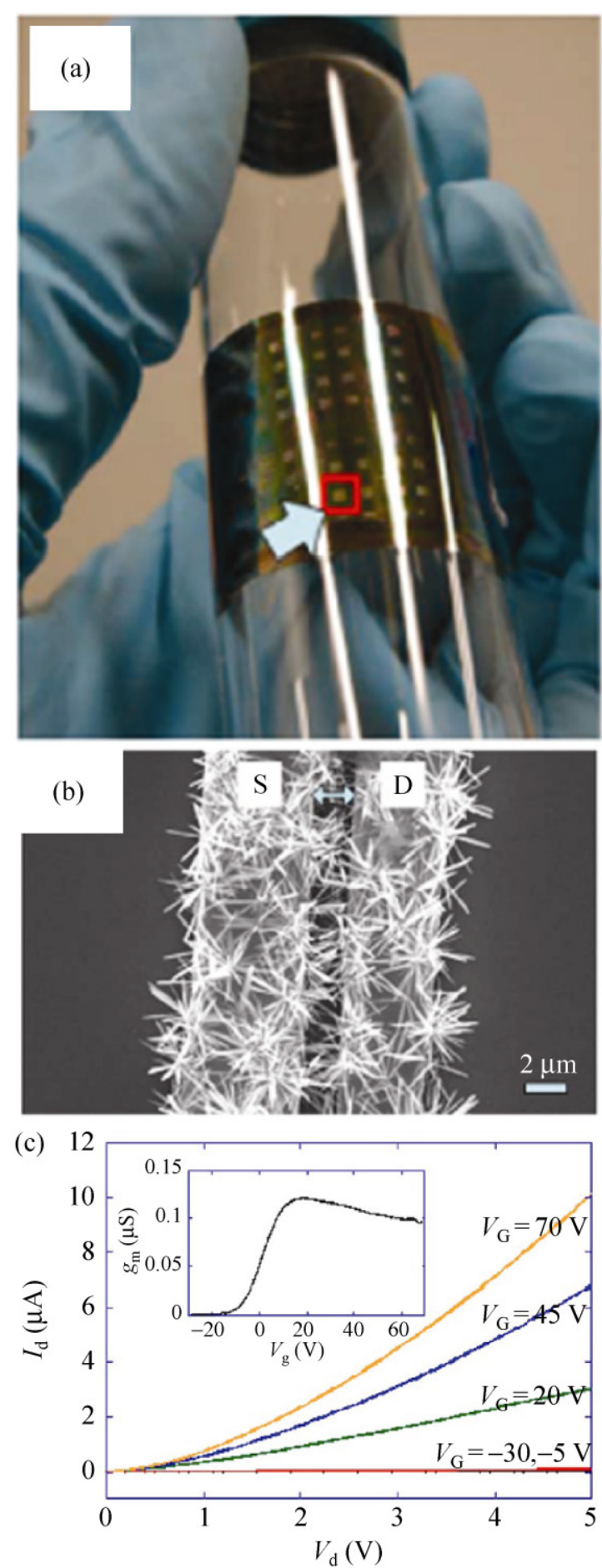

Figure 52 (a) A digital photograph of $\mathrm{ZnO}$ nanowire transistor arrays on a flexible substrate. The red square includes an array of thirty-six transistors. (b) SEM image of the $\mathrm{ZnO}$ nanowires grown in situ between the source and drain electrodes. (c) Output characteristics of eighteen $\mathrm{ZnO}$ nanowire transistors with $10 \mu \mathrm{m}$ channel length. The inset shows a plot of transconductance as a function of gate voltage [430]. Reproduced with permission 
contact between them should be ohmic because during the wet chemical growth process, the interface is not perfect. Therefore the transport properties are not determined by the intrinsic work function difference, but rather by the interfacial defect states [432]. The output characteristics and averaged transconductance $g_{\mathrm{m}}$ are shown in Fig. 52(c), which shows an $n$-type channel with an on/off current ratio of $10^{4}-10^{5}$. The maximum value of $g_{\mathrm{m}}$ was about $100 \mathrm{nS}$. The mobility $\mu_{\mathrm{e}}$ of the electrons in the nanowire was calculated to be $0.2 \mathrm{~cm}^{2} / \mathrm{V} \cdot \mathrm{s}$, according to $\mu_{\mathrm{e}}=g_{\mathrm{m}}{ }^{2} L / 2 I_{\mathrm{d}} C_{\mathrm{ox}} W$, where $C_{\text {ox }}$ is the gate capacitance, and $L$ and $W$ are the channel length and width, respectively. Konenkamp et al. reported that the carrier mobility in the undoped $\mathrm{ZnO}$ thin film was as high as $23 \mathrm{~cm}^{2} / \mathrm{V} \cdot \mathrm{s}$ [118], but that was probably due to the nanowire surface state scattering-induced decrease in electron mobility. The $\mathrm{ZnO}$ nanowire transistor did not show a saturation regime as the drain voltage was increased, probably because of the dominant phonon scattering and the impurity-derived grain boundaries in the bridged $\mathrm{ZnO}$ nanowires [431]. Of course, the FET characteristics were dependent on the alignment of the $\mathrm{ZnO}$ nanowires [427].

\subsection{Sensors}

$\mathrm{ZnO}$ nanowires have been used as active components in gas sensors [167, 168, 433], visible light sensors [434], visible-blind UV sensors [95, 280, 435], biosensors [94, 436], and strain sensors [437]. These depend on the resistance or piezoresistance change of the nanowires [438], or the barrier height change at the contact [15-17, 437, 439], or are based on the heterostructured $p-n$ diodes [434].

$\mathrm{ZnO}$ is amphoteric, with a isoelectric point of about 9.5 [54]. The $\mathrm{ZnO}$ surface will be functionalized with protons $\mathrm{H}_{3} \mathrm{O}^{+}$or hydroxyl $\mathrm{OH}^{-}$groups with different $\zeta$ potentials, when the $\mathrm{pH}$ value is lower or higher than the isoelectric point, respectively. By utilizing $\mathrm{ZnO}$ nanowires as an electrochemical probe, Al-Hilli et al. reported an intracellular $\mathrm{pH}$ sensor [440]. The benefits of using $\mathrm{ZnO}$ nanowire arrays are that they have high surface to volume ratio and offer high sensitivity and real-time detection. In Al-Hilli's experiment, $\mathrm{ZnO}$ nanowire arrays were grown on a sharp borosilicate glass tip as the working electrode, and $\mathrm{Ag} / \mathrm{AgCl}$ was used as the reference electrode. The basic working principle is that a $\mathrm{pH}$ change in the surroundings leads to a surface potential change in the nanowire, which is detected as a voltage change between the working and reference electrodes. The two electrodes were first calibrated with standard $\mathrm{pH}$ buffer solutions at room temperature. As shown in Fig. 53(a), the electrochemical potential between the two electrodes was almost linearly proportional to the buffer electrolyte $\mathrm{pH}$ within the $\mathrm{pH}$ range $4-11$, with a sensitivity of about $52 \mathrm{mV} / \mathrm{pH}$. The authors noted that the $\mathrm{ZnO}$ nanowires were thermodynamically stable over the tested $\mathrm{pH}$ range at room temperature [440]. For the measurement of intracellular $\mathrm{pH}$ value, both electrodes were then pushed to penetrate the membrane of a human fat cell. As shown in Fig. 53(b), the two electrodes were in direct contact with the cell cytoplasm, and the intracellular $\mathrm{pH}$ value was read to be 6.81 . $\mathrm{ZnO}$ is

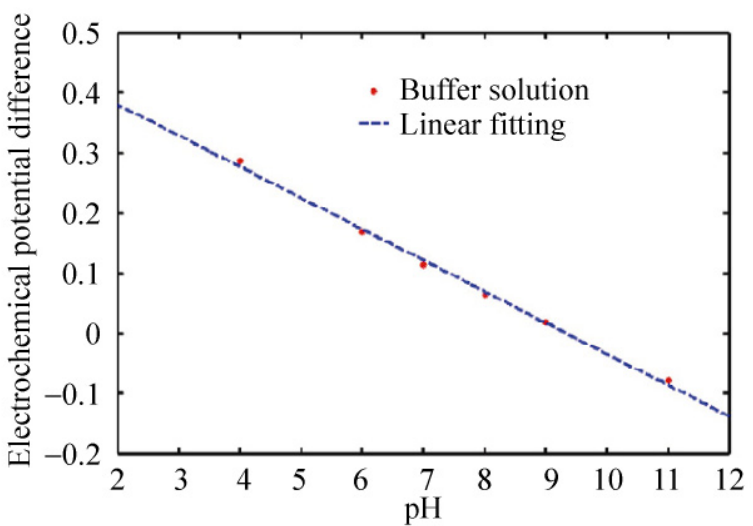

(a)

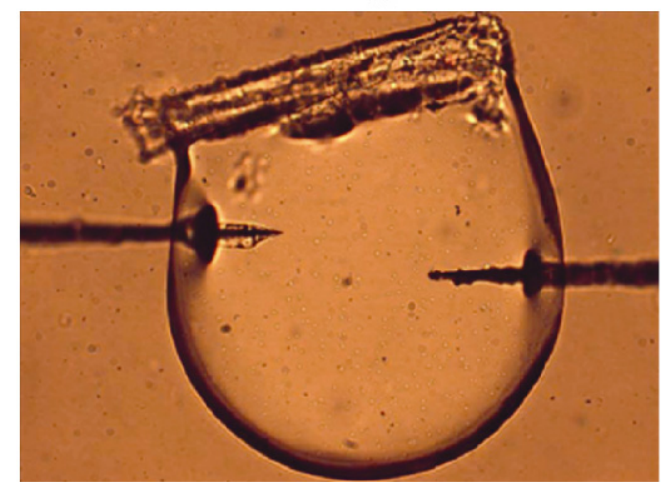

(b)

Figure 53 (a) Calibration curve of the two electrodes in standard buffer solution. (b) Optical image showing the intracellular $\mathrm{pH}$ measurements in a single human fat cell [440]. Reproduced with permission 
nontoxic and biocompatible [441, 442]. The intracellular measurement did not visibly affect the cellular viability so this technique could be a good way of in situ $\mathrm{pH}$ measurement in single living cell.

\subsection{Energy harvesting devices}

$\mathrm{ZnO}$ nanowire arrays are playing a very active role in energy harvesting technologies nowadays. Preliminary proof-of-concept devices include solar cells, piezoelectric nanogenerators, and water splitting devices, as shown in the following sections.

\subsubsection{Solar cells}

Silicon-based conventional solar cells have low defect densities and high carrier mobilities, and currently dominate the solar energy industry due to the relatively high energy conversion efficiency and well-developed fabrication technologies [443]. Extensive studies of other inorganic and organic materials have been carried out with the aim of further reducing the cost per unit energy output. ZnO nanowire arrays are good candidates in particular for solar cell applications for three straightforward reasons: they have low reflectivity that enhances the light absorption, relatively high surface to volume ratio that enables interfacial charge separation, and fast electron transport along the crystalline nanowires that improves the charge collection efficiency. $\mathrm{ZnO}$ nanowire arrays have been implemented for both conventional $p-n$ junction solar cells and excitonic solar cells (including organic, hybrid organic-inorganic, dye-sensitized, and nanoparticlesensitized solar cells), as discussed individually in the following sections.

\subsubsection{Enhancement of light absorption}

Antireflection coating layers play an important role in enhancing the device efficiency by increasing the light coupled with the active region of the solar cell $[50,118,138,444] . \mathrm{ZnO}$ is attractive for this purpose because of its good transparency, appropriate refractive index $(n \approx 2)$, and controllable textured growth [444].

Lee et al. reported that highly textured $\mathrm{ZnO}$ nanorod arrays synthesized by a low temperature solution growth method could be used as an efficient antireflective coating [444]. The fundamental principle is that the intricate distribution and the textured surface of the nanorods ensures a gradual reduction of refractive index from the surface to air, and leads to impedance matching between the substrate and air, which results in photon trapping and reflection suppression over a broad wavelength range. In Lee's study, by changing the growth conditions in the presence of 1,3-diaminopropane, the shape of $\mathrm{ZnO}$ nanorod tips could be modified and slight changes in the tip morphology gave rise to significant changes in antireflection properties. The experimental result was in agreement with simulations by rigorous coupled wave analysis. The reflectance spectra in Fig. 54 show the significant effect on the reflectance of five different samples: sol-gel thin film, an optimized SiN antireflection coating on a Si solar cell, a flat top nanorod array, tapered nanorod array, and a highly tapered nanorod array [444]. To quantify these reflectance curves, weighted reflectance was used. As can be seen from the spectra, the highly tapered nanorod resulted in the strongest broadband suppression of reflectance, with a weighted reflectance value of $6.6 \%$ (black curve). Compared with the flat top nanorod array (red curve), the less tapered nanorod array (orange curve) eliminated the optical interference fringes due to a variation in nanorod length and the ragged $\mathrm{ZnO}$-air

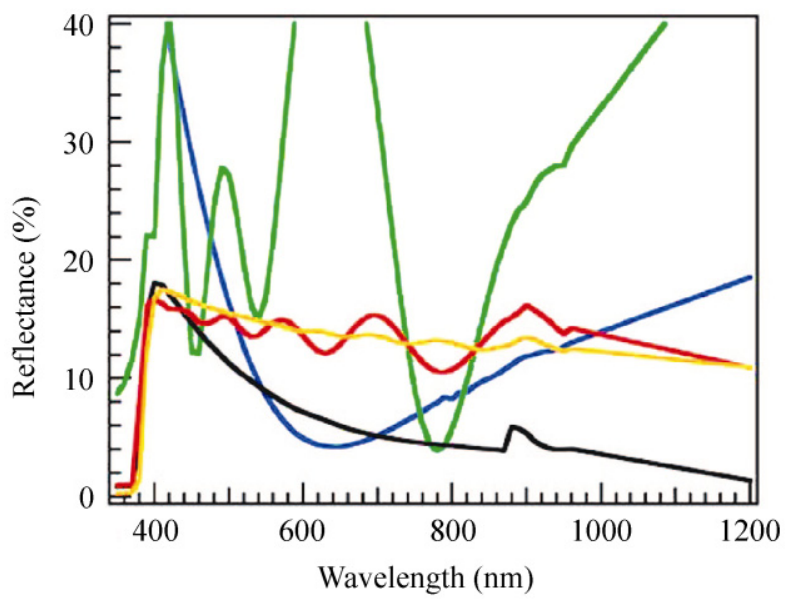

Figure 54 Reflectance spectra of five different samples: sol-gel thin film (green), optimized SiN antireflection coating on Si solar cell (blue), flat top nanorod array (red), tapered nanorod array (orange), and highly tapered nanorod array (black). The little bumps at around $900 \mathrm{~nm}$ on the curves arise from the detector change of the equipment during the date collection [444]. Reproduced with permission 
interface. The optimized SiN antireflection coating on the Si solar cell (blue curve) exhibited a highly wavelength-dependent reflectance response with a minimum at around $640 \mathrm{~nm}$ due to destructive interferences. The sol-gel thin film had a stepwise change of refractive index from the substrate to air, and has the highest weighted reflectance (green curve) [444].

\subsubsection{Dye sensitized solar cells}

High efficiency dye sensitized solar cell (DSSC) have been reported based on $\mathrm{TiO}_{2}$ nanoparticle thin film [445]. In conventional $p-n$ junction solar cells, the semiconductor has the dual functions of light absorption and free carrier transport, whereas in DSSCs the two functions are decoupled. The working principle of a DSSC is shown in Fig. 55. The dye molecules, typically ruthenium complexes [445], or indolene dyes [217], absorb incident photons, go from ground state to excited state, and inject electrons into the conduction band of the semiconductor. The oxidized dye molecules are then quickly neutralized by the redox couples in the electrolyte (typically $\mathrm{I}^{-} / \mathrm{I}_{3}^{-}$), driven by the energy difference between the oxidized dye and the redox couple, which prevents the recapture of electrons from the excited state to the ground state of the dye molecules [446]. The electrons are collected by the semiconductor, flow through the external circuit doing useful work, and return to the counter electrode where the redox couples are reduced and replenished.

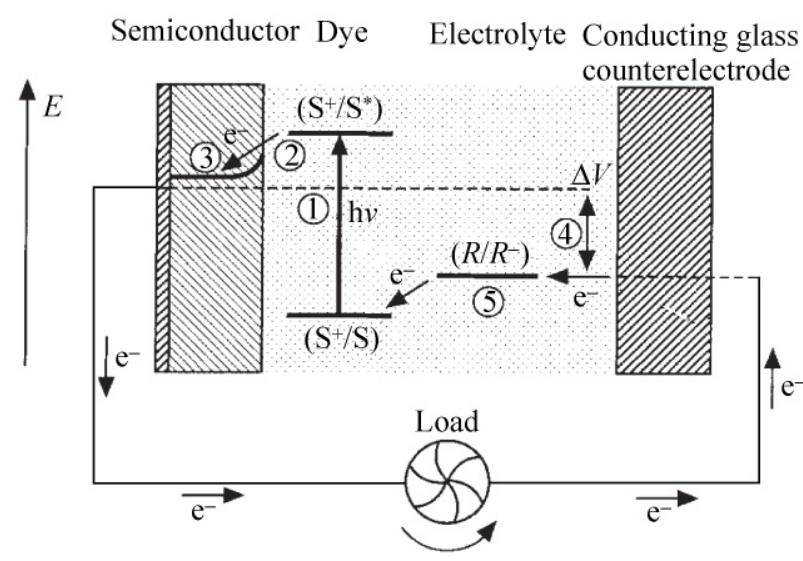

Figure 55 Schematic working principle of a DSSC [445]. Reproduced with permission
Electrons percolate in the sintered $\mathrm{TiO}_{2}$ nanoparticle networks via a series of trap-limited interparticle hopping steps, which greatly decreases the lifetime and diffusion length of the free electrons. Drift transport is prevented to a certain degree in a DSSC because the macroscopic electric field is screened by ions in the electrolyte. In such a case, the electrons must cross an estimated $10^{3}-10^{6} \mathrm{TiO}_{2}$ nanoparticles to reach the substrate electrode [447]. A typical ambipolar diffusion length for the electrons is about $7-30 \mu \mathrm{m}$, so it is disadvantageous to increase the thickness of the active region in the hope of increasing the light absorption.

Electron transport in oriented crystalline nanowires is expected to be several orders of magnitude faster $[448,449]$. The transportation time is on the order of tens of microseconds as revealed by intensitymodulated photocurrent studies [450], by virtue of the high nanowire crystallinity and in particular an internal electric field that assists the exciton separation and charge collection [18, 447]. Based on this idea, Law et al. demonstrated a DSSC based on dense vertically aligned $\mathrm{ZnO}$ nanowires, and the efficiency was boosted up to $1.5 \%$ at a full sun intensity, as shown in Fig. 56(a) [18]. It was shown that nanowires of aspect ratio less than 70 have similar internal quantum efficiencies, indicating that electron transport in the nanowires is not a limiting factor [451]. In spite of their inferior optical properties, hydrothermally grown nanowires exhibited even higher power conversion efficiency than vapor phase grown nanowires due to higher dye adsorption [452]. Other than that, the benefits of nanowires over nanoparticle thin film also include the efficient kinetic electron injection into the nanowires due to the surface fields [222]. Femtosecond transient absorption spectroscopy studies have shown that electron injection from photoexcited ruthenium dyes into nanowires with mainly nonpolar side facets has a much smaller time constant than that for nanoparticles with an ensemble of random surfaces [18]. Also, the nanowires were directly grown on top of the substrate, which ensured an intimate contact for electron transport between the nanowires and the substrate. In addition, a nanowire morphology has a higher resistance to crack formation and propagation than nanoparticle thin films, due to the efficient release of bending stress, which is promising for applications in stable flexible solar cells [453]. 


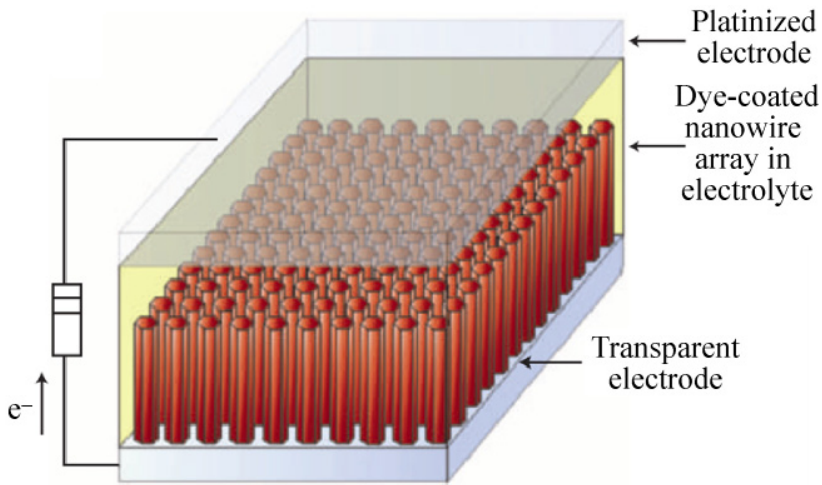

(a)

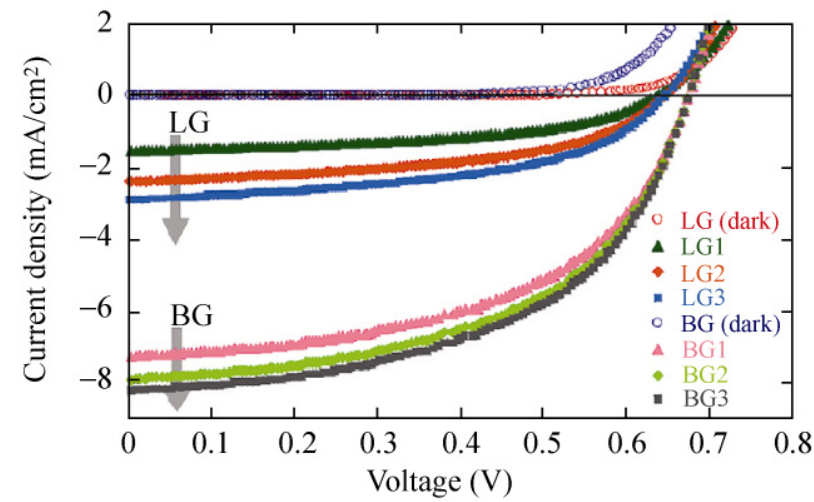

(b)

Figure 56 (a) Schematic diagram of a $\mathrm{ZnO}$ nanowire array DSSC [18]. (b) $J-V$ curves of a DSSC based on hierarchical $\mathrm{ZnO}$ nanowire "nano-forest" with increasing lengthwise growth (LG) and branched growth (BG) [199]. Reproduced with permission

One downside with nanowire array DSSCs is that their internal surface area was found to be only one fifth of that of the nanoparticle thin film [18], which largely limits the cell efficiency [454], so it is helpful to make thinner, longer, and denser nanowires in order to increase the dye loading and the light absorption, and eventually to boost the efficiency. In this regard, studies on nanotubes [455, 456], nanoparticle/nanowire composites [453, 457-460], hierarchical [199, 461] structures [209, 462-464], and different growth parameters [465] have been shown to give several-fold increases in efficiency (Fig. 56(b)).

Conventional solar cells are 2D. The limited surface area significantly constrains the light absorption efficiency. Adopting a different approach, Weintraub et al. demonstrated a three dimensional (3D) DSSC [20]. As shown in Fig. 57(a), the $\mathrm{ZnO}$ nanowires were grown surrounding an optical fiber surface. The light was shone onto the fiber from one end along the axial direction, and underwent multiple interactions with the active materials due to internal reflection within the fiber (Fig. 57(b)), which greatly increase the effective propagation distance and absorption efficiency of the photons. At the same time, the distance electrons had to travel to reach the collecting electrode remained the same. The energy conversion efficiency of the 3D DSSC was enhanced by a factor of up to six compared with the same device when illuminated normal to the optical fiber axial direction. Also, the 3D DSSC could be concealed and located away from the surface, therefore allowing applications at remote locations such as underground or in deep water. Wei et al. extended this strategy one step further by integrating multiple stacks of planar optical waveguides with nanowires [21].

DSSCs offer several manufacturing advantages, including their simple production process, low cost, non-toxicity, and a variety of material resources to choose from [466]. Compared with other solar cells, DSSCs have relatively high efficiency under weak light illumination, so they can be used for internal applications, such as in laptops and mobile phones [466]. The low efficiency of ZnO-based DSSCs probably arises for four reasons: the accumulation of stable $\mathrm{Zn}^{2+} /$ dye complexes instead of chemisorption of the

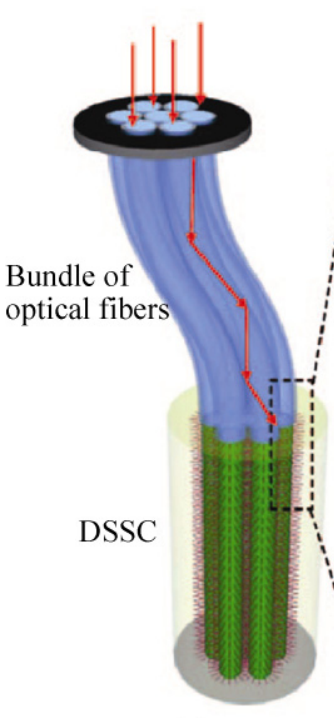

(a)

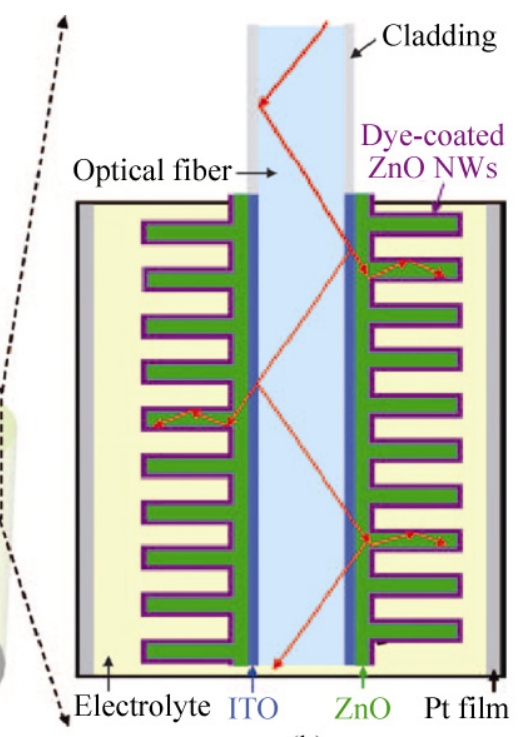

(b)
Figure 57 (a) Schematic design and working principle of a 3D DSSC, and (b) illustration of the multiple interactions with the active materials along the optical fiber [20]. Reproduced with permission 
dye molecule on $\mathrm{ZnO}$ [463]; the low electron injection efficiency induced by the energy level mismatch between the lowest unoccupied molecular orbital (LUMO) of the dye and the photoanode conduction band minimum; the dye regeneration efficiency induced by energy level mismatch between the highest occupied molecular orbital (HOMO) of the dye and the redox mediator potential in the electrolyte [458, 463]; the high carrier recombination rate due to the defect surface trap states of $\mathrm{ZnO}$ nanowires [467], which can be decreased by coating with $\mathrm{TiO}_{2}, \mathrm{Al}_{2} \mathrm{O}_{3}$, or $\mathrm{ZrO}_{2}$ passivating shells $[222,468]$.

\subsubsection{Quantum dot-sensitized solar cells}

Instead of photosensitive dyes, narrow band gap semiconductor quantum dots, such as CdS [469], CdSe [454], and CdTe [470] have also been used to sensitize $\mathrm{ZnO}$ nanowires for use in solar cells. $\mathrm{ZnO}$ has a large direct band gap of $3.37 \mathrm{eV}$ and is transparent in the visible region. Narrow band gap semiconductor materials can absorb visible light in a complementary fashion, and enable the solar cell to cover a wider spectral range. In comparison with organic dyes, quantum dots have several advantages. First, the band gap and thus the absorption spectrum of quantum dots can be tailored by varying the semiconductor material, dot size, or annealing temperature [471], and can be tuned to match the solar spectrum and maximize the light absorption, as shown in Fig. 58(a) [472]. Second, quantum dots have been demonstrated to be able to produce multiple electron-hole pairs per incident photon, which improves the internal quantum efficiency of the device [473, 474]. Third, semiconductors, such as CdS [469] and CdSe [454], typically form a type II heterojunction with $\mathrm{ZnO}$, and the presence of numerous interfaces between the quantum dots and the $\mathrm{ZnO}$ nanowires is greatly beneficial to both light absorption and charge separation. As shown in Fig. 58(a), the surface photovoltage increased with increasing loading of $\mathrm{CdS}$ quantum dots on $\mathrm{ZnO}$ nanowires [472]. Following this strategy, quantum dots sporadically dispersed on the nanowire were replaced with core-shell structures of different materials, such as CdS [475], CdSe [211, 212], and CdTe [213, 476], to further enhance the device efficiency.

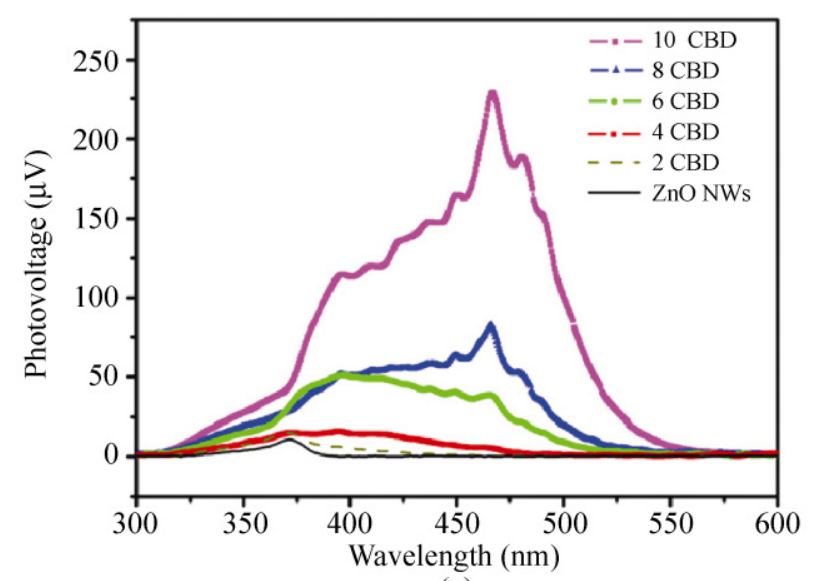

(a)

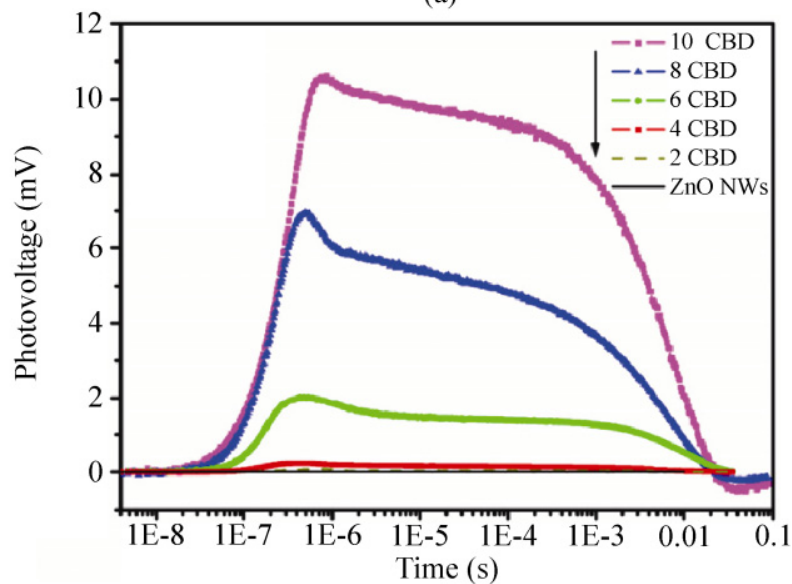

(b)

Figure 58 (a) Surface photovoltage spectra and (b) transient photovoltage spectra of $\mathrm{ZnO}$ nanowire arrays before and after different numbers of chemical bath deposition (CBD) cycles with CdS quantum dots [472]. Reproduced with permission

Electron-hole pairs are generated when incident photons are absorbed by the quantum dots. The electron-hole pairs are confined inside the quantum dots and then separated at the interface, which prevents their recombination. For a typical type II band alignment, electrons are injected from the quantum dot excited state to the $\mathrm{ZnO}$ conduction band, gaining the energy benefits of the difference in electron affinity. Holes are left in the quantum dot which are neutralized by hole conductors or redox couples in the electrolyte, provided that the quantum dot valence band edge is below the hole conductor HOMO or the reductant level of the redox couple. A similar analysis applies when the electron-hole pairs are generated inside the $\mathrm{ZnO}$ nanowires. The charge separation at the quantum dot/nanowire interface is facilitated by the overlap between the quantum dot electronic states 
and the $\mathrm{ZnO}$ electronic bands [454], and normally occurs on a subnanosecond time scale [454], which is shorter than the exciton lifetime. As shown in Fig. 58(b) [472], transient photovoltage studies indicated that the carrier lifetime increased dramatically from microseconds for bare $\mathrm{ZnO}$ nanowires to milliseconds for $\mathrm{ZnO}$ nanowires coated with a CdS sensitizer. Furthermore, as the amount of CdS sensitizer increased, the carrier lifetime increased accordingly [472]. The quantum dots are usually capped with ligands that can form chemical bonds with the $\mathrm{ZnO}$ nanowire surfaces. Therefore the ligand molecule length influences the charge transfer rate at the interface, with short chain lengths having faster transfer rates [477].

\subsubsection{Inorganic $p-n$ junction solar cells}

The built-in electric field at the interface of $p-n$ junctions divorces the photon-excited electron-hole pairs, and provides the basis for conventional inorganic $p-n$ junction solar cells. This is also one of the fundamental differences between conventional $p-n$ junction solar cells and excitonic solar cells: free electrons and holes in conventional solar cells, and excitons (an electronhole pair tightly bound via coulombic forces) in excitonic solar cells. Another fundamental difference is that the open circuit voltage in conventional cells is limited to less than the magnitude of the band bending, while in excitonic solar cells the open circuit voltage is commonly greater than the band bending.

While $\mathrm{ZnO} p-n$ homojunction solar cells have rarely been reported due to the lack of stable $p-\mathrm{ZnO}$ materials, heterojunctions have been employed as an alternative. Cui et al. demonstrated solar cells based on $n-\mathrm{ZnO} / p-\mathrm{Cu}_{2} \mathrm{O}$ nanopillar arrays fabricated by a low cost electrodeposition method [478]. The device structure is shown in Fig. 59(a). The top $p-\mathrm{Cu}_{2} \mathrm{O}$ layer is the absorber due to its narrow band gap of $2.1 \mathrm{eV}$. This configuration maximizes the interface area while maintaining the direct fast transport of electrons along the nanowires, which is reflected by the relatively high short circuit current density in comparison with their thin film counterparts, as shown in Fig. 59(b). The small open circuit voltage was attributed to the dominance of differently exposed crystal facets of the nanowires [478], as also evidenced by surface photovoltage studies [479]. The energy conversion efficiency

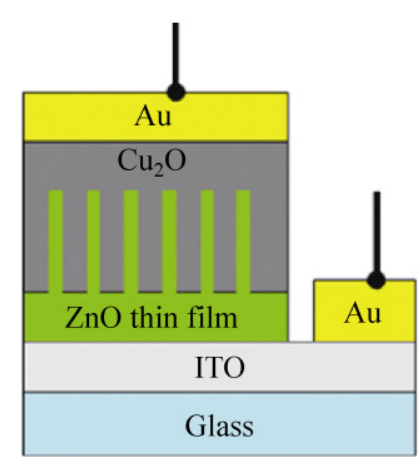

(a)

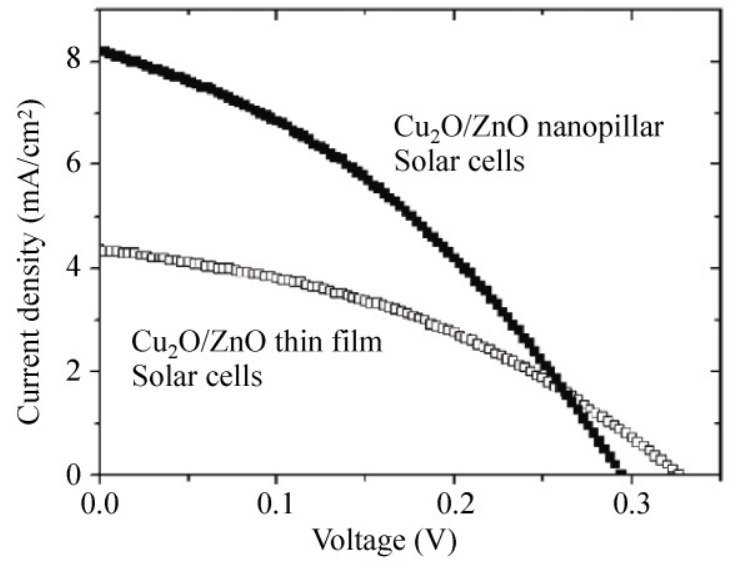

(b)

Figure 59 (a) Schematic structure of a $n-\mathrm{ZnO} / p-\mathrm{Cu}_{2} \mathrm{O}$ solar cell. (b) Typical $I-V$ characteristics of $n-\mathrm{ZnO} / p-\mathrm{Cu}_{2} \mathrm{O}$ nanopillar and thin film solar cells [478]. Reproduced with permission

was found to be $0.88 \%$, which might potentially be increased by many parameters, such as replacing the top Au contact layer that significantly attenuated the incident light intensity.

Combining the working principle of $p-n$ junction solar cells and the advantage of excitonic solar cell, Levy-Clement and coworkers demonstrated the concept of extra thin absorption (ETA) solar cells based on $\mathrm{ZnO} / \mathrm{CdSe} / \mathrm{CuSCN}$ composites [19, 480]. The structure is illustrated in Fig. 60(a). In addition to the conventional $p$-CuSCN $/ n-\mathrm{ZnO}$ junction, there is a conformal layer of CdSe sandwiched in between. The band gap of CdSe $(1.7 \mathrm{eV})$ is much smaller than that of either $\mathrm{ZnO}(3.3 \mathrm{eV})$ or $\mathrm{CuSCN}(3.4 \mathrm{eV})$, so CdSe acts as the absorber. The band diagram of the device is shown in Fig. 60(b). At equilibrium, an electric field was built up across the entire CdSe layer. When the device was illuminated, electrons and holes were generated, separated, and rapidly migrate across the absorber layer. Furthermore, the highly structured 


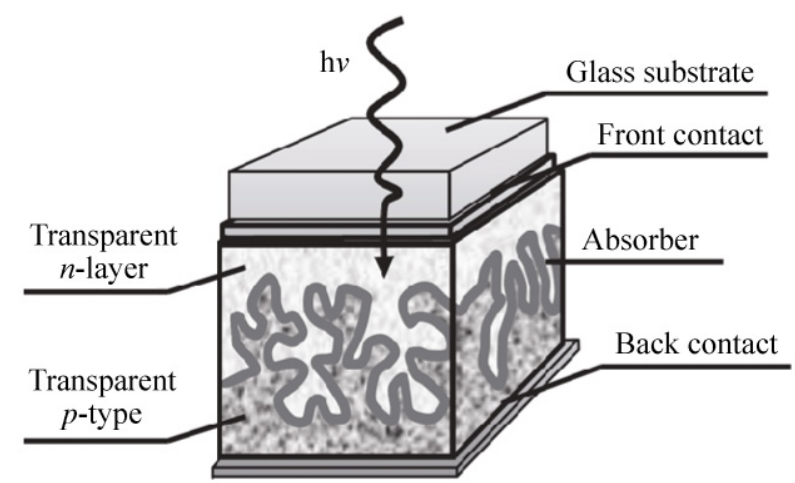

(a)

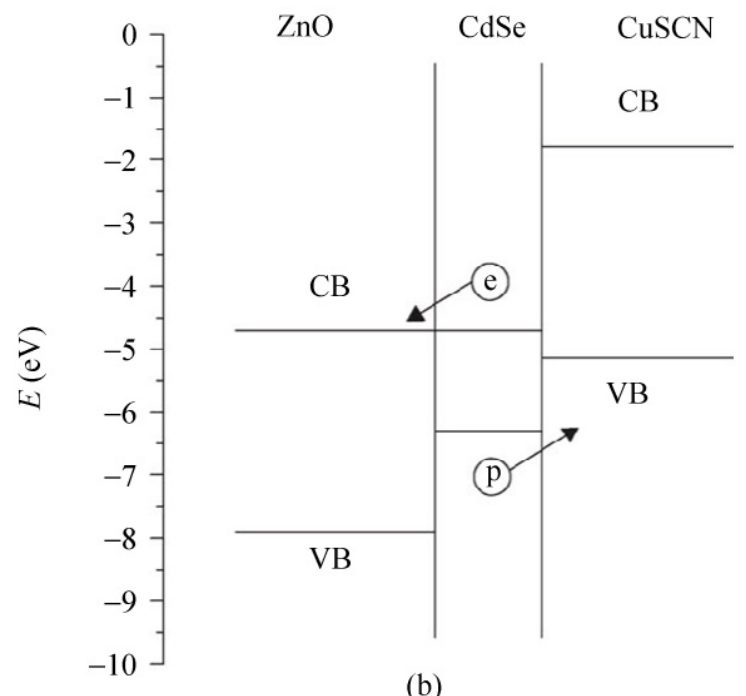

(b)

Figure 60 (a) Schematic structure of the ETA solar cell [19]. (b) Band diagram of the $\mathrm{ZnO} / \mathrm{CdSe} / \mathrm{CuSCN}$ heterostructure [480]. Reproduced with permission

interface substantially reduces the transport distance for the free carriers, and also significantly increases the optical path for the incident photons. In the range $400-800 \mathrm{~nm}$ of the AM1.5 solar spectrum, the averaged absorption and reflectance of this structure were about $89 \%$ and $8 \%$, respectively. Other than that, the all-inorganic composition of the device ensured relatively larger carrier mobility and wider operating temperature than for organic solar cells.

\subsubsection{Hybrid solar cells}

Organic solar cells have reasonably high efficiency, and are great for manufacturing large-scale low-cost flexible solar cells. Due to the very limited carrier mobility of organic semiconductors, the thickness of organic solar cells is typically less than $200 \mathrm{~nm}$, which significantly constrains the incident photon absorption. Organic- inorganic hybrid solar cells have attracted great attention in this regard, since they combine the manufacturing advantages of polymers and the high carrier mobility of inorganic semiconductors. A distinct difference between hybrid solar cells and DSSCs is that there is no liquid electrolyte in the former but there is in the latter [443].

Olson et al. introduced vertically aligned $\mathrm{ZnO}$ nanowire arrays into organic solar cells [481]. The basic structure is shown in Fig. 61(a) [482]. In this hybrid structure, $\mathrm{ZnO}$ nanowire arrays were grown by a low temperature wet chemical method that was compatible with general flexible substrates [483]. The active components were a P3HT:PCBM polymer blend ([6,6]-Phenyl C71 butyric acid methyl (PCBM) ester). $\mathrm{ZnO}$ nanowires performed as "electric fingers" that penetrated into the polymer blend and provided an electron conductance highway with small series resistance [484]. Thus, longer $\mathrm{ZnO}$ nanowires allowed the fabrication of thicker active polymer layers, and resulted in higher efficiency [485]. The polymers could be functionalized to give chemical bonds with the $\mathrm{ZnO}$ nanowire surface [486]. The band diagram of this hybrid structure is illustrated in Fig. 61(b) [482]. Upon light radiation, excitons were generated and dissociated at the interface between the electron donor P3HT and acceptor PCBM. The interface area between $\mathrm{ZnO}$ nanowires and $\mathrm{P} 3 \mathrm{HT}$ is much smaller than that between the two polymers, so exciton dissociation by the $\mathrm{ZnO}$ nanowires is less likely to happen in this case. The $\mathrm{VO}_{x}$ layer performed as an electron-blocking layer that obstructed the electron transport from $\mathrm{P} 3 \mathrm{HT}$ to the Ag electrode, which was found to be crucial for high device efficiency, as illustrated in Fig. 61(c) [482].

The reported efficiency was about $2 \%$, which is probably restricted by the following factors [481]: first was the low open circuit voltage that is defined by the difference between the donor HOMO level and the acceptor LUMO level, which was suggested to be due to the blocking effect of the $\mathrm{ZnO}$ layers on hole transport from the polymer to the ITO electrode [485]; Second was the $\mathrm{ZnO}$ nanowire spacing $(\sim 100 \mathrm{~nm})$, which is larger than the typical carrier diffusion length in P3HT $(<10 \mathrm{~nm})$, and this markedly decreased the short circuit current [481]. Control experiments were carried out without PCBM, and the short circuit 


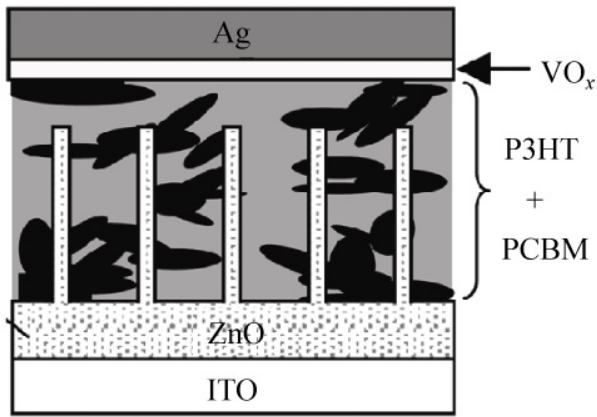

(a)

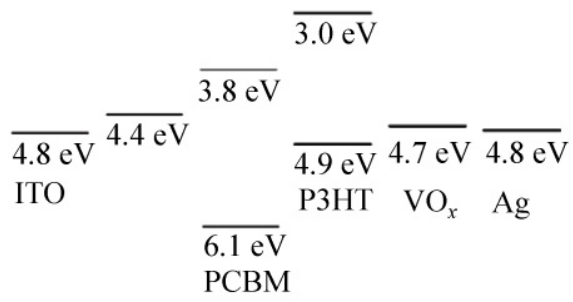

(b)

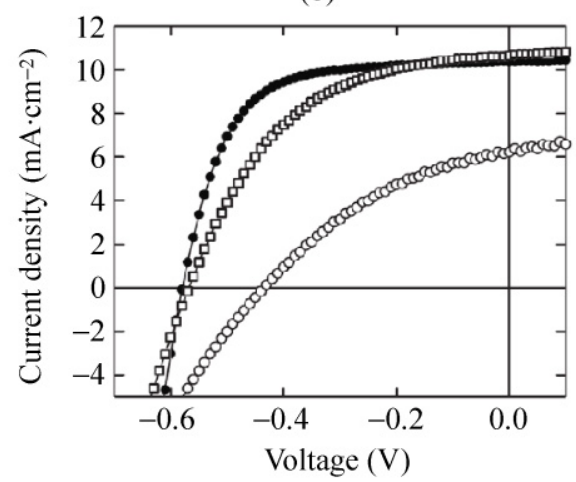

(c)

Figure 61 (a) Schematic structure and (b) corresponding energy diagram of a hybrid solar cell. (c) Current density-voltage characteristics of three differently configured solar cells. The open circles indicate the device with a $\mathrm{ZnO}$ thin film without a $\mathrm{VO}_{x}$ buffer layer, the open squares denote the device with a $\mathrm{VO}_{x}$ buffer layer, and the closed circles denote the device with $\mathrm{ZnO}$ nanowire arrays and $\mathrm{VO}_{x}$ buffer layer [482]. Reproduced with permission

current of the $\mathrm{ZnO} / \mathrm{P} 3 \mathrm{HT}$ solar cell decreased to only one fifth of that of the solar cell with PCBM. This is probably due to the large band offset between P3HT and $\mathrm{ZnO}$, which leads to inefficient exciton dissociation at the $\mathrm{ZnO} / \mathrm{P} 3 \mathrm{HT}$ interface. The PCBM probably serves as an offset stepping layer that enhances effective carrier transport. Similar studies have also been reported on an amphiphilic grafted dye molecule that not only bridged between hydrophilic $\mathrm{ZnO}$ nanowires and hydrophobic polymers, but also assisted interfacial electron cascade transportation by enhancing the electronic coupling between the polymer LUMO and the $\mathrm{ZnO}$ conduction band [487, 488]; Third is that the processing parameters such as the polymer infiltration, the solvent used, and the annealing temperature/ atmosphere were not optimized [489-491]. In an attempt to further enhance the efficiency, $\mathrm{ZnO} / \mathrm{TiO}_{2}$ heterojunctions have also been used in hybrid solar cells to facilitate the charge separation and transport [492, 493].

Finally, we note that since hybrid solar cells and hybrid LEDs share a similar structure, dual-functionality could be realized in a single device [494].

\subsubsection{Piezoelectric nanogenerators}

The search for sustainable micro/nano-power sources for driving wireless and mobile electronics is an emerging field in today's energy research, and could offer a fundamental solution to the energy needs of nanodevices/nanosystems [495-498]. A piezoelectric nanogenerator that converts mechanical energy into electricity was first demonstrated using $\mathrm{ZnO}$ nanowire arrays [22]. Following this initial discovery, both direct current $[23,499,500]$ and alternating current [24] $\mathrm{ZnO}$ nanogenerators have been developed. In addition, nanogenerators based on other materials, such as $\mathrm{GaN}$ nanowires [501-503], InN nanowires [504], AlGaN nanocones [505], CdS nanowires [506], $\mathrm{ZnO} / \mathrm{ZnS}$ heterojunction nanowires [507], lead zircornia titanates (PZT) nanofibers [508], nanoribbons [509-513], nanotubes [514], and single crystalline nanowires [515], $\mathrm{BaTiO}_{3}$ nanowires [516], $\mathrm{NaNbO}_{3}$ nanowires [517], and poly(vinylidene fluoride) (PVDF) nanofibers [518, 519], have shown promising potential for enhancing nanogenerator performance. Consequently, a worldwide effort has been launched in this regard, forming a new research field in nanotechnology and energy science [520].

\subsubsection{Fundamentals}

The non-centrosymmetry of the wurtzite $\mathrm{ZnO}$ crystal structure leads to piezoelectricity which arises from atomic scale polarization [25, 26, 521]. As shown in Fig. 62(a), the $\mathrm{ZnO}$ unit cell has a $\mathrm{Zn}^{2+}$ cation situated inside a tetrahedral cage formed by $\mathrm{O}^{2-}$ anions. Under non-stress conditions, the positive and negative centers of mass coincide with each other so there are no 
dipoles (Fig. 62(b)). When the crystal is subjected to a mechanical force, however, the positive and negative centers of mass become displaced with respect to each other, leading to an electric dipole (Fig. 62(c)). In single crystal $\mathrm{ZnO}$, electric dipoles in all the unit cells are lined up in the same direction, resulting in a macroscopic dipole with positive and negative charges built up on opposite crystal faces. This provides the basis for the nanogenerators.

Piezoelectric $\mathrm{ZnO}$ converts mechanical signals into electric signals, and vice versa. The polarization/surface charge density and applied stress are linearly related as shown by the equation: $P_{i}=d_{i j} \cdot T_{j}$, where $T_{j}$ is the mechanical stress exerted along direction $j, P_{i}$ is the induced polarization along direction $i$, and $d_{i j}$ is the piezoelectric coefficient. The strongest piezoelectric coefficient $d_{33}$ in $\mathrm{ZnO}$ arises from stress applied along the [0001] direction resulting in polarization in the [0001] direction as well. The effective piezoelectric coefficient $d_{33}$ of a $\mathrm{ZnO}$ nanowire/nanobelt grown by a vapor phase method was experimentally measured by piezoresponse force microscopy, and ranged from $14.3 \mathrm{pm} / \mathrm{V}$ to $26.7 \mathrm{pm} / \mathrm{V}$ depending on the frequency, which is higher than the value for bulk $\mathrm{ZnO}$ of $9.93 \mathrm{pm} / \mathrm{V}$ [522, 523], due to surface relaxation-induced volume reduction in nanowires/nanobelts-as shown by first principles calculations [523-526]. The converse piezoelectric effect also exists according to the

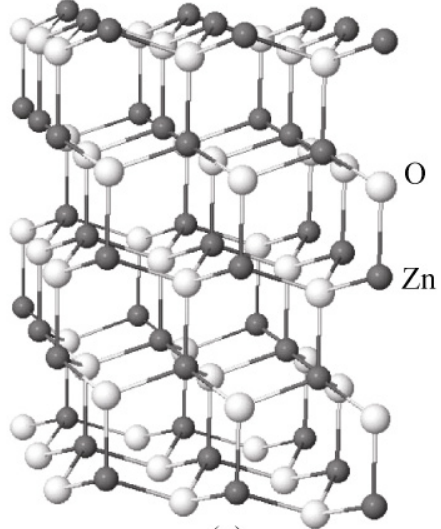

(a)

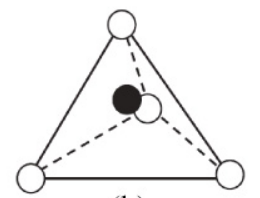

(b)

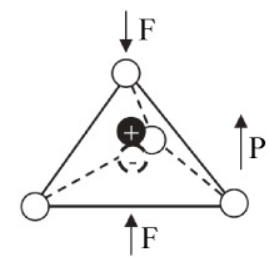

(c)
Figure 62 (a) Wurtzite $\mathrm{ZnO}$ showing the alternating polar surfaces along the [0001] direction. (b) Tetrahedral coordination between Zn (filled circles) and oxygen (open circles). (c) Distortion of the tetrahedral unit under compressive strain, showing the displacement of the positive charge center from the negative charge center [26]. Reproduced with permission equation: $S_{j}=d_{i j} \cdot E_{i}$, where $E_{i}$ is the applied electric field along direction $i$, and $S_{j}$ is the induced strain along direction $j$.

The advantage of using $\mathrm{ZnO}$ nanowires for mechanical energy harvesting is their robustness under high stress and responsiveness to tiny random disturbances/stimuli. Under elastic deformation, the nanowire builds up a piezoelectric potential field inside itself. The static ionic charges cannot freely move around. But in practice there are free electrons in intrinsic $n$-type $\mathrm{ZnO}$ nanowires due to the oxygen vacancies $\left(\mathrm{O}_{\mathrm{O}}^{x} \longleftrightarrow \frac{1}{2} \mathrm{O}_{2}(\mathrm{~g})+\mathrm{V}_{\mathrm{O}}^{\bullet \bullet}+2 \mathrm{e}^{\prime}\right)$ and zinc interstitials $\left(\mathrm{Zn}_{i}^{x} \longleftrightarrow \mathrm{Zn}_{i}^{\bullet \bullet}+2 \mathrm{e}^{\prime}\right)$. The free electrons may screen the positive piezoelectric charges to a certain degree. Within an elastic linear mechanics regime, a $\mathrm{ZnO}$ nanowire $50 \mathrm{~nm}$ in diameter and $500 \mathrm{~nm}$ in length is calculated to produce a piezoelectric potential of about $0.6 \mathrm{~V}$ if the free electrons inside are ignored [527]. The piezoelectric potential drops to $\sim 0.3 \mathrm{~V}$ due to the screening of the Madelung constant [528] if a moderate carrier concentration of $\sim 10^{16}-10^{17} \mathrm{~cm}^{-3}$ is taken into consideration [529]. The free carrier concentration in the nanowire is highly dependent on the synthesis and post-annealing conditions [126, 530], and may vary from one nanowire to another even from the same synthesis batch [528]. The finite piezoelectric potential indicates that the free electrons cannot completely neutralize or screen the piezoelectric ionic charge, even though it is reduced in magnitude. A potential of $0.3 \mathrm{~V}$ is sufficient to drive a nanogenerator and give useful work.

\subsubsection{Atomic force microscopy-based nanogenerators}

Nanogenerators are based on the coupling of piezoelectric and semiconducting properties. The first nanogenerator based on vertical $\mathrm{ZnO}$ nanowire arrays was demonstrated by Wang et al. using atomic force microscopy (AFM) [22]. $\mathrm{ZnO}$ nanowire arrays were grown by vapor phase transportation. As shown in Fig. 63(a), when a nanowire with its $c$ axis pointing up was scanned by a Pt-coated AFM tip in contact mode, a constant normal force of $\sim 5 \mathrm{nN}$ was maintained between the tip and the sample surface. The nanowire underwent deformation, and as a result created a strain field and charge separation across the 
nanowire with the tensile side positive and compressive side negative, as calculated by the strain and polarization relationship [531]. The central axis of the nanowire remained neutral. In addition to $\mathrm{Pt}, \mathrm{ZnO}$ has been reported to form Schottky diodes with a variety of metals, such as Ag [532], Au [533], Pd [534], and Ni [534]. Surface treatment can effectively improve the diode performance $[535,536]$. The Pt coated tip was grounded and had a zero potential. When the tip was in contact with the tensile side of the nanowire, the tip potential was lower than that of the $\mathrm{ZnO}$ nanowire. The Schottky diode was revered biased, and there was little current flowing through the tip/nanowire interface. When tip was scanned over the nanowire, there was a moment when it touched the compressive side of the nanowire. The Schottky barrier was then forward biased, and there was a relatively large amount of current flowing though the interface from the tip to the nanowire, which is detected as an electric pulse. The surface potential of a bent $\mathrm{ZnO}$ nanowire was measured to be in the range of $50 \mathrm{mV}$ to $80 \mathrm{mV}$ by Kelvin probe force microscopy [250]. Tip abrasion after scanning for long times reduced the magnitude of the output signals [537, 538], and this could be mitigated by diamond coating [539].

Following this working principle, Gao et al. demonstrated a flexible nanogenerator based on $\mathrm{ZnO}$ nanowire arrays on a conductive plastic substrate prepared by a wet chemical method [114]. The voltage generated from a single nanowire was as high as $45 \mathrm{mV}$. The AFM topography image and the corresponding output voltage profile from the $\mathrm{ZnO}$ nanowire array are shown in Figs. 63(b) and 63(c), respectively. By comparing the number of topographic peaks and electric pulses, the percentage of discharge events of the nanowires captured by the AFM tip was found to be about $60 \%$. By virtue of the flexibility of the device, very large deformations could be applied to both the nanowires and the substrate, indicating the potential of the device to act as a power source for flexible and foldable electronics.

Similarly, a nanotube-based nanogenerator has also been demonstrated [177]. It was shown that $\mathrm{ZnO}$ nanotubes were approximately five times more flexible than the corresponding nanowires, which leads to enhanced output voltage in nanogenerators [540].

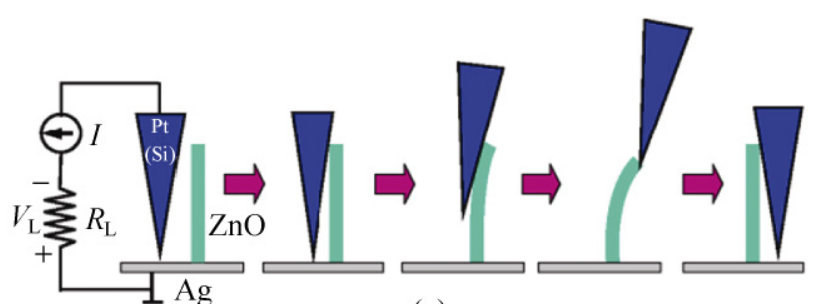

(a)

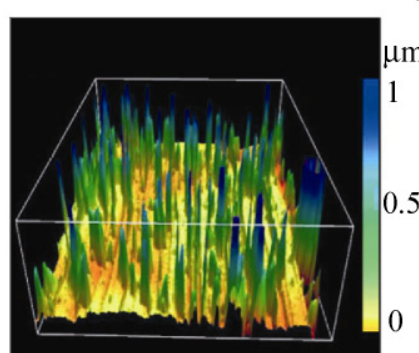

(b)

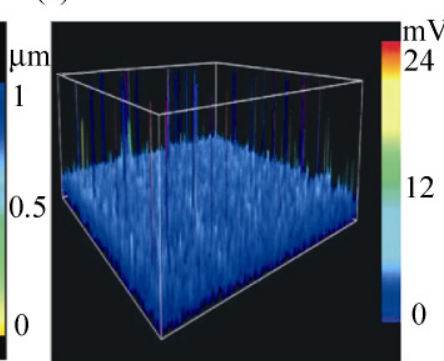

(c)
Figure 63 (a) Experimental setup and working procedure of the nanogenerator based on AFM [22]. (b) The AFM topography image and (c) the corresponding voltage output profile obtained over a $40 \mu \mathrm{m} \times 40 \mu \mathrm{m}$ area of vertically aligned $\mathrm{ZnO}$ nanowires [114]. Reproduced with permission

\subsubsection{Direct current nanogenerators}

To improve the power generation capabilities of a nanogenerator, it is necessary to replace the AFM tip with a simpler source of mechanical energy that can actuate an array of nanowires simultaneously and continuously. Wang et al. designed direct current nanogenerators [23]. Basically, they replaced the AFM tip with an array of zigzag shaped teeth as the top electrode. By covering the zigzag top electrode over an array of $\mathrm{ZnO}$ nanowires, encapsulating the entire structure with flexible polymers, and then exciting the device with ultrasonic waves, a constant and steady d.c. output was observed. In this design, the zigzag top electrode mimicked an array of AFM tips. When excited by ultrasonic waves, there is a relative movement between the top electrode and the free standing $\mathrm{ZnO}$ nanowires. The ultrasonic waves can also vibrate the nanowires. Each and every zigzag tooth on the top electrode performed as an AFM tip to bend and collect the electrical discharges from the nanowires underneath. Due to the rectification of the Schottky diode, the discharge current of each pair of tooth and nanowire was in the same direction, going from the tip to the nanowire. The resulting millions of electric pulses added together, give rise to a continuous direct current output [23]. 
The weakness with this design, however, is that only a small fraction of the nanowires are actually active in the current generating process, because most of them are either too short to reach the zigzag electrode, or not at the right position to be bent by the zigzag top electrode. Due to the non-uniform heights and random distribution of the nanowires, it was estimated that only $5 \%$ of the nanowires were actually active in the current generation process, while the other nanowires performed as parasitic capacitors bringing down the overall output voltage [541].

In order to improve the device performance, there are two straightforward approaches to increase the number of active nanowires. First, the distribution of the $\mathrm{ZnO}$ nanowires on the substrate needs to be controlled [48], so that it matches the shape and morphology of the zigzag top electrode. This will eventually enlarge the portion of active nanowires which can generate electricity. The other approach is to design a nanogenerator that is composed of paired nanotip-to-nanowire nanobrushes as illustrated in Fig. 64(a) [100]. This configuration can be achieved on flat substrates [100], or on curved fibers [96, 542]. Both the $\mathrm{ZnO}$ nanotip and nanowire arrays could be synthesized by wet chemical methods at $<100{ }^{\circ} \mathrm{C}$ on the two surfaces of a common substrate (Figs. 64(b) and $64(\mathrm{c})$ ). The densities of the $\mathrm{ZnO}$ nanowires and nanotips play a critical role in the current generating process. If they are too high, when the nanowire is bent, the nanowire will probably touch the adjacent nanowires, which will result in charge leakage and decrease the voltage output of the nanogenerator. If the density is too low, then the output power density will be rather small. The Au-coated nanotip array acted like the AFM tip array, partially interpenetrating into the spacing between the nanowires underneath, just like two face-to-face brushes with a small degree of interdigitated overlap at the two ends. In the same way, a multilayered nanogenerator was integrated by stacking multiple pieces of such structures together with each layer having two terminals. As shown in Fig. 64(d), four layers of nanogenerators, L1, L2, L3, and L4, had output voltage of $11 \mathrm{mV}, 14 \mathrm{mV}, 16 \mathrm{mV}$, and $20 \mathrm{mV}$, respectively. When they were connected in series, the resultant total output voltage was $\sim 62 \mathrm{mV}$, which is approximately their numerical sum. The output power density reached about $0.11 \mu \mathrm{W} / \mathrm{cm}^{2}$.

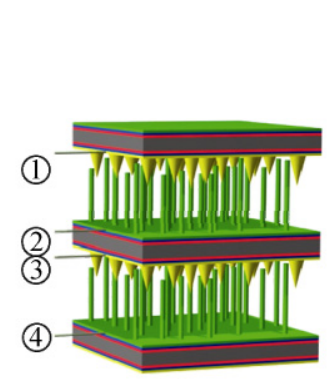

(a)

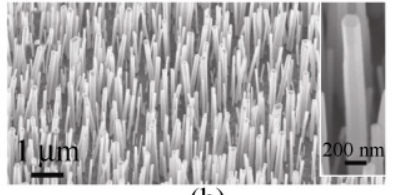

(b)

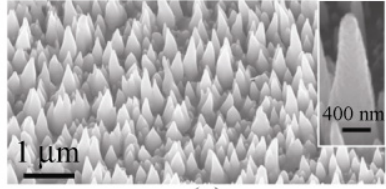

(c)

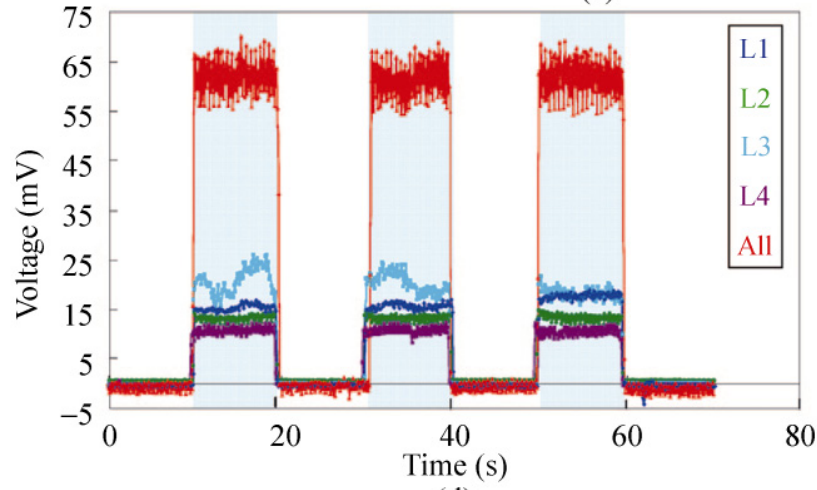

(d)
Figure 64 (a) Basic design of a two-layered nanogenerator. SEM images of the rational growth of (b) $\mathrm{ZnO}$ nanowire and (c) $\mathrm{ZnO}$ nanotip arrays by wet chemical methods. (d) Nanogenerator output voltages measured from four individual layers and the four-layer device connected in series. The stimulating ultrasonic wave was turned on and off periodically with an interval of $10 \mathrm{~s}$ [100]. Reproduced with permission

This nanotip-to-nanowire approach has four major advantages. First, both the nanotip and the nanowire arrays are randomly distributed on the substrate. Therefore, it is not necessary to pay extra effort to define the nanostructure positions on the substrate, which simplifies the fabrication process and will bring down the cost when scaling up. Second, the heights of both nanotips and nanowires are not uniform so that long nanowires can be covered by short nanotips, and short nanowires can be reached by long nanotips. Third, the nanotip and nanowire arrays can be grown on both sides of a substrate, which affords a layer-bylayer stacking of multiple electrodes. The output voltage and current can therefore be greatly enhanced by simply connecting these electrodes in series or in parallel. Fourth, the operation of the nanogenerator relies on mechanical deflection/bending of the nanowires, and resonance of nanowires is not required. This significantly expands the applications of the nanogenerator from low frequencies (approximately 
the hertz range) to relatively high frequencies (approximately the megahertz range), and allows effective harvesting of mechanical energies in our everyday life $[100,543]$.

$\mathrm{ZnO}$ nanowire arrays are optically transparent [89]. Therefore, by replacing the Si wafers with flexible and transparent conducting electrodes, such as ITO deposited on plastic substrates [499, 544], carbon nanotube networks [545], and graphene [546-548], rollable and transparent nanogenerators can be fabricated as power sources for touch sensors, artificial skins, and wearable personal electronics.

\subsubsection{Alternating current nanogenerators}

Yang et al. reported a flexible alternating current generator based on cyclic stretching/releasing of a $\mathrm{ZnO}$ fine wire that was firmly attached to metal electrodes at both ends [24, 549]. Low frequency cyclic stretching and releasing of a single $\mathrm{ZnO}$ wire with a strain of $0.05 \%-0.1 \%$ created an alternating output voltage of up to $\sim 50 \mathrm{mV}$ and an output current of a fraction of a nanoampere, with an energy conversion efficiency of about $6.8 \%$. The working principle from the energy band diagram point of view is illustrated in Fig. 65. There is a Schottky diode on one side of the wire. The Fermi levels of both sides are lined up when the wire is free (Fig. 65(a)). When the wire is subjected to tensile strain, a piezoelectric field is created in the wire with the left side positive and right side negative if the $c$ axis of the wire is to the left (Fig. 65(b)). Then the Fermi

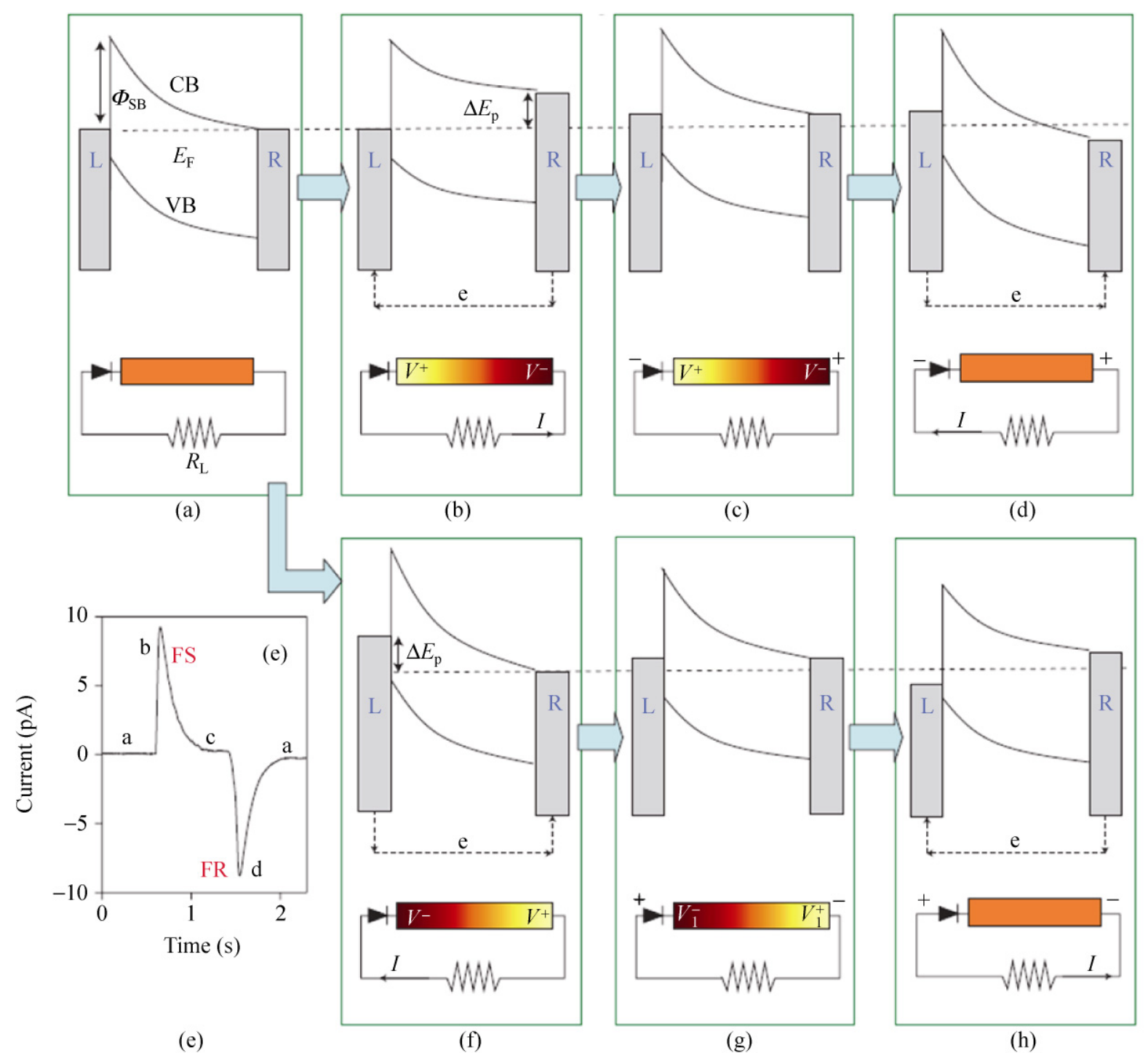

Figure 65 Proposed working mechanism for a single $\mathrm{ZnO}$ wire alternating current generator [24]. Reproduced with permission 
level of the right electrode is raised up with respect to the left electrode, which drives the electrons flow from the right electrode to the left electrode through an external load. The electrons do not pass through but rather accumulate at the wire/electrode interface because there is a Schottky barrier there (Fig. 65(c)). These accumulated electrons gradually rise up to the Fermi level of the left electrode until the Fermi levels of two electrodes reach a new equilibrium (Fig. 65(c)). When the tensile strain in the wire is released, the piezoelectric potential disappears, which lowers the Fermi level of the right electrode. Then the electrons accumulated at the wire/left electrode interface flow back through the external circuit to the right electrode (Fig. 65(d)), resetting the system to its original state. The output electric signals corresponding to this process are shown in Fig. 65(e). The same analysis applies when the wire is under compressive strain, or the $c$ axis of the wire is to the right (Fig. 65(f) and 65(g)) [24]. This design can effectively harvest low frequency mechanical energy in the environment $[550,551]$.

The voltage and power produced by a single wire are insufficient for real devices. The integration of large numbers of nanowire energy harvesters into a single power source is therefore necessary [432, 552]. Using the same principle, an integration of 700 rows of $\mathrm{ZnO}$ nanowires on a flexible substrate has been demonstrated, with each row containing $\sim 20,000$ nanowires, as shown in Fig. 66 [432]. When the substrate was stretched, all the nanowires on a substrate were subject to a pure tensile strain because the diameter of a nanowire is much smaller than the thickness of the substrate. Each active nanowire works as a "charging pump", and is independent of the other nanowires as the substrate is bent and released. The integrated nanogenerator was capable of producing a peak voltage of $1.26 \mathrm{~V}$ at a low strain of $0.19 \%$, which is sufficient to recharge an AA battery. Integration of so many nanowires has two key requirements. First, all the nanowires should have the same crystallographic orientation to ensure the polarities of the generated piezoelectric potentials are aligned. The nanowires therefore need to be rationally grown, directly on the substrate, rather than by chemical assembly, which usually gives orientational alignment but not alignment of crystallographic polarity. Second, the charging and

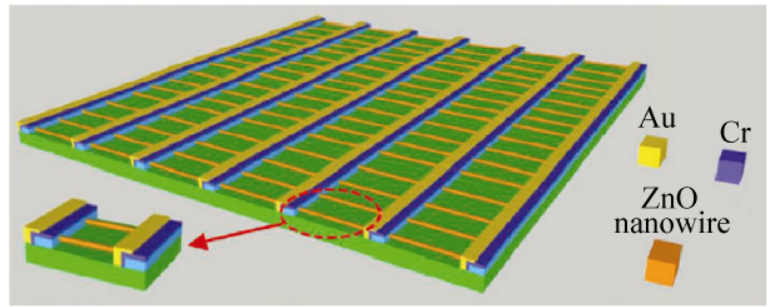

(a)

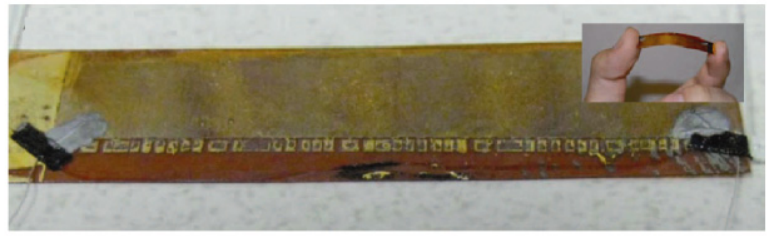

(b)

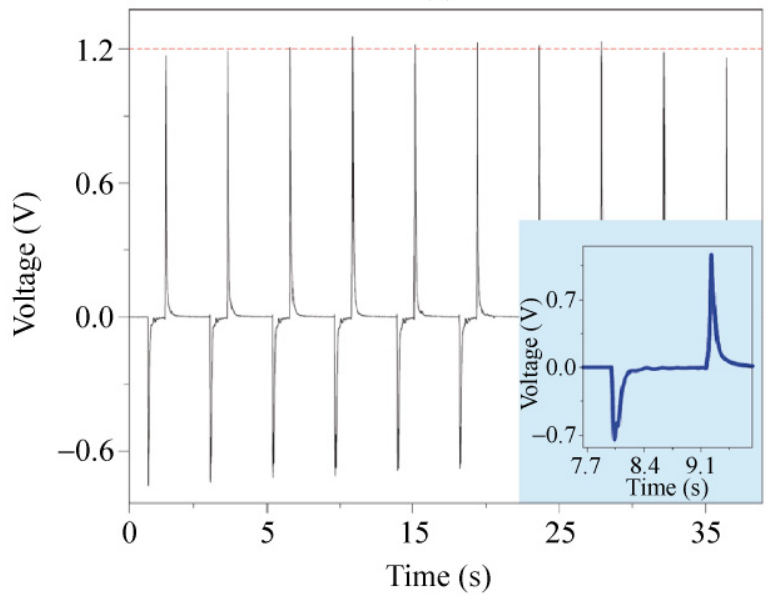

(c)

Figure 66 (a) Schematic design of a laterally integrated nanowire array nanogenerator, in which gold and chromium are used to create Schottky and ohmic contacts at the two ends of the lateral nanowires, respectively. (b) Low-magnification optical image of the laterally integrated nanogenerator. Inset in (b) is a demonstration of the nanogenerator flexibility. (c) Open circuit output voltage measured for a laterally integrated nanogenerator composed of 700 rows of nanowire arrays. The inset in (c) is the output voltage for one cycle of mechanical deformation. The laterally integrated nanogenerator was periodically deformed at a straining rate of $2.13 \% \mathrm{~s}^{-1}$ to a maximum strain of $0.19 \%$ [432]. Reproduced with permission

discharging processes of the nanowires should be synchronized so that the output alternating voltages can be added constructively, resulting in a high output voltage. It is interesting that the output voltage was greatly enhanced by lateral integration, but the output current was rather limited $(\sim 26 \mathrm{nA})$, which is probably attributable to the following factors. First, the orientational alignment of the as-grown lateral nanowires was not perfect and only a fraction of them were in reality in contact with the gold electrode. Of 
the nanowires that were in contact, only a fraction of these were actually actively outputting electricity, and the inactive nanowires acted as a capacitance to reduce the output current and voltage. Second, the binding between the gold and $\mathrm{ZnO}$ was not very strong, and could become loosened during repeated mechanical stretching cycles. According to theoretical calculations, within the elastic linear mechanics regime, the output voltage of a single nanowire is linearly proportional to the magnitude of its deformation [527]. A maximum strain of only $0.19 \%$ was applied in this study, which is much smaller than the $6 \%$ maximum tensile strain predicted theoretically for a $\mathrm{ZnO}$ nanowire before fracture [553]. Finally, the inner resistance of the entire integrated sheet was 1-10 M $\Omega$, which significantly reduced the total output current.

As an alternative to the lateral configuration, vertically aligned $\mathrm{ZnO}$ nanowire arrays can also be utilized to fabricate alternating current nanogenerators based on the same working principle. All of the nanowires are connected in parallel, and every nanowire works as a charge pump, independent from one another, as shown in Fig. 67(a) and 67(b) [432]. The nanowires were grown on a flat gold-coated $\mathrm{Si}$ wafer by a wet chemical method. The crystallographic alignment of the nanowires indicates their piezoelectric alignment in response to the external stress. Thus, the piezoelectric potentials created along each nanowire had the same tendency of distribution, leading to an enhanced macroscopic behavior. The presence of a Schottky contact at least at one end of the nanowires is essential for the operation of the vertically integrated alternating nanogenerator. The output voltage could be greatly enhanced by linearly integrating a number of layers of vertically integrated alternating nanogenerators [432, 554]. As shown in Fig. 67(c), three vertically integrated alternating nanogenerators with individual output voltages of 80, 90, and $96 \mathrm{mV}$, respectively, were connected in series, leading to an output voltage of $0.243 \mathrm{~V}$. The output current could also be enhanced by parallel stacking several layers of vertically integrated alternating nanogenerators [432].

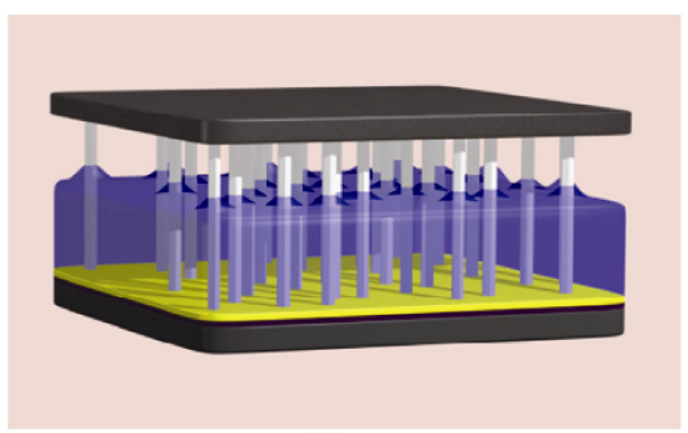

(a)

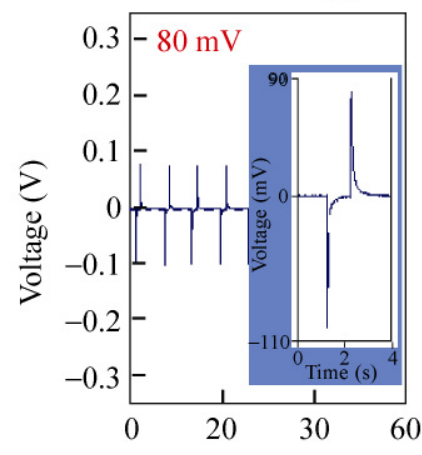

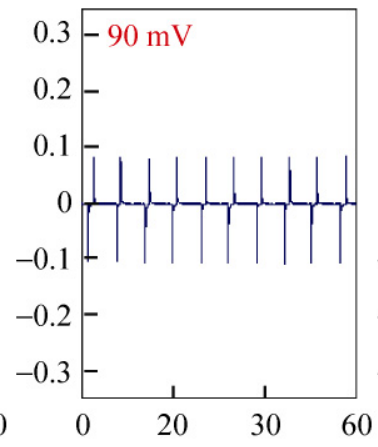

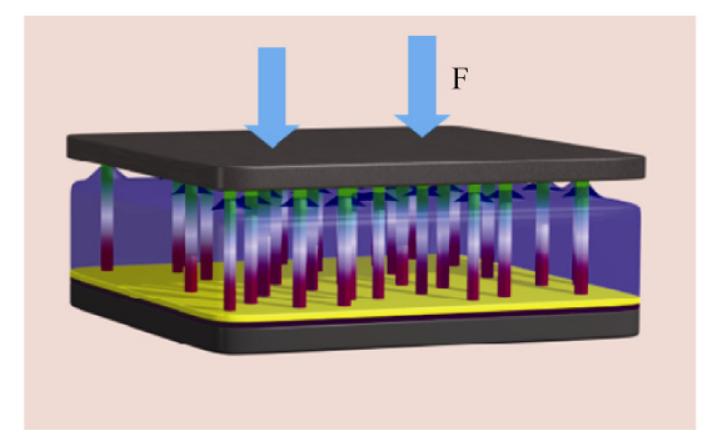

(b)
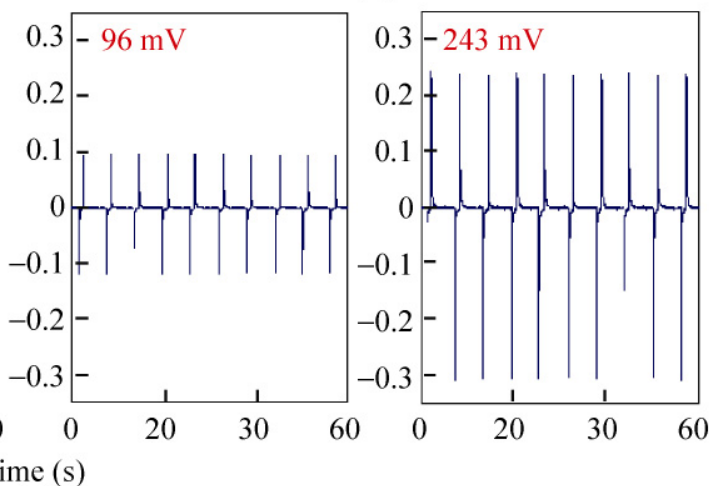

(c)

Figure 67 (a) Schematic structure of a vertically integrated alternating current nanogenerator, and (b) the nanogenerator under uniaxial strain. (c) Enhancing the output voltage of the nanogenerator by integrating them in series. Inset in the left panel is the an enlarged view of a single pulse [432]. Reproduced with permission 


\subsubsection{Self-powered nanosystems}

Building self-powered nanosystems is a future direction of nanotechnology [495]. A nanosystem is an integration of nanodevices, functional components, and a power source. Energy harvesting from the environment for powering a nanosystem is vitally important for its independent, wireless, and sustainable operation [495-497, 555]. A piezoelectric nanogenerator is a promising approach for this application [22].

A "self-powered" nanosystem has been demonstrated based on a vertical $\mathrm{ZnO}$ nanowire array nanogenerator and a single nanowire-based nanosensor. The two separate components were connected in series to form a loop. As shown in the inset in Fig. 68, a vertical nanowire array nanogenerator was connected to a $\mathrm{ZnO}$ nanowire-based $\mathrm{pH}$ sensor, and the voltage across the nanosensor was monitored by a voltmeter. The $\mathrm{pH}$ sensor was coated with a $10 \mathrm{~nm} \mathrm{Si}{ }_{3} \mathrm{~N}_{4}$ layer, which was thin enough to allow electrostatic interaction between the surface adsorbed charges and the carriers in the nanowire. By powering the $\mathrm{pH}$ sensor using a vertical nanowire array nanogenerator that generated an output voltage of $\sim 40 \mathrm{mV}$, a clear sensitivity to local $\mathrm{pH}$ change was observed. When the buffer solution was basic, the surface of the nanosensor was dominated by $-\mathrm{O}^{-}$groups. These negatively charged groups resulted in depletion regions at the surface of the $n$-type $\mathrm{ZnO}$ nanowire, which

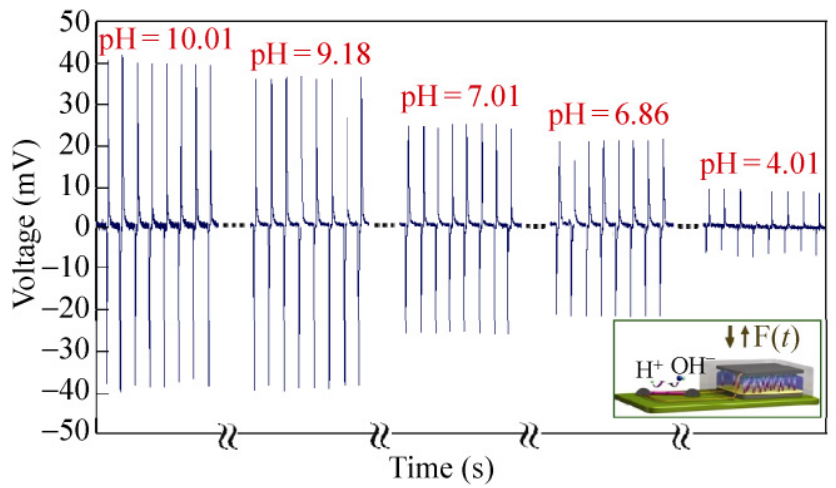

Figure 68 Voltage drop across a single $\mathrm{ZnO}$ nanowire-based $\mathrm{pH}$ sensor powered by a vertical nanogenerator with an output voltage of $40 \mathrm{mV}$, showing a stepwise drop of the voltage across the nanosensor as a function of its local $\mathrm{pH}$ value. The $\mathrm{ZnO}$ nanowire was covered with a thin layer of $\mathrm{Si}_{3} \mathrm{~N}_{4}$ and the testing was carried out within $1 \mathrm{~h}$ so that the etching effect from the solution was negligible [432]. Reproduced with permission increased the resistance of the $\mathrm{ZnO}$ nanowire. Thus, the voltage drop on the $\mathrm{ZnO}$ nanowire was relatively high. As the buffer solution was changed from basic to acidic, the surface of the nanosensor was gradually changed from $-\mathrm{O}^{-}$to $-\mathrm{OH}_{2}^{+}$groups. Therefore, the depletion regions at the $\mathrm{ZnO}$ nanowire surface diminished, which lowered the resistance of the $\mathrm{ZnO}$ nanowire. As the $\mathrm{pH}$ value of the testing buffer solution was changed from $10.01,9.18,7.01,6.86$, then to 4.01 , the voltage drop on the $\mathrm{pH}$ sensor changed accordingly.

This unambiguously indicated that the vertical nanowire array 20-40 $\mathrm{mV}$ nanogenerator could power a nanosensor. When using a variable resistor, the voltage across the resistor was found to be sensitive to the magnitude of its resistance and the result fits well with linear circuit theory [432]. The powering of a nanosensor was a pivotal step towards building self-powered, solely nanowire-based nanosystems. Recently, nanogenerators based on a $\mathrm{ZnO}$ nanowiretextured thin film were shown to produce an alternating output voltage of $10 \mathrm{~V}$, and after full wave charge rectification [515], wireless data transmission was realized [556].

\subsubsection{Water splitting}

Hydrogen has one of the highest energy density values. It combines with oxygen to produce electricity and water without generating pollutants. Semiconductor metal oxides, e.g., $\mathrm{ZnO}$ and $\mathrm{TiO}_{2}$ nanostructures, have been recognized for their potential as high performance photoanodes ( $n$-type) or photocathodes ( $p$-type) for water splitting in photoelectrochemical cells (PEC) [557]. In particular, $\mathrm{ZnO}$ nanowire arrays have been used for this purpose because of their large surface to volume ratio, appropriate direct band gap and flat band potential, low series resistance, and high electron transfer efficiency when compared with $\mathrm{TiO}_{2}$.

Yang et al. reported the implementation of $n$-type $\mathrm{ZnO}$ nanowire arrays as photoanodes in PEC cells for water splitting [311]. The nanowire arrays were grown by a hydrothermal method and were later annealed in ammonia to incorporate a controllable concentration of nitrogen atoms by varying the annealing time. The incorporation of nitrogen atoms into $\mathrm{ZnO}$ introduces intermediate energy levels in the $\mathrm{ZnO}$ band gap, 
which push the absorption spectrum of $\mathrm{ZnO}$ from the ultraviolet into the visible region, as evidenced by the enhanced incident photon to current efficiency in comparison with undoped $\mathrm{ZnO}$ nanowires. However, after nitrogen incorporation the nanowires were still n-type. CdTe-sensitized [558], CdS and CdSe quantum dot-cosensitized [559], and $\mathrm{ZnO-CdS}$ core-shell [560] nanowires have also been utilized to enhance the absorption in the visible region. It is believed that CdTe has a more favorable conduction band energy than CdSe and thus can inject electrons into $\mathrm{ZnO}$ more efficiently [558]. Also, quantum dots afford multiple excitons from a single absorbed photon, which can greatly increase the overall efficiency $[473,474]$. From Mott-Schottky measurements, the flat band potential, electron density, and depletion region thickness were determined to be $-0.58 \mathrm{~V}, 4.6 \times 10^{18} \mathrm{~cm}^{-3}$, and $22 \mathrm{~nm}$, respectively. Under illumination, electron and hole pairs were generated in the nanowire, and were separated in the depletion region at the nanowire surface, with holes drifting to the nanowire/electrolyte interface to oxidize the $\mathrm{OH}^{-}$groups and give rise to $\mathrm{O}_{2}$, while electrons flowed to the cathode and reduced the $\mathrm{H}^{+}$groups producing $\mathrm{H}_{2}$. The overall reaction for this water splitting process is:

$$
2 \mathrm{H}_{2} \mathrm{O}+\text { light } \longrightarrow 2 \mathrm{H}_{2}+\mathrm{O}_{2}
$$

The nitrogen-doped $\mathrm{ZnO}$ nanowires showed a phototo-hydrogen conversion efficiency of $0.15 \%$ under an external potential of $+0.5 \mathrm{~V}$ [311]. To improve the efficiency, $\mathrm{ZnO}$ nanowire/multi-wall carbon nanotube heterostructures were employed to increase the overall active surface area of the PEC device [247].

Non-ideal factors with PEC devices include the photodecomposition and dissolution of the anode itself - that is, oxidation of the anode by holes in the depletion region if the photopotential is higher than the redox potential of the anode material. Another major source of non-ideality is the presence of energy states associated with the electrode surface that are different from those in the interior of the electrode. These surface states can act as efficient channels for electron transfer, if they are at an appropriate level.

In addition to the PEC devices, Hong et al. showed that a piezoelectric potential could also be implemented to drive the nonspontaneous water splitting reaction [561]. The working principle is shown in Fig. 69. Under mechanical agitation by ultrasonic waves, $\mathrm{ZnO}$ nanowires, or any other piezoelectric materials, undergo physical deformation and generate surface charges on the tensile and compressive sides. When the piezopotential was greater than the standard redox potential of $\mathrm{H}_{2} \mathrm{O}(1.23 \mathrm{eV})$, then $\mathrm{H}_{2} \mathrm{O}$ can be split into $\mathrm{H}_{2}$ and $\mathrm{O}_{2}$ in the molar ratio of $2: 1$. This indicates that these piezoelectric nanostructures can scavenge mechanical energy waste from the environment, such as noise vibrations, to produce $\mathrm{H}_{2}$ for green energy applications.

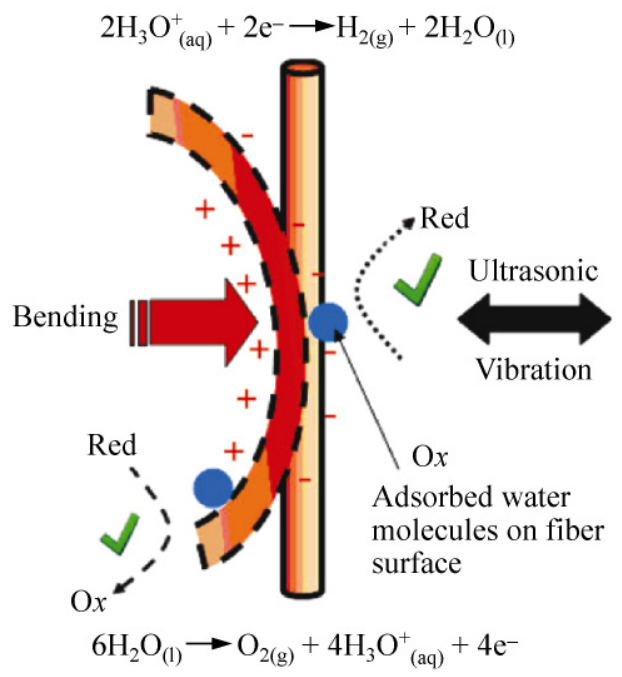

Figure 69 Schematic diagram showing that by bending under ultrasonic vibration, the charges and piezopotential developed on a piezoelectric material, such as a $\mathrm{ZnO}$ fiber, can drive the splitting of water into oxygen and hydrogen [561]. Reproduced with permission

\section{Concluding remarks}

Before reaching a conclusion, we would like to share our perspectives on some critical issues in the field and possible solutions to potentially address these issues.

There are three main challenges. The first is to control the crystal quality. $\mathrm{ZnO}$ nanostructures grown by wet chemical methods are usually of low crystal quality with abundant defects, such as point defects and voids, which can sometimes be seen from their very rough surfaces. These defects result in low fracture toughness, low carrier mobility, short carrier 
lifetime, and high threshold pump power, greatly undermining the mechanical, electrical, and optical properties of the nanostructures. The high defect level is due to the nature of solution growth at low temperature in a rather complex environment composed of many kinds of ions and molecules. To improve the crystal quality, it is of critical importance to start from an appropriate precursor, choose well-controlled reaction parameters, and possibly introduce appropriate capping agents and/or even control some seemingly trivial species, such as the counter ions in the $\mathrm{Zn}^{2+}$ salt precursor and the dissolved oxygen concentration. The second is to minimize the surface conductivity for piezoelectric applications. $\mathrm{ZnO}$ naturally exhibits $n$-type surface conductivity due to the loss of oxygen atoms to the ambient environment $\left(\mathrm{O}_{\mathrm{O}}^{x} \longleftrightarrow \frac{1}{2} \mathrm{O}_{2}(\mathrm{~g})+\mathrm{V}_{\mathrm{O}}^{\bullet \bullet}+2 \mathrm{e}^{\prime}\right)$. The thickness of the surface conductive region is calculated to be on the order of tens of nanometers. This finite concentration of electrons will partially screen the piezoelectric potential, which significantly weakens the performance of $\mathrm{ZnO}$-based piezoelectric devices. To reduce the oxygen deficiency, it is possible to push the defect equation to the left by providing an oxygen-rich environment, such as annealing under high oxygen partial pressure or implanting energetic oxygen atoms under oxygen plasma. Finally, based on the reduced intrinsic $n$-type conductivity, it is feasible but still challenging to dope $\mathrm{ZnO}$ with group $\mathrm{V}$ and/or group I element atoms to achieve $p$-type conductivity. The doping reaction of $\mathrm{ZnO}$ nanostructures with foreign atoms is typically entropically favorable but enthalpically unfavorable. So it is desirable to choose appropriate elements with similar ionic radii and electronegativities under well-controlled reaction conditions (pressure, temperature, and precursor concentration) to minimize the enthalpy change. Although there are debates about $p$-type doping in $\mathrm{ZnO}$ thin films, $p$-doping in $1 \mathrm{D} \mathrm{ZnO}$ nanostructures appears quite promising due to the absence of dislocations.

Nevertheless, this article has given a comprehensive review of the growth control and functional applications of $1 \mathrm{D} \mathrm{ZnO}$ nanostructures prepared by wet chemical approaches. The resulting $1 \mathrm{D} \mathrm{ZnO}$ nanostructures are biologically compatible and environmentally safe. They have multi-functional materials with semicon- ducting, optical, piezoelectric and other properties. More importantly and most distinctly, they can be grown using low temperature wet chemical approaches, so that they can be easily integrated with a wide range of materials and technologies. All of these advantages combine to make $1 \mathrm{D} \mathrm{ZnO}$ nanostructures unique building blocks for fabricating diverse novel devices.

\section{Acknowledgements}

We thank NSF, DARPA, and BES DOE for support. We also thank those authors and many of our collaborators who made contributions to the published data reviewed in the text here.

\section{References}

[1] Ozgur, U.; Alivov, Y. I.; Liu, C.; Teke, A.; Reshchikov, M. A.; Dogan, S.; Avrutin, V.; Cho, S. J.; Morkoc, H. A comprehensive review of $\mathrm{ZnO}$ materials and devices. $J$. Appl. Phys. 2005, 98, 041301 .

[2] Look, D. C. Recent advances in $\mathrm{ZnO}$ materials and devices. Mat. Sci. Eng. B-.Adv. 2001, 80, 383-387.

[3] Heo, Y. W.; Norton, D. P.; Tien, L. C.; Kwon, Y.; Kang, B. S.; Ren, F.; Pearton, S. J.; LaRoche, J. R. ZnO nanowire growth and devices. Mat. Sci. Eng. R 2004, 47, 147.

[4] Yi, G. C.; Wang, C. R.; Park, W. I. ZnO nanorods: Synthesis, characterization and applications. Semicond. Sci. Technol. 2005, 20, S22-S34.

[5] Wang, Z. L. Oxide nanobelts and nanowires - growth, properties and applications. J. Nanosci. Nanotechno 2008, 8 , 27-55.

[6] Wang, Z. L. Splendid one-dimensional nanostructures of zinc oxide: A new nanomaterial family for nanotechnology. ACS Nano 2008, 2, 1987-1992.

[7] Wang, Z. L. ZnO nanowire and nanobelt platform for nanotechnology. Mat. Sci. Eng. R 2009, 64, 33-71.

[8] Wang, Z. L. Ten years' venturing in $\mathrm{ZnO}$ nanostructures: From discovery to scientific understanding and to technology applications. Chinese Sci. Bull. 2009, 54, 4021-4034.

[9] Huang, M. H.; Mao, S.; Feick, H.; Yan, H. Q.; Wu, Y. Y.; Kind, H.; Weber, E.; Russo, R.; Yang, P. D. Room-temperature ultraviolet nanowire nanolasers. Science 2001, 292, 18971899.

[10] Govender, K.; Boyle, D. S.; O'Brien, P.; Binks, D.; West, D.; Coleman, D. Room-temperature lasing observed from $\mathrm{ZnO}$ nanocolumns grown by aqueous solution deposition. $A d v$. Mater. 2002, 14, 1221-1224. 
[11] Park, W. I.; Yi, G. C. Electroluminescence in $n$-ZnO nanorod arrays vertically grown on $p-\mathrm{GaN}$. Adv. Mater. 2004, 16, 87-90.

[12] Mao, D. S.; Wang, X.; Li, W.; Liu, X. H.; Li, Q.; Xu, J. F. Electron field emission from hydrogen-free amorphous carbon-coated ZnO tip array. J. Vac. Sci. Technol. B 2002, 20, 278-281.

[13] Zhu, Y. W.; Zhang, H. Z.; Sun, X. C.; Feng, S. Q.; Xu, J.; Zhao, Q.; Xiang, B.; Wang, R. M.; Yu, D. P. Efficient field emission from ZnO nanoneedle arrays. Appl. Phys. Lett. 2003, 83, 144-146.

[14] Wang, W. Z.; Zeng, B. Q.; Yang, J.; Poudel, B.; Huang, J. Y.; Naughton, M. J.; Ren, Z. F. Aligned ultralong ZnO nanobelts and their enhanced field emission. Adv. Mater. 2006, 18, 3275-3278.

[15] Wei, T. Y.; Yeh, P. H.; Lu, S. Y.; Wang, Z. L. Gigantic enhancement in sensitivity using Schottky contacted nanowire nanosensor. J. Am. Chem. Soc. 2009, 131, 17690-17695.

[16] Yeh, P. H.; Li, Z.; Wang, Z. L. Schottky-gated probe-free ZnO nanowire biosensor. Adv. Mater. 2009, 21, 4975-4978.

[17] Zhou, J.; Gu, Y. D.; Hu, Y. F.; Mai, W. J.; Yeh, P. H.; Bao, G.; Sood, A. K.; Polla, D. L.; Wang, Z. L. Gigantic enhancement in response and reset time of $\mathrm{ZnO}$ UV nanosensor by utilizing Schottky contact and surface functionalization. Appl. Phys. Lett. 2009, 94, 191103.

[18] Law, M.; Greene, L. E.; Johnson, J. C.; Saykally, R.; Yang, P. D. Nanowire dye-sensitized solar cells. Nat. Mater. 2005, 4, 455-459.

[19] Levy-Clement, C.; Tena-Zaera, R.; Ryan, M. A.; Katty, A.; Hodes, G. CdSe-sensitized $p$-CuSCN/nanowire $n-\mathrm{ZnO}$ heterojunctions. Adv. Mater. 2005, 17, 1512-1515.

[20] Weintraub, B.; Wei, Y. G.; Wang, Z. L. Optical fiber/ nanowire hybrid structures for efficient three-dimensional dye-sensitized solar cells. Angew. Chem. Int. Ed. 2009, 48, 8981-8985.

[21] Wei, Y. G.; Xu, C.; Xu, S.; Li, C.; Wu, W. Z.; Wang, Z. L. Planar waveguide-nanowire integrated three-dimensional dyesensitized solar cells. Nano Lett. 2010, 10, 2092-2096.

[22] Wang, Z. L.; Song, J. H. Piezoelectric nanogenerators based on zinc oxide nanowire arrays. Science 2006, 312, 242-246.

[23] Wang, X. D.; Song, J. H.; Liu, J.; Wang, Z. L. Direct-current nanogenerator driven by ultrasonic waves. Science 2007, 316, 102-105.

[24] Yang, R. S.; Qin, Y.; Dai, L. M.; Wang, Z. L. Power generation with laterally packaged piezoelectric fine wires. Nat. Nanotechnol. 2009, 4, 34-39.

[25] Wang, Z. L. The new field of nanopiezotronics. Mater. Today 2007, 10, 20-28.

[26] Wang, Z. L. Nanopiezotronics. Adv. Mater. 2007, 19, 889892.
[27] Wang, Z. L. Towards self-powered nanosystems: From nanogenerators to nanopiezotronics. Adv. Funct. Mater. 2008, 18, 3553-3567.

[28] Laudise, R. A.; Ballman, A. A. Hydrothermal synthesis of zinc oxide and zinc sulfide. J. Phys. Chem. 1960, 64, 688-691.

[29] Verges, M. A.; Mifsud, A.; Serna, C. J. Formation of rodlike zinc-oxide microcrystals in homogeneous solutions. $J$. Chem. Soc., Faraday Trans. 1990, 86, 959-963.

[30] Vayssieres, L.; Keis, K.; Lindquist, S. E.; Hagfeldt, A. Purpose-built anisotropic metal oxide material: 3D highly oriented microrod array of ZnO. J. Phys. Chem. B 2001, 105, 3350-3352.

[31] Pan, Z. W.; Dai, Z. R.; Wang, Z. L. Nanobelts of semiconducting oxides. Science 2001, 291, 1947-1949.

[32] Huang, M. H.; Wu, Y. Y.; Feick, H.; Tran, N.; Weber, E.; Yang, P. D. Catalytic growth of zinc oxide nanowires by vapor transport. Adv. Mater. 2001, 13, 113-116.

[33] Yao, B. D.; Chan, Y. F.; Wang, N. Formation of $\mathrm{ZnO}$ nanostructures by a simple way of thermal evaporation. Appl. Phys. Lett. 2002, 81, 757-759.

[34] Park, W. I.; Yi, G. C.; Kim, M. Y.; Pennycook, S. J. ZnO Nanoneedles grown vertically on $\mathrm{Si}$ substrates by non-catalytic vapor-phase epitaxy. Adv. Mater. 2002, 14, 1841-1843.

[35] Park, W. I.; Kim, D. H.; Jung, S. W.; Yi, G. C. Metalorganic vapor-phase epitaxial growth of vertically well-aligned $\mathrm{ZnO}$ nanorods. Appl. Phys. Lett. 2002, 80, 4232-4234.

[36] Yuan, H.; Zhang, Y. Preparation of well-aligned $\mathrm{ZnO}$ whiskers on glass substrate by atmospheric MOCVD. J. Cryst. Growth 2004, 263, 119-124.

[37] Heo, Y. W.; Varadarajan, V.; Kaufman, M.; Kim, K.; Norton, D. P.; Ren, F.; Fleming, P. H. Site-specific growth of $\mathrm{ZnO}$ nanorods using catalysis-driven molecular-beam epitaxy. Appl. Phys. Lett. 2002, 81, 3046-3048.

[38] Sun, Y.; Fuge, G. M.; Ashfold, M. N. R. Growth of aligned $\mathrm{ZnO}$ nanorod arrays by catalyst-free pulsed laser deposition methods. Chem. Phys. Lett. 2004, 396, 21-26.

[39] Hong, J. I.; Bae, J.; Wang, Z. L.; Snyder, R. L. Roomtemperature, texture-controlled growth of $\mathrm{ZnO}$ thin films and their application for growing aligned $\mathrm{ZnO}$ nanowire arrays. Nanotechnology 2009, 20, 085609.

[40] Chiou, W. T.; Wu, W. Y.; Ting, J. M. Growth of single crystal $\mathrm{ZnO}$ nanowires using sputter deposition. Diam. Relat. Mater. 2003, 12, 1841-1844.

[41] Xu, C. K.; Xu, G. D.; Liu, Y. K.; Wang, G. H. A simple and novel route for the preparation of $\mathrm{ZnO}$ nanorods. Solid State Commun. 2002, 122, 175-179.

[42] Lin, D. D.; Pan, W.; Wu, H. Morphological control of centimeter long aluminum-doped zinc oxide nanofibers prepared by electrospinning. J. Am. Ceram. Soc. 2007, 90, 
71-76.

[43] Lin, D.; Wu, H.; Pan, W. Photoswitches and memories assembled by electrospinning aluminum-doped zinc oxide single nanowires. Adv. Mater. 2007, 19, 3968-3972.

[44] Sui, X. M.; Shao, C. L.; Liu, Y. C. White-light emission of polyvinyl alcohol/ZnO hybrid nanofibers prepared by electrospinning. Appl. Phys. Lett. 2005, 87, 113115.

[45] Wu, J. J.; Wen, H. I.; Tseng, C. H.; Liu, S. C. Well-aligned $\mathrm{ZnO}$ nanorods via hydrogen treatment of $\mathrm{ZnO}$ films. $A d v$. Funct. Mater. 2004, 14, 806-810.

[46] Zhang, H.; Yang, D. R.; Ma, X. Y.; Du, N.; Wu, J. B.; Que, D. L. Straight and thin $\mathrm{ZnO}$ nanorods: Hectogram-scale synthesis at low temperature and cathodoluminescence. $J$. Phys. Chem. B 2006, 110, 827-830.

[47] Chang, P. C.; Lu, J. G. ZnO nanowire field-effect transistors. IEEE T. Electron Dev. 2008, 55, 2977-2987.

[48] Xu, S.; Wei, Y.; Kirkham, M.; Liu, J.; Mai, W.; Davidovic, D.; Snyder, R. L.; Wang, Z. L. Patterned growth of vertically aligned $\mathrm{ZnO}$ nanowire arrays on inorganic substrates at low temperature without catalyst. J. Am. Chem. Soc. 2008, 130, 14958-14959.

[49] Govender, K.; Boyle, D. S.; Kenway, P. B.; O'Brien, P. Understanding the factors that govern the deposition and morphology of thin films of $\mathrm{ZnO}$ from aqueous solution. $J$. Mater. Chem. 2004, 14, 2575-2591.

[50] Xu, S.; Adiga, N.; Ba, S.; Dasgupta, T.; Wu, C. F. J.; Wang, Z. L. Optimizing and improving the growth quality of $\mathrm{ZnO}$ nanowire arrays guided by statistical design of experiments. ACS Nano 2009, 3, 1803-1812.

[51] Pearton, S. J.; Norton, D. P.; Ip, K.; Heo, Y. W.; Steiner, T. Recent progress in processing and properties of $\mathrm{ZnO}$. Prog. Mater. Sci. 2005, 50, 293-340.

[52] Klingshirn, C. ZnO: From basics towards applications. Phys. Status Solidi B 2007, 244, 3027-3073.

[53] Schmidt-Mende, L.; MacManus-Driscoll, J. L. ZnOnanostructures, defects, and devices. Mater. Today 2007, $10,40-48$.

[54] Zang, J. F.; Li, C. M.; Cui, X. Q.; Wang, J. X.; Sun, X. W.; Dong, H.; Sun, C. Q. Tailoring zinc oxide nanowires for high performance amperometric glucose sensor. Electroanal. 2007, 19, 1008-1014.

[55] Baruah, S.; Dutta, J. pH-dependent growth of zinc oxide nanorods. J. Cryst. Growth 2009, 311, 2549-2554.

[56] Xu, S.; Shen, Y.; Ding, Y.; Wang, Z. L. Growth and transfer of monolithic horizontal $\mathrm{ZnO}$ nanowire superstructures onto flexible substrates. Adv. Funct. Mater. 2010, 20, 1493-1495.

[57] Li, W. J.; Shi, E. W.; Zhong, W. Z.; Yin, Z. W. Growth mechanism and growth habit of oxide crystals. J. Cryst. Growth 1999, 203, 186-196.
[58] Demianets, L. N.; Kostomarov, D. V.; Kuz'mina, I. P.; Pushko, S. V. Mechanism of growth of $\mathrm{ZnO}$ single crystals from hydrothermal alkali solutions. Crystallogr. Rep. 2002, 47, S86-S98.

[59] Liu, B.; Zeng, H. C. Room temperature solution synthesis of monodispersed single-crystalline $\mathrm{ZnO}$ nanorods and derived hierarchical nanostructures. Langmuir 2004, 20, 4196-4204.

[60] Viswanatha, R.; Amenitsch, H.; Sarma, D. D. Growth kinetics of $\mathrm{ZnO}$ nanocrystals: A few surprises. J. Am. Chem. Soc. 2007, 129, 4470-4475.

[61] Demianets, L. N.; Kostomarov, D. V. Mechanism of zinc oxide single crystal growth under hydrothermal conditions. Ann. Chim. Sci. Mat. 2001, 26, 193-198.

[62] Dem'yanets, L. N.; Kostomarov, D. V.; Kuz-mina, I. P. Chemistry and kinetics of $\mathrm{ZnO}$ growth from alkaline hydrothermal solutions. Inorg. Mater. 2002, 38, 124-131.

[63] Kawska, A.; Duchstein, P.; Hochrein, O.; Zahn, D. Atomistic mechanisms of $\mathrm{ZnO}$ aggregation from ethanolic solution: Ion association, proton transfer, and self-organization. Nano Lett. 2008, 8, 2336-2340.

[64] Yamabi, S.; Imai, H. Growth conditions for wurtzite zinc oxide films in aqueous solutions. J. Mater. Chem. 2002, 12, 3773-3778.

[65] Zhang, J.; Sun, L. D.; Yin, J. L.; Su, H. L.; Liao, C. S.; Yan, C. H. Control of $\mathrm{ZnO}$ morphology via a simple solution route. Chem. Mater. 2002, 14, 4172-4177.

[66] Cheng, B.; Samulski, E. T. Hydrothermal synthesis of onedimensional $\mathrm{ZnO}$ nanostructures with different aspect ratios. Chem. Commun. 2004, 986-987.

[67] Liu, B.; Zeng, H. C. Hydrothermal synthesis of $\mathrm{ZnO}$ nanorods in the diameter regime of $50 \mathrm{~nm}$. J. Am. Chem. Soc. 2003, 125, 4430-4431.

[68] Cao, H. L.; Qian, X. F.; Gong, Q.; Du, W. M.; Ma, X. D.; Zhu, Z. K. Shape- and size-controlled synthesis of nanometre $\mathrm{ZnO}$ from a simple solution route at room temperature. Nanotechnology 2006, 17, 3632-3636.

[69] Hou, X. M.; Zhou, F.; Sun, Y. B.; Liu, W. M. Ultrasoundassisted synthesis of dentritic $\mathrm{ZnO}$ nanostructure in ionic liquid. Mater. Lett. 2007, 61, 1789-1792.

[70] Alammar, T.; Mudring, A. V. Facile ultrasound-assisted synthesis of $\mathrm{ZnO}$ nanorods in an ionic liquid. Mater. Lett. 2009, 63, 732-735.

[71] Yin, M.; Gu, Y.; Kuskovsky, I. L.; Andelman, T.; Zhu, Y.; Neumark, G. F.; O'Brien, S. Zinc oxide quantum rods. $J$. Am. Chem. Soc. 2004, 126, 6206-6207.

[72] Pacholski, C.; Kornowski, A.; Weller, H. Self-assembly of $\mathrm{ZnO}$ : From nanodots to nanorods. Angew. Chem. Int. Ed. 2002, 41, 1188-1191.

[73] Zhang, D. F.; Sun, L. D.; Yin, J. L.; Yan, C. H.; Wang, R. M. 
Attachment-driven morphology evolvement of rectangular ZnO nanowires. J. Phys. Chem. B 2005, 109, 8786-8790.

[74] Guo, L.; Ji, Y. L.; Xu, H. B.; Simon, P.; Wu, Z. Y. Regularly shaped, single-crystalline $\mathrm{ZnO}$ nanorods with wurtzite structure. J. Am. Chem. Soc. 2002, 124, 14864 14865.

[75] Liu, J. P.; Huang, X. T.; Li, Y. Y.; Ji, X. X.; Li, Z. K.; He, X.; Sun, F. L. Vertically aligned $1 \mathrm{D} \mathrm{ZnO}$ nanostructures on bulk alloy substrates: Direct solution synthesis, photoluminescence, and field emission. J. Phys. Chem. C 2007, 111, 4990-4997.

[76] Gao, Y. F.; Nagai, M.; Chang, T. C.; Shyue, J. J. Solutionderived $\mathrm{ZnO}$ nanowire array film as photoelectrode in dyesensitized solar cells. Cryst. Growth Des. 2007, 7, 2467 2471.

[77] Tak, Y.; Yong, K. J. Controlled growth of well-aligned $\mathrm{ZnO}$ nanorod array using a novel solution method. J. Phys. Chem. B 2005, 109, 19263-19269.

[78] Xu, C. K.; Shin, P.; Cao, L. L.; Gao, D. Preferential growth of long $\mathrm{ZnO}$ nanowire array and its application in dye-sensitized solar cells. J. Phys. Chem. C 2010, 114, 125-129.

[79] Postels, B.; Wehmann, H. H.; Bakin, A.; Kreye, M.; Fuhrmann, D.; Blaesing, J.; Hangleiter, A.; Krost, A.; Waag, A. Controlled low-temperature fabrication of $\mathrm{ZnO}$ nanopillars with a wet-chemical approach. Nanotechnology 2007, 18, 195602.

[80] Hua, G. M.; Zhang, Y.; Zhang, J. X.; Cao, X. L.; Xu, W.; Zhang, L. D. Fabrication of $\mathrm{ZnO}$ nanowire arrays by cycle growth in surfactantless aqueous solution and their applications on dye-sensitized solar cells. Mater. Lett. 2008, 62, 41094111.

[81] Tang, Q.; Zhou, W. J.; Shen, J. M.; Zhang, W.; Kong, L. F.; Qian, Y. T. A template-free aqueous route to $\mathrm{ZnO}$ nanorod arrays with high optical property. Chem. Commun. 2004, 712-713.

[82] Xu, S.; Wang, Z. L. Unpublished results.

[83] Boyle, D. S.; Govender, K.; O'Brien, P. Novel low temperature solution deposition of perpendicularly orientated rods of $\mathrm{ZnO}$ : Substrate effects and evidence of the importance of counter-ions in the control of crystallite growth. Chem. Commun. 2002, 80-81.

[84] Vayssieres, L. Growth of arrayed nanorods and nanowires of $\mathrm{ZnO}$ from aqueous solutions. Adv. Mater. 2003, 15, 464- 466.

[85] Ahuja, I. S.; Yadava, C. L.; Singh, R. Structural information on manganese(II), cobalt(II), nickel(II), zinc(II) and cadmium (II) sulphate complexes with hexamethylenetetramine (a potentially tetradentate ligand) from their magnetic moments, electronic and infrared spectra. J. Mol. Struct. 1982, 81, 229-234.

[86] Baruah, S.; Dutta, J. Hydrothermal growth of $\mathrm{ZnO}$ nano- structures. Sci. Technol. Adv. Mat. 2009, 10, 013001.

[87] Ashfold, M. N. R.; Doherty, R. P.; Ndifor-Angwafor, N. G.; Riley, D. J.; Sun, Y. The kinetics of the hydrothermal growth of $\mathrm{ZnO}$ nanostructures. Thin Solid Films 2007, 515, 8679-8683.

[88] Unalan, H. E.; Hiralal, P.; Rupesinghe, N.; Dalal, S.; Milne, W. I.; Amaratunga, G. A. J. Rapid synthesis of aligned zinc oxide nanowires. Nanotechnology 2008, 19, 255608.

[89] Jung, S. H.; Oh, E.; Lee, K. H.; Park, W.; Jeong, S. H. A sonochemical method for fabricating aligned $\mathrm{ZnO}$ nanorods. Adv. Mater. 2007, 19, 749-753.

[90] Shi, L.; Bao, K. Y.; Cao, J.; Qian, Y. T. Sunlight-assisted fabrication of a hierarchical $\mathrm{ZnO}$ nanorod array structure. CrystEngComm 2009, 11, 2009-2014.

[91] Xu, S.; Lao, C.; Weintraub, B.; Wang, Z. L. Densitycontrolled growth of aligned $\mathrm{ZnO}$ nanowire arrays by seedless chemical approach on smooth surfaces. J. Mater. Res. 2008, 23, 2072-2077.

[92] Cheng, C. W.; Yan, B.; Wong, S. M.; Li, X. L.; Zhou, W. W.; Yu, T.; Shen, Z. X.; Yu, H. Y.; Fan, H. J. Fabrication and SERS performance of silver-nanoparticle-decorated $\mathrm{Si} / \mathrm{ZnO}$ nanotrees in ordered arrays. ACS Appl. Mater. Inter. 2010, 2, 1824-1828.

[93] Greene, L. E.; Law, M.; Goldberger, J.; Kim, F.; Johnson, J. C.; Zhang, Y. F.; Saykally, R. J.; Yang, P. D. Lowtemperature wafer-scale production of $\mathrm{ZnO}$ nanowire arrays. Angew. Chem. Int. Ed. 2003, 42, 3031-3034.

[94] Liu, T. Y.; Liao, H. C.; Lin, C. C.; Hu, S. H.; Chen, S. Y. Biofunctional $\mathrm{ZnO}$ nanorod arrays grown on flexible substrates. Langmuir 2006, 22, 5804-5809.

[95] Manekkathodi, A.; Lu, M. Y.; Wang, C. W.; Chen, L. J. Direct growth of aligned zinc oxide nanorods on paper substrates for low-cost flexible electronics. Adv. Mater. 2010, 22, 40594063.

[96] Qin, Y.; Wang, X. D.; Wang, Z. L. Microfibre-nanowire hybrid structure for energy scavenging. Nature 2008, 451, 809-813.

[97] Bae, J.; Song, M. K.; Park, Y. J.; Kim, J. M.; Liu, M. L.; Wang, Z. L. Fiber supercapacitors made of nanowire-fiber hybrid structures for wearable/flexible energy storage. Angew. Chem. Int. Ed. 2011, 50, 1683-1687.

[98] Na, J. S.; Gong, B.; Scarel, G.; Parsons, G. N. Surface polarity shielding and hierarchical $\mathrm{ZnO}$ nano-architectures produced using sequential hydrothermal crystal synthesis and thin film atomic layer deposition. ACS Nano 2009, 3, 3191-3199.

[99] Kang, B. S.; Pearton, S. J.; Ren, F. Low temperature (<100 degrees C) patterned growth of $\mathrm{ZnO}$ nanorod arrays on Si. Appl. Phys. Lett. 2007, 90, 083104. 
[100] Xu, S.; Wei, Y. G.; Liu, J.; Yang, R.; Wang, Z. L. Integrated multilayer nanogenerator fabricated using paired nanotip-to-nanowire brushes. Nano Lett. 2008, 8, 4027-4032.

[101] Sun, H. K.; Luo, M.; Weng, W. J.; Cheng, K.; Du, P.; Shen, G.; Han, G. R. Position and density control in hydrothermal growth of $\mathrm{ZnO}$ nanorod arrays through pre-formed micro/ nanodots. Nanotechnology 2008, 19, 395602.

[102] Ma, T.; Guo, M.; Zhang, M.; Zhang, Y. J.; Wang, X. D. Density-controlled hydrothermal growth of well-aligned ZnO nanorod arrays. Nanotechnology 2007, 18, 035605.

[103] Hsiao, C. S.; Peng, C. H.; Chen, S. Y.; Liou, S. C. Tunable growth of $\mathrm{ZnO}$ nanorods synthesized in aqueous solutions at low temperatures. J. Vac. Sci. Technol. B 2006, 24, 288-291.

[104] Qiu, J. J.; Li, X. M.; He, W. Z.; Park, S. J.; Kim, H. K.; Hwang, Y. H.; Lee, J. H.; Kim, Y. D. The growth mechanism and optical properties of ultralong $\mathrm{ZnO}$ nanorod arrays with a high aspect ratio by a preheating hydrothermal method. Nanotechnology 2009, 20, 155603.

[105] Cao, X. L.; Zeng, H. B.; Wang, M.; Xu, X. J.; Fang, M.; Ji, S. L.; Zhang, L. D. Large scale fabrication of quasi-aligned ZnO stacking nanoplates. J. Phys. Chem. C 2008, 112, 52675270.

[106] Greene, L. E.; Law, M.; Tan, D. H.; Montano, M.; Goldberger, J.; Somorjai, G.; Yang, P. D. General route to vertical $\mathrm{ZnO}$ nanowire arrays using textured $\mathrm{ZnO}$ seeds. Nano Lett. 2005, 5, 1231-1236.

[107] Yang, Y.; Chu, Y.; Zhang, Y. P.; Yang, F. Y.; Liu, J. L. Polystyrene-ZnO core-shell microspheres and hollow $\mathrm{ZnO}$ structures synthesized with the sulfonated polystyrene templates. J. Solid State Chem. 2006, 179, 470-475.

[108] Fang, Y. P.; Pang, Q.; Wen, X. G.; Wang, B. N.; Yang, S. $\mathrm{H}$. Synthesis of ultrathin $\mathrm{ZnO}$ nanofibers aligned on a zinc substrate. Small 2006, 2, 612-615.

[109] Kar, S.; Dev, A.; Chaudhuri, S. Simple solvothermal route to synthesize $\mathrm{ZnO}$ nanosheets, nanonails, and well-aligned nanorod arrays. J. Phys. Chem. B 2006, 110, 17848-17853.

[110] Peterson, R. B.; Fields, C. L.; Gregg, B. A. Epitaxial chemical deposition of $\mathrm{ZnO}$ nanocolumns from $\mathrm{NaOH}$ solutions. Langmuir 2004, 20, 5114-5118.

[111] Zhou, Z. Z.; Deng, Y. L. Kinetics study of ZnO nanorod growth in solution. J. Phys. Chem. C 2009, 113, 1985319858.

[112] Liu, J.; She, J. C.; Deng, S. Z.; Chen, J.; Xu, N. S. Ultrathin seed-layer for tuning density of $\mathrm{ZnO}$ nanowire arrays and their field emission characteristics. J. Phys. Chem. C 2008, 112, 11685-11690.

[113] Weintraub, B.; Chang, S.; Singamaneni, S.; Han, W. H.; Choi, Y. J.; Bae, J. H.; Kirkham, M.; Tsukruk, V. V.; Deng, Y. L. Density-controlled, solution-based growth of $\mathrm{ZnO}$ nanorod arrays via layer-by-layer polymer thin films for enhanced field emission. Nanotechnology 2008, 19, 435302.

[114] Gao, P. X.; Song, J. H.; Liu, J.; Wang, Z. L. Nanowire piezoelectric nanogenerators on plastic substrates as flexible power sources for nanodevices. Adv. Mater. 2007, 19, 67-72.

[115] Zeng, H. B.; Cui, J. B.; Cao, B. Q.; Gibson, U.; Bando, Y.; Golberg, D. Electrochemical deposition of $\mathrm{ZnO}$ nanowire arrays: Organization, doping, and properties. Sci. Adv. Mater. 2010, 2, 336-358.

[116] Izaki, M.; Watanabe, M.; Aritomo, H.; Yamaguchi, I.; Asahina, S.; Shinagawa, T.; Chigane, M.; Inaba, M.; Tasaka, A. Zinc oxide nano-cauliflower array with room temperature ultraviolet light emission. Cryst. Growth Des. 2008, 8, 1418-1421.

[117] Yu, L. G.; Zhang, G. M.; Li, S. Q.; Xi, Z. H.; Guo, D. Z. Fabrication of arrays of zinc oxide nanorods and nanotubes in aqueous solution under an external voltage. J. Cryst. Growth 2007, 299, 184-188.

[118] Konenkamp, R.; Boedecker, K.; Lux-Steiner, M. C.; Poschenrieder, M.; Zenia, F.; Levy-Clement, C.; Wagner, S. Thin film semiconductor deposition on free-standing $\mathrm{ZnO}$ columns. Appl. Phys. Lett. 2000, 77, 2575-2577.

[119] Cui, J. B.; Soo, Y. C.; Chen, T. P.; Gibson, U. J. Lowtemperature growth and characterization of Cl-doped $\mathrm{ZnO}$ nanowire arrays. J. Phys. Chem. C 2008, 112, 4475-4479.

[120] Cui, J. B.; Gibson, U. J. Electrodeposition and room temperature ferromagnetic anisotropy of $\mathrm{Co}$ and $\mathrm{Ni}$-doped ZnO nanowire arrays. Appl. Phys. Lett. 2005, 87, 133108.

[121] Elias, J.; Tena-Zaera, R.; Levy-Clement, C. Electrochemical deposition of $\mathrm{ZnO}$ nanowire arrays with tailored dimensions. J. Electroanal. Chem. 2008, 621, 171-177.

[122] Zhao, J.; Jin, Z. G.; Li, T.; Liu, X. X.; Liu, Z. F. Growth of $\mathrm{ZnO}$ nanorods by the chemical solution method with assisted electrical field. J. Am. Ceram. Soc. 2006, 89, 2654-2659.

[123] Elias, J.; Tena-Zaera, R.; Levy-Clement, C. Effect of the chemical nature of the anions on the electrodeposition of $\mathrm{ZnO}$ nanowire arrays. J. Phys. Chem. C 2008, 112, 5736-5741.

[124] Xu, L. F.; Guo, Y.; Liao, Q.; Zhang, J. P.; Xu, D. S. Morphological control of $\mathrm{ZnO}$ nanostructures by electrodeposition. J. Phys. Chem. B 2005, 109, 13519-13522.

[125] Tena-Zaera, R.; Elias, J.; Wang, G.; Levy-Clement, C. Role of chloride ions on electrochemical deposition of $\mathrm{ZnO}$ nanowire arrays from O-2 reduction. J. Phys. Chem. C 2007, 111, 16706-16711.

[126] Tena-Zaera, R.; Elias, J.; Levy-Clement, C.; Bekeny, C.; Voss, T.; Mora-Sero, I.; Bisquert, J. Influence of the potassium chloride concentration on the physical properties of electrodeposited $\mathrm{ZnO}$ nanowire arrays. J. Phys. Chem. C 2008, 112, 16318-16323. 
[127] Anthony, S. P.; Lee, J. I.; Kim, J. K. Tuning optical band gap of vertically aligned $\mathrm{ZnO}$ nanowire arrays grown by homoepitaxial electrodeposition. Appl. Phys. Lett. 2007, 90, 103107.

[128] Zheng, M. J.; Zhang, L. D.; Li, G. H.; Shen, W. Z. Fabrication and optical properties of large-scale uniform zinc oxide nanowire arrays by one-step electrochemical deposition technique. Chem. Phys. Lett. 2002, 363, 123-128.

[129] Zhou, H. J.; Wong, S. S. A facile and mild synthesis of 1-D $\mathrm{ZnO}, \mathrm{CuO}$, and alpha- $\mathrm{Fe}_{2} \mathrm{O}_{3}$ nanostructures and nanostructured arrays. ACS Nano 2008, 2, 944-958.

[130] Liu, B.; Zeng, H. C. Fabrication of ZnO "dandelions" via a modified Kirkendall process. J. Am. Chem. Soc. 2004, 126 16744-16746.

[131] Cong, H. P.; Yu, S. H. Hybrid ZnO-dye hollow spheres with new optical properties from a self-assembly process based on Evans blue dye and cetyltrimethylammonium bromide. Adv. Funct. Mater. 2007, 17, 1814-1820.

[132] Zhang, J.; Sun, L. D.; Pan, H. Y.; Liao, C. S.; Yan, C. H. $\mathrm{ZnO}$ nanowires fabricated by a convenient route. New J. Chem. 2002, 26, 33-34.

[133] Sun, X. M.; Chen, X.; Deng, Z. X.; Li, Y. D. A CTABassisted hydrothermal orientation growth of $\mathrm{ZnO}$ nanorods. Mater. Chem. Phys. 2003, 78, 99-104.

[134] Zhang, H.; Yang, D.; Ji, Y. J.; Ma, X. Y; Xu, J.; Que, D. L. Low temperature synthesis of flowerlike $\mathrm{ZnO}$ nanostructures by cetyltrimethylammonium bromide-assisted hydrothermal process. J. Phys. Chem. B 2004, 108, 3955-3958.

[135] Atanasova, P.; Weitz, R. T.; Gerstel, P.; Srot, V.; Kopold, P.; van Aken, P. A.; Burghard, M.; Bill, J. DNA-templated synthesis of $\mathrm{ZnO}$ thin layers and nanowires. Nanotechnology 2009, 20, 365302.

[136] Kim, J. H.; Kim, E. M.; Andeen, D.; Thomson, D.; DenBaars, S. P.; Lange, F. F. Growth of heteroepitaxial $\mathrm{ZnO}$ thin films on $\mathrm{GaN}$-buffered $\mathrm{Al}_{2} \mathrm{O}_{3}(0001)$ substrates by lowtemperature hydrothermal synthesis at $90{ }^{\circ} \mathrm{C}$. Adv. Funct. Mater. 2007, 17, 463-471.

[137] Shen, L. M.; Bao, N. Z.; Yanagisawa, K.; Zheng, Y. Q.; Domen, K.; Gupta, A.; Grimes, C. A. Direct growth of comet-like superstructures of $\mathrm{Au}-\mathrm{ZnO}$ submicron rod arrays by solvothermal soft chemistry process. J. Solid State Chem. 2007, 180, 213-220.

[138] Gao, P. X.; Lee, J. L.; Wang, Z. L. Multicolored ZnO nanowire architectures on trenched silicon substrates. $J$. Phys. Chem. C 2007, 111, 13763-13769.

[139] Lee, J. Y.; Yin, D. H.; Horiuchi, S. Site and morphology controlled $\mathrm{ZnO}$ deposition on Pd catalyst prepared from Pd/PMMA thin film using UV lithography. Chem. Mater. 2005, 17, 5498-5503.
[140] Xu, C. X.; Wei, A.; Sun, X. W.; Dong, Z. L. Aligned ZnO nanorods synthesized by a simple hydrothermal reaction. $J$. Phys. D: Appl. Phys. 2006, 39, 1690-1693.

[141] Cao, B. Q.; Cai, W. P.; Duan, G. T.; Li, Y.; Zhao, Q.; Yu, D. P. A template-free electrochemical deposition route to $\mathrm{ZnO}$ nanoneedle arrays and their optical and field emission properties. Nanotechnology 2005, 16, 2567-2574.

[142] Liu, R.; Vertegel, A. A.; Bohannan, E. W.; Sorenson, T. A.; Switzer, J. A. Epitaxial electrodeposition of zinc oxide nanopillars on single-crystal gold. Chem. Mater. 2001, 13, 508-512.

[143] Niarchos, G.; Makarona, E.; Tsamis, C. Growth of ZnO nanorods on patterned templates for efficient, large-area energy scavengers. Microsyst. Technol. 2010, 16, 669-675.

[144] Ahsanulhaq, Q.; Umar, A.; Hahn, Y. B. Growth of aligned $\mathrm{ZnO}$ nanorods and nanopencils on $\mathrm{ZnO} / \mathrm{Si}$ in aqueous solution: Growth mechanism and structural and optical properties. Nanotechnology 2007, 18, 115603.

[145] Nayak, J.; Sahu, S. N.; Kasuya, J.; Nozaki, S. Effect of substrate on the structure and optical properties of $\mathrm{ZnO}$ nanorods. J. Phys. D: Appl. Phys. 2008, 41, 115303.

[146] Zhou, H. L.; Chen, A.; Jian, L. K.; Ooi, K. F.; Goh, G. K. L.; Zang, K. Y.; Chua, S. J. Template-directed selective growth of ordered $\mathrm{ZnO}$ nanostructures on $\mathrm{GaN}$ by the hydrothermal method. J. Cryst. Growth 2008, 310, 3626-3629.

[147] Cole, J. J.; Wang, X.; Knuesel, R. J.; Jacobs, H. O. Integration of $\mathrm{ZnO}$ microcrystals with tailored dimensions forming light emitting diodes and UV photovoltaic cells. Nano Lett. 2008, 8, 1477-1481.

[148] Lee, S. D.; Kim, Y. S.; Yi, M. S.; Choi, J. Y.; Kim, S. W. Morphology control and electroluminescence of $\mathrm{ZnO}$ nanorod/GaN heterojunctions prepared using aqueous solution. J. Phys. Chem. C 2009, 113, 8954-8958.

[149] Cole, J. J.; Wang, X. Y.; Knuesel, R. J.; Jacobs, H. O. Patterned growth and transfer of $\mathrm{ZnO}$ micro- and nanocrystals with size and location control. Adv. Mater. 2008, 20, 14741478.

[150] Kim, J. H.; Andeen, D.; Lange, F. F. Hydrothermal growth of periodic, single-crystal $\mathrm{ZnO}$ microrods and microtunnels. Adv. Mater. 2006, 18, 2453-2457.

[151] Le, H. Q.; Chua, S. J.; Koh, Y. W.; Loh, K. P.; Chen, Z.; Thompson, C. V.; Fitzgerald, E. A. Growth of single crystal $\mathrm{ZnO}$ nanorods on $\mathrm{GaN}$ using an aqueous solution method. Appl. Phys. Lett. 2005, 87, 101908.

[152] Pauporte, T.; Lincot, D.; Viana, B.; Pelle, F. Toward laser emission of epitaxial nanorod arrays of $\mathrm{ZnO}$ grown by electrodeposition. Appl. Phys. Lett. 2006, 89, 233112.

[153] Le, H. Q.; Chua, S. J.; Loh, K. P.; Fitzgerald, E. A.; Koh, Y. W. Synthesis and optical properties of well aligned $\mathrm{ZnO}$ 
nanorods on $\mathrm{GaN}$ by hydrothermal synthesis. Nanotechnology 2006, 17, 483-488.

[154] Zhang, H.; Yang, D. R.; Li, D. S.; Ma, X. Y.; Li, S. Z.; Que, D. L. Controllable growth of $\mathrm{ZnO}$ microcrystals by a capping-molecule-assisted hydrothermal process. Cryst. Growth Des. 2005, 5, 547-550.

[155] Wu, W. B.; Hu, G. D.; Cui, S. G.; Zhou, Y.; Wu, H. T. Epitaxy of vertical $\mathrm{ZnO}$ nanorod arrays on highly (001)oriented $\mathrm{ZnO}$ seed monolayer by a hydrothermal route. Cryst. Growth Des. 2008, 8, 4014-4020.

[156] Zhou, Y.; Wu, W. B.; Hu, G. D.; Wu, H. T.; Cui, S. G. Hydrothermal synthesis of $\mathrm{ZnO}$ nanorod arrays with the addition of polyethyleneimine. Mater. Res. Bull. 2008, 43, 2113-2118.

[157] Tian, Z. R. R.; Voigt, J. A.; Liu, J.; McKenzie, B.; McDermott, M. J. Biomimetic arrays of oriented helical $\mathrm{ZnO}$ nanorods and columns. J. Am. Chem. Soc. 2002, 124, 12954-12955.

[158] Yang, Z.; Liu, Q. H.; Yu, H. C.; Zou, B. S.; Wang, Y. G.; Wang, T. H. Substrate-free growth, characterization and growth mechanism of $\mathrm{ZnO}$ nanorod close-packed arrays. Nanotechnology 2008, 19, 035704.

[159] Mclaren, A.; Valdes-Solis, T.; Li, G. Q.; Tsang, S. C. Shape and size effects of $\mathrm{ZnO}$ nanocrystals on photocatalytic activity. J. Am. Chem. Soc. 2009, 131, 12540-12541.

[160] Hidber, P. C.; Graule, T. J.; Gauckler, L. J. Citric acid-A dispersant for aqueous alumina suspensions. J. Am. Ceram. Soc. 1996, 79, 1857-1867.

[161] Tian, Z. R. R.; Voigt, J. A.; Liu, J.; McKenzie, B.; McDermott, M. J.; Rodriguez, M. A.; Konishi, H.; Xu, H. F. Complex and oriented $\mathrm{ZnO}$ nanostructures. Nat. Mater. 2003, 2, 821-826.

[162] Liang, L.; Liu, J.; Windisch, C. F.; Exarhos, G. J.; Lin, Y. H. Direct assembly of large arrays of oriented conducting polymer nanowires. Angew. Chem. Int. Ed. 2002, 41, 36653668.

[163] Tian, Z. R. R.; Voigt, J. A.; Liu, J.; McKenzie, B.; Xu, H. F. Large oriented arrays and continuous films of $\mathrm{TiO}_{2}$-based nanotubes. J. Am. Chem. Soc. 2003, 125, 12384-12385.

[164] Yan, J.; Fang, X. S.; Zhang, L. D.; Bando, Y.; Gautam, U. K.; Dierre, B.; Sekiguchi, T.; Golberg, D. Structure and cathodoluminescence of individual $\mathrm{ZnS} / \mathrm{ZnO}$ biaxial nanobelt heterostructures. Nano Lett. 2008, 8, 2794-2799.

[165] Choopun, S.; Hongsith, N.; Tanunchai, S.; Chairuangsri, T.; Krua-in, C.; Singkarat, S.; Vilaithonga, T.; Mangkorntong, P.; Mangkorntong, N. Single-crystalline $\mathrm{ZnO}$ nanobelts by RF sputtering. J. Cryst. Growth 2005, 282, 365-369.

[166] Wei, Y. G.; Ding, Y.; Li, C.; Xu, S.; Ryo, J. H.; Dupuis, R.; Sood, A. K.; Polla, D. L.; Wang, Z. L. Growth of vertically aligned $\mathrm{ZnO}$ nanobelt arrays on GaN substrate. J. Phys. Chem. C 2008, 112, 18935-18937.

[167] Xi, Y.; Hu, C. G.; Han, X. Y.; Xiong, Y. F.; Gao, P. X.; Liu, G. B. Hydrothermal synthesis of $\mathrm{ZnO}$ nanobelts and gas sensitivity property. Solid State Commun. 2007, 141, 506-509.

[168] Yang, J. H.; Liu, G. M.; Lu, J.; Qiu, Y. F.; Yang, S. H. Electrochemical route to the synthesis of ultrathin $\mathrm{ZnO}$ nanorod/nanobelt arrays on zinc substrate. Appl. Phys. Lett. 2007, 90, 103109

[169] Song, R. Q.; Xu, A. W.; Deng, B.; Li, Q.; Chen, G. Y. From layered basic zinc acetate nanobelts to hierarchical zinc oxide nanostructures and porous zinc oxide nanobelts. $A d v$. Funct. Mater. 2007, 17, 296-306.

[170] Vayssieres, L.; Keis, K.; Hagfeldt, A.; Lindquist, S. E. Three-dimensional array of highly oriented crystalline $\mathrm{ZnO}$ microtubes. Chem. Mater. 2001, 13, 4395-4398.

[171] Zhang, J.; Sun, L. D.; Liao, C. S.; Yan, C. H. A simple route towards tubular $\mathrm{ZnO}$. Chem. Commun. 2002, 262-263.

[172] She, G. W.; Zhang, X. H.; Shi, W. S.; Fan, X.; Chang, J. C. Electrochemical/chemical synthesis of highly-oriented singlecrystal $\mathrm{ZnO}$ nanotube arrays on transparent conductive substrates. Electrochem. Commun. 2007, 9, 2784-2788.

[173] Sun, Y.; Fuge, G. M.; Fox, N. A.; Riley, D. J.; Ashfold, M. N. R. Synthesis of aligned arrays of ultrathin $\mathrm{ZnO}$ nanotubes on a Si wafer coated with a thin $\mathrm{ZnO}$ film. Adv. Mater. 2005 , 17, 2477-2481.

[174] Jiang, H.; Hu, J.; Gu, F.; Li, C. Self-assembly of solid or tubular $\mathrm{ZnO}$ rods into twinning microprisms via a hydrothermal route. J. Alloys Compd. 2009, 478, 550-553.

[175] Wang, Z.; Qian, X. F.; Yin, J.; Zhu, Z. K. Large-scale fabrication of tower-like, flower-like, and tube-like $\mathrm{ZnO}$ arrays by a simple chemical solution route. Langmuir 2004, 20, 3441-3448.

[176] Yu, K.; Jin, Z. G.; Liu, X. X.; Zhao, J.; Feng, J. Y. Shape alterations of $\mathrm{ZnO}$ nanocrystal arrays fabricated from $\mathrm{NH}_{3} \cdot \mathrm{H}_{2} \mathrm{O}$ solutions. Appl. Surf. Sci. 2007, 253, 4072-4078.

[177] Xi, Y.; Song, J. H.; Xu, S.; Yang, R. S.; Gao, Z. Y.; Hu, C. G.; Wang, Z. L. Growth of $\mathrm{ZnO}$ nanotube arrays and nanotube based piezoelectric nanogenerators. J. Mater. Chem. 2009, 19, 9260-9264.

[178] Yang, A. L.; Cui, Z. L. ZnO layer and tubular structures synthesized by a simple chemical solution route. Mater. Lett. 2006, 60, 2403-2405.

[179] Elias, J.; Tena-Zaera, R.; Wang, G. Y.; Levy-Clement, C. Conversion of $\mathrm{ZnO}$ nanowires into nanotubes with tailored dimensions. Chem. Mater. 2008, 20, 6633-6637.

[180] Yu, H. D.; Zhang, Z. P.; Han, M. Y.; Hao, X. T.; Zhu, F. R. A general low-temperature route for large-scale fabrication 
of highly oriented $\mathrm{ZnO}$ nanorod/nanotube arrays. $J$. Am. Chem. Soc. 2005, 127, 2378-2379.

[181] Israr, M. Q.; Sadaf, J. R.; Yang, L. L.; Nur, O.; Willander, M.; Palisaitis, J.; Persson, P. O. A. Trimming of aqueous chemically grown $\mathrm{ZnO}$ nanorods into $\mathrm{ZnO}$ nanotubes and their comparative optical properties. Appl. Phys. Lett. 2009, 95, 073114.

[182] Sun, Y.; Riley, D. J.; Ashfold, M. N. R. Mechanism of $\mathrm{ZnO}$ nanotube growth by hydrothermal methods on $\mathrm{ZnO}$ film-coated Si substrates. J. Phys. Chem. B 2006, 110, 15186-15192.

[183] Li, Q. C.; Kumar, V.; Li, Y.; Zhang, H. T.; Marks, T. J.; Chang, R. P. H. Fabrication of $\mathrm{ZnO}$ nanorods and nanotubes in aqueous solutions. Chem. Mater. 2005, 17, 1001-1006.

[184] Tong, Y. H.; Liu, Y. C.; Shao, C. L.; Liu, Y. X.; Xu, C. S.; Zhang, J. Y.; Lu, Y. M.; Shen, D. Z.; Fan, X. W. Growth and optical properties of faceted hexagonal $\mathrm{ZnO}$ nanotubes. J. Phys. Chem. B 2006, 110, 14714-14718.

[185] Tong, Y. H.; Liu, Y. C.; Dong, L.; Zhao, D. X.; Zhang, J. Y.; Lu, Y. M.; Shen, D. Z.; Fan, X. W. Growth of ZnO nanostructures with different morphologies by using hydrothermal technique. J. Phys. Chem. B 2006, 110, 20263-20267.

[186] She, G. W.; Zhang, X. H.; Shi, W. S.; Fan, X.; Chang, J. C.; Lee, C. S.; Lee, S. T.; Liu, C. H. Controlled synthesis of oriented single-crystal $\mathrm{ZnO}$ nanotube arrays on transparent conductive substrates. Appl. Phys. Lett. 2008, 92, 053111.

[187] Li, F.; Ding, Y.; Gao, P. X. X.; Xin, X. Q.; Wang, Z. L. Single-cystal hexagonal disks and rings of $\mathrm{ZnO}$ : Lowtemperature, large-scale synthesis and growth mechanism. Angew. Chem. Int. Ed. 2004, 43, 5238-5242.

[188] Magalhaes, M.; Pusiol, D.; Ramia, M. E.; Neto, A. M. F. Phase diagram of a lyotropic mixture sodium bis (2-ethylhexyl) sulfosuccinate/dodecanol/water: Reverse micellar, cylindrical, lamellar, and sponge phases. J. Chem. Phys. 1998, 108, 3835-3843.

[189] Jung, S.; Cho, W.; Lee, H. J.; Oh, M. Self-template-directed formation of coordination-polymer hexagonal tubes and rings, and their calcination to $\mathrm{ZnO}$ rings. Angew. Chem. Int. Ed. 2009, 48, 1459-1462.

[190] Liu, X. G. Zinc oxide nano- and microfabrication from coordination-polymer templates. Angew. Chem. Int. Ed. 2009, 48, 3018-3021.

[191] Oner, M.; Norwig, J.; Meyer, W. H.; Wegner, G. Control of $\mathrm{ZnO}$ crystallization by a PEO-b-PMAA diblock copolymer. Chem. Mater. 1998, 10, 460-463.

[192] Taubert, A.; Kubel, C.; Martin, D. C. Polymer-induced microstructure variation in zinc oxide crystals precipitated from aqueous solution. J. Phys. Chem. B 2003, 107, 26602666.
[193] Zhang, S.; Shen, Y.; Fang, H.; Xu, S.; Song, J. H.; Wang, Z. L. Growth and replication of ordered $\mathrm{ZnO}$ nanowire arrays on general flexible substrates. J. Mater. Chem. 2010, 20, 10606-10610.

[194] Wang, B. G.; Shi, E. W.; Zhong, W. Z. Twinning morphologies and mechanisms of $\mathrm{ZnO}$ crystallites under hydrothermal conditions. Cryst. Res. Techol. 1998, 33, 937941.

[195] Zhang, T. R.; Dong, W. J.; Keeter-Brewer, M.; Konar, S.; Njabon, R. N.; Tian, Z. R. Site-specific nucleation and growth kinetics in hierarchical nanosyntheses of branched ZnO crystallites. J. Am. Chem. Soc. 2006, 128, 10960-10968.

[196] Zhang, T. R.; Dong, W. J.; Njabon, R. N.; Varadan, V. K.; Tian, Z. R. Kinetically probing site-specific heterogeneous nucleation and hierarchical growth of nanobranches. $J$. Phys. Chem. C 2007, 111, 13691-13695.

[197] Sounart, T. L.; Liu, J.; Voigt, J. A.; Hsu, J. W. P.; Spoerke, E. D.; Tian, Z.; Jiang, Y. B. Sequential nucleation and growth of complex nanostructured films. Adv. Funct. Mater. 2006, 16, 335-344.

[198] Sounart, T. L.; Liu, J.; Voigt, J. A.; Huo, M.; Spoerke, E. D.; McKenzie, B. Secondary nucleation and growth of $\mathrm{ZnO} . J$. Am. Chem. Soc. 2007, 129, 15786-15793.

[199] Ko, S. H.; Lee, D.; Kang, H. W.; Nam, K. H.; Yeo, J. Y.; Hong, S. J.; Grigoropoulos, C. P.; Sung, H. J. Nanoforest of hydrothermally grown hierarchical $\mathrm{ZnO}$ nanowires for a high efficiency dye-sensitized solar cell. Nano Lett. 2011, 11, 666-671.

[200] Mo, M.; Yu, J. C.; Zhang, L. Z.; Li, S. K. A. Selfassembly of $\mathrm{ZnO}$ nanorods and nanosheets into hollow microhemispheres and microspheres. Adv. Mater. 2005, 17, 756-760.

[201] Liu, B.; Zeng, H. C. Hollow ZnO microspheres with complex nanobuilding units. Chem. Mater. 2007, 19, 5824-5826.

[202] Penn, R. L.; Banfield, J. F. Imperfect oriented attachment: Dislocation generation in defect-free nanocrystals. Science 1998, 281, 969-971.

[203] Koh, Y. W.; Loh, K. P. Hexagonally packed zinc oxide nanorod bundles on hydrotalcite sheets. J. Mater. Chem. 2005, 15, 2508-2514.

[204] Chow, L.; Lupan, O.; Heinrich, H.; Chai, G. Self-assembly of densely packed and aligned bilayer $\mathrm{ZnO}$ nanorod arrays. Appl. Phys. Lett. 2009, 94, 163105.

[205] Thevenot, F.; Szymanski, R.; Chaumette, P. Preparation and characterization of Al-rich $\mathrm{Zn}-\mathrm{Al}$ hydrotalcite-like compounds. Clays Clay Miner. 1989, 37, 396-402.

[206] Liu, J. P.; Huang, X. T.; Li, Y. Y.; Sulieman, K. M.; He, X.; Sun, F. L. Facile and large-scale production of $\mathrm{ZnO} / \mathrm{Zn}-\mathrm{Al}$ layered double hydroxide hierarchical heterostructures. $J$. 
Phys. Chem. B 2006, 110, 21865-21872.

[207] Zhang, H.; Yang, D. R.; Ma, X. Y.; Que, D. L. Synthesis and field emission characteristics of bilayered $\mathrm{ZnO}$ nanorod array prepared by chemical reaction. J. Phys. Chem. B 2005, 109, 17055-17059.

[208] Li, H. X.; Xia, M. X.; Dai, G. Z.; Yu, H. C.; Zhang, Q. L.; Pan, A. L.; Wang, T. H.; Wang, Y. G.; Zou, B. S. Growth of oriented zinc oxide nanowire array into novel hierarchical structures in aqueous solutions. J. Phys. Chem. C 2008, 112, 17546-17553.

[209] Xu, C. K.; Wu, J. M.; Desai, U. V.; Gao, D. Multilayer assembly of nanowire arrays for dye-sensitized solar cells. J. Am. Chem. Soc. 2011, 133, 8122-8125.

[210] Liu, L.; Fu, L.; Liu, Y.; Liu, Y. L.; Jiang, P.; Liu, S. Q.; Gao, M. Y.; Tang, Z. Y. Bioinspired synthesis of vertically aligned $\mathrm{ZnO}$ nanorod arrays: Toward greener chemistry. Cryst. Growth Des. 2009, 9, 4793-4796.

[211] Tang, Y. W.; Hu, X. Y.; Chen, M. J.; Luo, L. J.; Li, B. H.; Zhang, L. Z. CdSe nanocrystal sensitized $\mathrm{ZnO}$ core-shell nanorod array films: Preparation and photovoltaic properties. Electrochim. Acta 2009, 54, 2742-2747.

[212] Hao, Y. Z.; Pei, J.; Wei, Y.; Cao, Y. H.; Jiao, S. H.; Zhu, F.; Li, J. J.; Xu, D. H. Efficient semiconductor-sensitized solar cells based on poly(3-hexylthiophene)@CdSe@ZnO core-shell nanorod arrays. J. Phys. Chem. C 2010, 114, $8622-8625$.

[213] Wang, X. N.; Zhu, H. J.; Xu, Y. M.; Wang, H.; Tao, Y.; Hark, S.; Xiao, X. D.; Li, Q. A. Aligned ZnO/CdTe core-shell nanocable arrays on indium tin oxide: Synthesis and photoelectrochemical properties. ACS Nano 2010, 4, 3302-3308.

[214] Sounart, T. L.; Liu, J.; Voigt, J. A.; Hsu, J. W. P.; Spoerke, E. D.; Tian, Z.; Jiang, Y. B. Sequential nucleation and growth of complex nanostructured films. Adv. Funct. Mater. 2006, 16, 335-344.

[215] Shi, L.; Xu, Y. M.; Hark, S. K.; Liu, Y.; Wang, S.; Peng, L. M.; Wong, K. W.; Li, Q. Optical and electrical performance of $\mathrm{SnO}_{2}$ capped $\mathrm{ZnO}$ nanowire arrays. Nano Lett. 2007, 7, 3559-3563.

[216] Plank, N. O. V.; Snaith, H. J.; Ducati, C.; Bendall, J. S.; Schmidt-Mende, L.; Welland, M. E. A simple low temperature synthesis route for $\mathrm{ZnO}-\mathrm{MgO}$ core-shell nanowires. Nanotechnology 2008, 19, 465603.

[217] Plank, N. O. V.; Howard, I.; Rao, A.; Wilson, M. W. B.; Ducati, C.; Mane, R. S.; Bendall, J. S.; Louca, R. R. M.; Greenham, N. C.; Miura, H.; Friend, R. H.; Snaith, H. J.; Welland, M. E. Efficient $\mathrm{ZnO}$ nanowire solid-state dyesensitized solar cells using organic dyes and core-shell nanostructures. J. Phys. Chem. C 2009, 113, 18515-18522.
[218] Tak, Y.; Yong, K. A novel heterostructure of $\mathrm{Co}_{3} \mathrm{O}_{4} / \mathrm{ZnO}$ nanowire array fabricated by photochemical coating method. J. Phys. Chem. C 2008, 112, 74-79.

[219] Wang, Z.; Qian, X. F.; Li, Y.; Yin, J.; Zhu, Z. K. Largescale synthesis of tube-like $\mathrm{ZnS}$ and cable-like $\mathrm{ZnS}-\mathrm{ZnO}$ arrays: Preparation through the sulfuration conversion from $\mathrm{ZnO}$ arrays via a simple chemical solution route. J. Solid State Chem. 2005, 178, 1589-1594.

[220] Panda, S. K.; Dev, A.; Chaudhuri, S. Fabrication and luminescent properties of c-axis oriented $\mathrm{ZnO}-\mathrm{ZnS}$ core-shell and $\mathrm{ZnS}$ nanorod arrays by sulfidation of aligned $\mathrm{ZnO}$ nanorod arrays. J. Phys. Chem. C 2007, 111, 5039-5043.

[221] Chen, C. Y.; Lin, C. A.; Chen, M. J.; Lin, G. R.; He, J. H. $\mathrm{ZnO} / \mathrm{Al}_{2} \mathrm{O}_{3}$ core-shell nanorod arrays: Growth, structural characterization, and luminescent properties. Nanotechnology 2009, 20, 185605.

[222] Law, M.; Greene, L. E.; Radenovic, A.; Kuykendall, T.; Liphardt, J.; Yang, P. D. $\mathrm{ZnO}-\mathrm{Al}_{2} \mathrm{O}_{3}$ and $\mathrm{ZnO}-\mathrm{TiO}_{2}$ coreshell nanowire dye-sensitized solar cells. J. Phys. Chem. B 2006, 110, 22652-22663.

[223] Dergacheva, M. B.; Statsyuk, V. N.; Fogel, L. A. Electrodeposition of CdTe from ammonia-chloride buffer electrolytes. J. Electroanal. Chem. 2005, 579, 43-49.

[224] Kum, M. C.; Yoo, B. Y.; Rheem, Y.; Bozhilov, K. N.; Chen, W.; Mulchandani, A.; Myung, N. V. Synthesis and characterization of cadmium telluride nanowire. Nanotechnology 2008, 19, 325711.

[225] Xu, D. S.; Guo, Y. G.; Yu, D. P.; Guo, G. L.; Tang, Y. Q.; $\mathrm{Yu}, \mathrm{D}$. P. Highly ordered and well-oriented single-crystal CdTe nanowire arrays by direct-current electrodeposition. J. Mater. Res. 2002, 17, 1711-1714.

[226] Zhao, A. W.; Meng, G. W.; Zhang, L. D.; Gao, T.; Sun, S. H.; Pang, Y. T. Electrochemical synthesis of ordered CdTe nanowire arrays. Appl. Phys. A 2003, 76, 537-539.

[227] Cheng, C. W.; Liu, B.; Yang, H. Y.; Zhou, W. W.; Sun, L.; Chen, R.; Yu, S. F.; Zhang, J. X.; Gong, H.; Sun, H. D.; Fan, H. J. Hierarchical assembly of $\mathrm{ZnO}$ nanostructures on $\mathrm{SnO}_{2}$ backbone nanowires: Low-temperature hydrothermal preparation and optical properties. ACS Nano 2009, 3, 3069-3076.

[228] Dloczik, L.; Engelhardt, R.; Ernst, K.; Lux-Steiner, M. C.; Konenkamp, R. Zinc sulfide columns by chemical conversion of zinc oxide. Sens. Actuat. B-Chem. 2002, 84, 33-36.

[229] Dloczik, L.; Engelhardt, R.; Ernst, K.; Fiechter, S.; Sieber, I.; Konenkamp, R. Hexagonal nanotubes of $\mathrm{ZnS}$ by chemical conversion of monocrystalline $\mathrm{ZnO}$ columns. Appl. Phys. Lett. 2001, 78, 3687-3689.

[230] Qiu, J. J.; Yu, W. D.; Gao, X. D.; Li, X. M. Sol-gel assisted $\mathrm{ZnO}$ nanorod array template to synthesize $\mathrm{TiO}_{2}$ nanotube 
arrays. Nanotechnology 2006, 17, 4695-4698.

[231] Qui, J. J.; Jin, Z. G.; Liu, Z. F.; Liu, X. X.; Liu, G. Q.; Wu, W. B.; Zhang, X.; Gao, X. D. Fabrication of $\mathrm{TiO}_{2}$ nanotube film by well-aligned $\mathrm{ZnO}$ nanorod array film and sol-gel process. Thin Solid Films 2007, 515, 2897-2902.

[232] Dawood, F.; Schaak, R. E. ZnO-templated synthesis of wurtzite-type $\mathrm{ZnS}$ and $\mathrm{ZnSe}$ nanoparticles. J. Am. Chem. Soc. 2009, 131, 424-425.

[233] Alivisatos, A. P. Semiconductor clusters, nanocrystals, and quantum dots. Science 1996, 271, 933-937.

[234] Banholzer, M. J.; Millstone, J. E.; Qin, L. D.; Mirkin, C. A Rationally designed nanostructures for surface-enhanced Raman spectroscopy. Chem. Soc. Rev. 2008, 37, 885-897.

[235] Sakano, T.; Tanaka, Y.; Nishimura, R.; Nedyalkov, N. N.; Atanasov, P. A.; Saiki, T.; Obara, M. Surface enhanced Raman scattering properties using Au-coated $\mathrm{ZnO}$ nanorods grown by two-step, off-axis pulsed laser deposition. J. Phys. D: Appl. Phys. 2008, 41, 235304.

[236] Wood, A.; Giersig, M.; Mulvaney, P. Fermi level equilibration in quantum dot-metal nanojunctions. J. Phys. Chem. B 2001, 105, 8810-8815.

[237] Fan, L. Y.; Yu, S. H. ZnO@Co hybrid nanotube arrays growth from electrochemical deposition: Structural, optical, photocatalytic and magnetic properties. Phys. Chem. Chem. Phys. 2009, 11, 3710-3717.

[238] Pacholski, C.; Kornowski, A.; Weller, H. Site-specific photodeposition of silver on $\mathrm{ZnO}$ nanorods. Angew. Chem. Int. Ed. 2004, 43, 4774-4777.

[239] Fan, F. R.; Ding, Y.; Liu, D. Y.; Tian, Z. Q.; Wang, Z. L. Facet-selective epitaxial growth of heterogeneous nanostructures of semiconductor and metal: $\mathrm{ZnO}$ nanorods on Ag nanocrystals. J. Am. Chem. Soc. 2009, 131, 12036-12037.

[240] Ko, H.; Singamaneni, S.; Tsukruk, V. V. Nanostructured surfaces and assemblies as SERS media. Small 2008, 4, 1576-1599.

[241] He, H.; Cai, W. P.; Lin, Y. X.; Chen, B. S. Surface decoration of $\mathrm{ZnO}$ nanorod arrays by electrophoresis in the $\mathrm{Au}$ colloidal solution prepared by laser ablation in water. Langmuir 2010, 26, 8925-8932.

[242] Yang, S. K.; Cai, W. P.; Liu, G. Q.; Zeng, H. B. From nanoparticles to nanoplates: Preferential oriented connection of Ag colloids during electrophoretic deposition. J. Phys. Chem. C 2009, 113, 7692-7696.

[243] Li, P.; Wei, Z.; Wu, T.; Peng, Q.; Li, Y. D. Au-ZnO hybrid nanopyramids and their photocatalytic properties. J. Am. Chem. Soc. 2011, 133, 5660-5663.

[244] Trejo, M.; Santiago, P.; Sobral, H.; Rendon, L.; Pal, U. Synthesis and growth mechanism of one-dimensional $\mathrm{Zn} / \mathrm{ZnO}$ core-shell nanostructures in low-temperature hydrothermal process. Cryst. Growth Des. 2009, 9, 3024-3030.

[245] Zhang, W. D. Growth of $\mathrm{ZnO}$ nanowires on modified wellaligned carbon nanotube arrays. Nanotechnology 2006, 17, 1036-1040.

[246] Li, X. L.; Li, C.; Zhang, Y.; Chu, D. P.; Milne, W. I.; Fan, H. J. Atomic layer deposition of $\mathrm{ZnO}$ on multi-walled carbon nanotubes and its use for synthesis of CNT- $\mathrm{ZnO}$ heterostructures. Nanoscale Res. Lett. 2010, 5, 1836-1840.

[247] Zhang, W. D.; Jiang, L. C.; Ye, J. S. Photoelectrochemical study on charge transfer properties of $\mathrm{ZnO}$ nanowires promoted by carbon nanotubes. J. Phys. Chem. C 2009, 113, 16247-16253.

[248] Sernelius, B. E.; Berggren, K. F.; Jin, Z. C.; Hamberg, I.; Granqvist, C. G. Band-gap tailoring of $\mathrm{ZnO}$ by means of heavy Al doping. Phys. Rev. B 1988, 37, 10244-10248.

[249] Nadarajah, A.; Word, R. C.; Meiss, J.; Konenkamp, R. Flexible inorganic nanowire light-emitting diode. Nano Lett. 2008, 8, 534-537.

[250] Fang, T. H.; Kang, S. H. Electromechanical characteristics of ZnO:Al nanorods. J. Nanosci. Nanotechnol. 2010, 10, 405-412.

[251] Look, D. C.; Claflin, B.; Alivov, Y. I.; Park, S. J. The future of $\mathrm{ZnO}$ light emitters. Phys. Status Solidi A 2004, 201, 2203-2212.

[252] Xiang, B.; Wang, P. W.; Zhang, X. Z.; Dayeh, S. A.; Aplin, D. P. R.; Soci, C.; Yu, D. P.; Wang, D. L. Rational synthesis of $p$-type zinc oxide nanowire arrays using simple chemical vapor deposition. Nano Lett. 2007, 7, 323-328.

[253] Yuan, G. D.; Zhang, W. J.; Jie, J. S.; Fan, X.; Zapien, J. A.; Leung, Y. H.; Luo, L. B.; Wang, P. F.; Lee, C. S.; Lee, S. T. p-type ZnO nanowire arrays. Nano Lett. 2008, 8, 2591-2597.

[254] Hsu, Y. F.; Xi, Y. Y.; Tam, K. H.; Djurisic, A. B.; Luo, J. M.; Ling, C. C.; Cheung, C. K.; Ng, A. M. C.; Chan, W. K.; Deng, X.; Beling, C. D.; Fung, S.; Cheah, K. W.; Fong, P. W. K.; Surya, C. C. Undoped $p$-type $\mathrm{ZnO}$ nanorods synthesized by a hydrothermal method. Adv. Funct. Mater. 2008, 18, 1020-1030.

[255] Lin, C. C.; Chen, H. P.; Chen, S. Y. Synthesis and optoelectronic properties of arrayed $p$-type $\mathrm{ZnO}$ nanorods grown on $\mathrm{ZnO}$ film/Si wafer in aqueous solutions. Chem. Phys. Lett. 2005, 404, 30-34.

[256] Sun, M. H.; Zhang, Q. F.; Wu, J. L. Electrical and electroluminescence properties of As-doped $p$-type $\mathrm{ZnO}$ nanorod arrays. J. Phys. D: Appl. Phys. 2007, 40, 3798-3802.

[257] Thomas, M. A.; Cui, J. B. Electrochemical growth and characterization of Ag-doped $\mathrm{ZnO}$ nanostructures. J. Vac. Sci. Technol. B 2009, 27, 1673-1677.

[258] Yuhas, B. D.; Zitoun, D. O.; Pauzauskie, P. J.; Yang, P. Transition-metal doped zinc oxide nanowires. Angew. Chem. 
Int. Ed. 2006, 45, 420-423.

[259] Cui, J.; Gibson, U. J. Enhanced nucleation, growth rate, and dopant incorporation in $\mathrm{ZnO}$ nanowires. J. Phys. Chem. B 2005, 109, 22074-22077.

[260] Cui, J. B.; Zeng, Q.; Gibson, U. J. Synthesis and magnetic properties of Co-doped $\mathrm{ZnO}$ nanowires. J. Appl. Phys. 2006, 99, 08M113.

[261] Yuhas, B. D.; Fakra, S.; Marcus, M. A.; Yang, P. D. Probing the local coordination environment for transition metal dopants in zinc oxide nanowires. Nano Lett. 2007, 7, 905-909.

[262] Liang, W. J.; Yuhas, B. D.; Yang, P. D. Magnetotransport in Co-doped ZnO nanowires. Nano Lett. 2009, 9, 892-896.

[263] Gayen, R. N.; Das, S. N.; Dalui, S.; Bhar, R.; Pal, A. K. Zinc magnesium oxide nanofibers on glass substrate by solution growth technique. J. Cryst. Growth 2008, 310, 4073-4080.

[264] Fang, T. H.; Kang, S. H. Preparation and characterization of Mg-doped $\mathrm{ZnO}$ nanorods. J. Alloys Compd. 2010, 492, 536-542.

[265] Shimpi, P.; Gao, P. X.; Goberman, D. G.; Ding, Y. Low temperature synthesis and characterization of $\mathrm{MgO} / \mathrm{ZnO}$ composite nanowire arrays. Nanotechnology 2009, 20, 125608.

[266] Spanos, J. Photolithography applied to silicon transistor technology. J. Electrochem. Soc. 1961, 108, C176-C176.

[267] Boercker, J. E.; Schmidt, J. B.; Aydil, E. S. Transport limited growth of zinc oxide nanowires. Cryst. Growth Des. 2009, 9, 2783-2789.

[268] Coltrin, M. E.; Hsu, J. W. P.; Scrymgeour, D. A.; Creighton, J. R.; Simmons, N. C.; Matzke, C. M. Chemical kinetics and mass transport effects in solution-based selective-area growth of $\mathrm{ZnO}$ nanorods. J. Cryst. Growth 2008, 310, 584-593.

[269] Yi, S. H.; Choi, S. K.; Jang, J. M.; Kim, J. A.; Jung, W. G. Patterned growth of a vertically aligned zinc oxide rod array on a gallium nitride epitaxial layer by using a hydrothermal process. J. Korean Phys. Soc. 2008, 53, 227-231.

[270] Masuda, Y.; Kinoshita, N.; Sato, F.; Koumoto, K. Siteselective deposition and morphology control of UV- and visible-light-emitting $\mathrm{ZnO}$ crystals. Cryst. Growth Des. 2006, 6, 75-78.

[271] Morin, S. A.; Amos, F. F.; Jin, S. Biomimetic assembly of zinc oxide nanorods onto flexible polymers. J. Am. Chem. Soc. 2007, 129, 13776-13777.

[272] McCarley, R. L.; Vaidya, B.; Wei, S. Y.; Smith, A. F.; Patel, A. B.; Feng, J.; Murphy, M. C.; Soper, S. A. Resistfree patterning of surface architectures in polymer-based microanalytical devices. J. Am. Chem. Soc. 2005, 127,
842-843.

[273] Yang, P.; Zou, S. L.; Yang, W. T. Positive and negative $\mathrm{ZnO}$ micropatterning on functionatized polymer surfaces. Small 2008, 4, 1527-1536.

[274] Vieu, C.; Carcenac, F.; Pepin, A.; Chen, Y.; Mejias, M.; Lebib, A.; Manin-Ferlazzo, L.; Couraud, L.; Launois, H. Electron beam lithography: Resolution limits and applications. Appl. Surf. Sci. 2000, 164, 111-117.

[275] Kim, Y.; Lee, C.; Hong, Y. J.; Yi, G.; Kim, S. S.; Cheong, H. Controlled selective growth of $\mathrm{ZnO}$ nanorod and microrod arrays on Si substrates by a wet chemical method. Appl. Phys. Lett. 2006, 89, 163128.

[276] Lin, C. C.; Chen, S. Y.; Cheng, S. Y. Nucleation and growth behavior of well-aligned $\mathrm{ZnO}$ nanorods on organic substrates in aqueous solutions. J. Cryst. Growth 2005, 283, 141-146.

[277] Volk, J.; Nagata, T.; Erdelyi, R.; Barsony, I.; Toth, A. L.; Lukacs, I. E.; Czigany, Z.; Tomimoto, H.; Shingaya, Y.; Chikyow, T. Highly uniform epitaxial $\mathrm{ZnO}$ nanorod arrays for nanopiezotronics. Nanoscale Res. Lett. 2009, 4, 699-704.

[278] Liang, Y.; Zhen, C.; Zou, D.; Xu, D. Preparation of freestanding nanowire arrays on conductive substrates. J. Am. Chem. Soc. 2004, 126, 16338-16339.

[279] Dev, A.; Chaudhuri, S. Uniform large-scale growth of micropatterned arrays of $\mathrm{ZnO}$ nanowires synthesized by a surfactant assisted approach. Nanotechnology 2007, 18, 175607.

[280] Xu, S.; Ding, Y.; Wei, Y. G.; Fang, H.; Shen, Y.; Sood, A. K.; Polla, D. L.; Wang, Z. L. Patterned growth of horizontal ZnO nanowire arrays. J. Am. Chem. Soc. 2009, 131, 6670-6671.

[281] Wang, Z. L.; Yang, R. S.; Zhou, J.; Qin, Y.; Xu, C.; Hu, Y. F.; Xu, S. Lateral nanowire/nanobelt based nanogenerators, piezotronics and piezo-phototronics. Mat. Sci. Eng. R 2010, 70, 320-329.

[282] Qin, Y.; Yang, R. S.; Wang, Z. L. Growth of horizonatal $\mathrm{ZnO}$ nanowire arrays on any substrate. J. Phys. Chem. C 2008, 112, 18734-18736.

[283] Park, Y. K.; Choi, H. S.; Kim, J. H.; Kim, J. H.; Hahn, Y. B. High performance field-effect transistors fabricated with laterally grown $\mathrm{ZnO}$ nanorods in solution. Nanotechnology 2011, 22, 185310.

[284] Babak, N.; Chris, A. M.; Stephan, J. S.; Mark, D. V. Horizontal growth and in situ assembly of oriented zinc oxide nanowires. Appl. Phys. Lett. 2004, 85, 3244-3246.

[285] Harnack, O.; Pacholski, C.; Weller, H.; Yasuda, A.; Wessels, J. M. Rectifying behavior of electrically aligned $\mathrm{ZnO}$ nanorods. Nano Lett. 2003, 3, 1097-1101.

[286] Andeen, D. K. J. H.; Lang, F. F.; Goh, G. K. L.; Tripathy, S. Lateral epitaxial overgrowth of $\mathrm{ZnO}$ in water at $90{ }^{\circ} \mathrm{C}$. 
Adv. Funct. Mater. 2006, 16, 799-804.

[287] Saifullah, M. S. M.; Subramanian, K. R. V.; Kang, D. J.; Anderson, D.; Huck, W. T. S.; Jones, G. A. C.; Welland, M. E. Sub-10 nm high-aspect-ratio patterning of $\mathrm{ZnO}$ using an electron beam. Adv. Mater. 2005, 17, 1757-1761.

[288] Claeyssens, F.; Klini, A.; Mourka, A.; Fotakis, C. Laser patterning of $\mathrm{Zn}$ for $\mathrm{ZnO}$ nanostructure growth: Comparison between laser induced forward transfer in air and in vacuum. Thin Solid Films 2007, 515, 8529-8533.

[289] Guo, X. D.; Li, R. X.; Hang, Y.; Xu, Z. Z.; Yu, B. K.; Ma, H. L.; Lu, B.; Sun, X. W. Femtosecond laser-induced periodic surface structure on ZnO. Mater. Lett. 2008, 62, 1769-1771.

[290] Solak, H. H.; David, C.; Gobrecht, J.; Golovkina, V.; Cerrina, F.; Kim, S. O.; Nealey, P. F. Sub-50 nm period patterns with EUV interference lithography. Microelectron. Eng. 2003, 67-68, 56-62.

[291] Campbell, M.; Sharp, D. N.; Harrison, M. T.; Denning, R. G.; Turberfield, A. J. Fabrication of photonic crystals for the visible spectrum by holographic lithography. Nature 2000, 404, 53-56.

[292] Kim, K. S.; Jeong, H.; Jeong, M. S.; Jung, G. Y. Polymertemplated hydrothermal growth of vertically aligned singlecrystal $\mathrm{ZnO}$ nanorods and morphological transformations using structural polarity. Adv. Funct. Mater. 2010, 20, 30553063.

[293] Wei, Y.; Wu, W.; Guo, R.; Yuan, D.; Das, S.; Wang, Z. L. Wafer-scale high-throughput ordered growth of vertically aligned $\mathrm{ZnO}$ nanowire arrays. Nano Lett. 2010, 10, 34143419.

[294] Yuan, D. J.; Guo, R.; Wei, Y. G.; Wu, W. Z.; Ding, Y.; Wang, Z. L.; Das, S. Heteroepitaxial patterned growth of vertically aligned and periodically distributed $\mathrm{ZnO}$ nanowires on GaN using laser interference ablation. Adv. Funct. Mater. 2010, 20, 3484-3489.

[295] Haynes, C. L.; Van Duyne, R. P. Nanosphere lithography: A versatile nanofabrication tool for studies of size-dependent nanoparticle optics. J. Phys. Chem. B 2001, 105, 5599-5611.

[296] Zeng, H. B.; Xu, X. J.; Bando, Y.; Gautam, U. K.; Zhai, T. Y.; Fang, X. S.; Liu, B. D.; Golberg, D. Template deformation-tailored $\mathrm{ZnO}$ nanorod/nanowire arrays: Full growth control and optimization of field-emission. $A d v$. Funct. Mater. 2009, 19, 3165-3172.

[297] Li, C.; Hong, G. S.; Wang, P. W.; Yu, D. P.; Qi, L. M. Wet chemical approaches to patterned arrays of well-aligned $\mathrm{ZnO}$ nanopillars assisted by monolayer colloidal crystals. Chem. Mater. 2009, 21, 891-897.

[298] Chou, S. Y.; Krauss, P. R.; Renstrom, P. J. Imprint of sub-25 nm vias and trenches in polymers. Appl. Phys. Lett.
1995, 67, 3114-3116.

[299] Wang, C. H.; Wong, A. S. W.; Ho, G. W. Facile solution route to vertically aligned, selective growth of $\mathrm{ZnO}$ nanostructure arrays. Langmuir 2007, 23, 11960-11963.

[300] Kwon, S. J.; Park, J. H.; Park, J. G. Patterned growth of $\mathrm{ZnO}$ nanorods by micromolding of sol-gel-derived seed layer. Appl. Phys. Lett. 2005, 87, 133112.

[301] Whitesides, G. M.; Ostuni, E.; Takayama, S.; Jiang, X. Y.; Ingber, D. E. Soft lithography in biology and biochemistry. Annu. Rev. Biomed. Eng. 2001, 3, 335-373.

[302] Hsu, J. W. P.; Tian, Z. R.; Simmons, N. C.; Matzke, C. M.; Voigt, J. A.; Liu, J. Directed spatial organization of zinc oxide nanorods. Nano Lett. 2005, 5, 83-86.

[303] Hsu, J. W. P.; Tian, Z. R.; Simmons, N. C.; Matzke, C. M.; Voigt, J. A.; Liu, J. Spatial organization of $\mathrm{ZnO}$ nanorods on surfaces via organic templating. Proc. SPIE 2005, 5592, $158-163$.

[304] Lee, J. H.; Hon, M. H.; Chung, Y. W.; Leu, I. C. Microcontact printing of organic self-assembled monolayers for patterned growth of well-aligned $\mathrm{ZnO}$ nanorod arrays and their field-emission properties. J. Am. Ceram. Soc. 2009, 92, 2192-2196.

[305] Sirringhaus, H.; Kawase, T.; Friend, R. H.; Shimoda, T.; Inbasekaran, M.; Wu, W.; Woo, E. P. High-resolution inkjet printing of all-polymer transistor circuits. Science $\mathbf{2 0 0 0}$, 290, 2123-2126.

[306] Kitsomboonloha, R.; Baruah, S.; Myint, M. T. Z.; Subramanian, V.; Dutta, J. Selective growth of zinc oxide nanorods on inkjet printed seed patterns. J. Cryst. Growth 2009, 311, 2352-2358.

[307] de Gans, B. J.; Duineveld, P. C.; Schubert, U. S. Inkjet printing of polymers: State of the art and future developments. Adv. Mater. 2004, 16, 203-213.

[308] Sekitani, T.; Noguchi, Y.; Zschieschang, U.; Klauk, H.; Someya, T. Organic transistors manufactured using inkjet technology with subfemtoliter accuracy. Proc. Natl. Acad. Sci. USA 2008, 105, 4976-4980.

[309] Rogers, J. A.; Someya, T.; Huang, Y. G. Materials and mechanics for stretchable electronics. Science 2010, 327, 1603-1607.

[310] Hariharan, C. Photocatalytic degradation of organic contaminants in water by $\mathrm{ZnO}$ nanoparticles: Revisited. Appl. Catal. A 2006, 304, 55-61.

[311] Yang, X.; Wolcottt, A.; Wang, G.; Sobo, A.; Fitzmorris, R. C.; Qian, F.; Zhang, J. Z.; Li, Y. Nitrogen-doped ZnO nanowire arrays for photoelectrochemical water splitting. Nano Lett. 2009, 9, 2331-2336.

[312] Jang, E. S.; Won, J. H.; Hwang, S. J.; Choy, J. H. Fine tuning of the face orientation of $\mathrm{ZnO}$ crystals to optimize 
their photocatalytic activity. Adv. Mater. 2006, 18, 33093312.

[313] Akhavan, O. Graphene nanomesh by $\mathrm{ZnO}$ nanorod photocatalysts. ACS Nano 2010, 4, 4174-4180.

[314] Lu, F.; Cai, W. P.; Zhang, Y. G. ZnO hierarchical micro/ nanoarchitectures: Solvothermal synthesis and structurally enhanced photocatalytic performance. Adv. Funct. Mater. 2008, 18, 1047-1056.

[315] Wu, Q.; Chen, X.; Zhang, P.; Han, Y.; Chen, X.; Yan, Y.; $\mathrm{Li}, \mathrm{S}$. Amino acid-assisted synthesis of $\mathrm{ZnO}$ hierarchical architectures and their novel photocatalytic activities. Cryst. Growth Des. 2008, 8, 3010-3018.

[316] Kurtz, M.; Strunk, J.; Hinrichsen, O.; Muhler, M.; Fink, K.; Meyer, B.; Woll, C. Active sites on oxide surfaces: $\mathrm{ZnO}-$ catalyzed synthesis of methanol from $\mathrm{CO}$ and $\mathrm{H}_{2}$. Angew. Chem. Int. Ed. 2005, 44, 2790-2794.

[317] Lin, Y. G.; Hsu, Y. K.; Chen, S. Y.; Lin, Y. K.; Chen, L. C.; Chen, K. H. Nanostructured zinc oxide nanorods with copper nanoparticles as a microreformation catalyst. Angew. Chem. Int. Ed. 2009, 48, 7586-7590.

[318] Li, C. C.; Lin, R. J.; Lin, H. P.; Lin, Y. K.; Lin, Y. G.; Chang, C. C.; Chen, L. C.; Chen, K. H. Catalytic performance of plate-type $\mathrm{Cu} / \mathrm{Fe}$ nanocomposites on $\mathrm{ZnO}$ nanorods for oxidative steam reforming of methanol. Chem. Commun. 2011, 47, 1473-1475.

[319] Lin, Y. G.; Hsu, Y. K.; Chen, S. Y.; Chen, L. C.; Chen, K. $\mathrm{H}$. Microwave-activated $\mathrm{CuO}$ nanotip/ZnO nanorod nanoarchitectures for efficient hydrogen production. J. Mater. Chem. 2011, 21, 324-326.

[320] Boucher, M. B.; Yi, N.; Gittleson, F.; Zugic, B.; Saltsburg, H.; Flytzani-Stephanopoulos, M. Hydrogen production from methanol over gold supported on $\mathrm{ZnO}$ and $\mathrm{CeO}_{2}$ nanoshapes. J. Phys. Chem. C 2011, 115, 1261-1268.

[321] Tak, Y.; Kim, H.; Lee, D.; Yong, K. Type-II CdS nanoparticle- $\mathrm{ZnO}$ nanowire heterostructure arrays fabricated by a solution process: Enhanced photocatalytic activity. Chem. Commun. 2008, 4585-4587.

[322] Wang, W. W.; Zhu, Y. J.; Yang, L. X. ZnO--SnO 2 hollow spheres and hierarchical nanosheets: Hydrothermal preparation, formation mechanism, and photocatalytic properties. Adv. Funct. Mater. 2007, 17, 59-64.

[323] Tian, N.; Zhou, Z. Y.; Sun, S. G.; Ding, Y.; Wang, Z. L. Synthesis of tetrahexahedral platinum nanocrystals with high-index facets and high electro-oxidation activity. Science 2007, 316, 732-735.

[324] Li, G. R.; Hu, T.; Pan, G. L.; Yan, T. Y.; Gao, X. P.; Zhu, H. Y. Morphology-function relationship of $\mathrm{ZnO}$ : Polar planes, oxygen vacancies, and activity. J. Phys. Chem. C 2008, 112, 11859-11864.
[325] Feng, X.; Feng, L.; Jin, M.; Zhai, J.; Jiang, L.; Zhu, D. Reversible super-hydrophobicity to super-hydrophilicity transition of aligned $\mathrm{ZnO}$ nanorod films. J. Am. Chem. Soc. 2004, 126, 62-63.

[326] Onda, T.; Shibuichi, S.; Satoh, N.; Tsujii, K. Super-waterrepellent fractal surfaces. Langmuir 1996, 12, 2125-2127.

[327] Sun, R. D.; Nakajima, A.; Fujishima, A.; Watanabe, T.; Hashimoto, K. Photoinduced surface wettability conversion of $\mathrm{ZnO}$ and $\mathrm{TiO}_{2}$ thin films. J. Phys. Chem. B 2001, 105, 1984-1990.

[328] Ding, Y.; Xu, S.; Zhang, Y.; Wang, A. C.; Wang, M. H.; Xiu, Y. H.; Wong, C. P.; Wang, Z. L. Modifying the antiwetting property of butterfly wings and water strider legs by atomic layer deposition coating: surface materials versus geometry. Nanotechnology 2008, 19, 355708.

[329] Soci, C.; Zhang, A.; Xiang, B.; Dayeh, S. A.; Aplin, D. P. R.; Park, J.; Bao, X. Y.; Lo, Y. H.; Wang, D. ZnO Nanowire UV photodetectors with high internal gain. Nano Lett. 2007, 7, 1003-1009.

[330] Badre, C.; Pauporté, T. Nanostructured ZnO-based surface with reversible electrochemically adjustable wettability. Adv. Mater. 2009, 21, 697-701.

[331] Guo, M.; Diao, P.; Cai, S. M. Highly hydrophilic and superhydrophobic $\mathrm{ZnO}$ nanorod array films. Thin Solid Films 2007, 515, 7162-7166.

[332] Xu, N. S.; Huq, S. E. Novel cold cathode materials and applications. Mat. Sci. Eng. R 2005, 48, 47-189.

[333] Dev, A.; Panda, S. K.; Kar, S.; Chakrabarti, S.; Chaudhuri, S. Surfactant-assisted route to synthesize well-aligned $\mathrm{ZnO}$ nanorod arrays on sol-gel-derived $\mathrm{ZnO}$ thin films. J. Phys. Chem. B 2006, 110, 14266-14272.

[334] Hung, C. H.; Whang, W. T. Low-temperature solution approach toward highly aligned $\mathrm{ZnO}$ nanotip arrays. $J$. Cryst. Growth 2004, 268, 242-248.

[335] Cui, J. B.; Daghlian, C. P.; Gibson, U. J.; Pusche, R.; Geithner, P.; Ley, L. Low-temperature growth and field emission of $\mathrm{ZnO}$ nanowire arrays. J. Appl. Phys. 2005, 97, 044315.

[336] Wei, A.; Sun, X. W.; Xu, C. X.; Dong, Z. L.; Yu, M. B.; Huang, W. Stable field emission from hydrothermally grown ZnO nanotubes. Appl. Phys. Lett. 2006, 88, 213102.

[337] Xu, C. X.; Sun, X. W. Field emission from zinc oxide nanopins. Appl. Phys. Lett. 2003, 83, 3806-3808.

[338] Cao, B. Q.; Teng, X. M.; Heo, S. H.; Li, Y.; Cho, S. O.; Li, G. H.; Cai, W. P. Different ZnO nanostructures fabricated by a seed-layer assisted electrochemical route and their photoluminescence and field emission properties. J. Phys. Chem. C 2007, 111, 2470-2476.

[339] Ahsanulhaq, Q.; Kim, J. H.; Hahn, Y. B. Controlled selective 
growth of $\mathrm{ZnO}$ nanorod arrays and their field emission properties. Nanotechnology 2007, 18, 485307.

[340] Kee, C. S.; Ko, D. K.; Lee, J. Photonic band gaps of two-dimensional $\mathrm{ZnO}$ nanorod photonic crystals. J. Phys. D: Appl. Phys. 2005, 38, 3850-3853.

[341] Matsuu, M.; Shimada, S.; Masuya, K.; Hirano, S.; Kuwabara, M. Formation of periodically ordered zinc oxide nanopillars in aqueous solution: An approach to photonic crystals at visible wavelengths. Adv. Mater. 2006, 18, 1617-1621.

[342] Hirano, S. Oxide nanowire arrays and two-dimensional photonic crystals for control of light. J. Ceram. Soc. Jpn. 2007, 115, 92-100.

[343] Cui, J. B.; Gibson, U. Low-temperature fabrication of single-crystal $\mathrm{ZnO}$ nanopillar photonic bandgap structures. Nanotechnology 2007, 18, 155302.

[344] Cui, J. B. Structural and optical properties of periodically ordered ZnO nanowires. Sci. China Ser. E 2009, 52, 313317.

[345] Volk, J.; Hakansson, A.; Miyazaki, H. T.; Nagata, T.; Shimizu, J.; Chikyow, T. Fully engineered homoepitaxial zinc oxide nanopillar array for near-surface light wave manipulation. Appl. Phys. Lett. 2008, 92, 183114.

[346] Alivov, Y. I.; Kalinina, E. V.; Cherenkov, A. E.; Look, D. C.; Ataev, B. M.; Omaev, A. K.; Chukichev, M. V.; Bagnall, D. M. Fabrication and characterization of $n-\mathrm{ZnO} / p-\mathrm{AlGaN}$ heterojunction light-emitting diodes on $6 \mathrm{H}-\mathrm{SiC}$ substrates. Appl. Phys. Lett. 2003, 83, 4719-4721.

[347] Lyu, S. C.; Zhang, Y.; Ruh, H.; Lee, H. J.; Shim, H. W.; Suh, E. K.; Lee, C. J. Low temperature growth and photoluminescence of well-aligned zinc oxide nanowires. Chem. Phys. Lett. 2002, 363, 134-138.

[348] Tam, K. H.; Cheung, C. K.; Leung, Y. H.; Djurisic, A. B.; Ling, C. C.; Beling, C. D.; Fung, S.; Kwok, W. M.; Chan, W. K.; Phillips, D. L.; Ding, L.; Ge, W. K. Defects in ZnO nanorods prepared by a hydrothermal method. J. Phys. Chem. B 2006, 110, 20865-20871.

[349] Lucas, M.; Wang, Z. L.; Riedo, E. Combined polarized Raman and atomic force microscopy: In situ study of point defects and mechanical properties in individual $\mathrm{ZnO}$ nanobelts. Applied Physics Letts. 2009, 95, 051904.

[350] Kim, Y. J.; Shang, H. M.; Cao, G. Z. Growth and characterization of [001] $\mathrm{ZnO}$ nanorod array on ITO substrate with electric field assisted nucleation. J. Sol-Gel Sci. Technol. 2006, 38, 79-84.

[351] Zhou, H.; Chen, X. M.; Wu, G. H.; Gao, F.; Qin, N.; Bao, D. H. Significantly enhanced red photoluminescence properties of nanocomposite films composed of a ferroelectric $\mathrm{Bi}_{3.6} \mathrm{Eu}_{0.4} \mathrm{Ti}_{3} \mathrm{O}_{12}$ matrix and highly $c$-axis-oriented $\mathrm{ZnO}$ nanorods on Si substrates prepared by a hybrid chemical solution method. J. Am. Chem. Soc. 2010, 132, 1790-1791.

[352] Xiang, S.; Wang, Z. L. Unpublished results.

[353] Mahalingam, T.; Lee, K. M.; Park, K. H.; Lee, S.; Ahn, Y.; Park, J. Y.; Koh, K. H. Low temperature wet chemical synthesis of good optical quality vertically aligned crystalline ZnO nanorods. Nanotechnology 2007, 18, 035606.

[354] Hu, Y. F.; Zhang, Y.; Xu, C.; Lin, L.; Snyder, R. L.; Wang, Z. L. Self-powered system with wireless data transmission. Nano Letters 2011, 11, 2572-2577.

[355] Ho, G. W.; Wong, A. S. W. One step solution synthesis towards ultra-thin and uniform single-crystalline $\mathrm{ZnO}$ nanowires. Appl. Phys. A 2007, 86, 457-462.

[356] Li, C. C.; Chen, H. P.; Liao, H. C.; Chen, S. Y. Enhanced luminescent and electrical properties of hydrogen-plasma $\mathrm{ZnO}$ nanorods grown on wafer-scale flexible substrates. Appl. Phys. Lett. 2005, 86, 183103.

[357] Lee, S. J.; Park, S. K.; Park, C. R.; Lee, J. Y.; Park, J.; Do, Y. R. Spatially separated $\mathrm{ZnO}$ nanopillar arrays on $\mathrm{Pt} / \mathrm{Si}$ substrates prepared by electrochemical deposition. J. Phys. Chem. C 2007, 111, 11793-11801.

[358] Richters, J. P.; Voss, T.; Wischmeier, L.; Ruckmann, I.; Gutowski, J. Influence of polymer coating on the low-temperature photoluminescence properties of $\mathrm{ZnO}$ nanowires. Appl. Phys. Lett. 2008, 92, 011103.

[359] Varshni, Y. P. Temperature dependence of energy gap in semiconductors. Physica 1967, 34, 149-154.

[360] Sakai, K.; Noguchi, K.; Fukuyama, A.; Ikari, T.; Okada, T. Low-temperature photoluminescence of nanostructured $\mathrm{ZnO}$ crystal synthesized by pulsed-laser ablation. Jpn. J. Appl. Phys. 2009, 48, 085001.

[361] Su, F. H.; Wang, W. J.; Ding, K.; Li, G. H.; Liu, Y. F.; Joly, A. G.; Chen, W. Pressure dependence of the near-band-edge photoluminescence from $\mathrm{ZnO}$ microrods at low temperature. J. Phys. Chem. Solids 2006, 67, 2376-2381.

[362] Fang, F.; Zhao, D. X.; Li, B. H.; Zhang, Z. Z.; Shen, D. Z.; Wang, X. H. Bending-induced enhancement of longitudinal optical phonon scattering in $\mathrm{ZnO}$ nanowires. J. Phys. Chem. C 2010, 114, 12477-12480.

[363] Choy, J. H.; Jang, E. S.; Won, J. H.; Chung, J. H.; Jang, D. J.; Kim, Y. W. Soft solution route to directionally grown $\mathrm{ZnO}$ nanorod arrays on Si wafer; Room-temperature ultraviolet laser. Adv. Mater. 2003, 15, 1911-1914.

[364] Qiu, Z.; Wong, K. S.; Wu, M.; Lin, W.; Xu, H. Microcavity lasing behavior of oriented hexagonal $\mathrm{ZnO}$ nanowhiskers grown by hydrothermal oxidation. Appl. Phys. Lett. 2004, 84, 2739-2741.

[365] Voss, T.; Kudyk, I.; Wischmeier, L.; Gutowski, J. Nonlinear 
optics with $\mathrm{ZnO}$ nanowires. Phys. Status Solidi B 2009, 246, 311-314.

[366] Johnson, J. C.; Yan, H. Q.; Schaller, R. D.; Petersen, P. B.; Yang, P. D.; Saykally, R. J. Near-field imaging of nonlinear optical mixing in single zinc oxide nanowires. Nano Lett. 2002, 2, 279-283.

[367] Jeong, M. C.; Oh, B. Y.; Ham, M. H.; Myoung, J. M. Electroluminescence from $\mathrm{ZnO}$ nanowires in $n-\mathrm{ZnO}$ film/ZnO nanowire array $/ p$-GaN film heterojunction lightemitting diodes. Appl. Phys. Lett. 2006, 88, 202105.

[368] Jeong, M. C.; Oh, B. Y.; Ham, M. H.; Lee, S. W.; Myoung, J. M. ZnO-nanowire-inserted $\mathrm{GaN} / \mathrm{ZnO}$ heterojunction lightemitting diodes. Small 2007, 3, 568-572.

[369] Reddy, N. K.; Ahsanulhaq, Q.; Hahn, Y. B. Fabrication of zinc oxide nanorods based heterojunction devices using simple and economic chemical solution method. Appl. Phys. Lett. 2008, 93, 083124.

[370] Chen, C. H.; Chang, S. J.; Chang, S. P.; Li, M. J.; Chen, I. C.; Hsueh, T. J.; Hsu, C. L. Electroluminescence from $n$-ZnO nanowires $/ p$-GaN heterostructure light-emitting diodes. Appl. Phys. Lett. 2009, 95, 223101.

[371] Xu, S.; Xu, C.; Liu, Y.; Hu, Y.; Yang, R.; Yang, Q.; Ryou, J. H.; Kim, H. J.; Lochner, Z.; Choi, S.; Dupuis, R.; Wang, Z. L. Ordered nanowire array blue/near-UV light emitting diodes. Adv. Mater. 2010, 22, 4749-4753.

[372] Alivov, Y. I.; Van Nostrand, J. E.; Look, D. C.; Chukichev, M. V.; Ataev, B. M. Observation of $430 \mathrm{~nm}$ electroluminescence from $\mathrm{ZnO} / \mathrm{GaN}$ heterojunction light-emitting diodes. Appl. Phys. Lett. 2003, 83, 2943-2945.

[373] Bulashevich, K. A.; Evstratov, I. Y.; Karpov, S. Y. Hybrid $\mathrm{ZnO} /$ III-nitride light-emitting diodes: Modelling analysis of operation. Phys. Status Solidi A 2007, 204, 241-245.

[374] Titkov, I. E.; Zubrilov, A. S.; Delimova, L. A.; Mashovets, D. V.; Liniichuk, I. A.; Grekhov, I. V. White electroluminescence from $\mathrm{ZnO} / \mathrm{GaN}$ structures. Semiconductors 2007, 41, 564-569.

[375] Kishwar, S.; ul Hasan, K.; Tzamalis, G.; Nur, O.; Willander, M.; Kwack, H. S.; Dang, D. L. S. Electro-optical and cathodoluminescence properties of low temperature grown $\mathrm{ZnO}$ nanorods $/ p$-GaN white light emitting diodes. Phys. Status Solidi A 2010, 207, 67-72.

[376] Guo, R.; Nishimura, J.; Matsumoto, M.; Higashihata, M.; Nakamura, D.; Okada, T. Electroluminescence from $\mathrm{ZnO}$ nanowire-based $p$-GaN $/ n-\mathrm{ZnO}$ heterojunction light-emitting diodes. Appl. Phys. B 2009, 94, 33-38.

[377] Xu, H. Y.; Liu, Y. C.; Liu, Y. X.; Xu, C. S.; Shao, C. L.; Mu, R. Ultraviolet electroluminescence from $p-\mathrm{GaN} / i-\mathrm{ZnO} / n-\mathrm{ZnO}$ heterojunction light-emitting diodes. Appl. Phys. B 2005, 80, 871-874.
[378] Liu, H. F.; Hu, G. X.; Gong, H.; Zang, K. Y.; Chua, S. J. Effects of oxygen on low-temperature growth and band alignment of $\mathrm{ZnO} / \mathrm{GaN}$ heterostructures. J. Vac. Sci. Technol. A 2008, 26, 1462-1468.

[379] Aguilar, C. A.; Haight, R.; Mavrokefalos, A.; Korgel, B. A.; Chen, S. C. Probing electronic properties of molecular engineered zinc oxide nanowires with photoelectron spectroscopy. ACS Nano 2009, 3, 3057-3062.

[380] Ng, A. M. C.; Xi, Y. Y.; Hsu, Y. F.; Djurisic, A. B.; Chan, W. K.; Gwo, S.; Tam, H. L.; Cheah, K. W.; Fong, P. W. K.; Lui, H. F.; Surya, C. GaN/ZnO nanorod light emitting diodes with different emission spectra. Nanotechnology 2009, 20, 445201

[381] Alivov, Y. I.; Ozgur, U.; Dogan, S.; Liu, C.; Moon, Y.; Gu, X.; Avrutin, V.; Fu, Y.; Morkoc, H. Forward-current electroluminescence from $\mathrm{GaN} / \mathrm{ZnO}$ double heterostructure diode. Solid-State Electron. 2005, 49, 1693-1696.

[382] Zhang, X. M.; Lu, M. Y.; Zhang, Y.; Chen, L. J.; Wang, Z. L. Fabrication of a high-brightness blue-light-emitting diode using a $\mathrm{ZnO}$-nanowire array grown on $p$-GaN thin film. Adv. Mater. 2009, 21, 2767-2770.

[383] Bayram, C.; Teherani, F. H.; Rogers, D. J.; Razeghi, M. A hybrid green light-emitting diode comprised of $n-\mathrm{ZnO} /$ (InGaN/GaN) multi-quantum-wells/p-GaN. Appl. Phys. Lett. 2008, 93, 081111.

[384] Choi, H. W.; Jeon, C. W.; Dawson, M. D.; Edwards, P. R.; Martin, R. W.; Tripathy, S. Mechanism of enhanced light output efficiency in InGaN-based microlight emitting diodes. J. Appl. Phys. 2003, 93, 5978-5982.

[385] Konenkamp, R.; Word, R. C.; Schlegel, C. Vertical nanowire light-emitting diode. Appl. Phys. Lett. 2004, 85, 6004-6006.

[386] Konenkamp, R.; Word, R. C.; Godinez, M. Ultraviolet electroluminescence from $\mathrm{ZnO}$ /polymer heterojunction light-emitting diodes. Nano Lett. 2005, 5, 2005-2008.

[387] Bolink, H. J.; Coronado, E.; Repetto, D.; Sessolo, M. Air stable hybrid organic-inorganic light emitting diodes using $\mathrm{ZnO}$ as the cathode. Appl. Phys. Lett. 2007, 91, 223501.

[388] Konenkamp, R.; Nadarajah, A.; Word, R. C.; Meiss, J.; Engelhardt, R. ZnO nanowires for LED and field-emission displays. J. Soc. Inf. Display 2008, 16, 609-613.

[389] Willander, M.; Lozovik, Y. E.; Wadeasa, A.; Nur, O.; Semenov, A. G.; Vonorova, N. S. Light emission from different $\mathrm{ZnO}$ junctions and nanostructures. Phys. Status Solidi A 2009, 206, 853-859.

[390] Wadeasa, A.; Beegum, S. L.; Raja, S.; Nur, O.; Willander, M. The demonstration of hybrid $n-\mathrm{ZnO}$ nanorod $/ p$-polymer heterojunction light emitting diodes on glass substrates. Appl. Phys. A 2009, 95, 807-812.

[391] Guo, H. G.; Zhou, J. Z.; Lin, Z. G. ZnO nanorod light- 
emitting diodes fabricated by electrochemical approaches. Electrochem. Commun. 2008, 10, 146-150.

[392] Liu, J.; Ahn, Y. H.; Park, J. Y.; Koh, K. H.; Lee, S. Hybrid light-emitting diodes based on flexible sheets of massproduced ZnO nanowires. Nanotechnology 2009, 20, 445203.

[393] Sun, X. W.; Huang, J. Z.; Wang, J. X.; Xu, Z. A ZnO nanorod inorganic/organic heterostructure light-emitting diode emitting at $342 \mathrm{~nm}$. Nano Lett. 2008, 8, 1219-1223.

[394] Round, H. J. A note on carborundum. Electrical World 1907, 49, 309.

[395] Livingst. A. W.; Turvey, K.; Allen, J. W. Electroluminescence in forward-biased zinc selenide Schottky diodes. Solid-State Electron. 1973, 16, 351-356.

[396] Allen, J. W.; Livingstone, A. W.; Turvey, K. Electroluminescence in reverse-biased zinc selenide Schottky diodes. Solid-State Electron. 1972, 15, 1363-1369.

[397] Lazarouk, S.; Jaguiro, P.; Katsouba, S.; Masini, G.; LaMonica, S.; Maiello, G.; Ferrari, A. Stable electroluminescence from reverse biased $n$-type porous silicon-aluminum Schottky junction device. Appl. Phys. Lett. 1996, 68, 2108-2110.

[398] Steckl, A. J.; Garter, M.; Birkhahn, R.; Scofield, J. Green electroluminescence from Er-doped GaN Schottky barrier diodes. Appl. Phys. Lett. 1998, 73, 2450-2452.

[399] Wang, Y. X.; Zhang, Q. F.; Sun, H.; Chang, Y. L.; Wu, J. L. Fabrication of $\mathrm{ZnO}$ nanowire-based diodes and their light-emitting properties. Acta Phys. Sin.-Chin. Ed. 2008, 57, 1141-1144.

[400] Guo, H. H.; Lin, Z. H.; Feng, Z. F.; Lin, L. L.; Zhou, J. Z. White-light-emitting diode based on $\mathrm{ZnO}$ nanotubes. $J$. Phys. Chem. C 2009, 113, 12546-12550.

[401] Bano, N.; Hussain, I.; Nur, O.; Willander, M.; Kwack, H. S.; Dang, D. L. S. Study of $\mathrm{Au} / \mathrm{ZnO}$ nanorods Schottky light-emitting diodes grown by low-temperature aqueous chemical method. Appl. Phys. A 2010, 100, 467-472.

[402] Tan, S. T.; Sun, X. W.; Zhao, J. L.; Iwan, S.; Cen, Z. H.; Chen, T. P.; Ye, J. D.; Lo, G. Q.; Kwong, D. L.; Teo, K. L. Ultraviolet and visible electroluminescence from $n-\mathrm{ZnO} / \mathrm{SiO}_{x} /(n, p)$-Si heterostructured light-emitting diodes. Appl. Phys. Lett. 2008, 93, 013506.

[403] Benisty, H.; De Neve, H.; Weisbuch, C. Impact of planar microcavity effects on light extraction-Part I: Basic concepts and analytical trends. IEEE J. Quantum. Elect. 1998, 34, 1612-1631.

[404] Schnitzer, I.; Yablonovitch, E.; Caneau, C.; Gmitter, T. J.; Scherer, A. $30 \%$ external quantum efficiency from surface textured, thin-film light-emitting-diodes. Appl. Phys. Lett. 1993, 63, 2174-2176.

[405] Fujii, T.; Gao, Y.; Sharma, R.; Hu, E. L.; DenBaars, S. P.; Nakamura, S. Increase in the extraction efficiency of
GaN-based light-emitting diodes via surface roughening. Appl. Phys. Lett. 2004, 84, 855-857.

[406] Kim, H.; Kim, K. K.; Choi, K. K.; Kim, H.; Song, J. O.; Cho, J.; Baik, K. H.; Sone, C.; Park, Y.; Seong, T. Y. Design of high-efficiency GaN-based light emitting diodes with vertical injection geometry. Appl. Phys. Lett. 2007, 91, 023510.

[407] Murai, A.; Thompson, D. B.; Masui, H.; Fellows, N.; Mishra, U. K.; Nakamura, S.; DenBaars, S. P. Hexagonal pyramid shaped light-emitting diodes based on $\mathrm{ZnO}$ and $\mathrm{GaN}$ direct wafer bonding. Appl. Phys. Lett. 2006, 89, 171116.

[408] Schubert, E. F.; Kim, J. K. Solid-state light sources getting smart. Science 2005, 308, 1274-1278.

[409] Barnes, W. L. Electromagnetic crystals for surface plasmon polaritons and the extraction of light from emissive devices. J. Lightwave Technol. 1999, 17, 2170-2182.

[410] Chen, L.; Nurmikko, A. V. Fabrication and performance of efficient blue light emitting III-nitride photonic crystals. Appl. Phys. Lett. 2004, 85, 3663-3665.

[411] Oder, T. N.; Kim, K. H.; Lin, J. Y.; Jiang, H. X. III-Nitride blue and ultraviolet photonic crystal light emitting diodes. Appl. Phys. Lett. 2004, 84, 466-468.

[412] Lu, Y.; Zhong, J.; Zhu, J.; Saraf, G.; Chen, H. H.; Duan, Z. Q.; Reyes, P.; Shen, H.; Mackie, D. M.; Wittkstruck, R. H.; Ballato, A. Novel devices using multifunctional $\mathrm{ZnO}$ and its nanostructures. http://dodreports.com/pdf/ada505710.pdf.

[413] Kim, K. K.; Lee, S. D.; Kim, H.; Park, J. C.; Lee, S. N.; Park, Y.; Park, S. J.; Kim, S. W. Enhanced light extraction efficiency of GaN-based light-emitting diodes with $\mathrm{ZnO}$ nanorod arrays grown using aqueous solution. Appl. Phys. Lett. 2009, 94, 071118.

[414] An, S. J.; Chae, J. H.; Yi, G. C.; Park, G. H. Enhanced light output of GaN-based light-emitting diodes with $\mathrm{ZnO}$ nanorod arrays. Appl. Phys. Lett. 2008, 92, 121108.

[415] Zhong, J.; Chen, H.; Saraf, G.; Lu, Y.; Choi, C. K.; Song, J. J.; Mackie, D. M.; Shen, H. Integrated ZnO nanotips on GaN light emitting diodes for enhanced emission efficiency. Appl. Phys. Lett. 2007, 90, 203515.

[416] Lai, E.; Kim, W.; Yang, P. Vertical nanowire array-based light emitting diodes. Nano Res. 2008, 1, 123-128.

[417] Bao, J. M.; Zimmler, M. A.; Capasso, F.; Wang, X. W.; Ren, Z. F. Broadband $\mathrm{ZnO}$ single-nanowire light-emitting diode. Nano Lett. 2006, 6, 1719-1722.

[418] Johnson, J. C.; Yan, H. Q.; Yang, P. D.; Saykally, R. J. Optical cavity effects in $\mathrm{ZnO}$ nanowire lasers and waveguides. J. Phys. Chem. B 2003, 107, 8816-8828.

[419] Kim, K. S.; Kim, S. M.; Jeong, H.; Jeong, M. S.; Jung, G. Y. Enhancement of light extraction through the wave-guiding effect of $\mathrm{ZnO}$ sub-microrods in InGaN blue light-emitting 
diodes. Adv. Funct. Mater. 2010, 20, 1076-1082.

[420] Voss, T.; Svacha, G. T.; Mazur, E.; Muller, S.; Ronning, C.; Konjhodzic, D.; Marlow, F. High-order waveguide modes in $\mathrm{ZnO}$ nanowires. Nano Letters. 2007, 7, 3675-3680.

[421] Park, S. H.; Kim, S. H.; Han, S. W. Growth of homoepitaxial $\mathrm{ZnO}$ film on $\mathrm{ZnO}$ nanorods and light emitting diode applications. Nanotechnology 2007, 18, 055608.

[422] Sun, X. W.; Wang, J. X. Fast switching electrochromic display using a viologen-modified $\mathrm{ZnO}$ nanowire array electrode. Nano Lett. 2008, 8, 1884-1889.

[423] Granqvist, C. G. Electrochromic tungsten oxide films: Review of progress 1993-1998. Sol. Energy Mater. Sol. Cells 2000, 60, 201-262.

[424] Bonhote, P.; Gogniat, E.; Campus, F.; Walder, L.; Gratzel, M. Nanocrystalline electrochromic displays. Displays 1999, 20, 137-144.

[425] Hu, A. Z.; Wu, F.; Liu, J. P.; Jiang, J.; Ding, R. M.; Li, X.; Cheng, C. X.; Zhu, Z. H.; Huang, X. T. Density- and adhesion-controlled $\mathrm{ZnO}$ nanorod arrays on the ITO flexible substrates and their electrochromic performance. $J$. Alloys Compd. 2010, 507, 261-266.

[426] Li, M.; Zhang, H. Y.; Guo, C. X.; Xu, J. B.; Fu, X. J. The research on suspended $\mathrm{ZnO}$ nanowire field-effect transistor. Chin. Phys. B 2009, 18, 1594-1597.

[427] Sun, B. Q.; Sirringhaus, H. Surface tension and fluid flow driven self-assembly of ordered $\mathrm{ZnO}$ nanorod films for high-performance field effect transistors. J. Am. Chem. Soc. 2006, 128, 16231-16237.

[428] Ju, S. Y.; Facchetti, A.; Xuan, Y.; Liu, J.; Ishikawa, F.; Ye, P. D.; Zhou, C. W.; Marks, T. J.; Janes, D. B. Fabrication of fully transparent nanowire transistors for transparent and flexible electronics. Nat. Nanotech. 2007, 2, 378-384.

[429] Hsu, C. L.; Tsai, T. Y. Fabrication of fully transparent indium-doped $\mathrm{ZnO}$ nanowire field-effect transistors on ITO/glass substrates. J. Electrochem. Soc. 2011, 158, $\mathrm{K} 20-\mathrm{K} 23$.

[430] Ko, S. H.; Park, I.; Pan, H.; Misra, N.; Rogers, M. S.; Grigoropoulos, C. P.; Pisano, A. P. ZnO nanowire network transistor fabrication on a polymer substrate by lowtemperature, all-inorganic nanoparticle solution process. Appl. Phys. Lett. 2008, 92, 154102.

[431] Gao, P. X.; Liu, J.; Buchine, B. A.; Weintraub, B.; Wang, Z. L.; Lee, J. L. Bridged $\mathrm{ZnO}$ nanowires across trenched electrodes. Appl. Phys. Lett. 2007, 91, 142108.

[432] Xu, S.; Qin, Y.; Xu, C.; Wei, Y. G.; Yang, R. S.; Wang, Z. L. Self-powered nanowire devices. Nat. Nanotechnol. 2010, $5,366-373$.

[433] Wang, J. X.; Sun, X. W.; Yang, Y.; Huang, H.; Lee, Y. C.; Tan, O. K.; Vayssieres, L. Hydrothermally grown oriented
$\mathrm{ZnO}$ nanorod arrays for gas sensing applications. Nanotechnology 2006, 17, 4995-4998.

[434] Sun, K.; Jing, Y.; Park, N.; Li, C.; Bando, Y.; Wang, D. L. Solution synthesis of large-scale, high-sensitivity $\mathrm{ZnO} / \mathrm{Si}$ hierarchical nanoheterostructure photodetectors. J. Am. Chem. Soc. 2010, 132, 15465-15467.

[435] Gao, P.; Wang, Z. Z.; Liu, K. H.; Xu, Z.; Wang, W. L.; Bai, X. D.; Wang, E. G. Photoconducting response on bending of individual $\mathrm{ZnO}$ nanowires. J. Mater. Chem. 2009, 19, 1002-1005.

[436] Wei, A.; Sun, X. W.; Wang, J. X.; Lei, Y.; Cai, X. P.; Li, C. M.; Dong, Z. L.; Huang, W. Enzymatic glucose biosensor based on $\mathrm{ZnO}$ nanorod array grown by hydrothermal decomposition. Appl. Phys. Lett. 2006, 89, 123902.

[437] Liu, N. S.; Fang, G. J.; Zeng, W.; Long, H.; Yuan, L. Y.; Zhao, X. Z. Novel ZnO nanorod flexible strain sensor and strain friving transistor with an ultrahigh $10^{7}$ scale "On""Off" ratio fabricated by a single-step hydrothermal reaction. J. Phys. Chem. C 2011, 115, 570-575.

[438] Kwon, S. S.; Hong, W. K.; Jo, G.; Maeng, J.; Kim, T. W.; Song, S.; Lee, T. Piezoelectric effect on the electronic transport characteristics of $\mathrm{ZnO}$ nanowire field-effect transistors on bent flexible substrates. Adv. Mater. 2008, 20, 4557-4562.

[439] Wei, T. Y.; Huang, C. T.; Hansen, B. J.; Lin, Y. F.; Chen, L. J.; Lu, S. Y.; Wang, Z. L. Large enhancement in photon detection sensitivity via Schottky-gated $\mathrm{CdS}$ nanowire nanosensors. Appl. Phys. Lett. 2010, 96, 013508.

[440] Al-Hilli, S. M.; Willander, M.; Ost, A.; Stralfors, P. ZnO nanorods as an intracellular sensor for $\mathrm{pH}$ measurements. $J$. Appl. Phys. 2007, 102, 084304.

[441] Zhou, J.; Xu, N. S.; Wang, Z. L. Dissolving behavior and stability of $\mathrm{ZnO}$ wires in biofluids: A study on biodegradability and biocompatibility of $\mathrm{ZnO}$ nanostructures. Adv. Mater. 2006, 18, 2432-2435.

[442] Li, Z.; Yang, R. S.; Yu, M.; Bai, F.; Li, C.; Wang, Z. L. Cellular level biocompatibility and biosafety of $\mathrm{ZnO}$ nanowires. J. Phys. Chem. C 2008, 112, 20114-20117.

[443] Gonzalez-Valls, I.; Lira-Cantu, M. Vertically-aligned nanostructures of $\mathrm{ZnO}$ for excitonic solar cells: A review. Energy Environ. Sci. 2009, 2, 19-34.

[444] Lee, Y. J.; Ruby, D. S.; Peters, D. W.; McKenzie, B. B.; Hsu, J. W. P. ZnO nanostructures as efficient antireflection layers in solar cells. Nano Lett. 2008, 8, 1501-1505.

[445] O'Regan, B.; Gratzel, M. A Low-cost, high-efficiency solarcell based on dye-sensitized colloidal $\mathrm{TiO}_{2}$ films. Nature 1991, 353, 737-740.

[446] Pradhan, B.; Batabyal, S. K.; Pal, A. J. Vertically aligned 
$\mathrm{ZnO}$ nanowire arrays in Rose Bengal-based dye-sensitized solar cells. Sol. Energy Mater. Sol. Cells 2007, 91, 769-773.

[447] Baxter, J. B.; Aydil, E. S. Nanowire-based dye-sensitized solar cells. Appl. Phys. Lett. 2005, 86, 053114.

[448] Beermann, N.; Vayssieres, L.; Lindquist, S. E.; Hagfeldt, A. Photoelectrochemical studies of oriented nanorod thin films of hematite. J. Electrochem. Soc. 2000, 147, 24562461.

[449] Baxter, J. B.; Schmuttenmaer, C. A. Conductivity of ZnO nanowires, nanoparticles, and thin films using time-resolved terahertz spectroscopy. J. Phys. Chem. B 2006, 110, 25229-25239.

[450] Martinson, A. B. F.; McGarrah, J. E.; Parpia, M. O. K.; Hupp, J. T. Dynamics of charge transport and recombination in $\mathrm{ZnO}$ nanorod array dye-sensitized solar cells. Phys. Chem. Chem. Phys. 2006, 8, 4655-4659.

[451] Baxter, J. B.; Walker, A. M.; van Ommering, K.; Aydil, E. S Synthesis and characterization of $\mathrm{ZnO}$ nanowires and their integration into dye-sensitized solar cells. Nanotechnology 2006, 17, S304-S312.

[452] Hsu, Y. F.; Xi, Y. Y.; Djurisic, A. B.; Chan, W. K. ZnO nanorods for solar cells: Hydrothermal growth versus vapor deposition. Appl. Phys. Lett. 2008, 92, 133507.

[453] Jiang, C. Y.; Sun, X. W.; Tan, K. W.; Lo, G. Q.; Kyaw, A. K. K.; Kwong, D. L. High-bendability flexible dye-sensitized solar cell with a nanoparticle-modified $\mathrm{ZnO}$-nanowire electrode. Appl. Phys. Lett. 2008, 92, 143101.

[454] Leschkies, K. S.; Divakar, R.; Basu, J.; Enache-Pommer, E.; Boercker, J. E.; Carter, C. B.; Kortshagen, U. R.; Norris, D. J.; Aydil, E. S. Photosensitization of $\mathrm{ZnO}$ nanowires with CdSe quantum dots for photovoltaic devices. Nano Lett. 2007, 7, 1793-1798.

[455] Han, J. B.; Fan, F. R.; Xu, C.; Lin, S. S.; Wei, M.; Duan, X.; Wang, Z. L. ZnO nanotube-based dye-sensitized solar cell and its application in self-powered devices. Nanotechnology 2010, 21, 405203.

[456] Jensen, R. A.; Van Ryswyk, H.; She, C. X.; Szarko, J. M.; Chen, L. X.; Hupp, J. T. Dye-sensitized solar cells: Sensitizerdependent injection into $\mathrm{ZnO}$ nanotube electrodes. Langmuir 2010, 26, 1401-1404.

[457] Yodyingyong, S.; Zhang, Q. F.; Park, K.; Dandeneau, C. S.; Zhou, X. Y.; Triampo, D.; Cao, G. Z. ZnO nanoparticles and nanowire array hybrid photoanodes for dye-sensitized solar cells. Appl. Phys. Lett. 2010, 96, 073115.

[458] Ku, C. H.; Wu, J. J. Chemical bath deposition of $\mathrm{ZnO}$ nanowire-nanoparticle composite electrodes for use in dyesensitized solar cells. Nanotechnology 2007, 18, 505706.

[459] Cheng, K.; Cheng, G.; Wang, S. J.; Fu, D. W.; Zou, B. S.; $\mathrm{Du}, \mathrm{Z}$. L. Electron transport properties in $\mathrm{ZnO}$ nanowires/ poly(3-hexylthiophene) hybrid nanostructure. Mater. Chem. Phys. 2010, 124, 1239-1242.

[460] Ku, C. H.; Wua, J. J. Electron transport properties in $\mathrm{ZnO}$ nanowire array/nanoparticle composite dye-sensitized solar cells. Appl. Phys. Lett. 2007, 91, 093117.

[461] Jiang, C. Y.; Sun, X. W.; Lo, G. Q.; Kwong, D. L.; Wang, J. X. Improved dye-sensitized solar cells with a $\mathrm{ZnO}-$ nanoflower photoanode. Appl. Phys. Lett. 2007, 90, 263501.

[462] Hosono, E.; Fujihara, S.; Honna, I.; Zhou, H. S. The fabrication of an upright-standing zinc oxide nanosheet for use in dye-sensitized solar cells. Adv. Mater. 2005, 17, 2091-2094.

[463] Xu, F.; Dai, M.; Lu, Y. N.; Sun, L. T. Hierarchical ZnO nanowire-nanosheet architectures for high power conversion efficiency in dye-sensitized solar cells. J. Phys. Chem. C 2010, 114, 2776-2782.

[464] Fu, Y. S.; Sun, J.; Xie, Y.; Liu, J.; Wang, H. L.; Du, X. W. $\mathrm{ZnO}$ hierarchical nanostructures and application on highefficiency dye-sensitized solar cells. Mater. Sci. Eng., B 2010, 166, 196-202.

[465] Gao, Y. F.; Nagai, M. Morphology evolution of $\mathrm{ZnO}$ thin films from aqueous solutions and their application to solar cells. Langmuir 2006, 22, 3936-3940.

[466] Zou, D. C.; Wang, D.; Chu, Z. Z.; Lv, Z. B.; Fan, X. Fiber-shaped flexible solar cells. Coord. Chem. Rev. 2010, 254, 1169-1178.

[467] Wu, J. J.; Chen, G. R.; Yang, H. H.; Ku, C. H.; Lai, J. Y. Effects of dye adsorption on the electron transport properties in ZnO-nanowire dye-sensitized solar cells. Appl. Phys. Lett. 2007, 90, 213109.

[468] Zhang, Q. F.; Dandeneau, C. S.; Zhou, X. Y.; Cao, G. Z. $\mathrm{ZnO}$ nanostructures for dye-sensitized solar cells. Adv. Mater. 2009, 21, 4087-4108.

[469] Tak, Y.; Kim, H.; Lee, D.; Yong, K. Type-II CdS nanoparticle-ZnO nanowire heterostructure arrays fabricated by a solution process: Enhanced photocatalytic activity. Chem. Commun. 2008, 4585-4587.

[470] Lee, M.; Yang, R.; Li, C.; Wang, Z. L. Nanowire-quantum dot hybridized cell for harvesting sound and solar energies. J. Phys. Chem. Lett. 2010, 1, 2929-2935.

[471] Tena-Zaera, R.; Katty, A.; Bastide, S.; Levy-Clement, C. Annealing effects on the physical properties of electrodeposited $\mathrm{ZnO} / \mathrm{CdSe}$ core-shell nanowire arrays. Chem. Mater. 2007, 19, 1626-1632.

[472] Zhang, Y.; Xie, T. F.; Jiang, T. F.; Wei, X.; Pang, S.; Wang, X.; Wang, D. Surface photovoltage characterization of a $\mathrm{ZnO}$ nanowire array/CdS quantum dot heterogeneous film and its application for photovoltaic devices. Nanotechnology 2009, 20, 155707. 
[473] Schaller, R. D.; Klimov, V. I. High efficiency carrier multiplication in PbSe nanocrystals: Implications for solar energy conversion. Phys. Rev. Lett. 2004, 92, 186601.

[474] Kongkanand, A.; Tvrdy, K.; Takechi, K.; Kuno, M.; Kamat, P. V. Quantum dot solar cells. Tuning photoresponse through size and shape control of $\mathrm{CdSe}-\mathrm{TiO}_{2}$ architecture. J. Am. Chem. Soc. 2008, 130, 4007-4015.

[475] Tak, Y.; Hong, S. J.; Lee, J. S.; Yong, K. Fabrication of $\mathrm{ZnO} / \mathrm{CdS}$ core/shell nanowire arrays for efficient solar energy conversion. J. Mater. Chem. 2009, 19, 5945-5951.

[476] Consonni, V.; Rey, G.; Bonaime, J.; Karst, N.; Doisneau, B.; Roussel, H.; Renet, S.; Bellet, D. Synthesis and physical properties of $\mathrm{ZnO} / \mathrm{CdTe}$ core shell nanowires grown by low-cost deposition methods. Appl. Phys. Lett. 2011, 98, 111906.

[477] Sun, X. W.; Chen, J.; Song, J. L.; Zhao, D. W.; Deng, W. Q.; Lei, W. Ligand capping effect for dye solar cells with a CdSe quantum dot sensitized $\mathrm{ZnO}$ nanorod photoanode. Opt. Express 2010, 18, 1296-1301.

[478] Cui, J.; Gibson, U. J. A simple two-step electrodeposition of $\mathrm{Cu}_{2} \mathrm{O} / \mathrm{ZnO}$ nanopillar solar cells. J. Phys. Chem. C 2010, 114, 6408-6412.

[479] Zhao, Q. D.; Xie, T. F.; Peng, L. L.; Lin, Y. H.; Wang, P.; Peng, L.; Wang, D. J. Size- and orientation-dependent photovoltaic properties of $\mathrm{ZnO}$ nanorods. J. Phys. Chem. C 2007, 111, 17136-17145.

[480] Tena-Zaera, R.; Ryan, M. A.; Katty, A.; Hodes, G.; Bastide, S.; Lévy-Clément, C. Fabrication and characterization of $\mathrm{ZnO}$ nanowires/CdSe/CuSCN eta-solar cell. C.R. Chim. 2006, 9, 717-729.

[481] Olson, D. C.; Piris, J.; Collins, R. T.; Shaheen, S. E.; Ginley, D. S. Hybrid photovoltaic devices of polymer and $\mathrm{ZnO}$ nanofiber composites. Thin Solid Films 2006, 496, 26-29.

[482] Takanezawa, K.; Tajima, K.; Hashimoto, K. Efficiency enhancement of polymer photovoltaic devices hybridized with $\mathrm{ZnO}$ nanorod arrays by the introduction of a vanadium oxide buffer layer. Appl. Phys. Lett. 2008, 93, 063308.

[483] Unalan, H. E.; Hiralal, P.; Kuo, D.; Parekh, B.; Amaratunga, G.; Chhowalla, M. Flexible organic photovoltaics from zinc oxide nanowires grown on transparent and conducting single walled carbon nanotube thin films. J. Mater. Chem. 2008, 18, 5909-5912.

[484] Liu, J.; Wang, S.; Bian, Z.; Shan, M.; Huang, C. Organic/ inorganic hybrid solar cells with vertically oriented $\mathrm{ZnO}$ nanowires. Appl. Phys. Lett. 2009, 94, 173107.

[485] Takanezawa, K.; Hirota, K.; Wei, Q. S.; Tajima, K.; Hashimoto, K. Efficient charge collection with $\mathrm{ZnO}$ nanorod array in hybrid photovoltaic devices. J. Phys. Chem. C 2007, $111,7218-7223$.
[486] Briseno, A. L.; Holcombe, T. W.; Boukai, A. I.; Garnett, E. C.; Shelton, S. W.; Frechet, J. J. M.; Yang, P. D. Oligoand polythiophene/ZnO hybrid nanowire solar cells. Nano Lett. 2010, 10, 334-340.

[487] Ravirajan, P.; Peiró, A. M.; Nazeeruddin, M. K.; Graetzel, M.; Bradley, D. D. C.; Durrant, J. R.; Nelson, J. Hybrid polymer/zinc oxide photovoltaic devices with vertically oriented $\mathrm{ZnO}$ nanorods and an amphiphilic molecular interface layer. J. Phys. Chem. B 2006, 110, 7635-7639.

[488] Bi, D. Q.; Wu, F.; Qu, Q. Y.; Yue, W. J.; Cui, Q.; Shen, W.; Chen, R. Q.; Liu, C. W.; Qiu, Z. L.; Wang, M. T. Device performance related to amphiphilic modification at charge separation interface in hybrid solar cells with vertically aligned ZnO nanorod arrays. J. Phys. Chem. C 2011, 115, 3745-3752.

[489] Olson, D. C.; Lee, Y. J.; White, M. S.; Kopidakis, N.; Shaheen, S. E.; Ginley, D. S.; Voigt, J. A.; Hsu, J. W. P. Effect of polymer processing on the performance of poly(3-hexylthiophene)/ZnO nanorod photovoltaic devices. J. Phys. Chem. C 2007, 111, 16640-16645.

[490] Olson, D. C.; Shaheen, S. E.; Collins, R. T.; Ginley, D. S. The effect of atmosphere and $\mathrm{ZnO}$ morphology on the performance of hybrid poly(3-hexylthiophene)/ZnO nanofiber photovoltaic devices. J. Phys. Chem. C 2007, 111, 1667016678.

[491] Peiro, A. M.; Ravirajan, P.; Govender, K.; Boyle, D. S.; O'Brien, P.; Bradley, D. D. C.; Nelson, J.; Durrant, J. R. Hybrid polymer/metal oxide solar cells based on $\mathrm{ZnO}$ columnar structures. J. Mater. Chem. 2006, 16, 2088-2096.

[492] Lin, Y. Y.; Chen, C. W.; Chu, T. H.; Su, W. F.; Lin, C. C.; $\mathrm{Ku}, \mathrm{C} . \mathrm{H} . ; \mathrm{Wu}$, J. J.; Chen, C. H. Nanostructured metal oxide/conjugated polymer hybrid solar cells by low temperature solution processes. J. Mater. Chem. 2007, 17, 4571-4576.

[493] Greene, L. E.; Law, M.; Yuhas, B. D.; Yang, P. D. ZnO$\mathrm{TiO}_{2}$ core-shell nanorod/P3HT solar cells. J. Phys. Chem. C 2007, 111, 18451-18456.

[494] Liu, J. P.; Qu, S. C.; Xu, Y.; Chen, Y. H.; Zeng, X. B.; Wang, Z. J.; Zhou, H. Y.; Wang, Z. G. Photovoltaic and electroluminescence characters in hybrid $\mathrm{ZnO}$ and conjugated polymer bulk heterojunction devices. Chin. Phys. Lett. 2007, 24, 1350-1353.

[495] Wang, Z. L. Self-powered nanotech-Nanosize machines need still tinier power plants. Sci. Am. 2008, 298, 82-87.

[496] Tian, B. Z.; Zheng, X. L.; Kempa, T. J.; Fang, Y.; Yu, N. F.; Yu, G. H.; Huang, J. L.; Lieber, C. M. Coaxial silicon nanowires as solar cells and nanoelectronic power sources. Nature 2007, 449, 885-890.

[497] Pan, C. F.; Wu, H.; Wang, C.; Wang, B.; Zhang, L.; Cheng, 
Z. D.; Hu, P.; Pan, W.; Zhou, Z. Y.; Yang, X.; Zhu, J. Nanowire-based high performance "micro fuel cell": One nanowire, one fuel cell. Adv. Mater. 2008, 20, 1644-1648.

[498] Hudak, N. S.; Amatucci, G. G. Small-scale energy harvesting through thermoelectric, vibration, and radiofrequency power conversion. J. Appl. Phys. 2008, 103, 101301.

[499] Choi, M. Y.; Choi, D.; Jin, M. J.; Kim, I.; Kim, S. H.; Choi, J. Y.; Lee, S. Y.; Kim, J. M.; Kim, S. W. Mechanically powered transparent flexible charge-generating nanodevices with piezoelectric $\mathrm{ZnO}$ nanorods. Adv. Mater. 2009, 21, 2185-2189.

[500] Lei, Y.; Jiao, Z.; Wu, M. H.; Wilde, G. Ordered arrays of nanostructures and applications in high-efficient nanogenerators. Adv. Eng. Mater. 2007, 9, 343-348.

[501] Su, W. S.; Chen, Y. F.; Hsiao, C. L.; Tu, L. W. Generation of electricity in GaN nanorods induced by piezoelectric effect. Appl. Phys. Lett. 2007, 90, 063110.

[502] Lu, M. T.; Song, J. H.; Lu, M. P.; Lee, C. Y.; Chen, L. J.; Wang, Z. L. ZnO-ZnS heterojunction and $\mathrm{ZnS}$ nanowire arrays for electricity generation. ACS Nano 2009, 3, 357-362.

[503] Huang, C. T.; Song, J. H.; Lee, W. F.; Ding, Y.; Gao, Z. Y.; Hao, Y.; Chen, L. J.; Wang, Z. L. GaN nanowire arrays for high-output nanogenerators. J. Am. Chem. Soc. 2010, 132, 4766-4771.

[504] Huang, C. T.; Song, J. H.; Tsai, C. M.; Lee, W. F.; Lien, D. H.; Gao, Z. Y.; Hao, Y.; Chen, L. J.; Wang, Z. L. Single-InN-nanowire nanogenerator with up to $1 \mathrm{~V}$ output voltage. Adv. Mater. 2010, 22, 4008-4013.

[505] Wang, X. B.; Song, J. H.; Zhang, F.; He, C. Y.; Hu, Z.; Wang, Z. L. Electricity generation based on one-dimensional group-III nitride nanomaterials. Adv. Mater. 2010, 22, 21552158.

[506] Lin, Y. F.; Song, J.; Ding, Y.; Lu, S. Y.; Wang, Z. L. Alternating the output of a $\mathrm{CdS}$ nanowire nanogenerator by a white-light-stimulated optoelectronic effect. $A d v$. Mater. 2008, 20, 3127-3130.

[507] Lu, M. Y.; Song, J. H.; Lu, M. P.; Lee, C. Y.; Chen, L. J.; Wang, Z. L. ZnO-ZnS heterojunction and $\mathrm{ZnS}$ nanowire arrays for electricity generation. ACS Nano 2009, 3, 357362.

[508] Chen, X.; Xu, S.; Yao, N.; Shi, Y. 1.6 V Nanogenerator for mechanical energy harvesting using PZT nanofibers. Nano Lett. 2010, 10, 2133-2137.

[509] Qi, Y.; Jafferis, N. T.; Lyons, K.; Lee, C. M.; Ahmad, H.; McAlpine, M. C. Piezoelectric ribbons printed onto rubber for flexible energy conversion. Nano Lett. 2010, 10, 524528.

[510] Qi, Y.; McAlpine, M. C. Nanotechnology-enabled flexible and biocompatible energy harvesting. Energy Environ. Sci.
2010, 3, 1275-1285.

[511] Feng, X.; Yang, B. D.; Liu, Y. M.; Wang, Y.; Dagdeviren, C.; Liu, Z. J.; Carlson, A.; Li, J. Y.; Huang, Y. G.; Rogers, J. A. Stretchable ferroelectric nanoribbons with wavy configurations on elastomeric substrates. ACS Nano 2011, 5, 3326-3332.

[512] Lee, M. H.; Javey, A. Power surfing on waves. Nature 2011, 472, 304-305.

[513] Qi, Y.; Kim, J.; Nguyen, T. D.; Lisko, B.; Purohit, P. K.; McAlpine, M. C. Enhanced piezoelectricity and stretchability in energy harvesting devices fabricated from buckled PZT ribbons. Nano Lett. 2011, 11, 1331-1336.

[514] Xu, S. Y.; Shi, Y. Power generation from piezoelectric lead zirconate titanate nanotubes. J. Phys. D: Appl. Phys. 2009, 42, 085301.

[515] Xu, S.; Hansen, B. J.; Wang, Z. L. Piezoelectric-nanowireenabled power source for driving wireless microelectronics. Nat. Commun. 2010, 1, 93.

[516] Wang, Z. Y.; Hu, J.; Suryavanshi, A. P.; Yum, K.; Yu, M. F. Voltage generation from individual $\mathrm{BaTiO}_{3}$ nanowires under periodic tensile mechanical load. Nano Lett. 2007, 7, 2966-2969.

[517] Ke, T. Y.; Chen, H. A.; Sheu, H. S.; Yeh, J. W.; Lin, H. N.; Lee, C. Y.; Chiu, H. T. Sodium niobate nanowire and its piezoelectricity. J. Phys. Chem. C 2008, 112, 8827-8831.

[518] Chang, C.; Tran, V. H.; Wang, J.; Fuh, Y. K.; Lin, L. Direct-write piezoelectric polymeric nanogenerator with high energy conversion efficiency. Nano Lett. 2010, 10, 726-731.

[519] Hansen, B. J.; Liu, Y.; Yang, R. S.; Wang, Z. L. Hybrid nanogenerator for concurrently harvesting biomechanical and biochemical energy. ACS Nano 2010, 4, 3647-3652.

[520] Service, R. F. Nanogenerators tap waste energy to power ultrasmall electronics. Science 2010, 328, 304-305.

[521] Wang, Z. L. Piezoelectric nanostructures: From growth phenomena to electric nanogenerators. MRS Bull. 2007, 32, 109-116.

[522] Zhao, M. H.; Wang, Z. L.; Mao, S. X. Piezoelectric characterization of individual zinc oxide nanobelt probed by piezoresponse force microscope. Nano Lett. 2004, 4 , 587-590.

[523] Mitrushchenkov, A.; Linguerri, R.; Charnbaud, G. Piezoelectric properties of $\mathrm{AlN}, \mathrm{ZnO}$, and $\mathrm{Hg}_{x} \mathrm{Zn}_{1-x} \mathrm{O}$ nanowires by first-principles calculations. J. Phys. Chem. C 2009, 113, 6883-6886.

[524] Xiang, H. J.; Yang, J. L.; Hou, J. G.; Zhu, Q. S. Piezoelectricity in $\mathrm{ZnO}$ nanowires: A first-principles study. Appl. Phys. Lett. 2006, 89, 223111.

[525] Xin, J.; Zheng, Y.; Shi, E. Piezoelectricity of zinc-blende 
and wurtzite structure binary compounds. Appl. Phys. Lett. 2007, 91, 112902.

[526] grawal, R.; Espinosa, H. D. Giant piezoelectric size effects in zinc oxide and gallium nitride nanowires. A first principles investigation. Nano Lett. 2011, 11, 786-790.

[527] Gao, Y.; Wang, Z. L. Electrostatic potential in a bent piezoelectric nanowire. The fundamental theory of nanogenerator and nanopiezotronics. Nano Lett. 2007, 7, 2499 2505.

[528] Scrymgeour, D. A.; Hsu, J. W. P. Correlated piezoelectric and electrical properties in individual $\mathrm{ZnO}$ nanorods. Nano Lett. 2008, 8, 2204-2209.

[529] Gao, Y.; Wang, Z. L. Equilibrium potential of free charge carriers in a bent piezoelectric semiconductive nanowire. Nano Lett. 2009, 9, 1103-1110.

[530] Mora-Sero, I.; Fabregat-Santiago, F.; Denier, B.; Bisquert, J.; Tena-Zaera, R.; Elias, J.; Levy-Clement, C. Determination of carrier density of $\mathrm{ZnO}$ nanowires by electrochemical techniques. Appl. Phys. Lett. 2006, 89, 203117.

[531] Tong, H.; Wang, B. L.; Ou-Yang, Z. C. Electric potential generated in $\mathrm{ZnO}$ nanowire due to piezoelectric effect. Thin Solid Films 2008, 516, 2708-2710.

[532] Allen, M. W.; Alkaisi, M. M.; Durbin, S. M. Metal Schottky diodes on Zn-polar and O-polar bulk ZnO. Appl. Phys. Lett. 2006, 89, 103520.

[533] Coppa, B. J.; Davis, R. F.; Nemanich, R. J. Gold Schottky contacts on oxygen plasma-treated, $n$-type $\mathrm{ZnO}(000 \overline{1})$. Appl. Phys. Lett. 2003, 82, 400-402.

[534] Wenckstern, H. V.; Kaidashev, E. M.; Lorenz, M.; Hochmuth, H.; Biehne, G.; Lenzner, J.; Gottschalch, V.; Pickenhain, R.; Grundmann, M. Lateral homogeneity of Schottky contacts on $n$-type ZnO. Appl. Phys. Lett. 2004, 84, 79-81.

[535] Kim, S. H.; Kim, H. K.; Seong, T. Y. Effect of hydrogen peroxide treatment on the characteristics of Pt Schottky contact on $n$-type ZnO. Appl. Phys. Lett. 2005, 86, 112101.

[536] Rakhshani, A. E. Schottky diodes on $\mathrm{ZnO}$ rods grown homoepitaxially by successive chemical solution deposition. Semicond. Sci. Technol. 2008, 23, 075037.

[537] Periasamy, C.; Chakrabarti, P. Time-dependent degradation of $\mathrm{Pt} / \mathrm{ZnO}$ nanoneedle rectifying contact based piezoelectric nanogenerator. J. Appl. Phys. 2011, 109, 054306.

[538] Song, J. H.; Xie, H. Z.; Wu, W. Z.; Joseph, V. R.; Wu, C. F. J.; Wang, Z. L. Robust optimization of the output voltage of nanogenerators by statistical design of experiments. Nano Res. 2010, 3, 613-619.

[539] Shao, Z. Z.; Wen, L. Y.; Wu, D. M.; Zhang, X. A.; Chang, S. L.; Qin, S. Q. AFM analysis of piezoelectric nanogenerator based on $n^{+}$-diamond $/ n-Z n O$ heterojunction. Appl. Surf. Sci. 2011, 257, 4919-4922.
[540] Riaz, M.; Fulati, A.; Amin, G.; Alvi, N. H.; Nur, O.; Willander, M. Buckling and elastic stability of vertical $\mathrm{ZnO}$ nanotubes and nanorods. J. Appl. Phys. 2009, 106, 034309.

[541] Liu, J.; Fei, P.; Zhou, J.; Tummala, R.; Wang, Z. L. Toward high output-power nanogenerator. Appl. Phys. Lett. 2008, 92, 173105.

[542] Thundat, T. Flexible approach pays off. Nat.Nanotechnol. 2008, 3, 133-134.

[543] Zhang, J.; Li, M. K.; Yu, L. Y.; Liu, L. L.; Zhang, H.; Yang, Z. Synthesis and piezoelectric properties of well-aligned $\mathrm{ZnO}$ nanowire arrays via a simple solution-phase approach. Appl. Phys. A 2009, 97, 869-876.

[544] Park, H. K.; Lee, K. Y.; Seo, J. S.; Jeong, J. A.; Kim, H. K.; Choi, D.; Kim, S. W. Charge-generating mode control in high-performance transparent flexible piezoelectric nanogenerators. Adv. Funct. Mater. 2011, 21, 1187-1193.

[545] Choi, D.; Choi, M. Y.; Shin, H. J.; Yoon, S. M.; Seo, J. S.; Choi, J. Y.; Lee, S. Y.; Kim, J. M.; Kim, S. W. Nanoscale networked single-walled carbon-nanotube electrodes for transparent flexible nanogenerators. J. Phys. Chem. C 2010, 114, 1379-1384.

[546] Choi, D.; Choi, M. Y.; Choi, W. M.; Shin, H. J.; Park, H. K.; Seo, J. S.; Park, J.; Yoon, S. M.; Chae, S. J.; Lee, Y. H.; Kim, S. W.; Choi, J. Y.; Lee, S. Y.; Kim, J. M. Fully rollable transparent nanogenerators based on graphene electrodes. Adv. Mater. 2010, 22, 2187-2192.

[547] Shin, H. J.; Choi, W. M.; Choi, D.; Han, G. H.; Yoon, S. M.; Park, H. K.; Kim, S. W.; Jin, Y. W.; Lee, S. Y.; Kim, J. M.; Choi, J. Y.; Lee, Y. H. Control of electronic structure of graphene by various sopants and their effects on a nanogenerator. J. Am. Chem. Soc. 2010, 132, 15603-15609.

[548] Kumar, B.; Lee, K. Y.; Park, H. K.; Chae, S. J.; Lee, Y. H.; $\mathrm{Kim}, \mathrm{S}$. W. Controlled growth of semiconducting nanowire, nanowall, and hybrid nanostructures on graphene for piezoelectric nanogenerators. ACS Nano 2011, 5, 41974204.

[549] Yang, R. S.; Qin, Y.; Li, C.; Dai, L. M.; Wang, Z. L. Characteristics of output voltage and current of integrated nanogenerators. Appl. Phys. Lett. 2009, 94, 022905.

[550] Yang, R.; Qin, Y.; Li, C.; Zhu, G.; Wang, Z. L. Converting biomechanical energy into electricity by a muscle-movementdriven nanogenerator. Nano Lett. 2009, 9, 1201-1205.

[551] Li, Z.; Zhu, G.; Yang, R. S.; Wang, A. C.; Wang, Z. L. Muscle-driven in vivo nanogenerator. Adv. Mater. 2010, 22, 2534-2537.

[552] Zhu, G.; Yang, R. S.; Wang, S. H.; Wang, Z. L. Flexible high-output nanogenerator based on lateral $\mathrm{ZnO}$ nanowire array. Nano Lett. 2010, 10, 3151-3155. 
[553] Agrawal, R.; Peng, B.; Espinosa, H. D. Experimentalcomputational investigation of $\mathrm{ZnO}$ nanowires strength and fracture. Nano Lett. 2009, 9, 4177-4183.

[554] Yu, A. F.; Li, H. Y.; Tang, H. Y.; Liu, T. J.; Jiang, P.; Wang, Z. L. Vertically integrated nanogenerator based on $\mathrm{ZnO}$ nanowire arrays. Phys. Status Solidi R 2011, 5, 162-164.

[555] van der Heyden, F. H. J.; Bonthuis, D. J.; Stein, D.; Meyer, C.; Dekker, C. Power generation by pressure-driven transport of ions in nanofluidic channels. Nano Lett. 2007, 7, 10221025.

[556] Hu, Y.; Zhang, Y.; Xu, C.; Lin, L.; Snyder, R. L.; Wang, Z. L. Self-powered system with wireless data transmission. Nano Lett. 2011, 11, 2572-2577.

[557] Khan, S. U. M.; Al-Shahry, M.; Ingler, W. B. Efficient photochemical water splitting by a chemically modified n- $\mathrm{TiO}_{2}$ Science 2002, 297, 2243-2245.
[558] Chen, H.; Chen, C.; Chang, Y. C.; Tsai, C. W.; Liu, R. S.; Hu, S. F.; Chang, W. S.; Chen, K. H. Quantum dot monolayer sensitized $\mathrm{ZnO}$ nanowire-array photoelectrodes: True efficiency for water splitting. Angew. Chem. Int. Ed. 2010, 49, 5966-5969.

[559] Wang, G. M.; Yang, X. Y.; Qian, F.; Zhang, J. Z.; Li, Y. Double-sided CdS and CdSe quantum dot co-sensitized $\mathrm{ZnO}$ nanowire arrays for photoelectrochemical hydrogen generation. Nano Lett. 2010, 10, 1088-1092.

[560] Wang, X. W.; Liu, G.; Lu, G. Q.; Cheng, H. M. Stable photocatalytic hydrogen evolution from water over $\mathrm{ZnO}-\mathrm{CdS}$ core-shell nanorods. Int. J. Hydrogen Energy 2010, 35, 8199-8205.

[561] Hong, K. S.; Xu, H. F.; Konishi, H.; Li, X. C. Direct water splitting through vibrating piezoelectric microfibers in water. J. Phys. Chem. Lett. 2010, 1, 997-1002. 